\title{
Proceedings of the 11th international congress of the metastasis research society
}

\section{Keynote Lecture 1}

\section{Cancer genomics: from bench to bedside}

Yusuke Nakamura

Human Genome Center, Institute of Medical Science, The University of Tokyo, Japan

cDNA microarray technologies have enabled us to obtain comprehensive data for gene expression profiles of human cancers. To isolate novel targets for diagnosis (tumor marker or predictive marker for the efficacy of treatment) as well as treatment of cancer (moleculartargeting drug, cancer vaccine, siRNA, and antibody), we have been comparing expression profiles of cancer cells originated from various organs with their corresponding noncancerous tissues using a cDNA microarray that consists of nearly 32,000 genes. These experiments disclosed a number of genes that appeared to be involved in development and/or progression of cancers in those tissues. We have selected hundreds of candidate genes by the criteria as follows; (1) gene expressions were transactivated in a large proportion of cancer tissues in comparison with their corresponding normal tissues and (2) expression was not observed or hardly detectable in any of important vital organs. The further functional analysis identified dozens of genes that are likely to function as oncogenes in various cancers. The suppression of expression of such genes with small-interfering RNAs (siRNAs) induced cell cycle arrest, apoptosis, or suppression of anchoringdependent cell growth.

For example, one of the good candidates that we identified as a molecular target for development of drugs was SMYD3, a gene that is over-expressed in the majority of colorectal carcinomas, hepatocellular carcinomas and breast cancers. Introduction of SMYD3 into NIH3T3 cells enhanced cell growth, whereas genetic knockdown with siRNA in cancer cells resulted in significant growth suppression. SMYD3 formed a complex with RNA polymerase II through an interaction with the RNA helicase HELZ and transactivated a set of genes that included oncogenes, homeobox genes and genes associated with cell-cycle regulation. The SET domain of SMYD3 showed histone H3lysine 4 (H3K4)-specific methyltransferase activity, which was enhanced in the presence of the heat-shock protein HSP90A. Our findings suggest that SMYD3 has histone methyltransferase activity and plays an important role in transcriptional regulation as a member of an RNA polymerase complex.

In addition, we have been developing antibodies to these candidate molecules and found some antibodies that showed growth-suppressive effect in vivo and/or in vitro. As a good example, we recently reported that Frizzled homologue 10 (FZD10), a member of Frizzled family, is exclusively up-regulated in synovial sarcoma (SS) and its expression is not or hardly detectable in normal organs except placenta. We subsequently established two murine monoclonal antibodies (92-13 and 93-22 Mabs) to FZD10 by immunizing mice with FZD10-transfected cells. Both 92-13 and 93-22 Mabs were shown to have specific binding activity against FZD10 on cell lines expressing FZD10 by flow cytometry (FACS) analysis. To further validate the specific binding activity of those antibodies in vivo, we injected fluorescent-labeled Mabs intraperitoneally or intravenously into the mice carrying SS xenografts and found 
that these Mabs were bound to the FZD10-expressing tumors, but not to any other normal mouse tissues by the use of the in vivo fluorescent imaging system. Immunohistochemical analyses with these Mabs confirmed an absence or hardly-detectable level of FZD10 protein in normal human organs except the placenta. Furthermore, we found that the Mabs were internalized into the SS cell line, SYO-1, but not into FZD10-negative cell line, LoVo using confocal laser scanning microscopy. Taken together, these specific Mabs against FZD10 could be utilized as the novel diagnostic marker or treatment of SS with minimal or no risk of adverse reactions. Our results indicated that systematic expression analysis should be a very effective approach for identification of molecules that are potential targets for development of novel therapeutic drugs and diagnostic tools.

\section{Keynote Lecture 2}

\section{TGF-beta: from transforming growth factor to tumor suppressor}

\section{Harold L. Moses}

Vanderbilt-Ingram Cancer Center and Department of Cancer Biology, Vanderbilt University Medical Center, Nashville, TN, USA

Although originally described as an activity that caused nontransformed cells to grow in an anchorage independent, there is now compelling evidence from transgenic mouse studies and analysis of mutations in human carcinomas indicating that the TGF-beta signal transduction pathway is tumor suppressive. A number of transgenic mouse studies demonstrated that overexpresssion of TGF-betal in epithelial cells suppressed tumor formation while expression of a dominant negative type II TGF-beta receptor enhanced carcinoma development. Studies of human tumors have demonstrated inactivating mutations in human tumors of genes encoding proteins involved in TGF-beta signal transduction, including DPC4/Smad4, Smad 2, and the type I and type II TGF-beta receptor (TBRI and TBRII, respectively).

There is also evidence that TGF-beta can enhance the progression of tumors. This hypothesis has also tested in genetically modified mice. To attain complete loss of TBRII, we have generated mice with loxP sites flanking exon 2 of $T g f b r 2$ and crossed them with mice expressing Cre recombinase under control of the MMTV promoter/enhancer to obtain Tgfbr $2^{\mathrm{mgKO}}$ mice.
These mice show mammary lobuloalveolar hyperplasia. Tgfbr $2^{\mathrm{mgKO}}$ mice that also express polyoma virus middle $\mathrm{T}$ antigen under control of the MMTV promoter (MMTV-PyVmT) develop mammary tumors with a significantly shorter latency than MMTV-PyVmT mice and show a marked increase in pulmonary metastases. Our data support the hypothesis that TGF-beta signaling in mammary carcinoma cells can also have tumor suppressive actions even after carcinomas are well established.

The importance of stromal-epithelial interactions in mammary gland development and tumorigenesis is well established. These interactions likely involve autocrine and paracrine action of multiple growth factors, including members of the TGF-beta family, which are expressed in both stroma and epithelium. Again, to accomplish complete knockout of the type II TGF-beta receptor gene in mammary stromal cells FSP1-Cre and Tgfbr $2^{\text {flox/flox }}$ mice were crossed to attain Tgfbr $2^{\text {fspKO }}$ mice. The loss of TGF-beta responsiveness in fibroblasts resulted in intraepithelial neoplasia in prostate and invasive squamous cell carcinoma of the forestomach with high penetrance by six weeks of age. Both epithelial lesions were associated with an increased abundance of stromal cells. Activation of paracrine hepatocyte growth factor (HGF) signaling was identified as one mechanism for stimulation of epithelial proliferation. Tgfbr $2^{\text {fspKO }}$ mice also exhibit defective mammary ductal development, characterized in part by increased ductal epithelial cell turnover associated with an increase in stromal fibroblast abundance. Tgfbr $2^{\text {fspKO }}$ mammary fibroblasts transplanted with mammary carcinoma cells promote growth and invasion, which is associated with increased activating phosphorylation of the receptors: erbB1, erbB2, RON, and c-Met. Furthermore, the increased receptor phosphorylation correlates with increased secretion of the cognate ligands by $\mathrm{Tgfbr} 2^{\text {fspKO }}$ fibroblasts. Treatment of tumor cells with fibroblast conditioned medium leads to increased tumor cell proliferation and motility, which are blocked by addition of pharmacologic inhibitors of TGF-alpha signaling or neutralizing antibodies to MSP, HGF, or c-Met. A small molecule inhibitor of Met signaling inhibited tumor growth, invasion and metastasis in vivo. These studies demonstrate a significant role for stromal TGFbeta signaling in mammary tissue homeostasis and mammary tumor progression via regulation of TGFalpha, MSP, and HGF signaling pathways.

Thus, TGF-beta signaling in fibroblasts modulates the growth and oncogenic potential of adjacent epithelia in selected tissues. The Tgfbr $2^{\text {fspKO }}$ mouse model illustrates that a signaling pathway known to suppress 
cell-cycle progression when activated in epithelial cells can also have an indirect inhibitory effect on epithelial proliferation when activated in adjacent stromal fibroblasts in vivo. Loss of this inhibitory effect can result in increased epithelial proliferation and may even progress to invasive carcinoma in some tissues.

\section{Presidential Lecture}

\section{Critical determinants of organ specific lung cancer metastasis for therapy}

\section{Saburo Sone}

Department of Internal Medicine and Molecular Therapeutics Institute of Health Biosciences, The University of Tokushima Graduate School, Japan

The development of cancer metastasis depends on multiple interactions (cross-talk) between selected metastatic tumor cells ("seed") and the microenvironment of specific organs ("soil"). Clinically, lung cancer on diagnosis is frequently associated with the metastases to multiple organs such as liver, lung, lymph nodes, brain, and bone. Little is known, however, of what genes and/ or molecules in lung cancer mediate the organ-site specific metastatic tropism under particular organ microenvironments. To address this question, we developed novel in vivo multiple organ metastasis models of human lung cancer in NK cell-depleted SCID mice. Cells with or without gene modification were injected into a tail vein to examine their metastatic patterns. For example, overexpression of CD 82 or IL- $1 \beta$ increased the metastatic potential to multiple organs. We also found that the metastasis of MMP nonproducing lung cancer to liver, but not to kidney or lymph node depended on MMP expressed in host liver tissue. On the other hand, gene-modification of lung cancer cells with $\mathrm{M}-\mathrm{CSF}$ resulted in marked inhibition of metastasis to liver and lymph node, but not to kidney, because of much fewer macrophages in the kidney. We also observed that Reveromycin targeting leucyl t-RNA synthetase caused apoptosis of osteoclast, which was associated with inhibition of bone metastasis, but no inhibitory effects on metastases to other organs such as lung, liver and kidney. These results clearly suggest that metastatic development of lung cancer might be influenced by the interaction between cancer cells and host factors under the organ-site specific microenvironments.

In series of this study, we performed cDNA-microarray analyses (23,040 genes) of tumor samples obtained by microdissection from the metastatic foci of the four different organs (bone, lung, liver and kidney) in order to elucidate the critical genes regulating organ-site specific metastasis. By comparing the gene-expression profiles of metastatic lesions classified according to size $(<1$ or $>2 \mathrm{~mm})$ as either "micrometastases" or "macrometastases." we found that many of the 105 genes involved in the incipient stage of secondary-tumor formation were responsible for cell motility, cell adhesion and ECM remodeling. Interestingly, three chemokine receptors (CCR4, CCR5, CCR9) were equally expressed in all the lesions. On the other hand, hierarchical clustering of 435 genes separated the four organspecific groups of macrometastatic lesions very clearly. For example, PTHrP was highly expressed in osteolytic bone metastasis. Inhibition of PTHrP by neutralizing antibody resulted in specific inhibition of bone metastasis associating decreased number of osteoclasts in the bone lesions, indicating that PTHrP plays a critical role on the production of osteolytic bone metastasis. Follistatin (FST), activin-binding protein, was also up-regulated only in the bone metastasis. Interestingly, transfection of FST gene into SBC-5 cells resulted in inhibition of metastasis not only to the bone but also to the visceral organs. In addition, the number of proliferating tumor cells and tumor microvessel density were significantly reduced in the lesions produced by FSTtransfectants. Since the transfection did not affect the characteristics of SBC-5 cells in vitro, FST may modify the interaction between tumor cells and host microenvironments.

In summary, we have developed a novel, in vivo multiple organ metastatic models of human lung cancer and used it to identify the critical determinants of general and organ-specific metastasis of lung cancer under the organ microenvironments. This approach leads to the development of the molecular-target therapy for organ-specific metastasis of lung cancer.

\section{JAMR Special Award Lecture}

\section{The organ microenvironment in metastasis: biology and therapy}

Isaiah J. Fidler

Department of Cancer Biology, The University of Texas MD Anderson Cancer Center, USA

The major cause of death from cancer is metastases that are resistant to conventional therapies. To produce a metastasis, tumor cells must complete a series of sequential and highly selective steps whose outcome is determined by the interactions of tumor cells with homeostatic host mechanisms. Preferential metastasis of tumor cells to certain organs is independent of vascular anatomy, rate of blood flow, and number of tumor cells 
delivered to each organ. The outcome of metastasis depends on multiple continuous interactions between unique subpopulations of tumor cells ("seed") and specific host factors within the organ microenvironment, such as vasculature ("soil").

Understanding the mechanisms responsible for the development of biological heterogeneity in primary cancers and metastases and the processes that regulate tumor cell dissemination to and proliferation in distant tissues is a major goal of research. For many years, all of our efforts to treat cancer metastases have concentrated on the inhibition or destruction of tumor cells. New strategies to treat tumor cells by modulating their interaction with the organ microenvironment and by targeting tumor-associated vasculature present unprecedented possibilities for the treatment of cancer metastasis.

\section{Paget-Ewing Award Lectures}

\section{Matrix metalloproteinases in cancer metastasis}

\section{Lynn M. Matrisian}

\section{Vanderbilt University Medical Center}

As recognized by Sir Stephen Paget in 1889, the tumor microenvironment has a profound effect on the establishment of metastatic lesions, providing a "congenial soil" for specific tumor "seeds". There is considerable focus on identifying the molecules responsible for mediating the communication between tumor cells and the surrounding stroma to identify novel targets for anti-metastatic therapy. The matrix metalloproteinases (MMPs), long regarded as key enzymes involved in matrix destruction associated with tumor invasion, are also now recognized to be "sheddases" responsible for the activation, inactivation, and solubilization of biologically active factors that regulate processes such as tumor cell survival, growth, and angiogenesis. Although clinical trials with synthetic MMP inhibitors failed to demonstrate a therapeutic benefit, there is abundant preclinical data demonstrating that inhibition of specific MMPs at specific stages of tumor progression is efficacious in slowing tumor progression, reducing tumor burden, or minimizing metastasis. The issue is determining how to best apply this knowledge for therapeutic benefit.

MMP-null mice combined with transplantable tumor models provides the opportunity to assess the contribution of specific host-derived MMP family members to tumor progression. Using orthotopic implantation of human NSCLC cells in the lungs of immunocompromised mice, the expression of stromal (murine) and tumor (human) protease mRNA was assessed using a custom Affymetrics $\mathrm{Hu} / \mathrm{Mu}$ ProtIn array. MMP12, MMP13, and cathepsin $\mathrm{K}$ were identified as stromal enzymes induced by the tumor, and their elevated expression in human tumors was confirmed by mining of array data from human NSCLC specimens. MMP12, also known as macrophage metalloelastase, was expressed primarily by cells resembling macrophages. Murine LLC lung carcinoma cells were injected into the tail vein of wildtype and MMP12-null mice. There was no effect on the overall establishment of metastatic lesions in this experimental metastasis assay, but the number of large tumors ( $>2 \mathrm{~mm}$ diameter) was significantly increased in MMP12-null mice compared to wildtype mice. This effect correlated with a significant increase in CD31-positive cells representing new vasculature, and with a significant decrease in the circulating levels of the K1-K4 form of angiostatin, a potent antiangiogenic peptide derived by cleavage of angiostatin by several MMPs, including MMP12. These results indicate that, in contrast to the assumption that MMPs promote tumor progression, stromal MMP12 has a protective role in the angiogenic switch by generating the antiangiogenic molecule angiostatin.

Other stromal MMPs promote the formation of metastatic lesions. When mice deficient in MMP9 were challenged in experimental metastasis assays examining metastasis to the lung or the liver, there was a significant reduction in the establishment of metastatic lesions. Interestingly, the source of the MMP9 was bone marrow-derived cells in the case of lung metastasis, and resident cells in the case of liver metastasis, demonstrating organ-specific differences in the response. In addition, the response was specific for individual MMP family members, as neither MMP2 nor MMP7 deficiency reduced the establishment of lung or liver metastases in these model systems.

We have also demonstrated that several stromal MMPs contribute to the "vicious cycle" that influences the growth of breast and prostate cancer cells in the bone microenvironment. Mice deficient in MMP2 and MMP9 demonstrate a reduction in the growth of murine mammary carcinoma cells injected into the tibia compared to wildtype control mice. In addition, mice deficient in MMP7 demonstrate reduced growth and osteolysis of prostate cancer cells transplanted on the skull. This effect was mediated by a release of soluble RANKL, a TNF-alpha family member that stimulates the activation of osteoclasts. 
These results suggest that stromal MMPs can be effective targets for anti-metastatic agents, but the specific "seed", the specific "soil", and the specific MMP family member must be identified to effectively translate these findings for clinical benefit.

\section{Educational Lecture 1}

\section{Superoxide and metastasis}

Y. Niitsu, K. Kuribayashi, K. Nakamura, M. Tanaka, Araki, T. Sato, R. Takimoto, T. Matsunaga,

T. Takayama, J. Kato

Fourth Department of Internal Medicine, Sapporo Medical University School of Medicine, Japan

Metastasis of tumor cells consists of multiple steps, including neovascularization, detachment from primary site, invasion, passive transportation by circulation and lodging and growth at metastatic foci. Molecular mechanisms underlying each step have been recently well elucidated: overexpression of neovascularization factors in tumor cells, impaired expression of cell-cell adhesion molecules of tumor cells, increased metalloprotease activity and decreased expression of TIMP by tumor cells, etc.

Over the past decade, we have focused our interest on interaction of interstitial cells with tumor cell metastasis, particularly on the activity of reactive oxygen radicals (ROS), which may be generated from inflammatory cells that have infiltrated the tumor and facilitate tumor cell motility.

Using a gold colloidal phagokinetic track assay and a boyden chamber, we demonstrated that superoxide generated by a xanthine/xanthine oxidase reaction stimulated tumor cell (SASH-1) motility, which was significantly inhibited by addition of $N$-acetyl-L-cysteine (NAC) or transduction of the superoxide dismutase $(S O D)$ gene. The SOD transfectants showed impaired metastatic potential compared to their parental cells in both artificial and spontaneous metastasis models. We then found that the cell motility signal induced by superoxide stimuli was transduced by the primary target, PKC, which phosphorylated the rhoGDI/small G protein complex. Upon phosphorylation of the complex, small G proteins were released from rhoGDI and activated to form stress fibers, lamelipodia and filopodia, leading to increased cell motility.

When each of these steps of the signal pathway were blocked by their specific inhibitors, the cell motility was clearly reduced even in the presence of superoxide stimuli. These results indicate that each step of this signal pathway could be a candidate target for antimetastatic drugs.
In particular, $\mathrm{PKC} \zeta$ may serve as the most viable target because of its relative specificity to superoxide stimulation.

In this lecture, I will review the relevance of ROS to metastasis, presenting our own results and discuss the feasibility of targeting $\mathrm{PKC} \zeta$ as a novel antimetastatic approach.

\section{Educational Lecture 3}

\section{Current challenges in cancer research}

Margaret L. Kripke

The University of Texas MD Anderson Cancer Center, USA

Cancer as a disease poses many challenges at the present time. Some are universal, and some are unique to the United States. The major challenges to managing cancer in the U.S. today include the following issues: (1) Increasing numbers of cancer patients, owing to aging of the population, (2) Heterogeneity in the treatments received in different locations and by different populations, (3) The growing population of cancer survivors, and (4) Limited resources

There are also many challenges associated with translating advances in the laboratory into practical approaches to cancer treatment. The President's Cancer Panel, which advises the President of the United States regarding the nation's cancer program, reviewed these challenges in their 2004/2005 report entitled, "Translating Research into Cancer Care: Delivering on the Promise". Translational research represents a continuum from basic science discovery to applying research findings to the disease of human cancer, formulating and conducting research on cancer patients, and finally, disseminating the results to the oncology community. Barriers to progress are present at each step in the process. Findings and recommendations from this report will be reviewed, and a status report of progress made on several of the recommendations will be presented.

\section{Educational Lecture 4}

\section{Alteration of E-cadherin-mediated cell adhesion in cancer metastasis}

Setsuo Hirohashi

National Cancer Center Research Institute, Japan

Metastasis is a complex process playing a key role in cancer progression. Pathological studies have revealed that histological features such as stromal invasion, 
lymphatic/venous invasion and lymph node metastasis are hallmarks of metastatic cancer, and cancer cell dissociation is the primary and essential event that precedes these phenomena. E-cadherin is an epitheliumspecific cell-cell adhesion molecule that plays an essential role in maintaining epithelial structural integrity. We and others have reported that E-cadherin-mediated cell adhesion is inactivated by multiple molecular mechanisms (genetic and epigenetic alterations) in cancer and is significantly implicated in metastasis.

Recently we have reported that a novel glycoprotein, dysadherin, downregulates E-cadherin protein expression in a post-transcriptional manner and induces cancer cell migration. Dysadherin is overexpressed in many cancers and its expression is significantly associated with the malignant potential of tumors. Forced expression of dysadherin in pancreatic cancer cell lines significantly promotes metastasis in vivo, whereas reduction of dysadherin expression by specific short interfering RNA affects the organization of the actin cytoskeleton and inhibits cancer cell migration.

Loss of intercellular adhesion also promotes cancer cell migration through downstream signal pathways. Lobular carcinoma of the breast harbors E-cadherin mutations and frequently shows distant metastasis clinically. In this tumor, loss of E-cadherin promotes dislocation of p120ctn, one of the proteins associated with E-cadherin, from the plasma membrane to the cytoplasm. Cytoplasmic p120ctn modulates the activities of small G-proteins such as Rho and stimulates cancer cell migration. $\beta$-Catenin, another E-cadherinassociated molecule, is stabilized by mutations of the $A P C$ gene in most colon cancers. We found prominent accumulation of $\beta$-catenin protein in the migrative membrane of colon cancer cells that had lost their E-cadherin expression. By application of highly sensitive mass-spectrometry, we identified actinin-4, an actin-binding protein, in association with $\beta$-catenin, suggesting that this molecular complex may stimulate cell migration in the invasive front of colon cancer.

An understanding of the molecular pathways operating in cancer metastasis should provide clues to ways in which it can be inhibited or predicted. It has been shown that restoration of E-cadherin expression in E-cadherin negative breast cancer cell lines prevents lung metastasis in an experimental model, supporting the concept that both the E-cadherin complex and its downstream signal pathways, alterations of which potentiate some of the characteristics of metastasis (such as loss of cell adhesion and increased cell migration), could be promising therapeutic targets for slowing or preventing cancer metastasis. The combined expression of several metastasis-related molecules in colon cancer has been shown to be useful for predicting the likelihood of metastasis to the liver.

Inactivation of intercellular adhesion has long been considered to play essential roles in cancer invasion and metastasis, but our current knowledge of the molecular mechanisms connecting these pathobiological processes, especially in the context of tissue or cell-type specificity, is still incomplete. A broader understanding of these molecular processes will provide clinically useful targets for inhibition, treatment and prevention of cancer metastasis in future.

\section{Presidential Symposium 1}

\section{Issues and challenges in the development of biologically-targeted therapeutics for cancer-understanding targets, diseases, mechanisms and outcomes}

\section{Alan J. Barge}

\section{AstraZeneca, UK}

Many of the biological processes involved in the proliferation, dissemination and survival of tumour cells are characterised at a molecular level. Many genotypic and phenotypic abnormalities have been identified, and many of the molecular events leading to oncogenesis, proliferation, angiogenesis and invasion described. However, despite the enormous advance in descriptive science, the functional significance of many of the abnormalities remains uncertain. Many defects identified in cancer cells probably represent the molecular archaeology of the cancer they contributed to the generation of the malignant phenotype, but they may be redundant in driving the growth or spread of the tumour now.

This presents many challenges for the development of molecular targeted therapies. Understanding the biological role of specific abnormalities in individual tumours is critical to determining the potential benefits of inhibitors. Few human malignancies result from single genetic abnormalities. Where such single abnormalities have been demonstrated, such as in CML, dramatic responses to targeted therapy with imatinib have resulted.

The dramatic improvements in the technology for synthesising and selecting small molecule enzyme inhibitors, and for the generation of highly specific fully-human monoclonal antibodies have lead to an abundance of compounds available for clinical evaluation. The challenge has moved from molecule generation to disease-linkage, where the true biological relevance of the target is inherently more of an issue than making a drug to target it. 
The use of targeted agents in unselected populations of patients has illustrated the complexities of the biology of common cancers. Gefitinib (Iressa ${ }^{\circledR}$ ) are small molecule inhibitors of the tyrosine kinase R), and erlotinib (Tarceva ${ }^{\circledR}$ of the EGF receptor and have shown dramatic activity in some populations of patients with NSCLC. Extensive profiling of tissue from patients in large randomised studies of both drugs has defined a molecular profile predictive of benefit. However, this has required large studies in unselected populations initially to demonstrate this differential effect, and to allow robust confirmation of the initial hypothesis. AS further targeted agents enter the clinic, similar challenges will follow.

\section{Presidential Symposium 2}

\section{Matuzumab: a humanized EGFR-targeted monoclonal antibody}

\section{Philip Breitfeld}

\section{EMD Pharmaceuticals, USA}

Binding of natural ligands to EGFR triggers intracellular signals that regulate cancer cell growth. Antibodies to EGFR can block these signals and forms the basis for the hypothesis that EGFR is a target in cancer therapy. This hypothesis achieved clinical validation with marketing approval of anticancer drugs that block EGFR signaling.

Inhibition of natural ligand-induced EGFR signaling has the potential to inhibit cancer cell metastasis. For example, EGF promotes tumor cell migration by inducing ADAM10-mediated CD44 cleavage through Rac1 and mitogen-activated protein kinase activation. Overexpression of EGFR in MTLn3 mammary adenocarcinoma cells results in increased lung metastasis from tumors formed by injection of cells in the mammary fat pad.

The proposed mechanisms of action for EGFR-targeted monoclonal antibodies are (1) inhibition of native ligand binding to receptor, thus blocking the mitogenic intracellular signaling through the Ras-Raf-MAPK, and PI-3 kinase/Akt transduction pathways, (2) downregulation of EGFR expression by removing the receptor from the tumor cell surface, (3) inhibition of receptor dimerization, and (4) stimulation of the immunological response termed antibody-dependent cell-mediated cytotoxicity (ADCC) which occurs only with the IgG1 subtype monoclonal antibodies.

Matuzumab (EMD 72000) is a humanized IgG1 antibody specific for the EGFR. Matuzumab induced natural killer cell-mediated ADCC against tumor cells in vitro, and de-glycosylated matuzumab (ablating ADCC) has diminished anti-tumor activity (but similar
PK characteristics) suggesting in an in vivo animal model, ADCC is a potential mechanism of action.

Using in vivo tumor models, Matuzumab monotherapy has demonstrated activity against human lung and gastric adenocarcinomas, and chemotherapy-resistance pancreatic carcinomas. The combination of matuzumab and gemcitabine was more effective than either agent alone in inhibiting growth of human pancreatic cancer xenografts.

Matuzumab pharmacokinetic studies have found (1) first-order kinetics at clinically utilized doses, (2) a long elimination half-life, ranging from 6 to 10.5 days depending on dose and schedule, (3) dose-proportional increases in maximum plasma concentration and area under the time-concentration curve, and (4) trough level at clinically utilized doses well above that required for complete in vitro tumor growth inhibition. Pharmacokinetic findings in Japanese patients are similar. The maximum tolerated dose of Matuzumab is $1,600 \mathrm{mg} \mathrm{QW}$ with dose-limiting toxicity being grade III headache and fever. A phase I trial in 26 Japanese patients confirmed the safety of matuzumab at doses up to $1,600 \mathrm{mg}$ QW.

In monotherapy trials, the most common drug-related adverse event has been skin toxicity, consisting of a grade 1 or 2 acneiform rash or epidermolysis occurring in approximately $67 \%$ of patients. Grade 3 or 4 skin toxicity has been $<1 \%$. Other grade 1 and 2 toxicities were headache, fever, and diarrhea (in approximately $15-30 \%$ of patients).

In a phase I study, tumor growth control was achieved in $50 \%$ of patients (5 PRs: 2 with colorectal cancer, 2 with head and neck cancer, and 1 with esophageal cancer; 6 SD). In 26 Japanese patients with previously treated EGFR-positive advanced solid tumors, tumor growth control (2 PR $+10 \mathrm{SD})$ was achieved by $46 \%$ of patients.

In advanced pancreatic adenocarcinoma, gemcitabine plus escalating doses of matuzumab demonstrated a $47 \%$ disease control rate (3 PRs and 5 SD). In a phase I study in 1 st line advanced gastric cancer using escalating doses of matuzumab in combination with epirubicin, cisplatin, and capecitabine, disease control rate was $81 \%$ (7 PRs, and 10 had SD). Early data from a phase I trial of matuzumab in combination with cisplatin, 5-fluorouracil, and leucovorin in advanced gastric cancer demonstrated a disease control rate of $78 \%$ (6 PRs, $1 \mathrm{SD})$. In a phase I trial of paclitaxel $\left(175 \mathrm{mg} / \mathrm{m}^{2}\right)$ in combination with escalating doses of matuzumab $(100,200,400$, and $800 \mathrm{mg}$ given QW) in NSCLC, the disease control rate was $55 \%$ (4 PR, $6 \mathrm{SD})$.

There are ongoing phase II studies in 2 nd line colorectal cancer, 1 st line metastatic gastric cancer, and 2nd line advanced NSCLC. 


\section{Presidential Symposium 3}

\section{Angiogenesis and lymphangiogenesis: from genetic insights to therapeutic strategies}

\section{Peter Carmeliet}

Adjunct Director, Department of Transgene Technology and Gene Therapy VIB, the Flanders Interuniversity Institute for Biotechnology Campus Gasthuisberg, Belgium

The growth of blood vessels - angiogenesis - is essential for organ growth and repair. An imbalance in this process contributes to numerous malignant, inflammatory, ischaemic, infectious and immune disorders.

Numerous genetic and pharmacological studies have revealed VEGF as a key player of the angiogenic switch in normal health but also in disease. Although antiVEGF antibody has been approved for treatment of colon cancer, responses are often short-lived, and resistance frequently emerges. We are therefore executing studies on the use of antibodies against PlGF as an anti-angiogenic therapy for cancer, which may provide a suitable and safe alternative or adjunct to VEGF blockade.

VEGF has also been implicated in other disorders such as neurodegeneration, respiratory distress, etc. and ongoing studies reveal that VEGF can be used to treat the paralyzing motoneuron degeneration disorder ALS.

A most exciting novel research avenue is the recent realization that vessels often use similar guidance signals as axons to navigate to their target. Understanding the molecular basis of vessel pathfinding offers unprecedented scientific opportunities to further unravel the molecular basis of angiogenesis and, likely, may provide valuable therapeutic and valorizable insights to promote or inhibit angiogenesis. The zebrafish model is particularly suited to address these issues, but we are also using Xenopus tadpoles and mice to complement these studies.

These genetic insights and their therapeutic potentials will be discussed.

\section{Presidential Symposium 4}

\section{Scientific rationale and clinical development of sunitinib malate}

Eiji Ueda ${ }^{1}$, Paolo G. Casali ${ }^{2}$, George D. Demetri ${ }^{3}$, Gary R. Hudes ${ }^{4}$, Matthew H. Kulke ${ }^{3}$, Kathy D. Miller ${ }^{5}$,

Robert J. Motzer ${ }^{6}$, Charles M. Baum ${ }^{7}$

${ }^{1}$ Pfizer Japan Inc., Japan, ${ }^{2}$ Istituto Nazionale Tumori, Italy, ${ }^{3}$ Dana-Farber Cancer Institute, USA, ${ }^{4}$ Fox Chase
Cancer Center, USA, ${ }^{5}$ Indiana University Cancer Center, USA, ${ }^{6}$ Memorial Sloan-Kettering Cancer Center, USA, ${ }^{7}$ Pfizer Inc., USA

Sunitinib malate (SU11248; SUTENT ${ }^{\circledR}$ ) is an oral, multitargeted tyrosine kinase inhibitor that selectively and potently inhibits the class III and V split kinase domain receptor tyrosine kinases (RTKs) VEGFR-1, 2 and -3 , PDGFR $-\alpha$ and $-\beta$, KIT, RET, CSF-1R, and FLT3. Per early characterization in biochemical assays, sunitinib was studied in cell lines that were engineered to, or normally, express VEGFR-2, PDGFR- $\beta$, KIT, FLT3-ITD, or a mutant form of RET associated with endocrine tumors; autophosphorylation of each was inhibited at a comparable potency range (0.008$0.05 \mu \mathrm{M})$. Subsequent studies of several rodent tumor models demonstrated time- and dose-dependent inhibition of critical RTK targets (i.e., VEGFR-2 and PDGFR- $\beta$ ), and dose-dependent antitumor efficacy. Based on these favorable preclinical findings, clinical studies in healthy volunteers began in December 2000 and in cancer patients in April 2001. Sunitinib has since demonstrated efficacy in two phase II trials of metastatic renal cell carcinoma (mRCC), and in phase I/II and III trials of patients with gastrointestinal stromal tumor (GIST) following failure of imatinib therapy due to resistance or intolerance. In the two mRCC trials, objective response rates (ORRs) of $>40 \%$ were observed with up to $27 \%$ of patients exhibiting prolonged stable disease (SD). In the phase II study of GIST, sunitinib treatment resulted in an ORR of $8 \%$ and a durable ( $\geq 6$-month) SD rate of $37 \%$. In the phase III GIST trial, median time to tumor progression was $>4$-fold as long with sunitinib as with placebo at an interim analysis (27.3 vs. 6.4 weeks; HR, $0.33 ; \quad P<0.001)$. These findings formed the basis for the US FDA approval - and the recent positive CHMP opinion for EU approval (April 2006) - of sunitinib for the treatment of advanced RCC and of GIST after disease progression on or intolerance to imatinib therapy. Several trials of other solid tumors are also underway or will soon begin recruitment. For example, preliminary data from phase II studies of sunitinib in metastatic breast cancer and unresectable neuroendocrine tumor have been promising, with partial response and SD rates of up to $13 \%$ and $93 \%$, respectively, observed in these two tumor types. Regardless of the tumor type, most adverse events have been of grade $1 / 2$ severity with grade 4 events uncommon. In conclusion, updates to the trials described above and preliminary data from sunitinib studies of potential biomarkers, other tumor types, and different treatment regimens (continuous dosing, 
first-line treatment in $\mathrm{mRCC}$, and combination therapy) will be presented.

\section{Presidential Symposium 5}

\section{Clinical overview of sorafenib: an oral multi-kinase inhibitor with activity in renal cell carcinoma, hepatocellular carcinoma, and malignant melanoma}

\section{Dimitris Voliotis}

\section{Bayer Health Care Pharmaceuticals, Germany}

Sorafenib was first identified as a potent in vitro inhibitor of Raf-1 kinase. However, it has since been shown to inhibit wild-type B-Raf and oncogenic b-raf V600E serine/threonine kinases, and several proangiogenic (VEGFR1/-2/-3; PDGFR- $\beta$; FGFR-1) or tumor promoting (c-Kit, Flt-3, RET, p38). Sorafenib acts on the tumor and the tumor vasculature to inhibit tumor growth by an anti-proliferative, proapoptotic, and/or anti-angiogenic effect in preclinical models of RCC, HCC, melanoma, and other cancers. In a Phase II randomized discontinuation trial, sorafenib $400 \mathrm{mg}$ twice daily (bid) demonstrated a significant median progression-free survival (PFS) advantage over placebo ( 24 vs. 6 weeks; $P=0.0087$ ) in a cohort of 202 patients with advanced RCC. This significant PFS benefit over placebo ( 24 vs. 12 weeks; $P<0.000001)$ was confirmed in a Phase III trial that was the largest performed to date $(n=903)$ in patients with advanced, clear cell RCC. This PFS benefit was maintained across all patient subgroups. Sorafenib also demonstrated a $39 \%$ improvement in overall survival (OS) according to a 220 event analysis $(P<0.018$; hazard ratio 0.72). Sorafenib was generally well tolerated with the majority of adverse events being grade $1 / 2$ and easily manageable. The most common events were dermatological (rash 40\%, hand-foot skin reaction 30\%), gastrointestinal (diarrhea $43 \%$ ) and constitutional (fatigue $37 \%$ ). Hypertension (mainly grade 1/2) was more common with sorafenib $(17 \%)$ than placebo $(2 \%)$. Sorafenib was approved by the FDA for the treatment of advanced RCC in December 2005. In a Phase II trial sorafenib $400 \mathrm{mg}$ bid induced partial $(2.2 \%)$ and minor $(5.8 \%)$ responses, or stable disease for $>16$ weeks $(33.6 \%)$ in patients with advanced HCC. Median time to progression (TTP) was 4.2 months, and median OS was 9.2 months. Grade 3/4 drug-related toxicities included fatigue $(9.5 \%)$, diarrhea $(8.0 \%)$, and hand-foot skin reaction $(5.1 \%)$. Sorafenib has also shown promising activity in combination with carboplatin and paclitaxel, or DTIC in Phase I trials. Sorafenib combined with carboplatin and paclitaxel was associated with an $85 \%$ clinical benefit $(n=1$ complete response $[<1 \%]$; $n=27$ partial responses [26\%]; $n=61$ stable disease [58\%]) in patients with advanced melanoma. The overall median PFS was 8.8 months (15.2 months for responders; $n=28)$. Sorafenib combined with DTIC induced three partial responses $(17 \%)$, and a further 11 patients $(61 \%)$ had stable disease. These sorafenib combinations were generally well tolerated. Phase III trials are currently accruing to further evaluate sorafenib in HCC and melanoma.

\section{Presidential Symposium 5}

\section{Clinical development of TAC-101 targeting retinoic acid receptor- $\alpha$}

Takashi Arima ${ }^{1}$, Melanie B. Thomas ${ }^{2}$, Yvonne Lassere $^{2}$, Karla Bogaard ${ }^{2}$, Thomas D. Brown ${ }^{2}$, Yehuda Patt $^{3}$, Paulo M. Hoff ${ }^{2}$, Koji Murakami ${ }^{1}$, James L. Abbruzzese $^{2}$

${ }^{1}$ Taiho Pharmaceutical Co., Ltd., Japan, ${ }^{2}$ The University of Texas M. D. Anderson Cancer Center, USA, ${ }^{3}$ The University of New Mexico, USA

Background $\mathrm{HCC}$ are resistant to chemotherapy; even transient disease stabilization is uncommon. TAC101 (4[3,5-bis (trimethylsilyl) benzamide] benzoic acid) is an orally-bioavailable synthetic retinoid that binds to nuclear retinoic acid receptor-alpha (RAR-alpha), activates RAR-alpha transcriptional activity. In animal models of liver cancer, antiangiogenic, antimetastatic, and life-prolongation activities were observed. Thus, a Phase I/II study was carried out in HCC patients (pts). Patients and methods Pts were treated with 2 courses of 14 days on/7 days off TAC-101. Initial dose for phase I cohort was $40 \mathrm{mg}$ daily. All pts receiving any study medication were evaluable for safety; those pts who received at least 28 doses ( 2 courses), were evaluable for efficacy. Response was evaluated every 6 weeks by WHO criteria. Results For all pts median age was $65 ; 22$ had received prior therapy: 18 chemo, 10 surgery, 4 RT, 1 immunotherapy, 2 other. Of the 5 pts treated at $40 \mathrm{mg}$ daily, 2 developed DLTs. Then dose was deescalated to $20 \mathrm{mg}$. Of the first $7 \mathrm{pts}$ treated at $20 \mathrm{mg}$ daily, of whom 6 are evaluable for dose tolerability, one developed DLT; therefore, $20 \mathrm{mg}$ daily was the MTD. Of the 28 pts treated at $20 \mathrm{mg}$ daily, $21 \mathrm{pts}$ were evaluable for efficacy. Pts received a median 2 courses; 9 pts received at least 4 courses of treatment. During treatment, no pt had a CR or PR. However 2 pts had PRs noted as significant necrosis, 8 and 11 months after starting, and 3 and 6 months respectively after discontinuing TAC-101, 
without further treatment. For all pts treated with $20 \mathrm{mg}$ daily, median time to treatment failure (TTF) was 6.6 weeks; 9 pts had TTF $>12$ weeks. One pt treated at $20 \mathrm{mg}$ daily developed a pulmonary embolism (PE); $1 \mathrm{pt}$ developed Grade (Gr) 3 VTE; 1 pt developed Gr 3 AST elevation for which drug was discontinued. Gr $>1$ toxicities occurring in $>10 \%$ of pts at $20 \mathrm{mg}$ daily included fatigue, myalgias and increased triglycerides, pruritus, dermatitis, AST increase, ALT increase and nausea, and anorexia. Conclusions $20 \mathrm{mg}$ TAC-101 for 14 days every 3 weeks was well-tolerated in pts with advanced HCC. Significant disease stabilization was noted in a moderate proportion ( $42 \%$ ) of this population, which is equivalent to or better than many cytotoxic chemotherapy agents; 2 late PRs may be attributable to TAC-101. Further investigation was warranted.

\section{Special Seminar 1}

\section{Current status of molecular targeted therapy for metastasis}

\section{Roy S. Herbst}

The University of Texas, MD Anderson Cancer Center, USA

The critical role of angiogenesis in tumor growth and metastasis is now well established in the literature. Growing tumors stimulate neovascularization through the secretion of pro-angiogenic growth factors, in particular, basic fibroblast growth factor (bFGF) and vascular endothelial growth factor (VEGF). Several lines of evidence have implicated VEGF in tumorigenesis and understanding the role of VEGF in tumor angiogenesis has facilitated the development of novel targeting agents that specifically interfere with angiogenesis. The different approaches to disrupt tumor-induced angiogenesis encompass tyrosine kinase inhibitor, monoclonal antibodies, small molecule inhibitors and transcription inhibitors. However, monoclonal antibody and tyrosine kinase inhibitors are the most advanced drug classes currently being investigated in clinical trials. To date, three anti-VEGF inhibitors namely Bevacizumab, Sunitinib and Sorafenib have been approved for the treatment of solid human malignancies including colorectal cancer, gastrointestinal stromal tumors and renal cell cancer. Additional anti-angiogenesis drugs are investigated in various types of cancer. This lecture will review the current clinical knowledge on the use of these agents to interfere with VEGF, VEGFR, matrix breakdown or other mechanisms involved in angiogenesis and tumor metastasis with a focus on NSCLC.

\section{Special Seminar 2}

\section{Molecular determinants and dynamics of tumor cell dissemination in cancer patients}

\section{Klaus Pantel}

Institut fur Tumorbiologie, Universitätsklinikum Hamburg-Eppendorf, Germany

Metastasis is the major cause of deaths in patients with solid tumors. Occult micrometastatic spread of tumor cells might be the seed for the occurrence of overt metastases in distant organs within the years following successful surgical resection of the primary tumor. The two major approaches to detect such "minimal residual disease" are immunocytochemical staining and polymerase chain reaction analysis. These assays are sensitive enough to detect a single disseminated tumor cell (DTC) in the background of millions of normal cells (Pantel and Brakenhoff, Nat Rev Cancer 4:1-9, 2004). For epithelial tumors, cytokeratins have become the best marker for the immunocytochemical detection of DTCs in blood, lymph nodes and bone marrow (BM).

Micrometastatic cells in BM can be easily collected from the iliac crest, and BM seems to be a common homing organ for DTCs derived from various types of malignant epithelial tumors (e.g., breast, lung, prostate or colorectal cancer). DTCs are present in BM samples of $20-40 \%$ of patients even in the absence of lymph node metastases (stage N0) or clinical signs of overt distant metastases (stage M0). A pooled analysis on 4,703 breast cancer patients [1] has shown that the detection of DTC in BM is a marker for an increased risk to develop metastatic relapse. The molecular and biochemical characterization of DTCs helps to identify progression markers towards overt metastases and new therapeutic targets for therapies that are specifically directed against minimal residual disease. This information may refine the current DTC detection approaches, which the major goal of the EU-funded consortium "DISMAL" coordinated by K.P.

\section{Reference}

1. Braun et al (2005) NEJM 353:793-802

\section{PL 1-1}

Matrix metalloproteinase targets in breast cancer bone metastasis 
Erik W. Thompson, Manisha Shah, Andrea Connor, Blick Tony, Mark Waltham

St.Vincent's Institute and University of Melbourne Dept. Surgery, Australia

Matrix metalloproteinases (MMPs) are important for bone remodeling and different family members are expressed in osteoblasts and/or osteoclasts. MMP-1,2,9,13 and 14 have been reported to play active roles in bone remodeling, with mice deficient in MMP-9, MMP-13 or MMP-14 (MT1-MMP) showing significant bone development phenotypes. We have found that Prinomastat (AG3340, Agouron Phizer), a semi-specific MMP (gelatinase) inhibitor, attenuates osteolytic bone metastasis generated in syngeneic and xenograft mouse models of breast-to-bone metastasis. Others have reported similar effects with other MMP inhibitors in mouse models of breast metastasis, as well as in osteolytic PC-3 prostate cancer cells. While this raises the possibility that MMP inhibitors could be used in the clinical treatment of cancer induced osteolysis, prior clinical experience with this class of drugs in oncology has not been particularly successful due to lack of inhibitor specificity within the MMP family. Given the need to refine MMP inhibition to more specific targets, we have employed speciesspecific qRT-PCR analysis in our xenograft model of bone metastases to determine the MMPs present in the tumoural (human) or stromal (mouse) compartments. We saw significant upregulation of stromal (mouse) MMP-2, MMP-11, MMP-13, and MT1- and MT3-MMP. We did not see evidence of murine MMP-9 or MT2-MMP. In the human (tumour) component, we saw minimal MMP expression. Targeting of these specific MMPs in the bone metastasis setting is ongoing. Unlike the prior clinical testing of MMP inhibitors which were primarily directed to primary tumour scenario, bone metastasis represents a late stage manifestation, and may therefore be a more fruitful environment for testing of MMP inhibitors.

\section{PL 1-2}

\section{Molecular mechanisms of solid tumor metastases to bone: therapeutic implications}

Theresa A. Guise

\section{University of Virginia, USA}

Certain solid tumors, such as breast and prostate cancer, have a propensity to metastasize to bone and cause osteolysis and abnormal new bone formation.
The respective phenotypes of dysregulated bone destruction and bone formation represent two ends of a spectrum and most patients will have evidence of both. The mechanisms responsible for tumor growth in bone are complex and involve tumor stimulation of the osteoclast and the osteoblast as well as the response of the bone microenvironment. Unique interactions between tumor cells and bone cells drive a vicious cycle to fuel tumor growth in bone and make bone the most favorable site for metastases by breast and prostate cancer. These interactions are targets for therapeutic intervention. The role of immune cell function in the pathophysiology of bone metastases, although largely undefined, is most likely substantial, given known roles of bone metastases factors in immune function.

\section{Role of TGF- $\beta$ in breast cancer osteolysis}

Breast cancer commonly metastasizes to and destroys bone to cause pain and fracture. Tumors produce abundant factors which stimulate osteolysis: PTHrP, IL-11, IL-8, IL-6 and RANKL. Substantial data support central roles for bone-derived transforming growth factor-beta (TGF- $\beta$ ) and tumor-derived osteolytic factors such as PTHrP and IL-11 in a vicious cycle of local bone destruction in osteolytic metastases. TGF- $\beta$, stored in bone matrix and released in active form during osteoclastic resorption, stimulates PTHrP and IL-11 production by tumor cells and mediates bone destruction by stimulating osteoclasts. These data provide proof of principle for TGF- $\beta$ blockade in the treatment of breast cancer bone metastases. SD-208, a small molecule inhibitor of TGF- $\beta$ receptor I kinase (TGF- $\beta$ RI), inhibits signaling downstream of the TGF- $\beta$ receptor and blocks $\operatorname{smad} 2 / 3$ phosphorylation. The in vivo efficacy of SD-208 was evaluated in a bone metastasis model using the breast carcinoma cell line MDA-MB-231. SD-208 reduced the development and progression of osteolytic bone destruction, as assessed by computerized image analysis of radiographs, and prolonged survival. SD-208 did not affect growth in vitro or at mammary fat pad. However, SD-208 inhibited TGF- $\beta$-stimulation of, IL-11, PTHrP, and growth promoting factor, connective tissue growth factor (CTGF). It also reduced TGF- $\beta$ secretion from MDA-MB-231 cells, suggesting possible autocrine effects of TGF- $\beta$ on the cancer cells. SD-208 also inhibited osteoclast activity, stimulated osteoblast differentiation in vitro and in vivo and increased bone mass at sites unaffected by metastatic tumor. Taken together, these data indicate that therapeutic targeting of TGF- $\beta$ may decrease the osteolytic bone metastases due to breast cancer by blocking tumor production of osteolytic and growth-promoting factors such as IL-11, PTHrP and CTGF as well as by having direct effects on bone cell function. 


\section{Role of ET-1 in osteoblastic bone metastases}

Prostate cancer metastasizes to bone and stimulates abnormal new bone formation. Although such metastases have been classified as osteoblastic based on the radiographic appearance of the lesion, it is clear that both bone resorption and bone formation are dysregulated. Thus, an understanding of the mechanisms of tumor-induced bone formation as well as the role of the osteoclast in this process is critical to improve therapy. Tumor production of factors such as platelet-derived growth factor, insulin-like growth factors and adrenomedullin have been implicated in osteoblastic bone metastases. The vasoactive peptide, ET-1, is produced by prostate cancer and simulates the new bone formation associated with osteoblastic metastases via its effects on the endothelin A receptor (ETAR) in mice and in humans. An ETAR antagonist (atrasentan) prevented osteoblastic bone metastases in a mouse model and reduced skeletal morbidity in men with advanced prostate cancer. These effects appear to be bone-specific as ETAR blockade reduces tumor growth in bone, but not at non-bone sites. ET-1 stimulates bone formation, in part, by suppression of the tonic inhibitor of bone formation, Dkk1, a member of the canonical Wnt signaling pathway. ET-1 also stimulates osteoblast production of factors such as $\mathrm{CCN}$ proteins and IL-6 which may further enhance tumor growth in bone.

The unique properties of the tumor and the bone make it a very favorable site for solid tumor metastases. Bone metastases are the result of complex interactions between tumor cells, bone cells and the bone microenvironment. Targeting these interactions should lead to effective treatment of this devastating complication of advanced cancer.

\section{PL 1-3}

\section{Bone metastasis of lung cancer and its molecular targeted therapy}

Seiji Yano ${ }^{1}$, Hiroaki Muguruma ${ }^{1}$, Shinsaku Ohtsuka ${ }^{1}$, Toyokazu Miki ${ }^{1}$, Hisanori Uehara ${ }^{2}$, Makoto

Kawatani $^{3}$, Hiroyuki Osada ${ }^{3}$, Saburo Sone ${ }^{1}$

${ }^{1}$ Department of Internal Medicine and Molecular Therapeutics, University of Tokushima Graduate School, Japan, ${ }^{2}$ Department of Molecular and Environmental Pathology, University of Tokushima Graduate School, Japan, ${ }^{3}$ Antibiotics Laboratory, Discovery Research Institute, RIKEN, Japan

Lung cancer is the major cause of malignancy-related death worldwide, and over one third of patients with advanced lung cancer develop osteolytic or osteoblastic bone metastasis. Bone metastasis causes severe symptoms and considerably reduces quality of life in lung cancer patients. Currently, no curative therapy exists for bone metastasis, and thus the prevention and treatment of bone metastasis are clinically important. We previously established an osteolytic bone metastasis model with a human small-cell lung cancer cell line, SBC-5, in natural killer cell-depleted SCID mice. SBC-5 cells highly expressed the parathyroid hormone-related protein (PTHrP) and produce experimental metastatic foci not only in the bone, but also in the visceral organs such as the lung, liver and kidneys, representing a patient-like metastasis pattern of lung cancer. Treatment with antiPTHrP Ab selectively inhibited the formation of osteolytic bone metastasis and improved the elevated serum calcium level, suggesting essential role of PTHrP on osteolytic bone metastasis and hypercalcemia in this model. Reveromycin A (RM-A), an inhibitor of isoleucyl-tRNA synthetase (IleRS), has a high potential to inhibit bone resorption by inducing osteoclast-apoptosis via the suppression of IleRS in osteoclasts. It suppressed the production of osteolytic bone metastasis by SBC-5 cells. Interestingly, it inhibited the expression of PTHrP by SBC-5 cells. Therefore, RM-A seems to be a unique agent that has activity toward both tumor cells and the host microenvironmental cells (osteoclasts). Bisphosphonates (Bis) are potent inhibitors of osteoclastic bone resorption, and have been widely used in the treatment of hypercalcemia. We found that monotherapy with the third generation Bis (zoledronate and minodronate) selectively suppressed the production of bone metastases, associating with decreased number of osteoclasts. However, it had no effect on visceral metastasis and combined use of Bis (minodronate) and chemotherapy (VP-16) was necessary to prolong the survival of SBC-5-bearing mice, providing the strategic rationale of combined use of Bis and chemotherapy for lung cancer patients. We recently established an osteoblastic bone metastasis model using human lung adenocarcinoma lung cells (ACC-LC-319). We will also discuss the therapeutic effect of bisphosphonate and VEGF inhibitors in both osteolytic and osteoblastic bone metastasis models.

\section{PL 1-4}

\section{Systemic analysis of the molecular network underlying breast cancer bone metastasis}

Yibin Kang, Guohong Hu, Mario A. Blanco, Heng Wu

Department of Molecular Biology, Princeton University, USA 
Metastasis represents the most devastating stage of cancer progression. Although previous functional genomics studies have identified a series of gene expression signatures that are associated with metastasis phenotypes, the global regulatory network underlying these tissue-specific metastasis gene profiles remained largely unexplored. We combined a series of novel computational approaches with in vivo and in vitro functional studies to identify and analyze the metastasis regulatory programs (MRPs) that link the emergence of bone metastasis gene expression profiles with altered activities of transcriptional factors and other regulators of cellular physiology. We used biclustering analysis to identify synexpression gene groups according to their expression concordance in 46 variants of MDA-MB-231 breast cancer cell line. The co-expressed genes that play significant roles in metastasis profiles are further identified by gene set enrichment analysis. Subsequently, transcription factor binding site enrichment analysis of promoter sequences was performed to identify the master regulator of metastasis-related synexpression groups. The function of putative MRPs in metastasis are currently been validated by functional studies of genetically modified human cells in mouse models of cancer metastasis and by clinical correlation assessment in human breast cancer samples. Results from this study will not only enrich our understanding about the molecular mechanism of metastasis, but also provide key targets for developing novel therapeutic and diagnostic approaches.

\section{PL 1-5}

\section{Myeloma expansion and bone destruction by the bone marrow microenvironment}

\section{Masahiro Abe}

Department of Medicine and Bioregulatory Sciences, University of Tokushima Graduate School of Medicine, Japan

Multiple myeloma (MM) is characterized by almost exclusive expansion within the bone marrow and progression of devastating bone destruction, suggesting critical roles for the bone marrow microenvironment in $\mathrm{MM}$ expansion and bone destruction. MM cells potently enhance both formation of OCs and resorption pits in osteoclastogenic cultures with bone marrow cells on dentine slices. MM cells from patients with osteolytic lesions secrete the osteoclastogenic C-C chemokines macrophage inflammatory protein (MIP)1 alpha and MIP-1 beta with positive correlation with the clinical severity of a bone disease; neutralizing antibodies against these factors in combination mostly abrogate in vitro $\mathrm{OC}$ formation and function enhanced by MM cells, indicating them as predominant mediators for osteolysis enhanced in MM. Besides its osteoclastogenic activity, MIP1 acts on MM cells in an autocrine/paracrine fashion to enhance adhesion of MM cells to VCAM-1 on stromal cells. Such adhesion in turn further up-regulates osteoclastogenic activity along with the levels of MIP-1 produced by MM cells, which may create a microenvironment with a close cell-cell interaction and high concentration of these chemokines in the bone marrow. Importantly, the growth and survival of MM cells are potently enhanced by contact with OCs, and MM cells become resistant to doxorubicin in the presence of OCs, suggesting the formation of a vicious cycle between MM cells and OCs. OCs produce a variety of anti-apoptotic factors for MM cells, including IL-6 and the TNF family members BAFF and APRIL. Interestingly, the MM-OC interaction also enhances angiogenesis largely through VEGF and osteopontin elaborated by MM cells and OCs, respectively. In sharp contrast to the enhanced osteoclastogenesis, MM cells suppress dendritic cell (DC) formation from monocytes, a common progenitor for OCs and myeloid DCs, and bone formation. Of note, most MM cells secrete inhibitors for a canonical Wnt pathway, an essential signaling pathway of osteoblast differentiation, including DKK-1 and sFRP-2. Thus, MM cells enhance bone resorption and suppress bone formation to cause bone destruction and rapid loss of bone. The interaction of MM cells with the bone marrow microenvironment gives rise to induction of OCs, vascular endothelial cells and immature stromal cells along with suppression of DC formation in the bone marrow, which favors MM cell growth and survival and enhances its drug resistance. Such skewed cellular constituents of the microenvironment can be defined as the MM niche, which should be targeted to prevent devastating bone destruction and tumor growth.

\section{PL 1-6}

\section{Cell-cell contact regulated genes and pathways in prostate bone metastasis formation}

Robert L. Satcher, Jun Wang, Jennifer Jarrett, Anait Levenson

Department of Orthopaedics and The Robert Lurie Comprehensive Cancer Center, Northwestern University, Chicago, IL, USA 
Prostate cancer is the most commonly diagnosed cancer in males in the US, and frequently spreads to bone. Metastases often start in metaphyseal bone, a microenvironment enriched with bone marrow stromal cells (BMSC). The molecular mechanism responsible for the crosstalk between bone and tumor cells remains largely unknown. Using gene-arrays to screen for regulated genes readily identifies hundreds to thousands of candidate genes. Consequently, choosing the gene(s) that should be further studied is challenging. What is needed is a more specific method. Our goal was to identify a small number of genes and pathways that are regulated by contact and mediate bone metastasis formation. For this, we combined co-culture with gene-arrays and clustering analysis to provide more specificity. Five metastatic prostate cell lines (three that metastasize to bone: PC3, C4-2; $\mathrm{PCa} 2 \mathrm{~b}$; one to lymph nodes-LNCaP, and one to brain-DU145) were individually co-cultured with BMSC. Metastatic tumor cells were co-cultured with BMSC allowing cell-cell contact; or with a separating barrier that only allowed soluble factor exchange. Fluorescence-activated cell sorting was performed to re-separate co-cultured cells. Expression was analyzed using custom made 'tumor metastasis' and 'osteogenesis' gene arrays to identify genes that were modified by cell-cell contact, and confirmed by RT-PCR. Gene clustering analysis was performed using the GeneSifter software-tool. From 192 genes, a total of twenty-five genes amongst the five metastastic cell lines were regulated during co-culture with BMSC. Fifteen genes were found to be modified in BMSC. Two pathways (one in metastatic cells and one in BMSC) were identified via clustering analysis that are likely involved with contact mediated crosstalk), with two upregulated genes (Mta-1 and MUC 1) associated with cancer progression. Cell lines showing similar patterns of contact mediated expression using clustering analysis were PC3, PCa2b, and C4-2 (all metastatic to bone). A small subset of genes (PDGF receptor, FGF receptor, and Raf1) was responsible for this clustering. These genes mediate the MAP kinase pathway, associated with proliferation and cell differentiation. In BMSC, a small subset, including TGFbeta receptor, decorin, and smads 6 and 7 was identified that mediates the TBFbeta signaling pathway associated with apoptosis and cell cycle. This study suggests that crosstalk may act through similar functional genes and pathways in any prostate cancer cells that metastasize to bone. Selecting genes for further study is made considerably easier by the improved specificity of the co-culture/gene-array method.
Supported by NCI-Spore and Robert Wood Johnson Grants to RLS.

\section{PL 2-1}

\section{New molecular diagnostic method for rapid detection of lymph node metastasis in breast cancer}

Nariaki Matsuura ${ }^{1}$, Masahiko Tsujimoto ${ }^{2}$, Kaduzki Nakabayashi $^{3}$, Motonari Daitho ${ }^{3}$, Junyi Ding ${ }^{3}$, Yasuhiro Otomo ${ }^{3}$

${ }^{1}$ Department of Molecular Pathology, Osaka University Graduate School of Medicine and Health Science, Japan, ${ }^{2}$ Department of Pathology, Osaka Police Hospital, Japan, ${ }^{3}$ Central Research Laboratories, Sysmex Corporation

Intraoperative accurate evaluation of lymph node status is essential for appropriate lymph node dissection for curative operation in cancer patients. Histopathological diagnosis to frozen section is usually used for this evaluation. However there have been reported some problems for intraoperative histological examination, including lower sensitivity due to observing only small portion of whole lymph nodes and dependency on pathologists' capabilities. The molecular analysis using RT-PCR (reverse-transcriptase polymerase chain reaction) has higher sensitivity and more objectivity, but clinical application for rapid evaluation during operation would be difficult since it takes several hours. To overcome these problems, we develop a new intraoperative molecular diagnostic method, one step nucleic acid amplification (OSNA), to measure cytokeratin (CK) 19 mRNA expressions quantitatively. This assay consists of the sample preparation and rapid gene amplification by RT-LAMP (reverse transcriptase loop mediated isothermal amplification) and it takes within $30 \mathrm{~min}$ for the whole process. Frozen 106 lymph nodes of 36 patients with breast cancer were retrospectively analyzed for CK19 mRNA expression levels to determine cutoff value. And as a prospective study fresh 120 lymph nodes of 37 breast cancer patients were analyzed to evaluate intraoperative performance of OSNA assay in comparison with histopathological and immunohistochemical studies. As a result histological negative lymph nodes had less than $3 \times 102$ copies of CK19 mRNA per reaction, while histological positive ones had over $1.0 \times 103$ copies per reaction. From these results, the cutoff value of CK19 mRNA copy number was determined as 500 copies per reaction in OSNA assay. By using this cutoff value, OSNA assay had $98.1 \%(104 / 106)$ concordance with histopathological evaluation for serial sections at $1.0-2.0 \mathrm{~mm}$ intervals; 
the sensitivity was $100 \%(22 / 22)$, and the specificity was $97.6 \%(82 / 84)$. In the prospective analysis there was $96.6 \%(112 / 116)$ concordance between the results of OSNA assay and intraoperative histopathological examination evaluated at $1.0-2.0 \mathrm{~mm}$ intervals; the sensitivity was $92.3 \%(24 / 26)$, and the specificity was $97.8 \%$ (88/90). Two false negative lymph nodes contained micrometastasis. In conclusion examination of CK19 mRNA by OSNA assay would be useful diagnostic tool for rapid detection of LN metastasis in breast cancer since CK19 mRNA expression levels by OSNA assay were well correlated with histopathological analysis for many sections.

\section{PL 2-0}

\section{Identification and characterization of Anakin, a novel metastasis efficiency modifying gene, through genometic analysis}

Nigel P. Crawford ${ }^{1}$, Xiaolan Qain ${ }^{2}$, Argyrios Ziogas ${ }^{3}$, Brenda Boersma ${ }^{4}$, Luanne Lukes ${ }^{1}$, Renard Walker ${ }^{1}$, Hoda Anton-Culver ${ }^{3}$, Stefan Ambs ${ }^{4}$, Doug Lowy ${ }^{2}$, Kent Hunter ${ }^{1}$

${ }^{1} \mathrm{NCI} / \mathrm{NIH},{ }^{2}$ Laboratory of Cellular Oncology, National Cancer Institute, USA, Bethesda, MD 20892, USA, ${ }^{3}$ Epidemiology Division, Department of Medicine, University of California, Irvine, Irvine, CA 92697, USA, ${ }^{4}$ Laboratory of Human Carcinogenesis, Center for Cancer Research, National Cancer Institute, National Institutes of Health, Bethesda, MD 20892, USA

There is increasing evidence that germline polymorphism alters metastatic propensity. Quantitative trait locus (QTL) analysis in the polyoma middle-T (PyMT) mouse mammary tumor model has identified a number of loci modulating metastasis. The focus of this study is 2600005C20Rik ("Anakin"), a gene located within a metastasis efficiency QTL locus that is a candidate causative gene. Anakin was identified through two independent experiments, the first of which involved yeast two-hybrid analysis to identify protein-protein interactions with the previously described metastasis modifier SIPA1. The human homolog of Anakin (KIAA0179) was found to bind the PDZ domain of SIPA1, a protein-protein interaction motif critical to the role of SIPAI in modulation of metastatic efficiency. This interaction was confirmed in vitro by co-immunoprecipitation, with these experiments also revealing that Anakin reduces Sipa1 GAP activity analogous to Aquaporin-2. A second series of seemingly unrelated experiments were performed to clarify the role of extracellular matrix
(ECM) genes in metastasis-predictive microarray expression signatures. The program WebQTL was used to define ECM expression QTLs (eQTLs) in PyMT-induced mammary tumors in AKXD recombinant inbred mice. An ECM eQTL was identified on proximal chr-17, the position of which approximates to a previously identified metastasis QTL. Expression of Anakin was highly correlated with ECM gene expression. Ectopic expression of Anakin in a metastatic mouse mammary tumor cell line resulted in dysregulation of ECM gene expression. Subcutaneous implantation of these cells into FVB/NJ mice caused a significant reduction in tumor growth kinetics, consistent with our earlier observations of a tumor growth modifier on chr-17. To further define the role of Anakin in tumor progression, the human Anakin SNP rs9306160 (1307C > T, P436L) was characterized in two independent cohorts: one population of 300 breast cancer patients from California (CA), and a cohort of 246 breast cancer patients from Maryland (MD). The variant allele correlated with improved outcome, and was associated with a lower frequency of metastatic disease $(P=0.006[\mathrm{CA}])$, lower primary tumor grade $(P=0.002[\mathrm{CA}]$ and $P=$ $0.047[\mathrm{MD}]$ ), and a higher frequency of progesterone receptor-positive primary tumors $(P=0.003[\mathrm{CA}]$ and $P=0.007[\mathrm{MD}])$. Kaplan-Meyer survival analysis showed that the wildtype allele was associated with a lower likelihood of survival $(P=0.025[\mathrm{MD}])$. These data implicate Anakin as a modifier of tumor growth and metastasis efficiency. Further analysis will be required to define the specific mechanism(s) by which Anakin modulates these processes.

\section{PL 2-3}

\section{Identification of the bona-fide marker of isolated tumor cell to predict metastasis in solid cancer}

Koshi Mimori, Yoshimasa Kosaka, Shigeo Hirasaki, Yoshiaki Kita, Kenji Ishikawa, Hiroshi Inoue, Masaki Mori

The Department of Surgical Oncology, Medical Institute of Bioregulation, Kyushu University, Beppu, Japan

Introduction In the recent two decades, the identification of isolated tumor cells (ITC), such as circulating cells in peripheral blood or disseminated tumor cells in bone marrow has been expected to be a new indicator to predict the recurrence and prognosis of cancer patients. However, any definitive conclusions have been made until today, because the number of 
cases were inadequate and methodologies to detect ITC were various among institutes. Materials and Methods We collected both bone marrow (BM) and peripheral blood (PB) from 1,007 patients of gastric cancer in the Central Hospital, National Cancer Center and both from 848 cases of breast cancer in the Kyushu Cancer Center. Our institute determined the ITC in PB and BM by the quantitative real-time RT-PCR with three epithelial cell markers, CEA, CK19, and CK-7. In addition, urokinase plasminogen activator (u-PA) receptor expression in breast cancer was examined, respectively. We confirmed the absence of CEA, CK-19, and CK-7 expressions in PB and BM in 29 non-malignant patients. Results (1) Gastric cancer; The positive rate of ITC was $46.0 \%$ and $30.5 \%$ in $\mathrm{PB}$ and $\mathrm{BM}$, respectively. However, the incidence of ITC (+) in gastric cancer is associated with neither any clinicopathologic factors nor recurrence, and those rates were observed equally among every clinical stages. (2) Breast cancer; 262 (35.4\%) cases of ITC $(+)$ in PB showed poorer disease free survival than 482 cases of ITC (-). According to the previous study, u-PAR detected by immunocytochemistry (IC) was established as the predictive marker for the prognosis of breast cancer. Therefore, we examined u-PAR expression by Taq-man RT-PCR in breast cancer, and found that both 140 cases of u-PAR (+) in BM and 306 cases of that in PB disclosed extremely shorter period of DFS and OS with statistical significance as the previous study by IC. Conclusion It is extraordinarily that the circulating and disseminated tumor cells were detected at every clinical stage by CEA, CK-19 and CK-7 expression among more than 1,000 cases of gastric cancer. As we revealed that $\mathrm{u}-\mathrm{PAR}$ gene can predict DFS and OS in breast cancer cases greatly better than epithelial markers, it is important to identify a precise marker to detect the bona-fide ITC that forms metastasis and worsens prognosis in solid cancer cases

\section{PL 3-1}

\section{The epigenetic reprogramming of tumor cells by the microenvironment}

Mary J. C. Hendrix ${ }^{1}$, Paul M. Kulesa ${ }^{2}$, Jennifer C. Kasemeier-Kulesa ${ }^{2}$, Jessica M. Teddy ${ }^{2}$, Naira V. Margaryan $^{1}$, Elisabeth A. Seftor ${ }^{1}$, Lynne-Marie Postovit $^{1}$, Richard E. B. Seftor ${ }^{1}$

${ }^{1}$ Children's Memorial Research Center, Feinberg School of Medicine and Robert H. Lurie Comprehensive Cancer Center of Northwestern University, ${ }^{2}$ Stowers Institute for Medical Research, 1000 East 50th Street, Kansas City, MO 64110, USA

Our long term goal is to understand the biological mechanisms underlying the bidirectional communication between metastatic tumor cells and their microenvironment(s) that ultimately result in cell fate determination, plasticity, and epigenetic reprogramming. Our short term goal is to identify the biological and molecular parameters associated with the epigenetic reprogramming of metastatic melanoma cells exposed to embryonic microenvironment(s). Therefore, our laboratory and collaborators developed unique, threedimensional (3D), organotypic, extracellular models, in addition to an in ovo chick embryonic model, for the study of the epigenetic influence of the microenvironment on multipotent metastatic melanoma cell plasticity. The 3D model developed to allow metastatic melanoma cells to be exposed to the microenvironment of human embryonic stem cells (hESCs), revealed that hESC microenvironments can dramatically influence the behavior of aggressive melanoma cells. Specifically, exposure of tumor cells to H1- or HSF-6hESC matrices induced a melanocyte-like phenotype with the ability to form colonies similar to hESCs. Furthermore, melanoma cells were less invasive after culture on hESC microenvironments. These findings demonstrate the utility of this 3D model for studying the unique factors deposited by hESCs and for investigating the epigenetic effects that stem cell microenvironments may have on tumor progression. Using an embryonic chick model, we explored the possibility of reverting the metastatic melanoma phenotype to its cell type of origin, the neural-crest-derived melanocyte. GFP-labeled adult human metastatic melanoma cells were transplanted in ovo adjacent to host chick promigratory neural crest cells and analyzed 48 and $96 \mathrm{~h}$ after egg reincubation. Interestingly, the transplanted melanoma cells do not form tumors. Instead, we find that transplanted melanoma cells invade surrounding chick tissues in a programmed manner, distributing along host neuralcrest-cell migratory pathways. The invading melanoma cells display neural-crest-cell -like morphologies and populate host peripheral structures, including the branchial arches, dorsal root and sympathetic ganglia. Analysis of a melanocyte-specific phenotype marker (MART-1) and a neuronal marker (Tuj1) revealed a subpopulation of melanoma cells that invade the chick periphery and express MART-1 and Tuj1. Our results demonstrate the ability of adult human metastatic melanoma cells to respond to chick embryonic environmental cues, a subset of which may undergo a reprogramming of their metastatic phenotype. This 
model has the potential to provide insights into the regulation of tumor cell plasticity by an embryonic milieu, which may hold significant therapeutic promise.

\section{PL 3-2}

\section{Role of chemokines/their receptors in the formation of cancer metastasis}

Ikuo Saiki $^{1,2}$, Keiichi Koizumi ${ }^{1}$, Kazuo Yasumoto $^{3}$

${ }^{1}$ Division of Pathogenic Biochemistry, Institute of Natural Medicine, University of Toyama, Toyama, Japan, ${ }^{2}$ The 21 st Century COE Program, University of Toyama, Toyama, Japan, ${ }^{3}$ Division of Surgical Oncology, Cancer Research Institute, Kanazawa University, Kanazawa, Japan

Chemokines are a family of small cytokines that primarily induce directed migration of hematopoietic cells through interactions with a group of seven transmembrane, $\mathrm{G}$ protein-coupled receptors. We here show two recent topics of our study on the involvement of chemokines and their receptors in the formation of bone metastasis of lung cancer and the metastasis of gastric cancer to peritoneum in in vivo model.

1. We first examined expression of chemokines by differentiating osteoclasts. CC chemokine ligand 22/macrophage-derived chemokine, CCL22 was selectively up-regulated in osteoclast-like cells derived from RAW264.7 cells and mouse bone marrow cells upon stimulation with RANKL. In addition, a human lung cancer cell line SBC-5 that efficiently metastasized to bone when intravenously injected into NK cell-depleted SCID mice was found to express CCR4. Stimulation of SBC- 5 cells with CCL22 induced cell migration. Immunohistochemical analysis of bone metastasis lesions demonstrated close co-localization of osteoclasts expressing CCL22 and SBC-5 cells expressing CCR4. Collectively, we present a model for CCR4/ CCL22 system in bone metastasis of lung cancer. First, circulating CCR4-positive tumor cells are arrested in the bone. Second, PTHrP produced by cancer cells activates osteoblasts and induce their expression of RANKL. Third, RANKL promotes osteoclast differentiation and production of CCL22. Finally, CCL22 further promotes localization and survival of CCR4 expressing tumor cells in the bone marrow.

2. We next examined expression of chemokine receptors in human gastric carcinoma cell line. NUGC4 cells, which were all highly efficient in generating malignant ascites in nude mice upon intraperitoneal inoculation, expressed CXCR4 at high levels and showed migratory and proliferate responses to its ligand CXCL12/stromal cell-derived factor 1 (SDF-1). We also demonstrated that AMD3100 (a specific CXCR4 antagonist) effectively reduced tumor growth and ascitic fluid formation in nude mice inoculated with NUGC4 cells. Additionally, we examined human clinical samples. Malignant ascites fluids from patients with peritoneal carcinomatosis contained high concentrations of CXCL12. Moreover, immunohistochemical analysis demonstrated that CXCR4positivity of primary gastric carcinomas significantly correlated with the development of peritoneal carcinomatosis. Collectively, our results strongly suggest that the CXCR $4 / \mathrm{CXC12}$ axis plays an important role in the development of peritoneal carcinomatosis from gastric carcinoma.

\section{References}

1. Nakamura ES, Koizumi K, Saiki I et al (2006) Clin Exp Metastasis in press

2. Yasumoto K, Koizumi K, Saiki I et al (2006) Cancer Res 66(4):2181-2187

\section{PL 3-2}

Protease requirements in cancer invasion in vitro and in vivo: from individual to collective cell migration

Peter Friedl

Rudolf Virchow Center for Experimental Biomedicine and Dept. of Dermatology, Univ. of Wuerzburg, Germany

Cancer cell dissemination and metastasis in vivo result from a diverse set of migration strategies including individual cells and multicellular strands and clusters, referred to as collective invasion. Using 3D collagen lattices and in vivo intravital microscopy of cancer cell invasion, we have reconstructed at high resolution the subcellular location of pericellular proteolysis during the migration process, the resulting ECM remodeling, and invasion mechanism. The findings show how cell invasion and proteolytic ECM remodeling form a functional unit to generate collective cell invasion along realigned tissue structures and further reveal novel compensation strategies that rescue single-cell migration after protease inhibitor-based treatment. 


\section{PL 3-4}

\section{A favorable recipient-discriminating effects on cancerous \\ and normal mesothelial cells with calponin h1, a multifunctional actin-binding protein}

\author{
Shun'ichiro Taniguchi, Michiko Takeoka, Akemi \\ Sugaya, Yoko Yanagisawa, Naoki Itano
}

Dept. of Molecular Oncology, Shinshu Univ. Graduate School of Medicine, Matsumoto, Japan

Cytoskeletal molecules regulate cell morphology, motility and adhesion, and those quantitative and/or qualitative alterations affect metastasis-related phenotypes. Calponin h1 $(\mathrm{CN})$, an actin-stabilizing protein that is also known to interact with signal mediators, such as PKC and ERK etc., is suppressed in vascular smooth muscle cells of malignant human tumors and in mesothelial cells by coexisting cancer cells. We generated calponin h 1-deficient $\left(\mathrm{CN}^{-1-}\right)$ mice, which exhibited fragility in blood vessels and peritoneal membranes. Peritoneal cancer dissemination as well as hematogenous metastasis occurred more easily in the $\mathrm{CN}^{-/}$mice than in wild-type mice (Cancer Res 2001). The abnormal morphology of peritoneal membrane surface was reverted by the exogenous introduction of $C N$ gene. When $\mathrm{CN}$ was over expressed in $\mathrm{CN}^{-/}$and normal peritoneal mesothelial cells, they were not damaged at all but rather acquired an ability to resist cancer-induced intercellular dissociation.

On the other hand, $\mathrm{CN}$ gene transfer into several transformed cell lines, such as leiomyosarcoma (JNCI 1999), resulted in a suppression of malignancy. In both $\mathrm{CN}^{--}$mice and immunodeficient nude mice, the $\mathrm{CN}$ gene, given to target both cancer cells and the mesothelial cells, suppressed the peritoneal dissemination of ip injected malignant tumor cells, and prolonged the survival rate of the hosts (Int J Cancer 2003; Clinical Cancer Res 2006). The multifunctional nature of $\mathrm{CN}$, as a machinery player of cytoskeleton and/or mediator of signal transduction, probably resulted in a favorable recipient-discriminating effect on cancerous and normal cells.

We have made various truncated and/or pointmutated forms of $\mathrm{CN}$ to understand biological functions of $\mathrm{CN}$, directing attention to the ability to regulate actin filaments, cell motility, invasiveness and growth. We identified a responsible region for stabilizing actin filaments, and obtained a truncated form to suppress tumor growth and activities of MMP-2 and -9. We believe that if we use adequate multifunctional molecules for therapy, it is possible to simultaneously suppress cancer phenotypes and protect normal cells from the attack of cancer cells. (This study was done in collaboration with Drs. Tomonori Ogura and Hiroaki Kobayashi, Gynecology and Obstetrics, Graduate School of Medical Sciences, Kyushu Univ.; Depts. Dermatology, Ophthalmology, Pathology, and Obstetrics and Gynecology, Shinshu Univ. School of Medicine)

\section{PL 3-5}

\section{A perspective on in vivo modeling: the study of ezrin and osteosarcoma metastasis}

\section{Chand Khanna}

Tumor and Metastasis Biology Section, Pediatric Oncology Branch and Comparative Oncology Program, Center for Cancer Research, National Cancer Institute, USA

The study of metastasis biology requires a reliance on in vivo modeling. The complexity of this biology cannot be entirely captured by one model alone. By understanding the strengths and weaknesses of a set of models, it becomes possible to choose the appropriate model(s) for particular problems or questions. With this modeling approach in mind, we have focused on the problem of metastasis in the osteosarcoma. Despite successful control of the primary tumor and adjuvant chemotherapy, death from pulmonary metastases occurs in over $30 \%$ of patients within 5 years. To define genetic determinants of pulmonary metastasis in osteosarcoma we have utilized a crossspecies comparative approach (murine, canine, and human) for gene identification and evaluation. Using this comparative approach we have identified the cytoskeleton linker, ezrin, to be associated with metastasis. Our work suggests that ezrin is necessary for metastasis in murine transplantable osteosarcoma and genetically engineered rhabdomyosarcoma models, that it is relevant in human-murine sarcoma xenograft models, and that its expression is associated with metastatic progression in pet dogs with naturally occurring osteosarcoma. The translation of this data to the human condition has included our finding of an association between ezrin expression and risk of relapse in pediatric osteosarcoma patients. Through both in vitro and in vivo modeling we have recently determined that ezrin acts a biological node or nexus in the metastatic phenotype of cancer by facilitating signaling of many vital metastasis-associated pathways and by enabling the translational machinery of the cell. The use of single cell imaging within our mouse models has defined a role for ezrin in the protection of cells from apoptotic death early after their arrival 
in the lung and in the efficient transit of cells from the vessel to the lung parenchyma. These single cell studies have provided a biological screen for potential protein targets modulated by the ezrin-enabled translational machinery. Preclinical studies first in mice and then in dogs with naturally occurring osteosarcoma are underway to test inhibitors of these targets during metastasis. Our understanding of the biology of ezrin in metastasis and the evaluation of future therapeutic opportunities related to this biology has been facilitated through the use of a several models of metastasis. Each model system has been selected by the questions that can and cannot be asked of each model (modeling limits). Through this approach we have begun to understand the role of ezrin in the biology of osteosarcoma metastasis.

\section{PL 3-6}

\section{Lymphangiogenesis and cancer}

\section{Hajime Kubo}

HMRO, Graduate School of Medicine, Kyoto University, Japan

Blood and lymphatic vessels form a circulatory system, which allows the transportation of metabolic substances, cells and proteins in the body. The unidirectional lymph flow recovers extravasated tissue fluid from the periphery by blind-ended lymphatic cardiovascular system via the thoracic duct, which in turn drain into the venous circulation. During embryogenesis, the development of lymphatic vessels starts after the establishment of blood vasculature. A subset of venous endothelial cells commits to the lymphatic endothelial cell lineage and sprouts from the major veins in the jugular and perimesonephric area to form lymphatic sacs, from which the vessels grow further by centrifugal sprouting. The homeobox transcriptional factor prox 1 is required for the initial specification, while activation of VEGFR-3 by VEGF-C is essential for the survival, migration and proliferation of lymphatic endothelial cells. Recent studies also demonstrate that VEGFR-3 and its ligands are involved in certain pathological conditions, such as lymphatic metastasis. Recent progress in lymphangiogenesis will be demonstrated in this seminar in order of the topics listed below. (1) Antilymphangiogenesis for lymph node metastasis. (2) Differentiation of lymphatic endothelial cells from ES cells. (3) The expression and function of podoplanin in cancers. (4) Prox 1 as a tumor suppressor references: (1) Shimizu K, Kubo H, Yamaguchi K, Kawashima K, Ueda Y, Matsuo K, Awane M, Shimahara Y, Taka- bayashi A, Yamaoka Y, Satoh S (2004) Suppression of VEGFR-3 signaling inhibits lymph node metastasis in gastric cancer. Cancer Sci 95:328-33, (2) Kono T, Kubo H, Shimazu C, Ueda Y, Takahashi M, Yanagi K, Fujita $\mathrm{N}$, Tsuruo $\mathrm{T}$, Wada $\mathrm{H}$, Yamashita JK (in press) Differentiation of lymphatic endothelial cells from Embryonic Stem cells on OP9 stromal cells. Arterioscler Thromb Vasc Biol, (3) Shimoda M, Takahashi M, Yoshimoto T, Kono T, Ikai I, Kubo H (in press) A homeobox protein prox 1 is involved in the differentiation, proliferation and prognosis in hepatocellular carcinoma. Clin Cancer Res In press.

\section{PL 3-7}

\section{The intersection of gene expression and constitutional susceptibility to metastasis}

Kent W. Hunter, Nigel Crawford, Luanne Lukes, Renard Walker

Laboratory of Population Genetics, National Cancer Institute, National Institutes of Health, USA

Tumor metastasis is one of the most important clinical aspects of neoplastic disease since patient mortality is frequently due to disseminated, rather than primary tumors. However, in spite of the importance of this process and the significant investment of resources to investigate it, great gaps remain in our understanding of the mechanisms of metastasis. To gain a better understanding of metastasis, our laboratory has investigated how an individuals inheritance influences the propensity of a tumor to disseminate. Using a highly metastatic transgenic mouse mammary tumor model we demonstrated the presence of polymorphic loci in the genome that had a significant impact on the ability of tumors to colonize the lung. Subsequently, using a variety of methodologies, we have identified the first of these polymorphic genes, Sipa1, and demonstrated that modest changes in gene expression or function strongly impact metastatic efficiency. More recently, we have been investigated the origins of the recently described tumor-derived metastasis-predictive gene expression signatures. Exploration of our mouse model has demonstrated that many of the genes comprising these signatures are differentially expressed in normal tissue as well as tumor tissue, suggesting that constitutional polymorphisms, rather than just somatic events, are a major factor influencing the predictive expression patterns. Genetic mapping of the polymorphisms controlling the expression pattern of the metastasis predictive signature genes has revealed loci that co-localize with our previously mapped metastasis efficiency modifier 
loci. Further analysis of these loci has identified a number of polymorphic genes, that when ectopically expressed in a highly metastatic mammary tumor cell line, alter both the expression patterns of the endogenous metastasis-predictive signature genes and progression and metastasis after subcutaneous implantation in mice. These results suggest that genetic polymorphism is a significant factor modulating both metastatic efficiency and the metastasis-predictive gene expression patterns. If true, then SNP typing may prove to be an alternative or complementary method to expression analysis for clinical prognosis of tumor progression.

\section{PL 3-8}

\section{Modelling early steps of organ specific metastasis formation}

Peter Gassmann, Andre Hemping-Bovenkerk, Andreas Enns, Joerg Haier

Department of General Surgery, University Hospital Muenster, Muenster, Germany

Introduction The liver and the lung are preferred metastasis target organs for colon cancer and a variety of other malignant tumors. Therefore specific interactions of tumor cells and metastasis target organs have been hypothesised. We developed a model to visualise early organ specific events of liver metastases formation by fluorescence in vivo microscopy. Materials and Methods In vivo fluorescence microscopy of the liver, lung, kidney, mesentery, muscle and skin was performed in male CD rats $(250 \mathrm{~g} 300 \mathrm{~g})$ until $30 \mathrm{~min}$ after tumor cell injection. Human colon carcinoma cells with different metastatic potential were used (HT-29P $n=10$, HT-29 LMM $n=9$, KM12C $n=9$, KM-12 L4 $n=9)$. The cells were fluorescence labelled with Calcein AM and $10^{6}$ cells were injected intra-cardially. The number of adherent cells was semi-quantitatively analysed as described before and is expressed as adherent cells per 30 microscopic fields (MF). For localisation of cells within the hepatic parenchyma, cells and animals were marked with fluorescence dye suitable for formaldehyde fixation. Results Fluorescence labelled cells (HT 29LMM) could pass the microcirculation of all examined organs without mechanical arrest by size restriction. Cells showed specific adhesive interactions within the hepatic sinusoids and within the pulmonary capillaries. At the end of the observation period in the liver $22 \pm 12$ cell/30MF and in the lung $20 \pm 6$ cells/ 30 microscopic were observed (HT 29LMM). In most cases residual perfused micro vessel lumen could be seen. Within the renal capillaries mesentery, muscle and skin only very few adherent cells were found $(P<0.0001, t$-test $)$. In the liver it was possible to distinguish between cells localised within the hepatic sinusoids or migrated into the parenchyma by in vivo microscopy. The localisation within the hepatic parenchyma was verified in paraffin-embedded tissue by $3 \mathrm{D}$ confocal laser microscopy. Cells with increasing metastatic potential showed increasing migration rates until the end of the $30 \mathrm{~min}$ observation period: HT 29P: $16 \pm \%$; HT 29 LMM: $29 \pm 15 \%$; KM12C: $38 \pm 7 \%$; $\mathrm{KM} 45 \pm 5 \%(P<0.05 ; t$-test $)$. Conclusions Using in vivo microscopy we were able to establish a model to quantify tumor cell adhesion and extravasation in vivo. Colon carcinoma cell adhesion was only seen in typical metastatic target organs of human colon carcinoma and appears to be mediated by specific tumor cell-host organ interactions within the liver the rate of early tumor cell extravasation correlates with the liver metastatic potential of different cell lines, suggesting a rate limiting step within the metastatic cascade.

\section{PL 4-1}

\section{Inflammatory angiogenesis as a target for cancer therapy and prevention}
A. Albini ${ }^{1}$, N. Ferrari ${ }^{1}$, F. Tosetti $^{1}$, G. Fassina ${ }^{1}$, R. Benelli ${ }^{1}$, R. Vene ${ }^{1}$, C. Ambrosini ${ }^{1}$, R. Dell'Eva ${ }^{1}$, U. Pfeffer ${ }^{1}$, D. M. Noonan ${ }^{1}$
${ }^{1}$ National Cancer Research Institute, Genova, Italy, ${ }^{2}$ Universityof the Insubria, Varese, Italy

It is well known that a continuous cell proliferation can promote carcinogenesis and many tumors indeed occur in organs subdued to the chronic activation of those inflammatory responses that accompany tissue repair. A growing scientific evidence points tumor microenvironment as a key element in cancer onset and progression, and a possible target for therapy. The harmonized dialogue between endothelium and leucocytes mediates not only tumor onset, survival and growth, but also its ability to disperse through the blood stream. Several clinical trials have shown that angiogenesis is a promising, but also elusive, therapeutic target. Present approaches based on VEGF neutralization can have an adjuvant role in standard cancer therapy, but cannot be used per se, and the same idea of applying a high cost, short term, anti-angiogenic strategy against a long-term established pathology is misleading. Tumor progression shows very long latencies and the limitation of angiogenesis and inflammation at the beginning of the pathologic process, or as a target of secondary prevention, could 
offer better clinic performances if drugs with limited collateral effects and costs are identified.

We named "Angioprevention" a strategy based on the use of molecules sharing the ability to inhibit inflammation and angiogenesis in a chemopreventive setting. $N$-acetylcysteine (NAC), the green tea flavonoid epigallocatechin-3gallate (EGCG), the chalchone Xanthomol and the rotenoid Deguelin all prevent angiogenesis in vivo and inhibit the growth of angiogenic tumors in nude mice. The synthetic retinoid 4-hydroxyfenretinide (4HPR) also harbors anti-angiogenic effects, though using different mechanisms. In fact Affymetrix GeneChip array analyses of gene expression regulation by some of these compounds in primary endothelial cells (HUVEC) identified overlapping sets of genes regulated by the anti-oxidants. In contrast, the ROS-producing 4HPR induced members of the TGF $\beta$ ligand superfamily, which, at least in part, explain it's antiangiogenic activity. NAC and the flavonoids all suppressed the $\mathrm{IkB} / \mathrm{NF}-\kappa \mathrm{B}$ signaling pathway, even in the presence of TNF-alpha, and reduced expression of many NF- $\kappa \mathrm{B}$ target genes. Repression of the NF- $\kappa \mathrm{B}$ pathway suggests antiinflammatory effects for the antioxidant compounds that may also represent an indirect role in angiogenesis inhibition. The green tea flavonoid EGCG does target inflammatory cells, mostly neutrophils, and inhibits inflammation-associated angiogenesis. Our molecules are turning out to be also effective modulators of phagocyte recruitment and activation, further linking inflammatory angiogenesis to tumor onset and progression.

\section{PL 4-2}

\section{The essential roles of macrophages in angiogenesis and tumor growth: linking inflammation and cancer}

\section{Mayumi Ono}

Department of Medical Biochemistry, Graduate School of Medical Sciences, Kyushu University, Fukuoka, Japan

Microenvironmental changes in tumor stroma play key roles in malignant progression of cancer including angiogenesis, tumor growth and metastasis. In particular, a recent highlight is the microenvironmental changes by inflammatory stimuli, which could modulate malignant characteristics of various tumor types. In our present study, we focused on how inflammatory cytokines and infiltrated macrophages could promote angiogenesis and tumor growth by cancer cells.
(1) A representative inflammatory cytokine, IL-1 $\beta$ markedly induced angiogenesis in mouse corneas, and administration of COX2 inhibitors blocked the IL-1 $\beta$-induced angiogenesis, suggesting the involvement of COX2 pathway in the inflammatory angiogenesis [1].

(2) IL-1 $\beta$-induced angiogenesis was significantly curtailed when assayed in MCP-1-knock out mice, and also when treated with macrophage-targeting drug, bisphosphonate-liposome, suggesting the essential role of activated macrophages in the inflammatory angiogenesis [2].

(3) Angiogenesis as well as cancer growth was specifically reduced in tumor producing IL- $1 \beta$ when macrophages were depleted by administration of bisphosphonate-liposome, suggesting the involvement of activated macrophage in tumor angiogenesis [3]. Taken together, recruitment of macrophages into tumors in response to MCP-1 and other chemokines could affect tumor microenvironments, resulting in providing a favorable chance for cancer cells to growth and metastasis. We also discuss how macrophages are activated in response to inflammatory stimuli at molecular basis.

1. Kuwano T, Nakao S, Yamamoto H, Tsuneyoshi M, Yamamoto T, Kuwano M, Ono M (2004) Cyclooxygenase 2 is a key enzyme for inflammatory cytokinase-induced angiogenesis. FASEB J 18:300310

2. Nakao S, Kuwano T, Tsutsumi-Miyahara C, Ueda S, Kimura YN, Hamano S, Sonoda K, Saijo Y, Nukiwa T, Strieter RM, Ishibashi T, Kuwano M, Ono M (2005) Infiltration of COX2-expressing macrophage is prerequisite for IL- $1 \beta$-induced neovascularization and tumor growth. J Clin Invest 115:2979-2991

3. Kimura YN, Ono M (paper in preparation)

\section{PL 4-3}

\section{Tumor lymphangiogenesis and metastasis}

Terhi Karpanen, Yulong He, Kari Alitalo

Molecular/Cancer Biology Laboratory, University of Helsinki, Helsinki, Finland

In many human cancers, expression of the lymphangiogenic growth factor vascular endothelial growth factor C (VEGF-C) positively correlates with vascular invasion, 
lymphatic vessel and lymph node involvement, distant metastasis and, in some instances, poor clinical outcome. Also studies of various experimental tumor models have shown that VEGF-C or the related VEGFD enhance lymphatic metastasis, while a soluble form of their receptor VEGFR-3 inhibits tumor spread via the lymphatic vasculature. Although these experiments provide strong support for the involvement of VEGF-C and VEGFR-3 in the lymphatic spread of tumors, the underlying mechanisms have only recently been addressed.

While intratumoral lymphatic vessels have been detected in some solid human cancers, such as melanomas and head and neck carcinomas, xenotransplantation experiments have suggested that these might be poorly functional and may in fact not be needed for enhanced metastasis. On the other hand, the pressure gradient and lymphatic vessels at the tumor margin, stimulated to sprout by tumor secreted VEGF-C or VEGF-D, may be more important in spreading tumor cells. Our results show that the lymphatic endothelial cells at the tumor periphery send long filopodia towards the growth factor producing tumor cells and then form tumor-directed vessel sprouts, opening up the vessel lumen, which may allow facilitated access for tumor cells into the lymphatic vessel. Furthermore, the collecting lymphatic vessels, which drain fluid from the tumor area are stimulated to dilate by intraluminal VEGF-C via the process of endothelial proliferation in the vessel wall. Clumps of metastatic tumor cells could then be easily transported along with the lymph flow in the dilated hyperplastic vessels. Sequestering VEGF-C and VEGFD with the soluble VEGFR-3 decoy inhibited lymphatic vessel sprouting and dilation, and seemed to restore the integrity of the vessel wall. Similarly, blocking monoclonal antibodies that target VEGF-C, VEGF-D or VEGFR-3 and small molecules that inhibit the tyrosine kinase catalytic domain of VEGFR-3 could be used for the inhibition of tumor metastasis. Further work should soon tell if these same molecules inhibit further systemic metastasis or angiogenesis in tumor models.

\section{PL 4-4}

\section{Vascular endothelial growth factor (VEGF)-C and VEGF receptor-3 signaling directly and indirectly modulates murine mammary tumor growth and metastasis}

Rakesh Singh, Michelle Varney, Anguraj Sadanandam, Joyce Solheim, James Talmadge

Department of Pathology and Microbiology, University of Nebraska Medical Center, Omaha, NE, USA

At the time of diagnosis, the majority of breast cancer patients have developed lymph node metastases, which function as an important prognostic indicator. Recent studies in animal models suggest that lymphangiogenesis can promote metastasis. It has recently been shown that the lymphangiogenic growth factor, vascular endothelial growth factor-C (VEGF-C), and its receptor, VEGF receptor-3 (VEGFR3), may play a pivotal role in the promotion of metastasis to regional lymph nodes. Our recent study demonstrates that the inhibition of constitutive VEGF-C expression in murine mammary tumors reduces lymphangiogenesis and spontaneous metastasis and increases survival. In this study, we examined whether inhibition of VEGFR-3 modulates mammary tumor progression and metastasis. Cl66, a metastatic mammary tumor cell line was injected in the mammary fat pad of BALB/c mice. MAZ51, a VEGFR-3 receptor antagonist (with no effect on VEGFR-2) was injected intraperitoneally at different doses. We observed significant inhibition of tumor growth in a dose-dependent manner in mice treated with a VEGFR-3 antagonist as compared to mice injected with vehicle control. The number of spontaneous lymph node and lung metastases was also inhibited following treatment with VEGFR-3 antagonist. We did not observe significant different in experimental lung metastasis between VEGRR-3 treated and control vehicle treated group. We examined the mechanism(s) of VEGFR-3-mediated inhibition of tumor growth. Our data suggest that murine mammary tumor cells constitutively express VEGFR-3, suggesting that VEGFR-3 may have a direct effect on murine mammary tumor cells. In vitro treatment of Cl66 cells with VEGFR-3 antagonists inhibited cell proliferation, cellular adhesion and survival in a concentration dependent manner. Similar to our earlier studies suggesting that inhibition of VEGF-C modulates immune cell infiltration, we observed enhanced recruitment of $\mathrm{CD} 8+\mathrm{T}$ cells and DC1 dendritic cells in mammary tumors following treatment with a VEGFR-3 antagonist. Together these studies suggest that VEGF-C-VEGFR-3 signaling directly (affecting tumor cell phenotype) and indirectly (immune cell recruitment) modulates mammary tumor growth and metastasis.

\section{PL 4-5}

\section{The novel role of K-RAS signaling in the induction} of VEGF during hypoxia in colon cancer

Yusuke Mizukami ${ }^{1}$, Yutaka Kohgo ${ }^{1}$, Daniel C. Chung ${ }^{2}$ 
${ }^{1}$ Division of Gastroenterology and Hematology/ Oncology, Department of Medicine, Asahikawa Medical College, Hokkaido, Japan, ${ }^{2}$ Gastrointestinal Unit, Massachusetts General Hospital and Harvard Medical School, USA

Background The induction of VEGF is an essential feature of tumor angiogenesis. Hypoxia is a potent stimulator of VEGF, and hypoxia inducible factor-1 (HIF-1) is considered to be critical for its induction. However, we previously demonstrated that there are also HIF-1-independent pathways for hypoxic induction of VEGF that can be enhanced by $K-R A S$ signaling. In addition, the full spectrum of the effects of HIF inhibition on tumor angiogenesis and growth are not fully defined. Methods HIF-1 $\alpha$ was silenced in the colon cancer cell lines DLD-1 and Caco 2 by stable transfection of specific siRNA constructs. Changes in gene expression patterns induced by hypoxia and HIF- $1 \alpha$ silencing were evaluated by cDNA microarray, and these changes were confirmed by quantitative RT-PCR and ELISA. In vivo effects on angiogenesis were evaluated in nude mice utilizing HIF-1 knockdown DLD-1 cell (DLD-1 ${ }^{\text {HIF-kd }}$ ) xenografts. To define the HIF-1independent regulation of VEGF in hypoxic conditions, VEGF promoter reporter assays and gel electrophoretic mobility shift assays were performed. Results When DLD-1 ${ }^{\text {HIF-kd }}$ cells were grown as xenografts, VEGF was also induced, albeit at lower levels, and the expression of VEGF correlated with regions of intratumoral hypoxia even in the absence of HIF-1. Although silencing of HIF- $1 \alpha$ severely impaired tumor growth, the xenografts remained highly vascularized with microvessel densities that were identical to DLD-1 HIF-kd tumors. The pro-angiogenic cytokine interleukin8 (IL-8) was preferentially induced by hypoxia in DLD$1^{\text {HIF-kd }}$ cells. This induction of IL-8 was mediated by an increased production of hydrogen peroxide, resulting in the activation of NF- $\kappa \mathrm{B}$. $K-R A S$, which is commonly mutated in colon cancer, enhanced the production of IL-8 in $\mathrm{Caco}_{2}{ }^{\mathrm{HIF}-\mathrm{kd}}$ and DLD-1 ${ }^{\mathrm{HIF}-k d}$ cells. A novel promoter element at-271 bp of the VEGF promoter was responsive to hypoxia in a HIF-1-independent manner. c-Myc bound to this element, and $K-R A S$ enhanced the induction of VEGF by c-Myc through PI3K-Rho-Rock pathway. Conclusions Inhibition of HIF-1 by siRNA does not block tumor angiogenesis in colon cancer xenografts. Hypoxia persistently induces VEGF even in the absence of HIF-1 and c-Myc at least partially mediates this regulation. In addition, an alternative angiogenic pathway involving NF- $\kappa \mathrm{B}$ and IL-8 was induced when HIF- $1 \alpha$ was knocked-down. These findings emphasize the interplay between oncogenic signaling pathways and hypoxia, and highlight the pivotal role of $K-R A S$ in angiogenesis in colon cancer.

\section{PL 4-6}

\section{Thrombospondin-1/FGF-2 interaction in angiogenesis and tumor progression}

Giulia Taraboletti

Department of Oncology, Mario Negri Institute for Pharmacological Research, Bergamo, Italy

The endogenous inhibitor of angiogenesis thrombospondin-1 (TSP-1) is a matricellular molecule composed by distinct functional domains. The modular nature confers to TSP-1 the ability to bind simultaneously to soluble factors, cell receptors and matrix components, hence acting as a complex extracellular regulator of the cell interaction with the environment. Beyond its many potential activities, the final role of TSP-1 in a given biological setting is dictated by the cell types and soluble factors present in the environment. Aim of our study has been to investigate the functional interplay between TSP-1 and FGF-2 in the context of angiogenesis and tumor progression. TSP-1 binds with high affinity to the angiogenic factor FGF-2, inhibiting FGF-2 binding to endothelial cells and the extracellular matrix, hence modulating the growth factor bioavailability and activity. Using recombinant segments of TSP-1 spanning the entire $140 \mathrm{kDa}$ fragment with partial overlaps we located the FGF-2 binding region in the TSP-1 carboxyterminal cassette. Surface plasmon resonance analysis on BIAcore confirmed that the high affinity of this region for FGF-2. Binding of FGF-2 to TSP-1 and to the recombinant fragment was modulated by heparin and, consistently with the presence of the calciumbinding domain, by calcium concentrations. These findings locate the FGF-2 binding site within the carboxyterminal cassette of TSP-1, in a region different from the well-known anti-angiogenic type I repeats, hence indicating a new potential angiogenesis regulatory sequence of TSP-1. We investigated the role of TSP in melanoma progression. In most tumor types, the acquisition of the angiogenic ability that marks the switch to a malignant tumor, is associated to loss of TSP-1 production. In contrast, TSP-1 is produced by melanoma cells but not by melanocytes. The regulation of TSP-1 production by several factors associated with the progression of melanoma, including FGF-2, has been investigated in melanocytes and a panel of melanoma lines obtained from patients at different phase of progression. The findings highlight a role for FGF-2 in 
regulating TSP-1 production by melanoma cells, and suggest that TSP-1 production is associated with malignant progression in this neoplasia. (Supported by grants from AIRC and the EU-FP6, LSHC-CT-2003503233)

\section{PL 4-7}

\section{An endothelium-derived angiogenesis inhibitor vasohibin and its significance in tumor angiogenesis}

\section{Hikaru Sonoda}

Discovery Research Laboratories, Shionogi \& Co., Ltd. Osaka, Japan

Negative feedback regulation is one of the most important mechanisms in order to maintain homeostasis in our bodies. We have found the novel feedback regulator secretory protein for angiogenesis from gene expression profiling of endothelial cells stimulated by vascular endothelial growth factor (VEGF) and designated it as "vasohibin-1". Recombinant vasohibin-1 protein inhibited growth, migration and network formation of human umbilical vein endothelial cells. It also suppressed angiogenesis in vivo both in mouse corneal assay and chicken chorioallantoic membrane assay. The expression of vasohibin-1 gene is continuously inducible by VEGF and highly specific to endothelial cells. The characteristic amino acid primary structure deduced from cDNA sequence is the absence of hydrophobic signal sequence in its amino terminal and the presence of basic domain in its carboxyl terminal. Multiple processed forms of vasohibin-1 protein were observed in endothelial cells. Analysis of these processed forms revealed domains necessary for efficient secretion, heparin-binding and antiangiogenic activities.

In many tumor tissues, aberrant angiogenesis is caused by the angiogenic factors such as VEGF produced by tumor cells. We have studied the expression of hypoxia-inducible factor- $1 \alpha$ (HIF- $1 \alpha$ ), VEGF and vasohibin-1 in tumor tissues from lung cancer patients by immunohistochemistry, and found the strong association between the expression of HIF- $1 \alpha$ or VEGF and the ratio of vasohibin-1-positive blood vessels. These results suggested that vasohibin-1 is expressed in newly generated blood vessels in a tumor tissue and could be a possible surrogate marker for tumor angiogenesis. In fact, vasohibin-1-specific band was detected in Western blot analysis of plasma from these patients.

Recently we have identified the paralogous gene for vasohibin-1 which has also antiangiogenic activity and named it vasohibin-2. As observed for vasohibin-1, vasohibin-2 gene is strongly expressed in endothelial cells of embryonic tissues, but the expression is not induced by angiogenic factors tested so far, suggesting that these two family member proteins may play collaborative roles in angiogenesis in tissue development responding to distinct circumstances. From the view point of angiogenesis marker commented above, vasohibin-1 rather that vasohibin-2 might be a preferable candidate.

\section{PL 4-8}

Vascularization of melanoma by mobilization and remodeling of preexisting latent vessels to patency

Alan J. Schroit, Weixin Lu

Dept. of Cancer Biology, MD Anderson Cancer Center, USA

Tumors must manipulate the host vasculature to provide a blood supply adequate for their proliferation. Although tumors may arise as avascular masses, there is increasing evidence that some tumors begin to proliferate by first co-opting pre-existing host blood vessels. By fluorescent vascular imaging, we provide evidence that the vasculature in orthotopically-implanted melanoma arises from a preexisting red cell-deficient vascular network that remodels to patency to accommodate the requirements of the expanding tumor mass. Topical application of vascular endothelial growth factor (VEGF) to vascular beds generated immediate and robust vascular transitions that were morphologically similar to tumor-induced transitions. $N$-nitro-L-arginine, a nitric oxide inhibitor, significantly inhibited the growth of a syngeneic K1735M2 melanoma by reducing blood supply to the tumor by a mechanism independent of endothelial cell proliferation. These findings suggest that tumor-induced remodeling of red cell-deficient vessels to patency contributes to tumor vascularization and growth.

\section{PL 4-9}

\section{Inhibition of pericyte and reactive stroma by PDGF-R tyrosine kinase inhibitor reduces growth and metastasis of human colon carcinoma}

Yasuhiko Kitadai ${ }^{1}$, Takamitsu Sasaki ${ }^{2}$, Toshio Kuwai ${ }^{3}$, Toru Nakamura ${ }^{3}$, Corazon D. Bucana ${ }^{3}$, Isaiah J. Fidler $^{3}$

${ }^{1}$ Department of Medicine and Molecular Science, Hiroshima University, Hiroshima, Japan, ${ }^{2}$ First Department of Surgery, Fukuoka University, Japan, ${ }^{3}$ Department of Cancer Biology, The University of 


\section{Texas M.D. Anderson Cancer Center, USA}

Tumor blood vessels have multiple structural and functional abnormalities. Pericytes on tumor vessels are also abnormal. We have previously reported that pericytes and desmoplastic stromal cells within colon carcinoma express high levels of the PDGF-R, whereas colon cancer cells do not. We determined whether inhibition of PDGF-R tyrosine kinase signaling by imatinib would affect tumor vasculature and stromal reaction, and inhibit the growth and metastasis of human colon cancer cells growing in the subcutis (s.c.) or cecal wall of nude mice. KM12SM human colon cancer cells were injected s.c. (ectopic implantation) or into the cecal wall (orthotopic implantation) of nude mice. KM12SM cells were also injected into the spleen of nude mice to produce liver metastasis. Groups of mice $(n=10)$ received saline (control), imatinib, the cancer chemotherapeutic irinotecan, or a combination of imatinib and irinotecan. Four weeks of treatment with imatinib and irinotecan significantly inhibited tumor growth (relative to control or single-agent therapy) in the cecum and liver but not in the subcutis. In the cecum and liver, the tumors induced active stromal reaction, whereas in the subcutis, the stromal reaction was minimal. The combination therapy completely inhibited lymph node metastasis and tumor cell growth at the abdominal wall wound. Imatinib alone or in combination with irinotecan inhibited phosphorylation of PDGF-R $\beta$ of tumor-associated stromal cells and pericytes. The combination therapy also significantly decreased pericyte coverage of tumor microvessel, stromal reaction, and tumor cell proliferation, and increased the level of apoptosis in both tumor cells and tumorassociated stromal cells. These data demonstrate that the administration of a PDGF-R tyrosine kinase inhibitor in combination with irinotecan impairs the progressive growth of colon cancer cells at an orthotopic site of nude mice by blockade of PDGF-R signaling pathways in pericytes on tumor vasculature and tumor-associated stromal cells.

\section{PL 5-1}

The tumor microenvironment. The role of the cross talk between selectins and their ligands in tumor-endothelium interactions

Isaac P. Witz

Department of Cell Research \& Immunology, Tel Aviv University, Tel Aviv, Israel

The interaction between tumor and endothelial cells in the process of transendothelial migration (extravasation) is one of the pivotal steps and limiting factors of bloodborne metastasis. The transendothelial migration of leukocytes to inflammatory sites involves an interaction between selectins, chemokines and integrins with their counter receptors. The question whether or not premetastatic cancer cells employ the transendothelial migration strategy of leukocytes is being studied in our laboratory. We focus on the initial step of tumorendothelium interactions namely on the cross talk between endothelial selectins with fucosylated selectin ligands expressed by colorectal cancer (CRC) cells. This cross talk mediates the initial adhesion of CRC cells to endothelial cells. Selectin ligands are also involved in the in vivo extravasation of CRC cells. The fucose generating FX enzyme is a pivotal player in these interactions. Metastatic variants of CRC express higher levels of the FX enzyme and of fucosylated selectin ligands than variants with a lower metastatic potential. The former cells also adhere better to rE-selectin and to cytokine activated endothelial cells than the latter ones. Based on these and other results we tentatively conclude that CRC cells utilize some of the strategies employed by leukocytes to extravasate and migrate towards secondary sites to form metastasis. Since an interaction between selectins and their ligands mediates CRCendothelium communications we tested the ability of endothelial (E)-selectin to transduce signals to CRC cells and to alter gene expression patterns in these cells. rEselectin caused the phosphorylation of P38 and of ERK $1 / 2$ in CRC cells and induced a differential gene expression pattern in metastatic and non-metastatic variants. The results of these experiments will be demonstrated and discussed.

\section{PL 5-2}

\section{Tumor cell-ECM interactions: the involvement of ECM components and chemokines in tumor progression}

\section{Masayuki Miyasaka, Kazuki Sugahara}

Laboratory of Immunodynamics, Department of Microbiology and Immunology, Osaka University Graduate School of Medicine, Suita, Osaka, Japan

Tumor cells interact with a variety of extracellular matrix (ECM) components as they invade and metastasize. During tumor cell invasion, certain ECM components such as hyaluronan (HA) are often degraded into small oligosaccharides, which can be detected in patients. We previously reported that such HA oligosaccharides induce the proteolytic cleavage of an ECM-binding molecule CD44 from tumor cells and promote tumor 
cell migration in a CD44-dependent manner. Furthermore, we found that, when degraded into small oligosaccharides, chondroitin sulfate E (CSE) can also strongly enhance CD44 cleavage and tumor cell motility. In CD44-expressing tumor cells, low-molecular weight CSE (LMW-CSE) but not intact CSE enhanced CD44 cleavage; complete enzymatic digestion of LMWCSE abrogated this enhancement. Among the various sizes of LMW-CSE examined, 3-kDa CSE was the most potent inducer of CD44 cleavage. NMR analysis showed that the 3-kDa CSE bound to CD44 and that blocking this binding abrogated the CD44 cleavage induction. LMW-CSE also induced prominent motility, i.e., filopodia formation and cytoskeletal changes in tumor cells; these effects were also abrogated by blocking the LMW-CSE binding to CD44. Interestingly, CSE can avidly bind certain chemokines such as CCL21/SLC and CXCL10/IP-10, which have been implicated in lymph node metastasis of tumor cells. These results collectively indicate that LMW-CSE, which can be generated by ECM degradation, can modulate cell adhesion and migration by interacting with tumor-cell CD44 and also chemokines and suggest that LMW-CSE may play a significant role in CD44mediated tumor progression.

\section{PL 5-3}

\section{A vascular remodeling program co-opted for metastatic extravasation}

\section{Gaorav Gupta, Don X. Nguyen, Anne Chiang, Joan} Massague

\section{Memorial Sloan-Kettering Cancer Center, New York, NY, USA}

Metastasis entails numerous biological functions that collectively enable cancerous cells from a primary site to disseminate and thrive in a secondary organ. In an attempt to understand the genetic basis for this malignant cascade of events, we recently identified a gene expression signature that mediates lung metastasis in a mouse model. Significantly, we noted that a subset of these genes was also expressed by primary breast cancers from patients who later developed lung metastases, and conferred more aggressive primary tumor growth to breast cancer cells inoculated into the mouse mammary fat pad. These findings suggested that some functions necessary for metastatic progression might also promote primary tumor growth. Here we report that epiregulin, cyclooxygenase 2 , and matrix metalloproteinases- 1 and -2 constitute a multi-functional vascular re-modeling program that is expressed by aggressively lung meta- static breast cancer cells. Using genetic and pharmacological approaches, we demonstrate that this group of genes synergistically mediates blood vessel maturation in primary breast tumors, and metastatic extravasation by breaching the endothelium upon dissemination to the lungs. These mechanistic insights suggest how aggressive primary tumorigenicity can be directly linked to greater lung metastatic potential. Furthermore, the combined inhibition of this vascular re-modeling program represents a novel therapeutic strategy to impede the progression of breast cancers that express our previously described lung metastasis signature.

\section{PL 5-4}

Tumor necrosis factor and a chemokine, CCL2, contribute to liver metastasis process by acting on different liver non-parenchymal cell populations

Naofumi Mukaida, Hidekazu Kitakata, Xiaoqin Yang

Division of Molecular Bioregulation, Cancer Research Institute, Kanazawa University, Japan

The liver is populated by hepatocytes and several types of non-parenchymal cells including endothelial cells, Kupffer cells, and hepatic stellate cells. In order to clarify the precise roles of these non-parenchymal cells in liver metastasis process, we examined the liver metastasis process by using a colon adenocarcinoma cell line, colon 26.

Intrasplenic injection of colon 26 cells induced tumor necrosis factor (TNF)- $\alpha$ protein expression around the central and portal veins of the liver at 3 days and liver metastases by 24 days after the tumor injection, in $90 \%$ of wild-type (WT) mice. TNF-Rp55 KO mice developed liver metastasis at a lower rate (less than $50 \%$ ), with significantly lower liver weights and smaller volumes of metastatic foci. The intrasplenic tumor injection induced mRNA expressions of vascular endothelial growth factor, heparin-binding epidermal growth factor, matrix metalloproteinase (MMP)-9 and tissue inhibitor of matrix metalloproteinase-1 at similar levels in the livers of both WT and TNF-Rp55 KO mice. Immunohistochemical analyses of the livers of WT mice after tumor injection demonstrated the enhanced expression of vascular cell adhesion molecule (VCAM)-1 and E-selectin on sinusoidal endothelial cells. Enhancement in VCAM-1 but not E-selectin expression was significantly attenuated in the liver of TNF-Rp55 KO mice, when compared with WT mice. Thus, TNF-Rp55-mediated signals can contribute to liver metastasis by up-regulating VCAM-1 expression in the sinusoidal endothelial cells at the early step of the metastasis process. 
Intraportal injection of colon 26 cells induced macroscopic tumor focus formation in the liver 10 days after the injection. At the same time, CCL2/monocyte chemoattractant protein (MCP)-1 protein was detected predominantly in tumor cells, coincident with increased intratumoral macrophage and hepatic stellate cell numbers. In spite of a similar metastasis incidence, the number and size of metastasis foci were significantly attenuated in mice deficient in CCL2-specific receptor, CCR2, relative to wild-type mice later than 10 days after the injection. Moreover, neovascularization and MMP-2 expression were diminished in CCR2-deficient mice with a concomitant reduction in the accumulation of macrophages and hepatic stellate cells. Furthermore, matrix metalloproteinase 2 was detected predominantly in hepatic stellate cells but not in macrophages. Thus, CCR2-mediated signals can regulate the trafficking of hepatic stellate cells, a main source of MMP-2, and eventually cause neovascularization in the later step of liver metastasis process.

These observations suggest that TNF-Rp55- and CCR2-mediated signals have effects on different nonparenchymal liver cell populations at different phases of metastasis process.

\section{PL 5-5}

\section{Discovery of Aggrus/podoplanin as a metastasis- promoting platelet aggregation-inducing factor}

Naoya Fujita ${ }^{1}$, Yukinari Kato ${ }^{2}$, Takashi Tsuruo ${ }^{1}$

${ }^{1}$ The Cancer Chemotherapy Center, Japanese Foundation for Cancer Research (JFCR), Tokyo, Japan, ${ }^{2}$ Research Center for Glycoscience, National Institute of Advanced Industrial Science and Technology (AIST), Ibaraki, Japan

Specific glycoproteins expressed on the surface of platelets enable the platelets to adhere to receptors exposed in areas of vascular damage. The process of adhesion activates the platelets to aggregate, leading to the formation of a platelet plug in the vessel wall. Activated platelets also induce the formation of a fibrin clot by carrying coagulation factors and providing a catalytic surface for the major interactions of the coagulation cascade. Studies on cancer metastasis have shown that some human and animal tumor cells possess platelet aggregation-inducing abilities that correlate with their metastatic potential. Interactions between tumor cells and platelets have been considered to facilitate the arrest of tumor cell cluster in the microcirculation with the subsequent formation of experimental metastasis. However, the molecules associ- ated with the tumor-induced platelet aggregation have not yet been identified.

We have previously characterized the platelet aggregation-inducing sialoglycoprotein (Aggrus) overexpressed on the surface of tumor cells. By generating monoclonal antibodies, we revealed that the established 8F11 monoclonal antibody inhibited the platelet aggregation in vitro and the pulmonary metastasis in vivo. Using 8F11 affinity-column chromatography, we purified Aggrus protein and found that the purified protein possessed the ability to induce platelet aggregation without requiring plasma components. We recently identified that Aggrus is identical to podoplanin that is known as a lymphatic endothelial cell marker. Aggrus/podoplanin belongs to a type-I transmembrane sialomucin-like glycoprotein that consisted of an extracellular domain with abundant serine and threonine residues as potential $O$-glycosylation sites, a single transmembrane portion and a short cytoplasmic tail. Ectopic Aggrus/podoplanin expression on $\mathrm{CHO}$ cell surface induced platelet aggregation and promoted the formation of pulmonary metastasis in experimental and spontaneous metastasis models. Identification of 8F11recognition domains revealed that the highly conserved platelet aggregation-stimulating (PLAG) domains with putative $O$-glycosylated threonine residues were critical for exhibiting platelet aggregation-inducing capabilities. We compared the expression level of human aggrus mRNA using an array containing 160 cDNA pair samples derived from multiple human tumorigenic and corresponding normal tissues from individual patients. The aggrus mRNA expression is found to be frequently upregulated in seminomas and in lung squamous cell carcinomas. Immunohistochemical analysis confirmed the positive staining in sections of seminomas and lung squamous cell carcinomas. These results suggest that Aggrus/podoplanin is a newly identified platelet aggregation-inducing factor that promotes the formation of pulmonary metastasis.

\section{PL 6-1}

\section{Regulatory dendritic cells and negative regulation of immune response}

Xuetao Cao

Institute of Immunology and State Key Laboratory of Medical Immunology, Second Military Medical University, Shanghai, China

Dendritic cells (DC), the most potent professional antigen-presenting cells, can prime the immune system and 
effectively induce immune response against cancer and infectious diseases. However, DC have the ability to down-regulate immune response or induce immune tolerance. The ability of DC to initiate immune responses or induce immune tolerance depends on their maturation state and different subsets. More recently, regulatory DC with negative regulatory functions have been reported and the regulatory DC commonly used in most of current work are prepared in vitro using the immunosuppressive cytokines, such as IL-10 and TGF- $\beta$.

Here we report that: (1) Splenic stromal cells, mimics of immune microenvironment in the secondary lymphoid organs, promote the proliferation of mature DC for an extended period of time and drive them to differentiate into a new DC subset $\left(\mathrm{Ia}^{\mathrm{lo}} \mathrm{CD} 11 \mathrm{c}^{\mathrm{lo}} \mathrm{CD} 11 \mathrm{~b}{ }^{\mathrm{hi}} \mathrm{CD} 80^{\mathrm{hi}} \mathrm{CD} 40^{\text {hi }} \mathrm{CD}\right.$ $106^{\text {hi }}$ ) that potently inhibit $\mathrm{T}$ cell proliferation (Nat Immunol 2004). (2) The endothelial splenic stroma can induce hematopoietic stem cells (HSC) to differentiate into regulatory $\mathrm{CD} 11 \mathrm{~b}{ }^{\text {hi }} \mathrm{Ia}^{\text {lo }}$ DCs highly secreting TGF- $\beta$, IL-10 and NO (Blood 2006). (3) Regulatory DC display Th2biased cytokine profile by expressing high level of IL-10 but minimal IL-12 through differential regulation of ERK and p38 pathways, and secrete more IL-10 but little IL12 p70 in response to LPS or other TLR agonists including CpG ODN, LTA, and HSP70. Interestingly, regulatory DC can activate NK cells to secret IFN- $\gamma$, and these regulatory DC-activated NK cells could in turn kill surrounding regulatory DC (Blood 2006). (4) Pulmonary stroma can drive immature DC to proliferate and differentiate into distinct regulatory DC which secrete high level of TGF- $\beta$, IL-10 and PGE2, induce T cell anergy and generation of regulatory $\mathrm{T}$ cells in vitro, attenuate $\mathrm{T}$ cellmediated airway eosinophilic inflammation in vivo, suggesting that lung microenvironment may physiologically induce regulatory DC differentiation in situ, and contribute to the induction of immune homoeostasis. (5) Co-culture with tumor cells can impair the differentiation and antigen-presentation of DC, and the sorted DC from the tumor-bearing mice exhibit poor $\mathrm{T}$ cell-stimulating capacity and promote induction of regulatory $\mathrm{T}$ cells. These findings suggest that immune microenvironment and tumor microenvironment can actively induce generation of regulatory DC, forming a feedback loop that controls immune response or contributes to tumor immune escape.

\section{PL 6-2}

\section{Immature myeloid suppressor cells and dendritic cells in tumor growth and metastasis, chemoprevention, and therapy}

James E. Talmadge, Moses Donkor, Eric Scholar, Laura R. Shafer, Bela Toth, Ugur Coskun, John A.
Bishay, Traci A. Hoke

\section{University of Nebraska Medical Center, USA}

Manipulation of host immunity can control tumor growth, progression and metastasis. Conversely, tumors can suppress host immunity including tumor infiltration by histocytes. In studies of tumor and host interactions, we have observed that immature myeloid suppressor cell (IMSC) and dendritic cell (DC) numbers and tumor infiltration can be regulated by the cyclooxygenase-2 (COX-2) inhibitor, Celecoxib. Celecoxib has chemo-preventative activity for 1,2-dimethylhydrazine (1,2-DMH) induced intestinal tumors and mammary tumor development in MMTV/neu transgenic mice, significantly reducing their number and delaying their development.

The 1,2-DMH and MMTV/neu induction of tumors was associated with a significant depression in $\mathrm{CD} 3{ }^{+} \mathrm{CD} 4{ }^{+}$helper $\mathrm{T}$ cell number and significantly increased myeloid $\left(\mathrm{CD} 11 \mathrm{c}^{+} \mathrm{CD} 11 \mathrm{~b}^{+}\right)$and plasmacytoid $\left(\mathrm{CD} 11 \mathrm{c}^{+} \mathrm{B} 220^{+}\right) \mathrm{DC}$ and $\mathrm{IMSC}\left(\mathrm{CD} 11 \mathrm{~b}^{+} \mathrm{Gr} 1^{+}\right)$ numbers. Further, in association with Celebrex chemopreventive activity, these changes in IMSC and DC numbers were reversed together with a significant reduction in the tumor associated increase in nitric oxide synthase and arginase levels. These results suggest that the regulation of IMSC numbers is an important mechanism for Celecoxib chemo-preventative activity.

The growth and metastasis of the transplantable murine mammary tumors, clone-66, $4 \mathrm{~T} 1$ and MXT is associated with expansion of IMSCs, a depression in splenic T cells and tumor infiltration by IMSCs. IMSCs expansion and tumor infiltration are tumor dependent with the highly metastatic $4 \mathrm{~T} 1$ tumors resulting in the greatest increase. In all of these tumors, therapy with Celebrex can slow primary tumor growth and limit metastasis, but does not cure a significant number of mice. In addition to high levels of COX-2 mRNA, these tumors, also have high levels of mRNA for granulocytecolony stimulating factor (G-CSF) and granulocytemonocyte-CSF (GM-CSF). These growth factors, as well as fms-like tyrosine kinase ligand (Flt3L), can also expand DCs and IMSCs in mice and humans. Thus, the mechanism of IMSC expansion may not be limited solely to COX-2 levels.

We suggest that the mechanism for the increase in IMSCs and DCs in tumor-bearing animals may be due to both increased COX-2 and growth factor levels. Further, as Celecoxib administration does not increase tumor infiltration by $\mathrm{T}$ cells or decrease IMSC infiltration, growth factor inhibition may also be required to further improve clinical outcomes. 
Together, these studies, suggest that we need to consider as part of host tumor interactions, the role of immune augmentation and tumor suppression of immunity. Supported in part by funding from NIH grant 5 RO1 AT 001739, Eppley Cancer Center and Nebraska DHHS LB 506.

\section{PL 6-3}

\section{Notch 2 signaling controls cytotoxic $T$ cell differentiation}

\section{Koji Yasutomo}

Department of Immunology \& Parasitology, IHBS, The University of Tokushima, Tokushima, Japan

Notch signaling generally controls cell fate choices. The mammal possesses four Notch molecules and five Notch ligands. Recent studies have revealed that Notch signaling regulates $\mathrm{T}$ cell effector functions including helper $\mathrm{T}$ cell differentiation as well as $\mathrm{T}$ cell development from lymphoid progenitors. We here examined whether Notch signaling regulates differentiation of cytotoxic $\mathrm{T}$ cells from naive $\mathrm{CD} 8+\mathrm{T}$ cells. The $\mathrm{CD} 8+\mathrm{T}$ cells begin to express Notch2 after $\mathrm{T}$ cell receptor ligation. The interaction between Delta1, one of the Notch ligands expressed on dendritic cells, and Notch2 enhanced the proliferation and killing activity of $\mathrm{CD} 8+\mathrm{T}$ cells. The CD8 $+\mathrm{T}$ cells from Notch2 deficient mouse had a defect in generating cytotoxic $\mathrm{T}$ cells. Furthermore, the downregulation of Deltal on dendritic cells significantly reduced the expansion of cytotoxic $\mathrm{T}$ cells. The Notch 2 signaling directly controlled the transcription of granzyme B that is a main effector molecule for cytotoxicity. The inoculation of dendritic cells overexpressing Delta1 around tumors inhibited the evasion of tumors by expanding cytotoxic $\mathrm{T}$ cells. Those results indicate that Notch2 signaling is a physiologically critical pathway to expand cytotoxic $\mathrm{T}$ cells. The modification of this pathway may be a good target to enhance tumor immunity.

\section{PL 6-4}

\section{Non-immunogenic tumor antigen-based vaccines targeted to APCs induce therapeutic antitumor immunity}

Arya Biragyn, Dolgor Baatar, Purevdorj Olkhanud

National Institute on Aging, NIH, USA

We have recently established a simple strategy for rendering weakly or non-immunogenic self-tumor antigens immunogenic by delivering them to chemokine receptors expressed on APCs [1, 2]. The breadth of the strategy was in the ability of chemokines to efficiently utilize chemokine receptor-mediated uptake and the MHC class I and II processing and presentation pathways [3, 4]. As result, DNA immunizations with chemokine-based vaccines encoding the embryonic antigen OFA-iLRP, expressed by a wide variety of tumors, elicited $\mathrm{CD} 8+\mathrm{T}$ cell-mediated therapeutic antitumor immunity against syngeneic murine tumors. Furthermore, the vaccine efficacy was improved by local depletion of CCR4+ cells, presumably Tregs. Specific killing of CCR $4+$ cells completely reversed a suppressive state of $\mathrm{T}$ cells and significantly augmented vaccine-induced $\mathrm{CD} 8 \mathrm{~T}$ cell and antitumor responses. Thus, these data indicate that potent antitumor responses can be elicited when vaccines are combined with the local and transient depletion of immunosuppressive cells.

Acknowledgements This research was supported by the Intramural Research Program of the NIH, National Institute on Aging.

\section{References}

1. Biragyn A et al (1999) Genetic fusion of chemokines to a self tumor antigen induces protective, T-cell dependent antitumor immunity. Nat Biotechnol 17(3):253-258

2. Biragyn A et al (2001) Mediators of innate immunity that target immature, but not mature, dendritic cells induce antitumor immunity when genetically fused with nonimmunogenic tumor antigens. J Immunol 167:6644-6653

3. Biragyn A et al (2004) Chemokine receptor-mediated delivery directs self-tumor antigen efficiently into the class II processing pathway in vitro and induces protective immunity in vivo. Blood 104(7):1961-1969

4. Schiavo et al (2006) Chemokine receptor targeting efficiently directs antigens to MHC class I pathways and elicits antigen-specific $\mathrm{CD} 8+$ Tcell responses. Blood 107(12):4597-4605

\section{PL7-1}

On the role of galectin-3 in tumor metastasis from the bench to the clinic and back

Avraham Raz

Wayne State University, USA 
Galectin-3, a member of the $\beta$-galactoside-binding gene family, is a multifunctional protein implicated in a variety of biological functions, including tumor cell adhesion, proliferation, differentiation, angiogenesis, cancer progression and metastasis. Recent studies revealed that intracellular galectin-3 exhibits the activity to suppress drug induced apoptosis and anoikis (apoptosis induced by the loss of cell anchorage) that contribute to cell survival. Resistance to apoptosis is essential for cancer cell survival and plays a role in tumor progression. Conversely, it was recently shown that tumor cells' secreted galectin-3 induces T-cells' apoptosis, thus playing a role in the immune escape mechanism during tumor progression through induction of apoptosis of cancer infiltrating T-cells. I'll summarize recent evidences on the role of galectin-3 as an anti-apoptotic and/or pro-apoptotic factor in various cell types and discusses the recent understanding of the molecular mechanisms of galectin-3 role in apoptosis. I'll also discuss potential therapeutic directions for further analyses of this multifunctional protein.

\section{PL7-2}

\section{MT1-MMP: a potent regulator of cancer cell behavior}

\section{Motoharu Seiki}

Div. Cancer Cell Research, Institute of Medical Science, University of Tokyo, Tokyo, Japan

Remodeling of tissue requires one or combinations of multiple matrix metalloproteinases (MMPs) depending on the components of extracellular matrix (ECM) in tissue. Mammals have 23 MMPs and six of them are membrane-anchored enzymes (MT-MMPs) having either a transmembrane domain or an anchor signal for GPI at the C-terminus. Since MT-MMPs are expressed on the cell surface, they are expected to play roles in pericellular proteolysis at the cell-ECM and cell-cell interface.

MT1-MMP is the firstly identified member of MTMMPs and the most extensively studied one. It was demonstrated to act as a cell-mediated activator of MMP-2 which is believed to be critical for tumor cells to traverse the basement membrane by degrading type IV collagen. Since MT1-MMP is expressed in human tumors and correlates with activation of MMP-2 in the tissue, it was proposed to be a key player for tumor invasion by activating MMP-2.

While the intriguing cell-mediated mechanism of MMP-2 activation by MT1-MMP has been studied extensively, a variety of potential substrates of MT1-
MMP have been identified. These are various ECM components such as collagen I, membrane proteins including cell adhesion molecules, and soluble bioactive proteins such as cytokines. However, physiological significance of the cleavage of these substrates has not been necessarily clarified for each substrate in vivo. Recently importance of MT1-MMP as a cell associated collagenase has been particularly emphasized both in vitro and in vivo. Since collagen $I$ is the most abundant ECM components in tissue, interaction of cells with collagen I is particularly important for regulation of cell functions. Its importance as a collagenase was clearly demonstrated using MT1-MMP deficient mice. The mice die within 2-3 weeks after birth with aberrant bone formation, and severe fibrosis as a result of defect in collagenolysis. These results suggest that cell-mediated collagenolysis by MT1-MMP is hardly substitutable with other proteases. The collagenase activity of MT1MMP has been demonstrated to be critical and indispensable for tumor cell invasion and proliferation in collagen-rich environment.

In my presentation I will show how collagenase activity of MT1-MMP and MMP-2 activated by MT1MMP co-operate to promote tumor growth in vitro and in vivo. MT1-MMP is also a critical component in angiogenesis expressed in the endothelial cells and a potential therapeutic target. I will also address how MT1-MMP expression and function is regulated during angiogenesis through the crosstalk between endothelial cells and pericytes.

\section{PL7-3}

\section{The nucleoside diphosphate kinase \\ and $3^{\prime}-\mathbf{5}^{\prime}$ exonuclease activities of NM 23-H1 may contribute to suppression of metastasis in melanoma cells: a complementation approach}

David M. Kaetzela, Qingbei Zhang, Joseph R. McCorkle, Mengmeng Yang

Department of Molecular and Biomedical Pharmacology, College of Medicine, University of Kentucky, USA

NM23-H1 exhibits three enzymatic activities in vitro that may contribute its metastasis suppressor activity. The protein contains a catalytic histidine residue (H118) through which it transfers a phosphate moiety to either nucleoside diphosphate (nucleoside diphosphate kinase, or NDPK) or protein substrates (histidine kinase, or hisK). In addition, it harbors a $3^{\prime}-5^{\prime}$ exonuclease (EXO) activity when presented with DNA containing nonduplexed, 3'-terminal nucleotides, suggestive of roles in DNA repair, replication, and/or recombination (Ma et al. 
2004). Previous studies have shown that a hisK-deficient variant of NM23-H1 (P96S) in cultured breast carcinoma (MDA-MB-231) fails to exhibit the antimotility effect of the wild-type protein, indicating a potential antimetastatic function for the hisK (MacDonald et al. 1996). In the current study, a similar complementation approach was undertaken to compare the relative roles of all three enzymatic activities in mediating the metastasis suppressor actions of NM23-H1. The metastastic melanoma cell line $1205 \mathrm{Lu}$ was chosen as an experimental model by virtue of its low endogenous NM23 expression, as well as the ability of NM23-H1 to suppress its metastatic phenotype in culture and in vivo. To compare the relative contributions of each enzymatic activity, NM23-H1 variants were identified that harbor lesions in one or more of the three activities, and their forced expression achieved by stable transfection. The mutant forms consisted of the hisK-deficient mutant P96S, as well as H118F (NDPK- and hisK-deficient), and K12Q, which is deficient in all three enzymatic activities (Ma et al. 2004). With respect to metastatic characteristics of these cells in culture, none of the three mutants suppressed motility or invasive capacity to the extent shown by the wild-type protein, strongly suggesting a role for the hisK in these indices of metastatic growth. When these cell lines were analyzed in athymic nude mice for their ability to metastasize to lung from primary tumor explants (i.e., spontaneous metastasis), a different hierarchy was observed, with the H118F and K12Q but not the P96S exhibiting a significant loss in suppressor activity. In light of potential roles of both the EXO and possibly NDPK activity in genetic progression to the metastatic phenotype, the panel of wild-type and mutant NM23-H1 variants were also expressed in the melanoma cell line WM793, which are non-metastatic but can progress to a metastatic phenotype when subjected to selective pressure (Kath et al. 1991). Interestingly, forced expression of wild-type NM23-H1 led to inhibition of progression to growth-factor-independence in culture, consistent with a novel anti-progression activity. Similar to the pattern observed in $1205 \mathrm{Lu}$ cells, H118F and K12Q both lost this ability while the hisK-deficient mutant P96S exhibited unimpaired activity. Taken together, our studies indicate that the hisK activity appears to suppress motility and invasion while the NDPK, and possibly the EXO, appear to suppress progression to metastasis in melanoma.

\section{PL 7-4}

\footnotetext{
ADAM17-mediated CD44 ectodomain cleavage plays a critical role in hyaluronic acid turnover and cancer cell migration in extracellular matrix
}

Hideyuki Saya, Osamu Nagano

Department of Tumor Genetics and Biology, Graduate School of Medical Sciences, Kumamoto University, Kumamoto, Japan

There are multiple steps in the metastasis of cancer cells. Tumor-cells must first be dissociated from the tumor mass and invade into the surrounding extracellular matrix (ECM). In these processes, cell surface adhesion molecules play an important role in the interaction between the cells and their microenvironments. CD44 is an adhesion molecule that interacts with hyaluronic acid (HA) and implicated in a wide variety of physiological and pathological processes. Recently, the proteolytic cleavage of CD44 has been emerging as key regulatory events for the CD44 dependent cell-matrix interaction and signaling pathway. CD44 undergoes sequential proteolytic cleavage in the ectodomain and intramembranous domain, resulting in the release of a CD44 intracellular domain (ICD) fragment. The ectodomain cleavage of CD44 is triggered by multiple stimulations and contributes to the regulation of cell attachment to and migration on HA matrix. The ectodomain cleavage subsequently induces the intramembranous cleavage which is mediated by presenilin (PS)-dependent $\gamma$-secretase. The intramembranous cleavage generates CD44 ICD, which acts as a signal transduction molecule, where it translocates to the nucleus and activates transcription. The ectodomain cleavage of CD44 is triggered by extracellular $\mathrm{Ca}^{2+}$ influx or the activation of protein kinase $\mathrm{C}$ (PKC) and is mediated by two different ADAM family metalloproteases, ADAM10 or ADAM17, respectively. Depletion of those ADAM proteases by RNAi effectively blocks cancer cells to invade in HA matrix. Especially, downregulation of ADAM17 induces the accumulation of intracellular HA and CD44 as well as the increase in pericellular HA matrix. Furthermore, we found that phosphorylation of moesin by PKC is a crucial step for triggering the endocytosis of CD44-HA complex, and following CD44 ectodomain cleavage. In the absence of ADAM17-mediated CD44 cleavage, intracellular CD44HA is stabilized and accumulated in the Rab11-positive recycling endosomal compartments. These findings suggest that PKC/ADAM17-mediated CD44 ectodomain cleavage plays an important role in engulfment and turnover of HA, facilitating ECM remodeling which activates cancer cell motility in the HA-rich ECM.

\section{PL 7-5}

The NCAM/FGFR signaling complex: a novel player in metastatic dissemination 
Ugo Cavallaro $^{1}$, Chiara Francavilla ${ }^{1}$, Sebastien

Loeffler $^{1}$, Gerhard Christofori ${ }^{2}$

${ }^{1}$ IFOM-FIRC Institute of Molecular Oncology, Milan, Italy, ${ }^{2}$ Institute of Biochemistry and Genetics, University of Basel, Switzerland

The neural cell adhesion molecule (NCAM) has long been known to modulate cell-cell interactions and neuronal migration in the nervous system. In addition, NCAM is expressed in other cell types outside the nervous system, where its function has remained largely elusive. Importantly, the expression of NCAM is downregulated in a subset of human tumors, and this downregulation correlates with poor prognosis.

To address the function of NCAM in tumor progression, we have abrogated its expression in a transgenic mouse model of multistage carcinogenesis. The loss of NCAM was sufficient to induce lymph node metastasis, indicating that NCAM acts a metastasissuppressing factor. Interestingly, by analogy to certain human cancer types, the metastatic lesions in NCAMdeficient mice retained a benign phenotype, thus challenging the general view of metastatic dissemination as a process restricted to frankly malignant cancer cells. Moreover, NCAM-deficient tumors showed a dramatic defect in tumor-microenvironment interactions. Indeed, we observed that the loss of NCAM results in an impaired beta 1 integrin-dependent cell-matrix adhesion. We could then demonstrate that NCAM controls tumor cell-matrix adhesion by associating with the fibroblast growth factor receptor (FGFR) and stimulating FGFR activity. More recently, we have dissected the signaling pathways downstream of the NCAM/FGFR complex and found that NCAM acts as a bona fide ligand for FGFR, triggering a signaling cascade that is distinct from that induced by the classical FGFR ligand, FGF. Finally, our results have revealed an unexpected role for NCAM in modulating the cellular response to FGF.

Overall, these observations highlighted novel properties of NCAM as an anti-metastatic factor and as a molecular switch for FGFR. Based on studies in patients, these new functions of NCAM are likely to have important implications for the metastatic potential and, hence, the prognosis of certain human tumor types.

\section{PL 7-6}

\section{Roles of membrane-type matrix metalloproteinase-1 in tumor invasion and metastasis}

Hiroshi Sato, Takahisa Takino, Hisashi Miyamori

Department of Molecular Virology and Oncology,
Cancer Research Institute, Kanazawa University, Japan

Matrix metalloproteinases (MMPs) are involved in tumor invasion and metastasis. The invading cells are forced to proliferate within embedded dense threedimensional matrix composed largely of type I collagen. Membrane-type MMP-1 (MT1-MMP) has been thought to play a major role in this step. Collagen matrix acts as physical barrier for tumor-cell invasion, but at the same time it provides cells with various signals for growth, survival, differentiation, migration and so on. Extra-cellular signal-regulated kinase (ERK) is activated in cells cultured in Type I collagen matrix or on collagen sheet. ERK activated by collagen culture of cells in turn stimulates MT1-MMP expression. MT1-MMP thus induced by ERK plays an essential role in sustained activation of ERK. Activation of ERK is not maintained in the presence of MT1-MMP inhibitor. Thus, MT1-MMP functions in a positive feedback loop to induce sustained ERK activation and subsequent MT1-MMP accumulation, which collectively promotes cell migration on collagen.

ECM regulates cell growth/survival and differentiation, which vary between tissues. Some of ECM components such as decorin and lumican, both of which belong to a small lecine-rich proteoglycan family negatively regulate cell growth, and are classified as tumor suppressors. Both docorin and lumican were shown to induce p21/Waf-1 an inhibitor of cyclin-dependent kinases in tumor cells, and down-regulates cell growth and tumorigenicity. MT1-MMP expressed on the surface of tumor cells degrades decorin and lumican, which restores tumorigenicity.

Various substrates for MT1-MMP other than ECM components have been discovered such as adherence molecules, cytokines/growth factors and cell surface receptors. While the physiological significance of these activities of MT1-MMP still remains to be explored, MT1-MMP appears to be involved in regulation of various events taking place at the cell-ECM interface.

These multi-functions of MT1-MMP may play important roles in not only initial step of tumor invasion but also the cell growth in distant organs where ECM environments are different from that of original tissue.

\section{PL 7-7}

Enhanced expression of the cathepsin inhibitor Stefin $A$ is associated with metastasis in breast cancer

Belinda S. Parker ${ }^{1}$, Bradley N. Bidwell ${ }^{1,2}$, John L. Slavin $^{3,4}$, Normand Pouliot ${ }^{1}$, Michael A. Henderson ${ }^{5}$, Robin L. Anderson ${ }^{1,3}$ 
${ }^{1}$ Department of Research, Peter MacCallum Cancer Centre, East Melbourne, Australia, ${ }^{2}$ Department of Biochemistry and Molecular Biology, University of Melbourne, Parkville, Australia, ${ }^{3}$ Department of Pathology, University of Melbourne, Parkville, Australia, ${ }^{4}$ Department of Pathology, St Vincents Hospital, Fitzroy, Australia, ${ }^{5}$ Division of Surgical Oncology, Peter MacCallum Cancer Centre, East Melbourne, Australia

Metastatic breast cancer is a life threatening disease with limited treatment options. Molecular markers that can either predict accurately if a patient is likely to develop metastatic disease or be used as therapeutic targets are lacking. To address this, we have used a clinically relevant murine model of spontaneous metastasis to lung and bone, to identify candidate genes involved in metastatic progression. By microarray profiling and immunohistochemistry, we found enhanced expression of the cathepsin inhibitor Stefin A in epithelial cells isolated from primary tumors and their matched spontaneous metastases in lung and bone. In the human disease, patients lacking primary tumor expression of Stefin A had improved disease free survival and expression of Stefin A was enhanced in lung and bone metastases. As an endogenous inhibitor, we proposed that Stefin A is a marker of enhanced cathepsin activity in metastatic tumors. To explore this, we measured cathepsin activity using protease specific fluorescent substrates and found increased activity of cathepsin B in highly metastatic primary tumors. Stefin A and cathepsin B co-localise at the invasive fronts of primary tumors and in lung and bone metastases in the murine model and in human breast cancer. This study implicates cathepsins and their inhibitor Stefin A in breast cancer metastasis and provides evidence that Stefin A has prognostic value in breast cancer.

\section{PL 7-8}

\section{SSX-new molecular target regulating cancer cell motility and invasion}

Kazuyuki Itoh ${ }^{1}$, Norifumi Naka ${ }^{2}$, Kiyoko Yoshioka ${ }^{1}$

${ }^{1}$ Department of Biology, Osaka Medical Center for Cancer and Cardiovascular Diseases, Osaka, Japan, ${ }^{2}$ Department of Orthopedic Surgery, Osaka Medical Center for Cancer and Cardiovascular Diseases, Osaka, Japan

Cell migration is mainly driven by actin-based cytoskeletal reorganization and myosin-based motor activity, both regulated with Rho-family small GTPases. Since the main regulatory mechanism is common for both cancer cells and normal cells, such as lymphocytes, seeking the cancer-specific molecule, which regulates cancer cell motility, is required for better understanding of cancer invasion and future target therapy. The SSX genes were initially identified as fusion partners to the SYT gene in human synovial sarcomas carrying a recurrent $\mathrm{t}(\mathrm{X} ; 18)(\mathrm{p} 11.2 ; \mathrm{q} 11.2)$ chromosomal translocation. Besides adult human testis, SSX genes were expressed at varying frequencies in a number of malignancies thereby categorized as cancer/testis antigens. Using Nucleic Acid SequenceBased Amplification, we have reported that the expression level in the malignant tumors $(3.8 \pm 1.4$ copies $/ \mu \mathrm{g}$ of total RNA in $\log 10$ order) was significantly higher than that in the benign tumors (2.4 $\pm 0.5, \quad P<0.0001)$. In order to examine the biological function of SSX, we firstly made stable transfectants with wild type SSX using human osteosarcoma cell line, Saos-2, which moderately expressed SSX. The expression of SSX was mainly localized in the nucleus with patched pattern. The SSX transfectants promoted colony formation in soft agar and tumor formation in nude mice, but showed little change in growth rate in $2 \mathrm{D}$ culture. The transfectants also increased motility, chemotaxis and invasiveness using scratch wound assay and Boyden chamber assay. Enhanced Rho activity and stress fibers were observed in these SSX overexpressed Saos-2 cells. By contrast, the lowering of the endogenous expression of SSX 1 in fibrosarcoma HT1080 cells by the treatment with specific siRNA markedly decreased membrane ruffling, chemotaxis and invasiveness using Boyden chamber assay, but did not affect cell proliferation in 2D culture. Moreover, SSX1 deficient HT1080 cells showed decreased Racl-activation and myosin light chain phosphorylation. Collectively, these data suggested that SSX protein regulated Rho-family small GTPases leading to the tumor invasion and progression, and thus could be a new molecular target under clinical setting.

\section{PL 7-9}

\section{Tumor hypoxia induces expression of carbohydrate determinants involved in adhesion of circulating cancer cells to vascular endothelium}

Reiji Kannagi

Department of Molecular Pathology, Aichi Cancer Center, Nagoya, Japan 
Cell adhesion mediated by selectins and their carbohydrate ligands, sialyl Lewis X and sialyl Lewis A, plays an important role in cancer metastasis and tumor angiogenesis. Expression of these carbohydrate determinants is markedly enhanced in cancer cells compared to non-malignant epithelial cells $[1,2]$. Our recent studies indicated the presence of further-modified complex forms of sialyl Lewis $\mathrm{X}$ and sialyl Lewis $\mathrm{A}$ in non-malignant epithelial cells, which have additional sulfation or sialylation. The impairment of these additional modifications in cancer cells, which we call "incomplete synthesis," leads to a considerable accumulation of sialyl Lewis X/A in cancer cells at early stages of malignant transformation [3]. Epigenetic changes such as DNA methylation and/or histone deacetylation confer the incomplete synthesis. In later stages of cancer progression, the increased transcription of several genes responsible for the synthesis of sialyl Lewis X/A determinants further accelerates expression of these determinants on cancer cells. We recently showed that these changes are at least partly mediated by sustained expression of transcription factors called hypoxia inducible factors (HIFs) in cancer cells, which induce transcription of the genes for a fucosyltransferase (FUT7), a sialyltransferase (ST3O), UDPgalactose transporter-1 (UGT1) and GLUT-1, all of which are intimately involved in cellular sialyl Lewis $\mathrm{X} / \mathrm{A}$ synthesis [4]. Cancer cells in hypoxic environment in the locally-advanced tumors accumulate genetic abnormalities, and more malignant hypoxia-resistant cancer cell clones evolve according to the principle of survival of the fittest. These hypoxia-resistant cancer cells are known to have higher infiltrative and metastatic activities. Expression of sialyl Lewis X/A determinants is further accelerated during the course of cancer progression. Tumor hypoxia also induces expression several other genes involved in cell surface carbohydrate synthesis, which leads to extensive modification of carbohydrate determinants on cancer cells. A good example is hypoxia-induced expression of $N$-glycolyl GM2, a carbohydrate determinant carrying unique $N$-glycolyl sialic acid [5]. Its expression is induced by enhanced transcription of the gene for sialin, a sialic acid transporter. As hypoxia is associated with diminished response to radio- and chemotherapy, $N$-glycolyl GM2 is a potential therapeutic target for hypoxic cancer cells.

\section{PL 8-1}

\section{Nm23-H1 suppresses tumor cell motility through the downregulation of the LPA receptor EDG2 and the hepatocyte growth factor receptor MET}

Patricia S. Steeg ${ }^{1}$, Jong Heun Lee ${ }^{1}$, Abdel Elkahloun ${ }^{2}$,
Mathieu Boissan ${ }^{3}$, Sylvie Dumont ${ }^{3}$,Tara K. Maga ${ }^{1}$, James G. Christensen ${ }^{4}$, Sandrine Arnaud-Dabernat ${ }^{5}$, Diane Palmieri ${ }^{1}$, Jean-Yves Daniel ${ }^{5}$, Marie-Lise Lacombe $^{3}$, Paul S. Meltzer ${ }^{2}$, Christine E. Horak ${ }^{1}$

${ }^{1}$ National Cancer Institute, ${ }^{2}$ National Human Genome Research Institute, ${ }^{3}$ Institut National de la Sante et de la Recherche Medicale, ${ }^{4}$ Pfizer Global Research and Development, ${ }^{5}$ Universite Victor Segalen Bordeaux

Diminished expression of the metastasis suppressor Nm23-H1 significantly correlates with tumor aggressiveness in breast cancer. Exogenous overexpression of $\mathrm{Nm} 23-\mathrm{H} 1$ in the metastatic breast carcinoma cell line MDA-MB-435 reduces the metastatic capacity of these cells as determined by in vivo mouse injection experiments and in vitro assays of cell motility and soft agar colonization. Mutational analysis of Nm23$\mathrm{H} 1$ has revealed that substitution mutants P96S and S120G are unable to inhibit cell motility and colonization. To elucidate the molecular mechanism by which Nm23H1 mediates motility suppression, we performed microarray expression analysis using a comprehensive oligonucleotide array and mRNA extracted from MDA-MB-435 cells stably overexpressing wild-type Nm23-H1 and the P96S and S120G mutants as compared to a control-transfected cell line. Approximately 70 genes are specifically regulated by wild-type Nm23-H1, but not by the mutants. Presumably, these genes are critical to metastatic suppression. Using quantitative RT-PCR and immunoblot analysis, the observed patterns of microarray expression for several of these genes have been confirmed in both MDA-MB-435 and MDA-MB231 breast carcinoma cell lines stably-transfected with Nm23-H1. The clinical relevance of the relationship between $\mathrm{Nm} 23-\mathrm{H} 1$ and its regulated genes has been determined through analysis of published microarray data of two breast tumor cohorts. Within these datasets, a significant correlation has been established between expression of the Nm23-H1 gene and expression of 19 genes that it regulates, including $M E T, P T N, L 1 C A M$ and EDG2. The protein levels of several of these genes were examined by immunohistochemistry (IHC) in tumor tissues from the recently engineered NM23-MI knockout mouse, which is prone to lung metastasis when induced to develop hepatocellular carcinoma (HCC). HCC of the NM23M1-/- mouse had much higher levels of EDG2 and MET protein compared to HCC from wild-type mice supporting the hypothesis that Nm23-H1 downregulates these two genes in vivo. Finally, we have established a functional connection between Nm23-H1 
regulated genes and suppression of cell motility, an important in vitro hallmark of metastasis. Of nine genes tested, only overexpression of EDG2 and MET restored the motility phenotype to Nm23-H1-expressing MDA-MB-435 cells by 60- and 4-fold, respectively. $E D G 2$ encodes for a G-protein coupled receptor that binds to LPA, the lipid mediator that is a significant component of serum and regulates cell proliferation, differentiation and motility and MET encodes for the hepatocyte growth factor receptor. $\mathrm{Nm} 23-\mathrm{H} 1$ suppresses motility, at least in part, through the coordinated down-regulation of the EDG2 and $M E T$ genes.

\section{PL 8-2}

\section{When do lung cancer cells become invasive and metastatic and what genes are responsible for these phenotypic alterations of lung cancer cells?}

Jun Yokota, Kenji Takahashi, Shingo Matsumoto, Reika Iwakawa, Takashi Kohno

\section{Biology Division, National Cancer Center Research} Institute, Tokyo, Japan

It is now widely accepted that cancer is caused by accumulation of genetic alterations in somatic cells. However, it is still unknown when cancer cells acquire invasiveness and metastatic ability, and what genes are responsible for these phenotypic alterations of cancer cells. To understand the molecular processes of lung cancer progression and to elucidate genes responsible for acquisition of invasiveness and metastatic ability in lung cancer cells, comprehensive and comparative studies on genetic alterations in noninvasive/invasive as well as primary/metastatic lung carcinomas are in progress in our laboratory. The results of molecular analyses on small adenocarcinomas as well as those on primary and metastatic lung carcinomas of the same patients will be presented, and possible molecular processes of lung cancer progression will be discussed.

To understand the molecular process of early stage lung adenocarcinoma progression, accumulated genetic alterations were compared between non-invasive and invasive small adenocarcinoma of the lung $(>2 \mathrm{~cm}$ in diameter). All p53 mutations were detected in invasive carcinoma, whereas EGFR and KRAS mutations were detected in both invasive and non-invasive carcinoma. Thus, it was indicated that EGFR and KRAS mutations are two of critical genetic alterations responsible for the formation of non-invasive adenocarcinoma while p53 mutations are associated with phenotypic alterations of adenocarcinoma from non-invasive to invasive ones.

Several models of cancer progression, including the clonal evolution, parallel evolution and same-gene models, have been proposed to date. To investigate the authenticity of these models, accumulated genetic alterations were compared between primary and corresponding metastatic lung cancers. A whole genome allelic imbalance (AI) scanning using a high-resolution SNP array and mutational analysis of the p53, EGFR and KRAS gene were also performed on eight sets of primary and metastatic lung cancers. Five to twenty chromosomal regions showed $\mathrm{AI}$ in each tumor. Accumulated genetic alterations were similar between primary and corresponding metastatic tumors, and the majority $(>67 \%)$ of genetic alterations detected in metastatic tumors were also detected in the corresponding primary tumors. On the other hand, in seven of the eight cases, there were genetic alterations accumulated only in metastatic tumors. Likewise, four cases showed genetic alterations detected only in primary tumors. The natural history of each case indicated that the process of metastasis varies among cases and that all three models are applicable to lung cancer progression.

\section{PL 8-3}

\section{Signalling pathways as targets for therapy in angiogenesis and metastasis}

\section{Suzanne A. Eccles}

\section{Institute of Cancer Research, UK}

Key determinants of tumour progression are neoangiogenesis and the capacity to invade and metastasise. Cell motility, chemokinesis and deregulated proteolysis are implicated in both tumour cell invasion and the response of activated endothelial cells to angiogenic cytokines. It is increasingly recognised that these processes might provide a rich source of novel targets for cancer therapy and that appropriate inhibitors may restrain both metastasis and neoangiogenesis with fewer side effects than classical cytotoxic therapies.

Oncogenic tyrosine kinase receptors (e.g. EGFR, the ErbB family, MET etc.) and angiogenic cytokine receptors on endothelial cells activate key signalling cascades including PI3 kinase, PLC $\gamma$ and MAP kinases which collectively contribute to cell survival, proliferation, migration and differentiation. We are exploring the potential of inhibitors of these pathways for therapeutic benefit against metastasis, and will report on their efficacy in preclinical models. 
PI3 kinase antagonists inhibited in vitro chemomigration and haptotaxis of tumour cells in vitro and also downregulated specific matrix metalloproteinases induced by EGFR activation. Novel inhibitors have also shown activity in several human tumour xenografts, with clear downregulation of pharmacodynamic markers of response. Inhibition of the PI3-kinase pathway prevents induction of VEGF via activated oncogenes and/or hypoxia. Also, human endothelial cell (EC) proliferation, migration, differentiation into tubules in vitro and tumour angiogenesis in vivo was inhibited.

Secondly, we showed that PLC $\gamma$ plays a major role in tumour cell and endothelial cell motogenic responses to both activated receptor tyrosine kinases and $\beta 1$ integrins. We are now developing inhibitors of this potential new therapeutic target, and will examine effects of disabling both PLC $\gamma$ and PI3k pathways since there is evidence of compensatory activation.

Heat shock protein 90 (Hsp90) chaperones several key molecules including MET, ErbB-2, AKT and RAF proteins. Inhibitors such as $17 \mathrm{AAG}$ can thus effectively and simultaneously disrupt several parallel signalling pathways. 17AAG downregulated expression of client proteins in human tumour cells and EC in a concentration- and time-dependent manner and inhibited haptotaxis and chemomigration towards a variety of ligands. Invasion was more potently inhibited than migration, and this was linked to inhibition of uPA production. In vivo we found that murine endothelial cell client proteins (including VEGF receptors) were down-regulated by 17AAG and inhibition of growth and metastasis of human tumour xenografts was associated with reduced microvessel density.

Future work will identify optimal combinations of novel inhibitors for the prevention and treatment of disseminated disease.

\section{PL 8-4}

\section{Attenuation of anti-metastasis defense in target organs by cancer-derived cytokine}

Hiroki Kuniyasu

Department of Molecular Pathology, Nara Medical University, Kashihara, Japan

A balance between cancer and anti-metastasis defense in the metastasis-target organs is a key factor in the process of metastasis formation. In this presentation, a mechanism of reduction in anti-metastasis defense in the lymph nodes and liver by HMGB1 secreted from colon cancer cells will be introduced. HMGB1 is a bifunc- tional protein; a chromatin component regulating transcriptional gene expression and DNA repair, and an inflammatory cytokine worsening endotoxic shock by activation of IL-1, TNF- $\alpha$, and IFN- $\gamma$. HMGB1 is released from nuclei under chromatin histone acetylation or cell necrosis. In cancer cells, HMGB1 activates its receptor, RAGE (receptor for advanced glycation end products), which is a multifunctional membrane protein, to accelerate proliferation, motility, invasion, and angiogenesis. Concurrent expression of HMGB1 and RAGE is found in all colon cancers with distant metastasis to the liver or lung. The colon cancer cases with concurrent expression of HMGB1 and RAGE show poorer prognoses than those without concurrent expression. In macrophages, HMGB1 induces apoptosis at high concentration via JNK phosphorylation. HMGB1-induced apoptosis inhibits macrophage infiltration into the tumors. HMGB1 also affects resident macrophages in the lymph nodes and liver. HMGB1 treatment reduces the number of nodal sinus macrophages and hepatic Kupffer cells in mice. HMGB1 concentration in non-metastasized lymph nodes in human colon cancer with nodal metastasis is higher than that in cancer without metastasis, whereas the number of sinus macrophages is lower than that in non-metastasized cancers. Pretreatment with HMGB1 administrated into the cecum wall decreases the number of Kupffer cells and increases the number of embedded colon cancer cells in the liver, which were injected into the spleen. In human cases, sera obtained from portal brunches in the mesocolon show higher HMGB1 concentration in liver-metastasis cases than that in no metastasis cases. These findings suggest that HMGB1 secreted from colon cancer cells in the primary tumors reaches the metastasis-target organs via portal or lymphatic flow, and suppresses the resident macrophages to reduce resistance on cancer cell embedding. The "priming" effect of cancer-derived cytokines on the metastasis-target organs might be a pivotal scenario in cancer metastasis.

\section{PL 8-5}

\section{Gene regulation in melanoma progression:} implications for therapy

Menashe Bar-Eli

The University of Texas, M.D. Anderson Cancer Center, USA

The molecular changes associated with the transition of melanoma cells from radial growth phase (RGP) to vertical growth phase (VGP, metastatic phenotype) 
are not yet well defined. We have demonstrated that the progression of human melanoma is associated with loss of expression of the transcription factor AP2. In metastatic melanoma cells, this loss resulted in overexpression of MCAM/MUC18 and MMP2 and lack of c-KIT expression. In addition, inactivation of AP-2 in primary cutaneous melanoma cells by dominant negative AP-2 (AP-2 B) augmented their tumorigenicity in nude mice. We have also recently demonstrated that loss of AP-2 expression in metastatic melanoma cells resulted in over production of the thrombin receptor, PAR-1, which in turn, contributes to the metastatic phenotype of melanoma by upregulating the expression of adhesion molecules, proteases, and angiogenic factors. Additionally, the transition of melanoma cells from RGP to VGP is associated with overexpression of the transcription factors CREB and ATF1, both of which may act as survival factors for human melanoma cells. Inactivation of CREB/ATF-1 activities in metastatic melanoma cells by dominant-negative CREB or by antiATF1 single chain antibody fragment (ScFv), resulted in deregulation of MMP-2 and MCAM/MUC18, increase the sensitivity of melanoma cells to apoptosis, and inhibition of their tumorigenicity and metastatic potential in vivo. In recent studies we have identified the platelet activating factor (PAF) which is secreted by cells within the tumor microenvironment as a factor involved in phosphorylation and activation of CREB/ATF-1 in melanoma cells. In an attempt to inhibit tumor growth and metastasis of human melanoma in vivo, we have developed and generated two fully human antibodies targeting MCAM/MUC18 and IL-8. Their usage in preclinical studies will be discussed.

\section{PL 8-6}

\section{Evaluation of prognostic significance of TP53 expression signature in breast cancer}

Chikashi Ishioka, T. Ishida, T. Moriya, H. Shibata, H. Sasano, N. Ohuchi, S. Takahashi

\section{Tohoku University, Sendai, Japan}

Among previous reports, majority of them (18 of 21) has shown that TP53 mutation is worse prognostic factor in breast cancer (BC). Recent multi-center international study has shown that the TP53 mutation is a statistically independent prognostic factor among other risk factors in $\mathrm{BC}(n=1,794)$, although the predictive value for each patient is relatively low. This is probably because the frequency of the detected
TP53 mutations was low (only 17\%) and because pathogenicity of each TP53 mutation was not evaluated. We consider that more accurate method to determine TP53 status should improve the predictive value of clinical outcome of BC. Using a comprehensive expression analysis by oligo microarray and DNA sequencing of the TP53 gene in 38 Japanese BCs, we determined a gene set consisted of 33 genes from differentially expressed genes depending on the p53 status in the 26 BC (training set) and validated the ability to predict p53 status in the remaining 12 BCs (test set). Prognostic value of the gene set was analyzed in 29 BCs (stage I or II), and were validated using publicly available dataset of the independent 295 BC (stage I or II, van de Vijver et al. NEJM 2002). The gene expression signature using the 33 genes, many of which related in cell cycle and cell division, predicted the p53 status (wt or $\mathrm{mt}$ p53 signature) accurately in the test set (accuracy: $83 \%$, overall accuracy: $95 \%$ ). The p53 signature has the ability to predict relapse-free survival (RFS) of the 29 early BC (Log Rank, $P=0.032$ ). It divided the 295 early BC into $176 \mathrm{wt}$ and $119 \mathrm{mt}$ group, and also has the ability to predict both RFS and overall survival (Log Rank, both $P<0.0001)$. It also retained the predicted value in $\mathrm{ER}+, \mathrm{LN}+, \mathrm{LN}-$, St. Gallen (high) and $\mathrm{NIH}$ (high) subgroups (Log Rank, $P<0.0001)$. In conclusion, the p53 signature is a powerful and independent predictor of the outcome of disease in early breast cancer than standard systems based on clinical and histologic criteria. The data also indicates that the metastatic potential of breast cancer is acquired at a relatively early stage of the condition (initiation model, rather than progression model) and that $\mathrm{p} 53$ seems to be involved in the early stages of carcinogenesis of the mammary gland.

\section{PL 8-7}

\section{Molecular prediction for metastasis of gastrointestinal cancers with whole human gene expression analysis}

Ichiro Takemasa ${ }^{1}$, Shinichi Yoshioka ${ }^{1}$, Masakazu Miyake $^{1}$, Jun Takeno ${ }^{1}$, Nobuyoshi Kittaka ${ }^{1}$, Masataka Ikeda $^{1}$, Hirofumi Yamamoto ${ }^{1}$, Mistugu Sekimoto ${ }^{1}$, Kenichi Matsubara ${ }^{2}$, Morito Monden ${ }^{1}$

${ }^{1}$ Department of Surgery, Graduate School of Medicine, Osaka University, Osaka, Japan, ${ }^{2}$ DNA Chip Research Institute

Background The progressive accumulation of molecular changes parallels the clinical and histopathologic progression in cancer cell differentiation. Although such 
aberrant gene expression is critical for clinical cancer diagnosis, however, we lack a comprehensive understanding of whole human gene alternation. Currently armed with a few hundred molecular markers in diagnostic settings, this represents an exceptional prospect for histopathology, since it is now becoming possible to gather a more complete molecular sketch of human tumors through such an expansion of assayable markers. Purpose The aim of this study is to establish the prediction system for the metastasis and/or prognosis of gastrointestinal cancers, using DNA chip technology. Materials and Methods This study contains the two major branches, one is multicenter registration system of thousands of cancer samples and their clinicopathological information as the clinical field, and the other is comprehensive molecular analyses system of them using whole human gene $30 \mathrm{~K}$ DNA chip as the basic research field. Approximately 4,000 gastrointestinal cancer samples and their detailed pathological information and their prognosis including overall and disease free survival have been collected. Whole human gene expressions of several hundred samples of each cancer were obtained with $30 \mathrm{~K}$ DNA chip. The data mining of both huge clinicopathological and molecular information was done with sophisticated statistics. Results The candidate genes that may be related to or may play an important role for each carcinogenesis such as colorectal, gastric, hepatocellular, esophageal, and pancreatic cancer were identified. And after the recognition of the characteristic gene expression patterns in each cancer correlated with metastasis, recurrence, and prognosis, the prediction accuracies could be achieved over $80 \%$ in each cancer. Conclusions We are succeeded in the establishment of prediction system for the tumor behavior using the results of such molecular assays. Gene expression profile would help us to understand the molecular mechanisms of gastrointestinal cancers. Hence force it is expected that with such molecular approaches we can obtain a new insight and benefit for clinical cancer diagnosis and therapy which were unable to be achieved with conventional clinicopathological findings.

\section{PL 8-8}

\section{Insulin-like growth factor-I secreted from prostate stromal cells mediates tumor-stromal cell interactions of the prostate cancer}

Manabu Kawada, Hiroyuki Inoue, Tohru Masuda, Daishiro Ikeda

Numazu Bio-Medical Research Institute, Microbial Chemistry Research Center, Shizuoka, Japan
Solid tumors are composed of tumor cells and surrounding stroma including extracellular matrix, fibroblasts, macrophages, and endothelial cells. The growth of tumor cells is regulated by the stromal cells through diffusible factors and their adhesion. These tumor-stromal cell interactions significantly contribute to the growth and metastasis of some cancers such as the breast and prostate cancers. The prostate cancer shows high expression of type I insulin-like growth factor receptor (IGF-IR) and the prostate stromal cells produce insulin-like growth factor-I (IGF-I). Although high plasma level of IGF-I is a risk factor of the prostate cancer, the significance of the prostate stromal IGF-I in the regulation of the prostate cancer remains elusive. Here we show that the stromal IGF-I certainly regulates the prostate cancer development. Coinoculation of prostate stromal cells (PrSC) increased the growth of human prostate cancer LNCaP and DU-145 tumors in SCID mice. The conditioned medium of PrSC, as well as IGF-I, induced phosphorylation of IGF-IR and increased the growth of LNCaP and DU-145 cells. PrSC, but not LNCaP and DU-145 cells, secreted the significant amounts of IGFI. Coculture with PrSC increased the growth of DU145 cells in vitro, but the pretreatment of PrSC with siRNA of IGF-I did not enhance it. Furthermore, various chemical inhibitors consisting of 79 compounds with about 60 different targets led to the finding that only IGF-IR inhibitor suppressed the PrSC-induced growth enhancement of DU-145 cells. Thus, these results demonstrate that the prostate stromal IGF-I mediates tumor-stromal cell interactions of the prostate cancer to accelerate the tumor growth, supporting the idea that the IGF-I signaling is a valuable target for the treatment of the prostate cancer.

\section{PL 8-9}

\section{Expression and distribution of Aurora-B in human oral squamous cell carcinoma cell line highly metastatic to lymph node}

Akifumi Kanda ${ }^{1}$, Shiho Suto ${ }^{1}$, Sunao Sato ${ }^{2}$, Takashi Takata $^{2}$, Takahide $\mathrm{Ota}^{3}$, Masaaki Tatsuka ${ }^{1}$

${ }^{1}$ Department of Molecular Radiobiology, Hiroshima University, Hiroshima, Japan, ${ }^{2}$ Department of Oral Maxillofacial Pathobiology, Hiroshima University, Hiroshima, Japan, ${ }^{3}$ Department of Molecular Oncology and Virology, Kanazawa Medical University, Japan

Aurora-B is a key component of the chromosome passenger complex and is responsible for maintaining 
chromosomal integrity during mitosis and cytokinesis. Overexpression of Aurora-B is commonly found in human cancer. Aurora-B overexpressing stable transfectant clones derived from p53 tumor suppressordefective diploid rodent cells are tumorigenic and metastatic. Here we report that cytoplasmic expression of Aurora-B is closely related to lymph node metastatic potential in human oral squamous cell carcinoma lines. Our established HSC-3-3M cells, highly metastatic to cervical lymph node, up-regulated Aurora-B expression, particularly in the soluble cytoplasmic fraction compared with their parental HSC-3 cells which had lower metastatic potential. Using confocal laser microscopy, in HSC-3-3M cells expressing enhanced green fluorescence protein fused Aurora-B, we found that Aurora-B distributes in the lamellipodia and the cortex, in addition to nuclear localization, in interphase cells. These results implicate overexpression of cytoplasmic Aurora$\mathrm{B}$ in augmenting metastatic potential, and represent further evidence for the important role of Aurora-B in cancer progression via signal transduction perturbations operating in conjunction with chromosome segregation errors.

\section{PD 1-1}

\section{A vascular maturation signal that suppresses endothelial MT1-MMP expression}

\section{Ikuo Yana, Motoharu Seiki}

Division of Cancer Cell Research, Institute of Medical Science, University of Tokyo, Tokyo, Japan

The membrane-anchored matrix metalloproteinase (MMP), MT1-MMP, plays a key role in the angiogenic process, but the mechanisms underlying its spatiotemporal regulation in in vivo have not been fully defined. Using whole mount immunohistochemical analysis and a beta-galactosidase transgene inserted into the murine MT1-MMP gene, we have previously reported that MT1-MMP expression in vivo is largely concentrated to the sprouting tip of both in vitro and in vivo neocapillary. To elucidate the regulation mechanism, we employed set of co-culture models with a human aortic smooth muscle cell and umbilical endothelial cell. 3D immunohistochemical analysis for MT1-MMP localization in the capillary network expressed a mere MT1MMP, while only tip cells, which was locally untheathed with VSMC, expressed MT1-MMP. Interestingly, MT1MMP induction in the 3D network formation was wholly enhanced, regardless of the tip and stalk, by the treatment of soluble TIE2 which expectedly blocked the Ang-1 mediated signal. Consistently, in ex vivo assay or in vivo angiogenesis assay with the gene targeted mice, the lacZ activity was enhanced whole through the neocapillary only when the soluble TIE2 was administrated. Thus it was suggested that the intrinsic and site-specific MT1-MMP expression was controlled by endothelial cell-smooth muscle cell (SMC) crosstalk. When vascular maturation induced by SMC-derived angiopoietin-1 was inhibited with recombinant soluble TIE-2, MT1MMP distribution was no longer restricted to the endothelial tip cells, but instead redistributed throughout the neovessel network. Taken together, these data demonstrate that vascular maturation coordinated by endothelial cell/mural cell interactions redirects MT1MMP expression to the neovessel tip where the protease regulates matrix remodeling at the leading edge of the developing vasculature.

\section{PD 1-2}

\section{Calcium influx-phospholipase D-MAP kinases (ERK and $\mathrm{p38}$ )-NF $\kappa \mathrm{B}$ pathway and acidic sphingomyelinase pathway mediate the acidic extracellular $\mathrm{pH}$ signaling to induce MMP-9expression}

Yasumasa Kato $^{1,2}$, Shigeyuki Ozawa ${ }^{3}$, MamoruTsukuda ${ }^{2}$, Kaoru Miyazaki ${ }^{4}$, Yves St-Pierre ${ }^{5}$, Ryu-IchiroHata ${ }^{1}$

${ }^{1}$ Department of Biochemistry and Molecular Biology, Kanagawa Dental College, Yokosuka, Japan, ${ }^{2}$ Department of Biology and Function in the Head and Neck, Yokohama City University School of Medicine, Yokohama, Japan, ${ }^{3}$ Department of Oral and Maxillofacial Surgery, Kanagawa Dental College, Yokosuka, Japan, ${ }^{4}$ Division of Cell Biology, Kihara Institute for Biological Research, Yokohama City University, Yokohama, Japan, ${ }^{5}$ INRS-Institut Armand-Frappier, University of Quebec, Quebec, Canada

Extracellular $\mathrm{pH}(\mathrm{pHe})$ is known to be acidic and modulate some gene expressions in tumor cells. We have found that acidic $\mathrm{pHe}$ induces matrix metalloparoteinase-9 (MMP-9) expression through phospholipase D (PLD)-MAP kinases (ERK and $\mathrm{p} 38$ )-NF $\kappa \mathrm{B}$ signaling (Kato et al ., J. Biol. Chem. 280, 10938-10944, 2005) . In the present study, we report that $\mathrm{Ca}^{2+}$-PLD and acidic sphingomyelinase (SMase) pathways contribute to induce MMP-9 expression at acidic pHe. BAPTA-AM, a chelator of intracellular free calcium $\left(\left[\mathrm{Ca}^{2+}\right] i\right)$ dosedependently reduced acidic pHe-induced MMP-9 expression. To determine contribution of $\left[\mathrm{Ca}^{2+}\right] i$ to this signaling, we used Fluo-4-AM, a probe of $\left[\mathrm{Ca}^{2+}\right] i$. AcidicpHe transiently increased $\left[\mathrm{Ca}^{2+}\right] i$ level in the presence but not in the absence of extracellular free 
calcium or EGTA treatment. T-type voltage-dependent calcium channel (VDCC) blocker (mibefladil) inhibited more efficiently MMP-9 expression than L-type VDCC blockers (nimodipine, SR33557) did. Their IC50 doses for suppression of acidic $\mathrm{pHe}$-induced MMP9 expression was about $1 \mu \mathrm{M}$ for mibefladil, $3 \mu \mathrm{M}$ for nimodipine, and $12.5 \mu \mathrm{M}$ for SR33557. When effect of their channel blockers on $\left[\mathrm{Ca}^{2+}\right] i$ was tested, $2.5 \mu \mathrm{M}$ mibefladil and $5 \mu \mathrm{M}$ nimodipine reduced calcium influx but $25 \mu$ M SR33557 did not. Furthermore, U73122, a broad type inhibitor for phospholipase $\mathrm{C}$, hardly affected transient increase in $\left[\mathrm{Ca}^{2+}\right] i$. These results suggested that acidic $\mathrm{pHe}$ induces calcium influx through VDCC (mainly T-type) to induce MMP-9 expression. On the other hand, SR33557 which reduced acidic $\mathrm{pH} e$-induced MMP-9 expression but did not affect calcium influx is also known to inhibit activity of acidic SMase, we used another type of inhibitors (despramine and perhexiline) for this enzyme. Despramine and perhexiline reduced acidic $\mathrm{pH} e$-induced MMP-9 expression with an IC50 values of 0.5 and $6.0 \mu \mathrm{M}$, respectively. Acidic SMase activity was induced by acidic $\mathrm{pHe}$ and this activation was attenuated by these inhibitors. To mimic acidic SMase activation by acidic $\mathrm{pH} e$, cells were treated with cell permeable C2-ceramide, an analog of acidic SMase metabolite at neutral $\mathrm{pH} e$, showing that MMP-9 expression was induced by $\mathrm{C} 2$-ceramide only in the presence of thapsigargin, a releaser of $\left[\mathrm{Ca}^{2+}\right] i$. BAPTAAM reduced acidic $\mathrm{pHe}$ induced PLD activity but not acidic SMase activity. Acidic SMase inhibitor partially reduced acidic $\mathrm{pH} e$-induced $\mathrm{NF} \kappa \mathrm{B}$ activity but not affect MAP kinase activation which were activated in the downstream PLD. These data suggested that both $\left[\mathrm{Ca}^{2+}\right] i$-triggered PLD-MAP kinase pathway and acidic SMase pathway regulate $\mathrm{NF} \kappa \mathrm{B}$ activation to induce MMP-9 expression.

\section{PD 1-3}

\section{Regulation of MMP-2 activation by syndecan-2 through its heparan sulfate side chains in Lewis lung carcinoma cells}

Seiichi Munesue ${ }^{1}$, Yasuo Yoshitomi ${ }^{1}$, Yoshie Koyama ${ }^{1}$, Shinsuke Kiyono ${ }^{1}$, KayokoOguri ${ }^{2}$, Hayao Nakanishi ${ }^{3}$, Satoru Shimizu ${ }^{1}$, MinoruOkayama ${ }^{1}$

${ }^{1}$ Department of Biotechnology, Faculty of Engineering, Kyoto Sangyo University, Kyoto, Japan, ${ }^{2}$ Clinical Research Center, National Hospital Organization Nagoya Medical Center, Nagoya, Japan, ${ }^{3}$ Division of Oncological Pathology, Aichi Cancer Center Research Institute, Nagoya, Japan
Metastatic process consists of a number of distinct steps. One of the critical steps is degradation of the basement membranes underlying the epithelial and endothelial cell layers by tumor cells. Using Lewis lung carcinoma-derived different metastatic clones (P29, LM12-3 and H11 with low, intermediate and high metastatic potentials, respectively), we have previously demonstrated that there is an inverse correlation between expression of a cell surface heparan sulfate proteoglycan, syndecan-2, and metastatic potential. The causal relationship behind this correlation was demonstrated by transfection of syndecan-2 core protein cDNA into H11 cells. Overexpression of syndecan-2 caused great suppression of metastasis. In order to elucidate a mechanism underlying this relationship, we analyzed gelatinases (MMP-2 and MMP-9) known to be involved in degradation of the basement membranes in metastasis. All the clones used expressed the similar levels of MMP-2 regardless of the differences of their metastatic potentials, but did not express MMP-9 significantly. Interestingly, we found that an activation degree of MMP-2 was inversely correlated with the syndecan-2 expression. Overexpression of syndecan-2 in H11 cells caused the suppression of its activation correspondingly to its expression level. Furthermore, we found that the expression levels of the molecules involved in the activation of MMP-2, such as TIMP-2 and MT 1-MMP, were very similar among all the clones used. To examine a direct involvement of syndecan-2 in suppression of MMP-2 activation, we examined a binding capacity of MMP-2 to heparin. The result obtained showed that MMP-2 exhibited an avidity to heparin at $\mathrm{Kd}=6.2 \times 10^{-8} \mathrm{M}$. We then tested a possibility of participation of heparan sulfate chains of syndecan-2 in suppression of MMP-2 activation. In P29 cells treated with heparitinase, MMP-2 activation was significantly enhanced. On the other hand, MMP-2 activation was suppressed by overexpression of the wild type syndecan2, whereas transfection of a mutated syndecan-2 cDNA lacking glycosaminoglycan attachment sites into $\mathrm{H} 11$ cells, named as H11-SN2 $\delta$ GAG cells, did not cause such suppression, indicating that the suppression was caused by heparan sulfate side chains of syndecan-2. Consistent with this finding, the metastatic potential of H11-SN2 $\delta$ GAG cells was greatly reduced as compared with that of the parent $\mathrm{H} 11$ cells. These results clearly show that in this model, syndecan-2 heparan sulfate chains suppress the activation of MMP2 and cause the suppression of metastasis. This conclusion is consistent with the accumulated results that heparin efficiently suppresses metastasis of various tumor cells. 


\section{PD 1-4}

MMP-2 activated by MT1-MMP plays an important role in tumor invasion

Tomoya Kudo, Hisashi Miyamori, Takahisa Takino, Hiroshi Sato

Department of Molecular Virology and Oncology, Cancer Research Institute, Kanazawa University

Matrix metalloproteinase (MMP)-2 is known to be activated through the formation of tri-molecular complex consist of membrane-type MMP-1 (MT1-MMP), tissue inhibitor of MMP (TIMP)-2 and MMP-2. However, whether MMP-2 activated in the presence of TIMP-2 has enzymatic activity or not has not been examined. In this study, HEK293T cells which produce a trace amount of TIMP-2 were co-transfected with MT1-MMP expression plasmid and variable amounts of TIMP-2 plasmid, and cells were incubated with MMP-2 for $1 \mathrm{~h}$ at $48 \mathrm{~h}$ after transfection. MMP-2 incubated with the cells transfected with MT1-MMP plasmid alone was processed only slightly to the active form, and showed a low level gelatindegrading activity. In contrast, MMP-2 was converted from latent to active form by the cells co-transfected with TIMP-2 plasmid of both low and high concentration, however, gelatin-degrading activity was demonstrated only with MMP-2 activated by the cells co-trasnfected with lower concentrations of TIMP-2 plasmid. MMP-2 activated by HT 1080 cells showed high gelatin-degrading activity. HT1080 cells co-cultured with HEK293T cells expressing MMP-2 in 3-dimensional collagen gel migrated to the out of the gel more effectively than HT080 cells co-cultured with control HEK 293T cells. These results suggest that MMP-2 activated by MT1-MMP in the presence of an appropriate concentration of TIMP-2 contributes to tumor invasion.

\section{PD 1-5}

Increased host MMP 9 expression in tumors and ovaries of mice bearing ovarian carcinoma cells overexpressing VEGF

Dorina Belotti, Catia Calcagno, Angela Garofalo, Luigi Manenti, Massimo Broggini, Giulia Taraboletti, Raffaella Giavazzi

Department of Oncology, Mario Negri Institute for Pharmacological Research, Bergamo, Italy

Tumor development is the result of a complex functional crosstalk between transformed and stromal cells.
In particular Vascular Endothelial Growth Factor (VEGF) and Matrix Metalloproteinases (MMPs) mutually regulate each other, contributing to tumor progression. We have previously reported that MMPs regulate tumor VEGF bioavailability and ascites formation in ovarian carcinoma [1]; conversely, VEGF is known to stimulate MMP production.

To further investigate the functional interplay between VEGF and MMPs in ovarian carcinoma progression, we have established a model of human ovarian carcinoma cells that overexpress VEGF and disseminate in the peritoneal cavity of nude mice. Xenograft variants derived from the A 2780 human ovarian carcinoma (1A9), stably expressing VEGF121 in sense (1A9-VS-1) and antisense orientation (1A9VAS-3) were generated and the invasive and metastatic potential of tumor cells producing different levels of VEGF evaluated. 1A9-VS1 and 1A9-VAS-3 disseminated in the peritoneal cavity of nude mice, but only 1A9-VS-1, the VEGF121 overexpressing tumor variant, produced ascites and specifically invaded ovaries. Significant levels of soluble VEGF were detected in plasma of mice bearing 1A9-VS1 tumors.

Augmentation of murine MMP9 expression was observed in tumors and ovaries of mice transplanted with 1A9-VS-1 compared to mice bearing 1A9-VAS-3 tumors, indicating that VEGF produced by tumor cells stimulates host expression of the matrix-degrading enzyme. Notably, modulation of host MMP9 activity was organ specific and the levels of host proMMP-9 in ovaries correlated with the plasma levels of VEGF produced by tumor cells.

These findings point to a complex cross-talk between tumor VEGF and stromal MMPs in determining ovarian tumor progression.

Supported by the European Union FP6, LSHC-CT2003503297.

\section{Reference}

1. Belotti et al (2003) Cancer Res 63:5224-5229

\section{PD 1-6}

Processing of the human laminin $\gamma 2$ chain by membrane-type-1 matrix metalloproteinases (MT 1-MMP) and its proteolytic fragment potentially supports cancer malignant progression

Naohiko Koshikawa ${ }^{1}$, Shanshan Liu ${ }^{2}$, Vito Quaranta ${ }^{2}$, Motoharu Seiki ${ }^{1}$ 
${ }^{1}$ Division of Cancer Cell Res., Inst of Med. Sci., Univ. of Tokyo, Tokyo, Japan, ${ }^{2}$ Department of Cancer Biology, Vanderbilt University School of Medicine

Activation of EGF receptor (EGF-R) signaling is an important step in tumor progression. However, availability of EGF-R ligands in the tumor microenvironment is not well understood. We have previously demonstrated that processing of laminin $\gamma 2$ by membrane-type- 1 matrix metalloproteinase (MT1-MMP) released an EGF-like domain of $\gamma 2$ chain called domain III (DIII) fragment and its fragment is a potential of EGF-R ligand. Here we show that DIII fragment promotes resistance to adherence dependent apoptosis in OVCAR-8, a human ovarian cancer cell line. Key to this process is the expression by OVCAR- 8 of a monomeric $\gamma 2$ chain. Knock-down of $\gamma 2$ by siRNA abolishes resistance to apoptosis in vitro and significantly decreases growth of OVCAR-8 tumor xenografts. Addition of purified monomeric $\gamma 2$ to tumor cells caused resistance to apoptosis that was abolished by MMP or EGF-R inhibitors, consistent with the fact that DIII fragment is proteolytically generated from $\gamma 2$ by MT1-MMP and that it activates EGF-R. These results provide a possible explanation for the reported association between worsened prognosis and monomeric $\gamma 2$ chain localization at the leading edge of invasive tumors of several histological types. Taken together, these suggest that MT1MMP processing of monomeric $\gamma 2$ chain in human tumors may stimulate the EGF-R resulting in increased tumorigenicity and protection from apoptosis that could possibly increase the malignant potential of tumors.

\section{PD 1-7}

\section{MT1-MMP drives cancer cell invasion and intravasation in vivo}

Ichiro Ota ${ }^{1,2,3,4}$, Farideh Sabeh $^{2}$, Nariaki Matsuura ${ }^{3}$, Katsunari Yane ${ }^{4}$, Hiroshi Hosoi ${ }^{4}$, Stephen Weiss ${ }^{2}$

${ }^{1}$ Department of Otolaryngology, Tenri Municipal Hospital, Nara, Japan, ${ }^{2}$ Division of Molecular Medicine and Genetics, Department of Internal Medicine, University of Michigan, Ann Arbor, MI, USA, ${ }^{3}$ Molecular Pathology, Division of Allied Health Sciences, Osaka University Graduate School of Medicine, Osaka, Japan, ${ }^{4}$ Department of Otolaryngology, Nara Medical University, Nara, Japan

As cancer cells traverse collagen-rich extracellular matrix (ECM) barriers and intravasate, they adopt a fibroblast-like phenotype and engage undefined proteolytic cascades that mediate invasive activity. Herein, we report that fibroblasts and tumor cells are able to tunnel through dense barriers of cross-linked type I collagen in vivo as well as in vitro via a virtually indistinguishable proteolytic process that requires matrix metalloproteinase (MMP) activity. Further, we demonstrate that fibroblasts deficient for the membrane-anchored metalloproteinase MT1-MMP are unable to display pericellular collagenolytic activity and are incapable of expressing a collagen-invasive phenotype in vivo, using chick chorioallantoic membrane (CAM) assay. Likewise, gene silencing of MT1-MMP in human carcinoma or fibrosarcoma cells similarly results in a complete loss of membrane-associated collagenolytic activity and renders tumor cells unable to infiltrate host tissues. These data identify MT1-MMP as a required pericellular collagenolysin that confers both normal and neoplastic cells with tissue-invasive activity in vivo.

\section{PD 1-8}

\section{Membrane-type 1 matrix metalloproteinase modulates focal adhesion stability and cell migration}

Takahisa Takino $^{1}$, Hisashi Miyamori ${ }^{1}$, Hiroshi Sato ${ }^{1}$

${ }^{1}$ Department of Molecular Virology and Oncology, Cancer Research Institute, Kanazawa University, Kanazawa, Japan

Membrane-type 1 matrix metalloproteinase (MT1-MMP) plays an important role in extracellular matrix-induced cell migration and the activation of extracellular signalregulated kinase (ERK). We showed here that transfection of the MT1MMP gene into HeLa cells promoted fibronectin-induced cell migration, which was accompanied by fibronectin degradation and reduction of stable focal adhesions, which function as anchors for actin-stress fibers. MT1-MMP expression attenuated integrin clustering that was induced by adhesion of cells to fibronectin. The attenuation of integrin clustering was abrogated by MT1-MMP inhibition with a synthetic MMP inhibitor, BB94. When cultured on fibronectin, HT1080 cells, which endogenously express MT1-MMP, showed so-called motile morphology with well-organized focal adhesion formation, well-oriented actin-stress fiber formation and the lysis of fibronectin through trails of cell migration. Inhibition of endogenous MT1-MMP by BB94 treatment or expression of the MT1-MMP carboxyl-terminal domain, which negatively regulates MT1-MMP activity, resulted in the suppression of fibronectin lysis and cell migration. BB94 treatment promoted stable focal adhesion formation concomitant with enhanced phosphorylation of tyrosine 
397 of focal adhesion kinase (FAK) and reduced ERK activation. These results suggest that lysis of the extracellular matrix by MT1-MMP promotes focal adhesion turnover and subsequent ERK activation, which in turn stimulates cell migration.

\section{PD 1-9}

\section{Participation of glypican-like proteoglycans in localization of MMP-9 on the cell surface of colon carcinoma cells}

Yasuo Yoshitomi ${ }^{1}$, Hideaki Naruo ${ }^{1}$, Seiichi Munesue ${ }^{1}$, Yoshie Koyama $^{1}$, Shinsuke Kiyono ${ }^{1}$, Katsunori Hashimoto $^{2}$, Toyoharu Yokoi ${ }^{2}$, Satoru Shimizu ${ }^{1}$, KayokoOguri $^{3}$, Minoru Okayama ${ }^{1}$

${ }^{1}$ Department of Biotechnology, Faculty of Engineering, Kyoto Sangyo University, Kyoto, Japan, ${ }^{2}$ Department of medical Technology, Nagoya University School of Health Sciences, Nagoya, Japan, ${ }^{3}$ Clinical Research Center, Nagoya Hospital Organization Nagoya Medical Center, Nagoya, Japan

Matrix metalloproteinases (MMPs), which could degrade a variety of matrix proteins, have been reported to be involved in many pathophysiological processes including tumor cell invasion and metastasis. Secretedtype MMPs are released into extracellular space as a latent proMMP molecular form, and could play important roles in various physiological phenomena through complex regulations of their expression, activation and inhibition. However, as to MMP-9, the compartment and mechanism of its activation have not been fully understood. As the first step to elucidate the mechanism, in the present study, cellular localization of MMP-9 and its roles in cell migration and invasion were examined using LuM1 and NM11 clones with high and low metastatic potentials, respectively, which were established from murine colon adenocarcinoma cells on the basis of their spontaneous metastatic potentials. LuM1 clone had higher ability of cell migration and invasion through Matrigel as compared to NM11 one, and secreted remarkably larger amounts of MMP-9 than NM11. Flow cytometrical and immunohistochemical analyses showed that MMP-9 was associated with the cell surface of LuM1 cells, and condensed on the lamellipodia of the plasma membranes. Both the latent and active forms of MMP-9 had high affinity to the heparin-Sepharose, an affinity of the active form being higher than that of proform. Treatment of the cells with heparitinase I or exogenous addition of heparin to the cells resulted in release of the MMP-9 from the cell surface. Reflecting these phenomena, cell migration and invasion were inhibited by these treatments. The MMP-9 inhibitor also inhibited these cell behaviors. Analysis of the core protein of heparan sulfate proteoglycans revealed that LuM1 cells contained a variety of heparan sulfate proteoglycans including syndecans, CD44 and glypicans. Of these heparin sulfate proteoglycans, the proteoglycans with their molecular core protein size of approximately 66 and $64 \mathrm{kDa}$ were co-precipitated with MMP-9 by anti-MMP-9 antibody. These MMP-9 anchoring proteoglycans were released from the cells by digestion with phosphatidylinositolspecific phospholipase $\mathrm{C}$. These results indicate that secreted MMP-9 associates with the glypican-like proteoglycan through its heparan sulfate chains, and plays a important role in the cell migration, invasion, and metastasis.

\section{PD 1-10}

\section{A novel enhanced lung colonizing variant of the murine bladder cancer cells}

Minoru Horinaga $^{1,3}$, Ryuichi Fukuyama ${ }^{2}$, Kelley Harsch $^{2}$, Muzaffer Cicek ${ }^{2}$, Warren Heston ${ }^{2}$, William Larchian $^{3}$

${ }^{1}$ Department of Urology, Fussa hospital, Tokyo, Japan, ${ }^{2}$ Department of Cancer Biology, The Cleveland Clinic Foundation, Cleveland, Ohio, USA, ${ }^{3}$ Department of Glickman Urological Institute, The Cleveland Clinic Foundation, Cleveland, Ohio, USA

Objective Despite efforts to develop a metastatic model of bladder cancer in which tumor invasion and metastasis can be investigated, little progress has been made. This study was designed to develop an enhanced lung colonizing variant of murine bladder cancer that will allow the mechanism of metastasis to be studied more readily. Methods We implanted murine bladder tumor cells (MBT-2) into the leg muscles of $\mathrm{C} 3 \mathrm{H}$ mice. We developed variant cells from lung metastasis nodule. We compared the MBT-2 cells and variant cells (MBT$2 \mathrm{~V}$ ) in vivo by evaluating lung nodule formation, survival, and in vitro adhesion, and migration-invasion assays. Zymograpy and semi-quantitative reverse transcriptase-polymerase chain reaction (RT-PCR) analyses were also performed to characterize the metastatic ability of both cells. Results When a tumor diameter was observed greater than $20 \mathrm{~mm}$, the mouse was sacrificed, and the metastatic lesions were aseptically removed from the lungs, minced, and then cultured in vitro . This cycle was repeated twice. These isolated cells were named the enhanced lung colonizing variant cells, MBT-2V. MBT-2 cells and MBT-2V were 
tumorigenic when injected intramuscularly into $\mathrm{C} 3 \mathrm{H}$ mice, but MBT-2 cells had little potential to metastasize than did the MBT-2V. Metastases were observed in the lungs of mice injected in the tail vein with MBT-2 cells and MBT-2V. Mice receiving MBT-2V had significant shorter survival $(P<0.01)$ and more lung nodules at day 12 following tail vein injection (245 vs. 106, $P<0.0001)$ than those receiving MBT-2 cells. In vitro study revealed that MBT-2V exhibited more adhesion, higher migration and invasiveness than did MBT-2 cells. Pathological examination indicated the tumors to be more aggressive in MBT-2V than in MBT-2 cells. MBT$2 \mathrm{~V}$ also showed significantly higher matrix metalloproteinase (MMP)-9 expression.

Conclusions: We generated enhanced lung colonizing variant cells of MBT-2 cells. Our MBT-2V showed more aggressive and invasive metastatic ability than that of MBT-2 cells. Zymography and RT-PCR analyses indicated that MMP-9 might be associated with metastatic ability of MBT-2V.

\section{PD 1-11}

\section{Hyaluronic acid induces nuclear factor $\kappa \mathbf{B}$-mediated metalloproteinase-9 Secretion through $\mathbf{I} \kappa \mathbf{B} \alpha / \mathrm{IKK}$ signaling pathways, and 17-AAG down-regulates these pathways}

Mi Suk Kim, JiWoo Lee, Heon Yoo, Sang Hoon Shin, Seung Hoon Lee

Research Institute and Hospital, National Cancer Center, Goyang, Gyeonggi, Korea

We have recently reported that hyaluronic aicd (HA) stimulates secretion of matrix metalloproteinase-9 (MMP-9) through nuclear factor- $\kappa \mathrm{B}(\mathrm{NF}-\kappa \mathrm{B})$ in glioma cells [1]. However, the molecular mechanism by which HA activates NF- $\kappa$ Band regulates MMP-9 secretion in glioma cells is not well defined. We also investigated the mechanism of action of 17-AAG, a known Hsp90 inhibitor agent on HA-induced NF- $\kappa$ B-mediated secretion of MMP-9 in glioma cells. Here we report that HA induces phosphorylation and degradation of the inhibitor of nuclear factor- $\kappa \mathrm{B}(\mathrm{I} \kappa \mathrm{B} \alpha)$ by inducing the activity of $\mathrm{I} \kappa \mathrm{B}$ kinase (IKK) in these cells. HA also induces the nuclear accumulation of NF- $\kappa \mathrm{B}$ p 65 and NF- $\kappa$ B-DNA binding. However, 17-AAG suppressed HA-induced I $\kappa$ $\mathrm{B} \alpha$ phosphorylation and degradation by inhibiting the IKK activity. Moreover, our data revealed that 17AAG inhibited the HA-induced translocation of NF- $\kappa$ B p65 and NF- $\kappa$ B-DNA binding. The HA-induced MMP9 secretion was also drastically reduced by $17-\mathrm{AAG}$. 17AAG also inhibited HA-induced cell migration and extracellular matrix invasion. Taken together, these results demonstrate that HA induces $\mathrm{NF}-\kappa$ B activity through phosphorylation and degradation of $\mathrm{I} \kappa \mathrm{B} \alpha$ by activating IKK that ultimately triggers the secretion of MMP-9, and further demonstrates that 17-AAG potently suppresses HA-induced cell migration, invasion, and NF- $\kappa$ B-mediated MMP-9 secretion by blocking the $\mathrm{IKK} / \mathrm{I} \kappa \mathrm{B} \alpha$ signaling pathways.

\section{Reference}

1. Kim MS et al (2005) Int J Oncol 27:839-846

\section{PD 2-1}

\section{Novel sialidase NEU4 suppresses cell motility of colon cancer cells through attenuation of E-selectin-derived signaling}

Kazuhiro Shiozaki ${ }^{1}$, Hideaki Yamanami ${ }^{2}$, Takuji Uemura $^{1}$, Tadashi Wada ${ }^{1}$, Kazunori Yamaguchi ${ }^{1}$, Taeko Miyagi ${ }^{1}$

${ }^{1}$ Division of Biochemistry, Miyagi Cancer Center Research Institute, CREST, JST, Miyagi, Japan, ${ }^{2}$ Division of Surgery, Miyagi Cancer Center Research Institute, CREST JST, Miyagi, Japan

Sialidase catalyzes the removal of sialic acid from glycoproteins and gangliosides. Four mammalian sialidases (Neu1-4) have been so far cloned and characterized. The human sialidase NEU4 recently explored is different from other sialidases in substrate specificity as well as in subcellular localization. To elucidate the pathophysiological function of NEU4, we investigated alteration of NEU4 expression and its role in colon cancer, since NEU4 was found to be relatively rich in human colon mucosae. In comparison with adjacent non-cancerous mucosae, colon cancer tissues showed marked decrease in the mRNA level, in contrast to the case of NEU3. To understand the significance of the decrease, we observed if malignant cell properties could be reversed by NEU4 gene introduction into colorectal cancer cell lines. Colony formation on soft agar, cell motility and invasion were significantly reduced in NEU4 transfected cells. When sialic acid contents in the transfectants were assessed with HPLC, NEU4 removed both lipid- and protein-bound sialic acids, but the latter was remarkably decreased. Flow cytometry analyses showed that the levels of sialyl-Le ${ }^{\mathrm{a}}$ and sialyl-Le ${ }^{\mathrm{x}}$ were declined by NEU4 transfection. Cell attachment and growth on E-selectin was significantly inhibited in NEU4 transfected cells. We then examined whether the decrease 
of sialyl-Le ${ }^{\mathrm{a}}$ and sialyl-Le ${ }^{\mathrm{x}}$ affected especially cell motility. Supplementation of E-selectin in motility assay remarkable cell migration, but the increase was abrogated after NEU4 transfections. Considering the possibility that suppression of cell motility by NEU4 was caused by attenuation of signaling through sialyl-Le $\mathrm{e}^{\mathrm{a}}$ and sialyl- $\mathrm{Le}^{\mathrm{x}}$, phosphorylation levels of related molecules were evaluated. p38 and ERK phosphorylation level was increased after Eselectin stimulation, whereas this effect was hardly detected in NEU4 transfected cells. Although SAPK phosphorylation level was not changed by NEU4 transfection, phosphorylation of Hsp27, downstream of p38, and actin re-organization were also suppressed, suggesting that inhibition of actin reorganization might be involved in reduction of cell motility by NEU4. These results indicate that NEU4 is involved in suppression of the malignant phenotype of the colon cancer cells, which is opposite to NEU3 effects.

\section{PD 2-2}

\section{Functional analysis of junctional adhesion molecule-C (JAM-C) in cancer metastasis}

Yuuki Ishida, Chiaki Fuse, Tomoya Hikita, Kosuke Shimizu, Tomohiro Asai, Naoto Oku

Department of Medical Biochemistry and COE 21, School of Pharmaceutical Sciences, University of Shizuoka, Japan

Junctional adhesion molecules (JAMs) are localized in the tight junction of polarized epithelial cells. In addition, JAMs are also expressed on the surface of endothelial cells, circulating leukocytes, and platelets. Four types of JAMs (JAM-A, -B, -C, -D) have been identified at present. Most of JAMs are known to be implicated in inflammation, in particular, during a process called transendothelial migration, or diapedesis. In the present study, we examined implications of JAMs in cancer metastasis.

Previously, we presented that JAM-C was expressed in most of tumors having potent metastatic properties and human JAM-C (hJAM-C) overexpression in HT1080 cells promotes adhesion of cancer cells to ECM components, invasion and motility in vitro experiment. In the present study, we examined the role of JAM-C in an in vivo experimental metastatic model using HT1080 cells. The results showed the overexpression of hJAM-C in HT1080 cells significantly decreased life spans of $\mathrm{BALB} / \mathrm{c} \mathrm{nu} / \mathrm{nu}$ mice, suggesting that hJAM-C might be correlated with tumor malignancy. In addition, since HT1080 cells have a little endogenous hJAM-C, we performed the knockdown of endogenous JAM-C by RNA interference (RNAi) strategy to confirm whether endog- enous hJAM-C was involved in the invasion processes. Transfection of HT1080 cells with JAM-C small interfering RNA reduced their adhesiveness to ECM components, invasiveness and motility in vitro. The results suggested that endogenous JAM-C would promote tumor invasiveness, although the precise mechanism has not been elucidated. Since the cytoplasmic region of JAM-C has PKC phosphorylation sites and PDZ binding motif involved in signal transduction, we hypothesized that signal transduction in JAM-C is involved in this mechanism. To investigate the roles of cytoplasmic region of JAM-C in cancer metastasis, we established three types of hJAM-C mutants that have truncated cytoplasmic domain and expressed them on HT 1080 cells. Overexpressions of hJAM-C mutants in HT 1080 cells were confirmed by Western blotting. By use of these transfectants, we performed invasion assay. The results showed that the invasion of HT 1080 cells was significantly accelerated by the overexpression of cytoplasmic domain deletion mutants of hJAM-C.

These data indicate that the expression of JAM-C promotes cancer metastasis by enhancing the adhesion of cancer cells to ECM components and subsequent invasion and the importance of JAM-C extracellular domain for the promotion of cancer cell invasion. Our present study is the first report demonstrated that JAM-C expression promotes tumor malignancy and suggests that JAM-C may be a target molecule for blocking cancer metastasis.

\section{PD 2-3}

\section{Evaluation of cell-cell adhesion related proteins as prognostic markers for cervical cancer metastasis}

Jelmer J. van Zanden ${ }^{1}$, Esther R. Nijhuis ${ }^{1}$, Haukeline H. Volders ${ }^{1}$, Klaske A. Ten Hoor ${ }^{1}$, G. Bea A. Wisman ${ }^{1}$, Harry Hollema $^{2}$, Ed Schuuring ${ }^{2}$, Ate G. J. van der Zee ${ }^{1}$

${ }^{1}$ Department of Gynecological Oncology, University Medical Center, University of Groningen, The Netherlands, ${ }^{2}$ Department of Pathology, University Medical Center, University of Groningen, The Netherlands

Presence of pelvic lymph node metastasis is the most important negative prognostic factor for women with early stage cervical cancer. Using an expression profiling approach we identified several genes involved in cell adhesion and $\beta$-catenin signaling which were differentially expressed between cervical tumors of patients with positive lymph nodes (e.g. metastases) versus patients with negative lymph nodes. To explore the prognostic value of these proteins we studied the prognostic value of a selection of these proteins using immunohisto- 
chemical analysis on a tissue microarray (TMA) containing 146 stage IB/IIA cervical carcinomas. For all patients presented on the TMA complete clinicopathologic and follow-up data are available to allow prognostic analyses. The proteins studied were E-cadherin, $\beta$-catenin and p120ctn (important cell adhesion proteins), together with MMP7 and cyclin D1 as downstream targets of $\beta$ catenin signaling. Besides overall staining intensity of the different targets also the cellular localization was examined since this has been reported extensively as an important factor affecting cellular behavior. Loss or down regulation of E-cadherin releases proteins, such as $\beta$-catenin and p120ctn, which are known to regulate transcriptional activity, from a membrane-bound state into the cytoplasm. Preliminary results indicate that especially E-cadherin and p120ctn are associated with metastasis, as indicated by changes in both the overall intensity as well as the cellular localization. Further analyses will reveal the prognostic value of these proteins for cervical cancer patients. Moreover, the results will provide more insight into the biological mechanisms causing lymph node metastasis in early stage cervical cancer. This research is financially supported by The Dutch Cancer Society grant RUG

\section{PD 2-4}

\section{Vascular endothelial growth factor promotes proliferation and invasion in bladder cancer cell lines}

Ryoichi Nakanishi, Yoshihiro Kusuhara, Ryuichi Taue, Kunihisa Yamaguchi, Hiroyoshi Nakatsuji, Tomoteru Kishimoto, Natsuo Oka, HirofumiIsaki, Masayuki Takahashi, Tomoharu Fukumori, Masa-aki Nishitani, Hiro-omi Kanayama

Department of Urology, The University of Tokushima Graduate School of Health, Japan

Purpose Invasive bladder cancer generally has high graded malignancy and poor prognosis. Currently we treat patients with muscle invasive bladder cancer by chemotherapy and radical cystectomy, with limited therapeutic effect. VEGFR is tyrosine kinase for VEGF, a key regulator of angiogenesis in various cancers. It is well-known that VEGF targeted therapy is effective in various malignancies. And in bladder cancers VEGF targeted therapy may induce tumor response and may prolong survival. In this study, we evaluated the relationship between VEGF and VEGF receptor in bladder cancer cell lines, and the effectiveness of VEGFR inhibitor against tumor cell lines. Materials and methods In highly invasive bladder cancer cell lines (T-24, UMUC-3 and J-82) we measured mRNA expression of
VEGF, VEGF receptor 1 and VEGF receptor 2 by using quantitative RT-PCR. The expression of VEGF receptors 1 and 2 were evaluated by Western blotting. We assessed the effect of VEGF on proliferation and invasion of bladder cancer cell lines. The effect of VEGFR inhibitor against bladder cancer cell lines was also measured. Results VEGF increased tumor proliferation in dose dependent manner in all cell lines. VEGF also increased tumor invasion. VEGF inhibitor inhibited proliferation. Conclusions This study suggested that in bladder cancer cell lines VEGF promoted tumor proliferation and invasion through VEGF receptor. VEGF targeted therapy may be effective in treating invasive bladder cancers.

\section{PD 2-5}

\section{Expression of semaphorin 5A in metastatic pancreatic cancer cells and modulates cellular proliferation, invasion and tumor growth and metastasis}

Anguraj Sadanandam ${ }^{1}$, Michelle L.Varney ${ }^{1}$, Michael A. Hollingsworth $^{1}$, Surinder K. Batra ${ }^{1}$, David W. Sretavan $^{2}$, Andreas W. Puschel ${ }^{3}$, Rakesh K.Singh ${ }^{1}$

${ }^{1}$ University of Nebraska Medical Center, Omaha, Nebraska, USA, ${ }^{2}$ University of California San Francisco, California, USA, ${ }^{3}$ Institut fur Allgemeine Zoologie und Genetik, Westfalische Wilhelms-Universitat Munster, Munster, Germany

Pancreatic cancer is the fourth leading cause of cancer death in the United States with an overall 5-year survival of less than $4 \%$. Over the last decade, significant advances have been made in understanding the molecular biology and genetics of the disease. However, other aspects of pancreatic cancer, such as tumor invasion, tumor-stromal interactions and metastasis are poorly understood. Previously, we identified semaphorin 5A (SEMA5A) as a candidate gene for tumor progression and metastasis using in vivo phage display peptide library screening and bioinformatics. We have observed the expression of SEMA5A in pancreatic carcinoma cell lines ( 8 of 10) established from metastases but not primary tumors. All the cell lines examined expressed functional SEMA5A receptor Plexin-B3. Based on the expression pattern of SEMA5A, we examined the role of SEMA5A in pancreatic tumor progression and metastasis by transfecting cell lines, Pancl (nontumorigenic) and AsPC1 (established from ascites) both not expressing SEMA5A, with mammalian expression vector containing SEMA5A cDNA for secreted or membrane-bound forms and their controls. Pancl and AsPC1 SEMA5A transfected cell proliferation in 
different serum concentrations and matrigel invasion were significantly higher $(P<0.05)$ as compared to their control transfected cells. In addition, Panc1 expressing membrane bound SEMA5A underwent more homophilic cellular aggregation compared to its control transfected cells. To recapitulate, in vivo tumor growth was measured by injecting Panc1- and AsPC1-SEMA5A cells subcutaneously into athymic nude mice. Tumor incidence in the Pancl transfected with membrane bound SEMA5A is $100 \%$ as opposed to $0 \%$ in mice injected with transfected cells. Furthermore, tumor growth in mice injected with Panc1- and AsPC1-SEMA5A are significantly higher compared to their controls. Together, these data suggest that SEMA5A expression in pancreatic cancer cells may regulate the phenotypes associated with pancreatic tumor cell growth and metastasis. Therefore, the peptide screened by means of phage-display peptide library may be used as a therapeutic target for metastatic pancreatic cancer cells expressing SEMA5A.

\section{PD 2-6}

\section{Phosphoglucose isomerase signaling enhances colon cancer metastasis}

Soichi Tsutsumi ${ }^{1}$, TatsuoShimura ${ }^{1}$, Takayuki Asao ${ }^{1}$, Hiroyuki Kuwano ${ }^{1}$, Avraham Raz ${ }^{2}$

${ }^{1}$ Department of General Surgical Science (Surgery I), Gunma University Graduate School of Medicine, Gunma, Japan, ${ }^{2}$ Tumor Progression and Metastasis Program, Karmanos Cancer Institute, Wayne State University, USA

Purpose Phosphoglucose isomerase (PGI)/autocrine motility factor (AMF) is a ubiquitous cytosolic enzyme that plays a key role in glycolysis. PGI/AMF is also a multifunctional protein that acts in the extracellular milieu as a potent mitogen/cytokine. Increased expression of AMF/PGI and its receptor has been found in a wide spectrum of malignancies and is associated with cancer progression and metastasis. Recent studies indicated that AMF is induced by hypoxia and enhances the random motility of pancreatic cancer cells. In the present study, the role and regulation of PGI/AMF in the growth and metastasis of colon cancer cells were determined. Experimental design In this study, we also investigated the intracellular signal transduction pathways of PGI in human colon cancer cell lines. We also assessed whether overexpression of PGI in human colon cancer cells enhances the liver metastasis using an orthotopic mouse tumor model. We compared the degree of colocalization of PGI with a chemical marker for hypoxia, pimonidazole by immunostaining. Results Overexpression of PGI leads to constitutive upregulation of glycolysis, even in the presence of oxygen and resistance to extracellular acidosis. Acidification of the microenvironment facilitates tumor metastasis through acid-induced degradation of the extracellular matrix and promotion of angiogenesis. In addition, overexpression of PGI stimulated in vitro invasion of DLD-1 and SW480 human colon cancer cells. In vivo, after orthotopic implantation on the cecum of nude mice, parental and empty vector-transfected DLD-1 cells produced locally relatively small tumors with no evidence of liver metastasis, whereas PGI-transfected DLD-1 cells produced the large tumors and liver metastases. Expression of PGI was presented in the most hypoxic regions that border necrosis in xenograft tumors composed of DLD-1 cell. Expression of AMF protein was examined in paraffin sections from colorectal cancer and control patients by immunohistochemistry. Strong cytoplasmic staining of AMF protein was detected in cancers but was weakly expressed in nontumor tissues. Conclusions The data submitted here show that PGI expression significantly contributes to the aggressive phenotype of human colon cancer and thus may provide a novel prognostic and therapeutic target.

\section{PD 2-7}

\section{High molecular weight-tropomyosins in TGF-b- mediated regulation of invasion and metastasis}

Andrea E. Varga ${ }^{1}$, Qiao Zheng ${ }^{1}$, Nina V. Stourman ${ }^{2}$, Alfiya F. Safina ${ }^{1}$, Andrei V. Bakin ${ }^{1}$

${ }^{1}$ Department of Cancer Genetics, Roswell Park Cancer Institute, Buffalo, New York, USA, ${ }^{2}$ Department of Biochemistry, Vanderbilt University, Nashville, Tennessee, USA

Dynamics of the actin cytoskeleton is critical for tumor cell invasion and metastasis. In normal epithelial cells, transforming growth factor-beta (TGF-b1) induces formation of stable actin filaments called stress fibers, whereas in metastatic cells this response is lost or significantly attenuated. We have shown that induction of stress fibers requires actin-stabilizing proteins: high molecular weight-tropomyosins (HMW-TMs), encoded by TPM1 and TPM2 genes. TGF-b1 up-regulates HMW-TMs in normal cells and suppression of TMs by RNAi reduces stress fibers. Here we investigated the mechanism and biological significance of TPM1 silencing in metastatic cell lines. In metastatic breast cancer cell lines MDA-MB-231, MDA-MB-435 and metastatic colon cell line SW620, mRNA levels of TPM1 were 
significantly reduced compared to normal and tumor non-metastatic cell lines. Treatment with demethylating agent 5-aza-deoxycytidine (5 aza-dC) restored regulation of TPM1 by TGF-b1 with no effect on TPM 2 and MEK-ERK signaling. Cells treated with 5-aza-dC formed stress fibers and showed a reduced motility in response to TGF-b1. Bisulfite sequencing analysis revealed the presence of significant DNA methylation within a $1.8 \mathrm{~kb} 5$-prime-CpG island of the TPMI promoter. To study effects of restoration of TM expression, TM3 isoform encoded by TPM1 was expressed in MDA-MB-231 cells using a Tetracycline-Off (Tet-Off) system. TM3 reduced both migration and invasiveness of tumor cells nearly twofold. This was associated with an increase in stress fibers and cell adhesion to extracellular matrix (ECM). Importantly, TM3 reduced degradation of ECM at focal contacts (invadopodia) via a mechanism involving stress fibers. Cells expressing TM3 were impaired in ability to form lung metastasis after injection in the tail vein in SCID mice. In conclusion, our findings indicate that HMW-TMs are critical for TGF-b1 mediated control of cell motility and invasion. Epigenetic and oncogenic factors downregulating levels of HMW-TMs increase metastatic potential of tumor cells.

\section{PD 2-8}

\section{LIM kinase 1/cofilin pathway is involved in regulating endocytic trafficking of EGF receptor in human breast cancer cells}

Yukio Nishimura ${ }^{1}$, Kiyoko Yoshioka ${ }^{2}$, Ora Bernard ${ }^{3}$, Biborka Bereczky ${ }^{1}$, Kazuyuki Itoh $^{2}$

${ }^{1}$ Graduate School of Pharmaceutical Sciences, Kyushu University, Fukuoka, Japan, ${ }^{2}$ Department of Biology, Osaka Medical Center for Cancer and Cardiovascular Diseases, Japan, ${ }^{3}$ The Walter and Eliza Hall Institute of Medical Research

We have previously shown that overexpression of LIM kinase1 (LIMK1) resulted in a marked retardation of the internalization of the receptor-mediated endocytic tracer, Texas red-labeled epidermal growth factor (EGF) in low-invasive human breast cancer cell MCF-7. We thereby postulate that LIMK1 signaling plays an important role in the regulation of ligand-induced endocytosis of EGF receptor (EGFR) in tumor cells by reorganizing and influencing actin-filament dynamics. In the present study, we further assessed the roles of wild-type LIMK1, a kinase-deficient dominant negative mutant of LIMK1 (DN-LIMK1) and constitutively active, unphosphorylatable cofilin mutant (S3A cofilin) on internalization of EGF-EGFR in MDA-MB-231, a highly invasive human breast cancer cell line. We found a marked delay in the receptor-mediated internalization of Texas red-labeled EGF in the wild-type LIMK1 transfectants. Most of the internalized EGF staining were accumulated within transferrin receptor-positive early endosomes after $30 \mathrm{~min}$ internalization. In contrast, the expression of dominant-negative LIMK1 mutant facilitated the endocytosis of Texas red-EGF, and large amounts of Texas red-EGF staining already reached LIMPII-positive late endosomes/lysosomal vacuoles after $15 \mathrm{~min}$ internalization. We further analyzed the effect of S3A cofilin mutant on EGFR trafficking, and found an efficient delivery of Texas red-EGF into late endosomes/lysosomes at 15-30 $\mathrm{min}$ after internalization. Taken together, these findings implicate that LIMK1 signaling plays a pivotal role in the regulation of EGFR trafficking through the endocytic pathway in invasive breast cancer cells.

\section{PD 2-9}

\section{Variant forms of calponin h1 resistant to actin rearrangement by $\mathrm{PKC}$}

Akemi Sugaya, Naoki Itano, Michiko Takeoka, Shun'ichiro Taniguti

Department of Molecular Oncology, Division of Molecular and Cellular Biology, Institute on Aging and Adaptation, Shinshu University Graduate School of Medicine, Nagano, Japan

Calponin $\mathrm{h} 1$ is an actin-binding protein in smooth muscle cells, known to be involved in the regulation of smooth muscle contraction. We have reported that calponin $\mathrm{h} 1$ suppressed malignant phenotypes, such as cell motility, through its ability to stabilize actin filaments. In this study, we examined the interaction between calponin $\mathrm{h} 1$ and actin using various variant forms of calponin, to further understand the calponin domain responsible for the actin binding. First we observed that the calponin repeat (CNR; tandem repeat like UNC-87 from C. elegans) 1 is also required for the binding activity to actin in addition to hitherto know actin binding site (ABS). Next, we directed attention to the $175 \mathrm{~S}$ and $184 \mathrm{~T}$ in CNR1, which are known as phosphorylation targets by PKC and RhoK. It has been reported that those phosphorylation result in the suppression of calponin hl binding ability to actin. We prepared point-mutated forms of calponin $h 1$, such as S175A and/or T184A, and then transfected those mutants into HeLa cells, and examined the interaction with actin filaments. We observed that the binding of cal- 
ponin to actin was mainly dependent on the 175th amino acid, and S175T mutant was resistant to actin rearrangement by PKC. These results suggested us that the S175T variant calponin will suppress malignant phenotypes more effectively than wild type of calponin.

\section{PD 2-10}

\section{Autotaxin is overexpressed in glioma and contributes to cell motility of glioblastoma cells}

Takamitsu Fujimaki ${ }^{1}$, Takao Yamori ${ }^{2}$, Junken Aoki ${ }^{3}$, Hiroyuki $\mathrm{Arai}^{3}$

${ }^{1}$ Department of Neurosurgery, Teikyo University, Tokyo, Japan, ${ }^{2}$ Division of Molecular Pharmacology, Cancer Chemotherapy Center, Japanese Foundation for Cancer Research, Japan, ${ }^{3}$ Graduate School of Pharmaceutical Sciences, the University of Tokyo, Japan

Autotaxin (ATX) is an enzyme having lysophospholipase activity and isolated originally from melanoma cells. ATX converts lysophosphatidylcholine (LPC) to bioactive lysophosphatidic acid (LPA). Recently, ATX has been shown to have cell motility stimulating activity. This study was conducted to investigate ATX involvement in the biology malignant glioma. ATX protein/mRNA expression was studied in tumor cell lines and surgical specimens. In vitro motility assay was used to examine the effect of ATX. ATX transfected mouse glioma $203 \mathrm{G}$ were also used for in vitro and in vivo experiments. Fifty cell lines from various tumor tissue including colon cancer, lung cancer breast cancer, melanoma and glioma were examined. ATX is highly expressed in most of glioma cell lines but quite few in other tumor cell lines in both protein levels and in mRNA. Glioma cell line which have highest ATX expression and ATX transfected glioma cells showed high motility and migration activity in response to LPA and LPC. This migration was mediated by LPA receptor LPA1 and the activity was completely abolished by LPA1-antagonist Ki16425. Thus it was shown that the ATX contributed to cell motility by autocrine manner. As for surgical specimen, glioblastoma tissue showed high expression of ATX but it was low in other glioma as well as in benign brain tumors. ATX might play some role in invasive nature of glioblastoma.

\section{PD 2-11}

\section{PossibleroleofASGPR in the development of liver metastasis from gastric cancer}

Laura Gomez-Santos ${ }^{1}$, Yoshimi Ohashi ${ }^{1}$, Nobuaki
Higashi $^{1}$, Rika Fukui ${ }^{2}$, Koichi Hirata ${ }^{2}$, Shouji Nakamori $^{3}$, Tatsuro Irimura ${ }^{1}$

${ }^{1}$ Department of Cancer Biology and Molecular Immunology, Faculty of Pharmaceutical Sciences, The University of Tokyo, ${ }^{2}$ Department of Cancer Biology and Molecular Immunology, Faculty of Pharmaceutical Sciences, The University of Tokyo, ${ }^{3}$ Department of Cancer Biology and Molecular Immunology, Faculty of Pharmaceutical Sciences, The University of Tokyo, ${ }^{4}$ Department of Surgery, Sapporo Medical University School of Medicine, ${ }^{5}$ Department of Surgery, Sapporo Medical University School of Medicine, ${ }^{6}$ Department of Surgery and Molecular Medicine, Osaka National Hospital, ${ }^{7}$ Department of Cancer Biology and Molecular Immunology, Faculty of Pharmaceutical Sciences, The University of Tokyo

Background and aim A clinical experiment, which showed that the survival free of liver metastasis and overall survival of gastric cancer patients was improved by continuous intravenously administration of galactose (Gal) (Kosik et al. 1997), supports the hypothesis that hepatic asialoglycoprotein receptor (ASGPR) is involved in the arrest and subsequent growth of gastric cancer cells. We aimed to determine the expression of the specific ligand for ASGPR in the primary tumor and its implication in the metastatic capacity of gastric cancerous cells to the liver. Methods The recombinant protein corresponding to the carbohydrate recognition domain (CRD) of the major subunit of human ASGPR (rhASGPR1) was expressed, purified, biotinylated and used on human gastric cancer sections following histochemical methods. Also, we performed histochemistry by using the plant lectins VVA-B4 and GSL-I, specific for $N$ Acetylgalactosamine (GalNAc) and $\mathrm{Gal}$ and with affinity for cancerous gastric mucosa. The human gastric cancer cell line AZ521 was sorted according to its affinity for rhASGPR1 into the variant cells ; AZHigh3, with high binding, and AZ-Low3, with low binding, which were intrasplenically injected into nude mice to assess their metastatic potential to the liver. Results rhASGPR1 stained the cancerous gastric mucosa with a staining pattern more restricted than VVA-B4 and GSL-I. The mice injected with AZHigh3 cells developed a higher number of metastatic foci and their livers had a higher weight than those treated with AZ-Low3 cells. An in vitro proliferation assay showed that the proliferation rate of AZHigh3 and AZ-Low3 cells on the presence of rhASPGR1 were not significantly different. Discussion We can conclude that the specific ligand for ASGPR is ex- 
pressed by some cell subpopulations in the primary tumor. That expression seems to confer the gastric cancerous cells a higher metastatic capacity to the liver.

\section{PD 2-12}

\section{Differential effects of antioxidants on in vitro invasion, growth and lung metastasis of murine colon cancer cells}

\section{Masaru Ogasawara, Takayuki Matsunaga, Hideyo} Suzuki

Toyama Prefectural Institute for Pharmaceutical Research, Toyama, Japan

Background Cancer metastasis is a major cause of death in cancer patients, and its blockade is believed to enable cancer patients to live longer. Thus, it is important to find promising agents with antimetastatic activity. A variety of antioxidants such as antioxidative vitamins and polyphenols have recently been proposed to be effective in inhibiting cancer metastasis mainly based on the inhibition of tumor invasion, growth and angiogenesis. However, it is unclear which antioxidant has more potent antimetastatic activity. In this study, a comparative study on the inhibitory activities against tumor invasion, growth and experimental lung metastasis among 20 antioxidants was conducted. Methods Experimental lung metastasis assay was performed by inoculation of murine colon 26-L5 cells through a tail vein of mice and tumor nodules on the surface of lungs were determined. Test compounds were administered intraperitoneally 5 consecutive times beginning 3 days before tumor inoculation at a dose of $1 \mu \mathrm{mol}$. Cell invasion was assayed by transwell chambers with filters coated with fibronectin and Matrigel on the lower and upper surfaces, respectively. After 6-h treatment of tumor cells with compounds, cells that had invaded to the lower surfaces were determined colormetrically using crystal biolet. Cell proliferation was determined by WST-1 solution after 48-h treatment of tumor cells with compounds. Free radical scavenging assay was carried out using the stable radical, 1,1-diphenyl-2-picrylhydrazyl. Results Among 20 compounds tested, epigallocatechin gallate (EGCG), gallocatechin gallate and genistein exhibited significant reduction by $77 \%$, $46 \%$ and $44 \%$ in tumor metastasis, respectively. Quercetin showed slight but not statistically significant inhibition. $\alpha$-Tocopherol, $\beta$-carotene and ascorbic acid had no effect. EGCG also inhibited it dose-dependently with $98 \%$ suppression at $2 \mu \mathrm{mol}$, whereas its related compounds, epicatechin gallate and epigallocatechin, did not. EGCG inhibited tumor cell invasion and proliferation significantly, but both the activities were much less effective than those of some of compounds which did not show antimetastatic effect. In addition, not statistically significant relation was observed between the radical scavenging activities of test compounds and their inhibition rates of tumor metastasis. Conclusions These results suggest that EGCG has potential benefit for tumor metastasis inhibition. Its antimetastatic mechanism seemed to be independent of the inhibition of tumor invasion and growth, and the radical scavenging activity.

\section{PD 2-13}

\section{Investigation of a novel metastasis associated gene mag-1}

Yinglin Lu, Huiyun Lin, Xiaogang Tan, Jinqiang Zhang, Yuanji Xu, Huihua Chen, Yan Wang, Gang Liu, Zhiyan $\mathrm{Du}$

Beijing Institute of basic medical sciences, Beijing, P.R. China

Using SSH and DNA microarray technique, 79 ESTs were found expressed two times or more in highly metastatic cell subpopulation PLA801 D than in poorly metastatic cell subpopulation PLA801 C. Both PLA801 $\mathrm{C}$ and PLA801 D were derived from the same parental lung giant cell carcinoma cell line PLA801. EST NO 3 i.e. mag- 1 encoding a polypeptide of 122 amino acids was obtained based on sequencing of the ESTs and sequence data mining in Genbank.

mag-1 was cloned into pET-28a vector and expressed in E. coli BL21 and the antibody of recombinant MAG-1 protein was prepared in rabbit. Using this antibody, a protein band about 45KD was detected in PLA801 D cell line by western blot.

By the bioinformatics analysis, the complete mag- 1 is located at chromosome 4q21.23. Sequence analysis of MAG-1 protein shows that it contains a predicted signal peptide (amino acid 1-29), an acyltransferase domain (amino acid 224-333).

Dot blot hybridization demonstrated that mag- 1 mRNA was ubiquitously expressed in human tissues, including adult tissues, fetal tissues and cancer cell lines. However the levels of mag- 1 mRNA expression in fetal tissues and cancer cell lines were lower than that in adult tissues. MAG-1 protein was detected in serum-free medium of PLA801 C and PLA801D. Therefore it is a secreted protein. Its location is in cytoplasm identified by indirect immuofluorescence. MAG-1 was detected in different cancer cell lines by western blot. 
For a better understanding the function of mag- 1 , a plasmid-based on polymerase U6 promoter system was used to deliver short interfering RNA (siRNA) targeting mag- 1 to the highly metastatic PLA801D cells.

RT-PCR and Western blot examination revealed that mag -1 expressed lower in cells transfected by mag- 1 RNAi than cells stable transfected with mock control. After stable transfected with the RNAi expression vector, cells displayed lower proliferation ability, adhesion ability and migration ability as compared with cells transfected with mock control. FITC-phalloidin staining revealed less actin filament bundles and a fuzzy network of shorter filaments in the mock control, while there were thick and orderly arranged actin filament in the RNAi transfected cell line.

In summary, down regulated mag- 1 had impacts on the metastasis character of PLA-801D cell line in several aspects including proliferation, adhesion, invasion etc. magl may be a novel tumor metastasis associated gene.

\section{PD 3-1}

\section{The Six1 homeoprotein induces epithelial to mesenchymal transition and breast cancer metastasis}

Kimberly L. Christensen ${ }^{1}$, Ricardo D. Coletta ${ }^{2}$, Paul Jedlicka $^{1}$, J. Chuck Harrell ${ }^{1}$, Kathyrn B. Horwitz ${ }^{1}$, Heide L. Ford ${ }^{1}$

${ }^{1}$ University of Colorado Health Sciences Center, Aurora, Coloardo, USA, ${ }^{2}$ University of Campinas Dental School, Piracicaba-SP, Brazil

It is becoming widely accepted that normal development and tumorigenesis are influenced by the same morphogenic processes. Transcription factors that orchestrate developmental organogenesis, such as homeoproteins, can often contribute to tumorigenesis. The Six family of homeoproteins play important roles in development, in part by promoting proliferation, inhibiting apoptosis, and affecting cell migration. Six 1 is overexpressed in many cancers including breast cancer, Wilms'tumor, and alveolar rhabdomyosarcoma, where it affects transformation, proliferation and metastasis. This suggests that misexpression of Six1, after development is complete, may promote tumorigenesis by aberrantly activating transcriptional programs that normally contribute to organogenesis. Here, we show that Six1 expression in MCF12A human mammary epithelial cells induces an epithelial to mesenchymal transition (EMT), including loss of epithelial markers, up-regulation of mesenchymal markers, increased migration and invasion, and decreased cell-matrix adhesion. In this system, expression of Six 1 is transforming, leading to locally invasive tumors in vivo, when grown orthotopically in nude mice. EMTs are critical at many stages of embryogenesis, and similar losses of epithelial characteristics are becoming increasingly linked to local tumor invasion (as is observed with Six1 overexpression in MCF12A cells) and to metastatic dissemination of carcinoma cells. In support of a role for Six 1 in breast cancer metastasis, we have demonstrated that while Six1 overexpression is observed in approximately $50 \%$ of primary breast tumors, it is found in a much higher $90 \%$ of metastatic lesions; and its overexpression correlates significantly with lymph node metastasis $(P<0.05)$, but not with other variables, such as age, histologic type, and clinical stage. In addition, preliminary data from a xenograft model demonstrate that Six 1 overexpression in MCF7 human breast cancer cells induces lymphatic and bone metastases in vivo. Understanding the role of Six1 in breast cancer progression will provide critical insight to the clinical relevance of Six1 overexpression in breast cancer and its potential prognostic and therapeutic significance. As a developmental regulator, Six 1 may provide an ideal drug target for cancers in which it is aberrantly overexpressed, as it is absent or low in most adult tissues, and would inhibit tumorigenesis on multiple fronts, including proliferation and metastasis.

\section{PD 3-2}

\section{Promotion of metastasis in murine melanoma B16 cells by Hgs/GEF-1, and suppression by Hgs/GEF-1 deletion mutants}

\section{Kiyoshi Ogura, Ikuo Kawashima, Tadashi Tai}

The Tokyo Metropolitan Institute of Medical Science, Tokyo, Japan

We previously cloned and characterized GalCer expression factor-1 (GEF-1), a rat ortholog of hepatocyte growth factor (HGF)-regulated tyrosine kinase substrate (Hgs), which was identified as a protein whose phosphorylation is induced by stimulation with HGF. GEF-1 comprises four domains: a zinc-finger $(\mathrm{Z})$, a proline-rich domain $(\mathrm{P})$, a coiled-coil $(\mathrm{C})$, and a proline/glutamine-rich domain (Q). Hgs has been implicated in endosome transports and signal transductions. However, the signal transductions by $\mathrm{Hgs}$ are not clear. We generate therefore canine epithelial MDCK transfectant cells (MDCK/GEF-1cells), which overexpress GEF-1. MDCK/ GEF-1 cells showed GalCer expression, fibroblastic morphological changes, a migratory, an invasive, and a tumorigenic 
phenotype, and expressed non-epithelial cell specific proteins. GEF-1 induce the MDCK cells EpithelialMesenchymal Transition (EMT), in which polarized epithelial cells are converted into motile cells. Domain truncated mutant experiments reveal that C-Q domains of GEF-1 are essential to induce EMT. Q domain truncated mutants (ZPC, PC, C) inhibit EMT by stimulation with $\mathrm{HGF}$, whereas $\mathrm{Q}$ domain had no effect. EMT is a highly conserved and fundamental process that governs morphogenesis. GEF-1 should have important roles in signal transduction pathways for EMT, because Hgs null knockout mice were embryonicall lethal. EMT also has a more sinister role in tumor metastasis. EMT induces indeed local dissemination, invasion, and intravasation of carcinoma cells. Here, we generate murine malignant B16 melanoma transfectants, which overexpress GEF-1 (B16/GEF-1 cells) or $\mathrm{C}$ domain of GEF-1 (B16/C cells). We determine effects of GEF-1 or $\mathrm{C}$ domain on metastasis of the $\mathrm{B} 16$ melanoma cells. In migration assay in vitro with Transwells, B16/GEF-1 cells showed $180 \%$ of migratory activity, but B16/C transfectants cells showed only $1 \%$ of migratory activity, as compared with B16 parent cells. When B16 cells are injected intravenously into $\mathrm{C} 57 \mathrm{BL} / 6$ mice in experimental metastasis assay in vivo, the mean survival time of B16/GEF-1 cells (25 days) and B16/C cells (87 days) was shorter and longer that of the B16 parent cells (42 days), respectively. These findings suggest that $\mathrm{Hgs} / \mathrm{GEF}-1$ also promotes EMT in the B16 melanoma cells, but $\mathrm{C}$ domain of GEF-1 suppresses EMT. Hgs/GEF-1 and its $\mathrm{C}$ domain might therefore be good targets for EMT-blocking drugs, which inhibit metastasis of cancer.

\section{PD 3-3}

\section{Mesenchymal to epithelial transition in the establishment of secondary tumours: insights from a bladder carcinoma progression series}

Elizabeth D. Williams ${ }^{1,2}$, Christine L. Chaffer ${ }^{1,2}$ Samantha R. Oakes ${ }^{3}$, Christopher J. Ormandy ${ }^{3}$, Tony Blick ${ }^{2}$, Erik W. Thompson ${ }^{1,2}$

${ }^{1}$ Bernard O'Brien Institute of Microsurgery, Melbourne, Australia, ${ }^{2}$ Department of Surgery (St. Vincent's Hospital), University of Melbourne, Parkville, Australia, ${ }^{3}$ The Garvan Institute of Medical Research, Darlinghurst, Australia

Tumour metastasis remains the primary cause of morbidity and mortality for cancer sufferers. A better understanding of the molecular process is required to facilitate the devel- opment of new therapeutic strategies. The breakdown of epithelial cell homeostasis leading to aggressive cancer progression has been correlated with the loss of epithelial characteristics and the acquisition of a migratory phenotype. This phenomenon, referred to as epithelial to mesenchymal transition (EMT), has been identified as a crucial event in the development of metastasis. Furthermore, it has been recognized that to form tumours at secondary sites it is likely that the tumour cells must re-activate certain epithelial properties, i.e. undergo a mesenchymal to epithelial transition (MET). We have selected the TSU-Pr1 bladder carcinoma cell line in vivo for increased metastatic ability following intracardiac inoculation, generating a series of 3 related cell lines. Through Affymetrix microarray gene expression analysis, we identified genes that were differentially expressed across these cell lines. A striking feature associated with increasing metastatic ability was an increase in a number of epithelial markers, suggestive of a MET. This was supported by phenotypic changes in the in vitro characteristics of the cells, including altered appearance of the cells from a 'fibroblastic' to an epithelial, cobblestone morphology, decreased migration and invasion abilities, and a diminished ability to form colonies under conditions of anchorage independent growth. Despite the overriding epithelial nature of the metastatic cells, mesenchymal characteristics, such as elevated expression of various matrix metalloprotease family members and E-cadherin repressing transcription factors, were also observed. This supports the notion of tumour cell plasticity and suggests that tumour cells may exhibit a previously undescribed combination of epithelial and mesenchymal traits which is likely to be important in determining metastatic ability. Coordinated alteration in the fibroblast growth factor (FGF)/FGF receptor axis indicated that this pathway may be driving the MET, reminiscent of embryological processes. Indeed, knockdown of the FGF receptor 2, using RNA interference, reversed the epithelial phenotype (appearance and functional assays) of the most metastatic cell line in vitro, an effect that was related to the degree of receptor knockdown. Thus MET at the distant site is likely to be important in the growth of a secondary tumour mass. Understanding how tumour cells use both epithelial and mesenchymal characteristics to disseminate and form secondary tumours is likely to provide potential new targets for intervention.

\section{PD 3-4}

Involvement of epithelial-mesenchymal interaction in cell invasiveness of oral squamous cell carcinoma

Hirokazu Nakahara $^{1}$, Yudai Matsuoka ${ }^{1}$, Tomohiro Otani $^{2}$, Mikihiko Kogo ${ }^{1}$ 
${ }^{1}$ The First Department of Oral and Maxillofacial Surgery, Osaka University Graduate School of Dentistry, Osaka, Japan, ${ }^{2}$ Kinki University Medical School, Department of Oral and Maxillofacial Surgery, Japan

The migration of tumor cells through the extracellular matrix is thought to be a significant step of the metastasis process. Several matrix metalloproteinases (MMPs) have been shown to play an important role in the invasion and metastasis of oral squamous cell carcinoma (SCC). We have used fibronectin degradation/invasion assay and analyzed the molecular invasion mechanism of SCC. Although gene expression or protein expression peculiar to a cancer cell is important, it is also important for cell kinetics with interaction epithelial cells and mesenchymal cells. Therefore mixed culture with cancer cells and fibroblasts were done based on the fibronectin degradation/invasion assay and observed cell kinetics of the cancer cell. Three cell lines, OSC-20 cells, OSC-19 cells, and HOC313 cells which showed an mode of invasion Grade 3, Grade 4C, and Grade 4D in a YamamotoKohama classification (Y-K classification) with a human oral SCC were used. Three kinds of SCC cells enhanced the invasion ability by what it incubated with fibroblasts than it incubated alone. Enhancement of invasion ability was found in the HOC 313 cell which did not show so high invasiveness by the tumor cells were plated alone in the fibronectin degradation/invasion assay. On the other hand, we were able to inhibit the invasion ability by down-regulated proteases expression (MT1-MMP, MMP-2, MMP-9) with small interfering RNA of the cancer cell side or fibroblast side.

\section{PD 3-5}

\section{Role of ADAM28 in tumor cell adhesion to endothelial cells and transendothelial migration through interaction with P-selectin glycoprotein ligand-1}

Shimoda Masayuki ${ }^{1}$, Hashimoto Gakuji ${ }^{1}$, Mochizuki Satsuki ${ }^{1}$, Ikeda Eiji ${ }^{1}$, Nagai Norihiro ${ }^{2}$, Ishida Susumu ${ }^{2}$, Okada Yasunori ${ }^{1}$

${ }^{1}$ Department of Pathology, Keio University School of Medicine, Tokyo, Japan, ${ }^{2}$ Department of Ophthalmology, Keio University School of Medicine, Tokyo, Japan

ADAM28 is a member of the ADAM (a disintegrin and metalloproteinase) gene family, which are characterized by the disintegrin and metalloproteinase domains, and is composed of two alternative forms, i.e., prototype membrane-anchored form (ADAM28m) and secreted form (ADAM28s). We have recently reported that
ADAM28 is highly expressed in non-small cell lung carcinomas with correlations to the carcinoma cell proliferation and lymph node metastases. To analyze the possible involvement of ADAM28 in tumor cell metastasis, we screened binding molecules to ADAM28s by the yeast two-hybrid system and identified P-selectin glycoprotein ligand (PSGL-1). Yeast two-hybrid assays and binding assays demonstrated that the disintegrinlike domain of ADAM28s interacts with the extracellular decamer repeats of PSGL-1. This specific binding between ADAM28s and PSGL-1 on the cell membranes was further determined by binding assays of the domain-specific recombinant ADAM28s species using PSGL-1 stable transfectants and PSGL-1-expressing cell lines (human promyelocytic cell line HL-60 cells and Tlymphoma cell line Jurkat cells), and co-localization and co-immunoprecipitation of the molecules in these cells incubated with recombinant ADAM28s species. PSGL1 is a major P-selectin ligand expressed on leukocytes, leukemia cells and prostate carcinoma cells, and mediates the adhesion of these cells on the vascular endothelium. Cell adhesion assays demonstrated that incubation of PSGL-1 expressing tumor cells (HL-60 cells and prostate cancer cell line MDA PCa 2b cells) with recombinant ADAM28s enhances the binding to P-selectin-coated wells and thrombin-stimulated human umbilical vein endothelial cells. However, the proteinase activity of ADAM28s was not required for the binding, since both proADAM28s and active ADAM28s exhibited the effect, which was not abrogated by treatment with a synthetic ADAM inhibitor. The increased binding was inhibited by treatment with the antibody specific to the decamer repeats of PSGL-1. Adhesion of intravenously injected HL-60 cells to the endothelial cells of alveolar capillaries and their migration into alveolar spaces were enhanced by incubation of HL-60 cells with ADAM28s in an experimental mouse model. These data demonstrate that ADAM28s enhances PSGL-1/P-selectin-mediated tumor cell adhesion to endothelial cells and transendothelial migration through the interaction with PSGL-1 on tumor cells, suggesting the possibility that ADAM28 may be an important element in PSGL$1 / \mathrm{P}$-selectin mediated tumor cell metastasis.

\section{PD 3-6}

\section{Metastatic human colorectal cancer cells adhere preferentially to human endothelial cells from the liver}

David Canovas, John L. Burn, David Mangnall, Ali W. Majeed, Nigel N. Bird

Liver Research Group, Royal Hallamshire Hospital, 
University of Sheffield, Sheffield, UK

Introduction Liver metastasis develops in about $50 \%$ of patients with colorectal cancer and is the leading cause of cancer death in this disease. To establish metastasis in the target organ, circulating tumour cells have to detach from the primary tumour and interact with the endothelial cells in the sinusoids of the liver. However, it is not known whether colorectal cancer cells adhere to the liver because it is the first organ they reach or whether there is any specific interaction between colorectal cancer cells and endothelial cells in the liver. It has been suggested that endothelial cells from large and small vessels may show differences in cell surface molecules related to their origin and the treatment of endothelial cells from different organs with same stimuli induces different expression patterns of adhesion molecules. Aim To study the adhesion of a metastatic colorectal cancer line to endothelial cells from different sources following stimulation with IL-1 $\beta$. Material and methods Three different types of endothelial cells were used in the study. One from large vessel, HUVEC (umbilical cord; $n=5$ ), one from microvascular vessels, HuDMEC (dermal; $n=5$ ), and one from liver sinusoids, HuLiSEC $(n=6)$, were treated with IL-1 $\beta$ for $4 \mathrm{~h}$ at different concentrations. Afterwards, CFDASE labelled colorectal cancer cell lines, HT-29 and Colo 741, and cervix cancer cell line, HeLa, were placed over the endothelial cells and left for $1 \mathrm{~h}$. Non-adhered cells were removed by washing three times with PBS and cells were fixed with formaldehyde. Fluorescence was measured at $492-517 \mathrm{~nm}$ (excitation/emission peaks). Results The results show that the adhesion of HT29 to IL$1 \beta$ stimulated HuLiSEC was significantly increased $(P<0.05)$, giving a dose-response curve peaking at $10 \mathrm{ng} / \mathrm{ml}$ IL-1 $\beta$ treatment. Moreover, HT-29 adhesion to IL-1 $\beta$ stimulated HuLiSEC was significantly $(P<0.05)$ higher than to HuDMEC and HUVEC. Finally, adhesion of HT-29 was significantly higher than the adhesion of Colo 741 and HeLa to IL-1 $\beta$ stimulated HuLiSEC. Conclusions HT-29 cells adhere preferentially to stimulated sinusoidal endothelial cells compared to those from other sources. This may partially explain the frequency with which liver metastasis occurs in colorectal cancer.

\section{PD 3-7}

Highly invasive human pancreatic carcinoma cells adhere to endothelial tri-cellular corners and increase endothelial permeability

Toshiyuki Tanaka ${ }^{1}$, Kei Nakai ${ }^{1,2}$, Toshiyuki Murai ${ }^{1}$, Nobuyuki Ohguro $^{2}$, Yasuo Tano ${ }^{2}$, Masayuki Miyasaka ${ }^{1}$
${ }^{1}$ Laboratory of Immunodynamics, Department of Microbiology \& Immunology, Osaka University Graduate School of Medicine, Osaka, Japan, ${ }^{2}$ Department of Ophthalmology, Osaka University Graduate School of Medicine, Osaka, Japan

Although adhesive interactions between metastasizing cancer cells and vascular endothelial cells are critical in hematogenous metastasis, the early molecular events of the cancer-endothelial interaction remain largely obscure. Here we investigated the functional impact of cancer cells on endothelial permeability. We examined the binding of human pancreatic carcinoma cell lines MIA PaCa-2, PANC1, and PSN-1 to a human umbilical vein endothelial cell (HUVEC) monolayer and the subsequent changes in the transendothelial electronic resistance (TEER) of the HUVECs. We found that MIA PaCa-2 and PANC-1 cells preferentially bound the tri-cellular corners of HUVECs and induced a rapid and irreversible reduction of TEER. The reduction of HUVEC TEER was associated with the focal disengagement of endothelial junctional adhesion molecules VE-cadherin and CD31. Blocking monoclonal antibodies to integrin $\beta 1, \mathrm{CD} 44$, or CD9 affected neither the MIA PaCa-2 cell binding to HUVECs nor the reduction of TEER. Specific inhibitors for metalloproteinases, tyrosine-kinases, and lipoxygenases, and a neutralizing anti-VEGF antibody failed to affect the MIA PaCa-2-induced reduction of HUVEC TEER, whereas treatment of the cells with paraformaldehyde or cytochalasin $\mathrm{B}$ abrogated the TEER reduction. These findings indicate that the MIA PaCa-2 cells bind selectively to endothelial tri-cellular corners, triggering a reduction of HUVEC TEER, which requires the active metabolism and intact actin cytoskeleton of the carcinoma cells, and is apparently unrelated to previously described cell-adhesion and soluble factor pathways involved in cancer-endothelial-cell interactions. Our data indicate a novel cell-contact-dependent mechanism for the cancer-cell-mediated breakdown of endothelial barrier functions, which may be important in hematogenous cancer metastasis.

\section{PD 3-8}

Extracellular matrix: a gatekeeper in the transition from dormancy to metastasis

Jeffrey E. Green ${ }^{1}$, Sylvain V. Costes ${ }^{2}$, Edward H. Cho ${ }^{3}$, Anil K. Kamaraju ${ }^{1}$, Mark Hoenorhoff ${ }^{1}$, Stephen Lockett $^{3}$, Hynda Kleinman ${ }^{4}$, Ann F. Chambers ${ }^{5}$, Dalit Barkan $^{1}$

${ }^{1}$ Laboratory of Cell Regulation and Carcinogenesis, 
NCI, NIH, Bethesda, MD, USA, ${ }^{2}$ Cancer Biology Department, Lawrence Berkeley National Laboratory, Cyclotron Road, Berkeley, CA USA, ${ }^{3}$ Image Analysis Laboratory, SAIC-Frederick, National Cancer Institute, Fredrick, MD USA, ${ }^{4}$ Craniofacial Developmental Biology and Regeneration Branch, National Institute of Dental and Craniofacial Research, NIH, Bethesda, MD USA, ${ }^{5}$ London Regional Cancer Program, 790 Commissioners Road East, London, Ontario, Canada

Late-stage metastatic breast cancer often develops from tumors cells that have seeded metastatic sites, resist conventional therapies, and remain dormant for many years. Dissecting the mechanisms involved in the transition from dormancy to metastatic growth has been hampered by the lack of available model systems. We established a novel in vitro system, initially based on the only available and characterized dormant (D2.0R cells) and metastatic (D2A1) breast cancer cell line system. Upon culturing the D2.0R cells in a 3-dimensional Cultrex, the cells remain quiescent for 14 days. The quiescence was associated with expression of the negative cell-cycle regulators p27 and p16. In contrast, the D2A1 cells remain transiently quiescent for 4-6 days in the 3D system and subsequently proliferated, correlating to the in vivo characteristics of these cells. Importantly, this system is shown to be predictive for the in vivo dormant or metastatic behavior of several tumor cell lines. By Utilizing this system, we demonstrated that the transition of metastatic cells from quiescence to proliferation requires cells to adhere to the matrix, activating myosin light chain phosphorylation by myosin light chain kinase (MLCK), leading to formation of actin stress fibers, whereas dormant cells did not form such fibers. Blocking MLCK activity or its expression, inhibited this transition. Interestingly alterations in the ECM induced transition of D2.0R cells from a persistent dormant state to a proliferative state, followed by actin stress fiber formation. Furthermore, we show that the transition from dormancy to growth is reversible. When selected clonal proliferating D2.OR cells are placed on fresh 3D Cultrex system, the cells reverted to a dormant state; similarly cell lines derived from a metastatic D2.0R lung lesion, reverted to a quiescent state, when placed into the $3 \mathrm{D}$ system. Therefore, our results suggest that epigenetic events lead to the transition from dormancy to growth. In summary we show that metastatic cell interactions with the ECM and its remodeling may be a critical factor for the long-term maintenance of tumor cell dormancy and the eventual emergence of cell growth into clinically significant metastases.

\section{PD 3-9}

\section{Molecular mechanism of nuclear import of galectin-3}

Susumu Nakahara ${ }^{1,2}$, Hidenori Inohara ${ }^{2}$, Avraham Raz $^{3}$, Yoshiharu Sakata ${ }^{1}$

${ }^{1}$ Department of Otolaryngology, Suita Municipal Hospital, Osaka, Japan, ${ }^{2}$ Department of Otolaryngology and Sensory Organ Surgery, Osaka University Graduate School of Medicine, Osaka, Japan, ${ }^{3}$ Tumor Progression and Metastasis Program, Karmanos Cancer Institute, Detroit, USA

Galectin-3 (Gal-3), a $\beta$-galactoside-binding protein, was shown to be involved in several nuclear dependent functions including up-regulation of transcriptional factors and cell cycle regulation. Gal-3 compartmentalization in the nucleus versus the cytoplasm affects the malignant phenotype of various cancers. However, to date the detailed mechanism by which Gal-3 translocates into the nucleus remains unknown. Thus, we have constructed and expressed a variety of fusion proteins containing deletion mutants of Gal-3 fused with enhanced green fluorescent protein (EGFP) and searched for the Gal-3 sequence motifs essential for its nuclear localization. In addition, a digitonin-permeabilized cellfree transport assay was employed to directly examine the mechanism of Gal-3 nuclear import. Here, we report that partial deletions of the C-terminal region (114-250) of the human Gal-3, significantly decreases its nuclear translocation, while a Gal-3 derived peptide (1-115) was transported to the nuclei of both transfected COS-7 cells. The nuclear import assay revealed that there are at least two independent nuclear pathways for shuttling Gal-3 into the nucleus: a passive diffusion and an active transport. This is the first report providing direct evidence for the nuclear import mechanisms of Gal-3, and suggests that Gal-3 nuclear translocation is governed by dual pathways while the cytoplasmic/nuclear distribution may be regulated by a multiple processes including cytoplasmic anchorage, nuclear retention and or nuclear export. These results may lead to the development of a therapeutic modality aiming at abrogating Gal-3 translocation into the nucleus and thus hampering its activity during cancer progression and metastasis.

\section{PD 3-10}

\section{Implication of Galectinin Wnt signaling pathway}

Tatsuo Shimura ${ }^{1,2}$, Souichi Tsutsumi ${ }^{1}$, Taketoshi Suehiro ${ }^{1}$, Hideki Suzuki ${ }^{1}$, Avraham Raz ${ }^{2}$, Hiroyuki Kuwano ${ }^{1}$ 
${ }^{1}$ Department of General Surgical Science (Surgery I), Gunma University Graduate School of Medicine, Gunma, Japan, ${ }^{2}$ Karmanos Cancer Institute, Wayne State University, Detroit, MI, USA

Galectin-3 (gal-3) a pleiotrophic protein is an important regulator of tumor metastasis, which like $\beta$-Catenin, shuttles between the nucleus and the cytosol in a phosphorylation-dependent manner. We report herein that $\beta$-catenin stimulation of cyclin D1 and c-myc expression is gal-3-dependent. Gal-3 binds to $\beta$ catenin/ Tcf complex, co-localizes with $\beta$ catenin in the nucleus and induces the transcriptional activity of Tcf- 4 as determined by the TOP/FOPFLASH reporter system. We have identified the $\beta$ catenin-gal-3 binding sequences, which are in the $\mathrm{N}$ - and $\mathrm{C}$-terminus of the proteins encompassing amino acid residues 1-131 and 143-250, respectively. These data indicate that gal-3 is a novel binding partner for $\beta$ catenin involved in the regulation of $\mathrm{Wnt} / \beta$ catenin signaling pathway. Analysis of the human gal-3 sequence reveled a structural similarly to $\beta$ catenin as it also contains the consensus sequence (S92XXXS96) for glycogen synthase kinase (GSK) $3 \beta$ phosphorylation, and can serve as its substrate. In addition, Axin a regulator protein of Wnt that complexes with $\beta$ catenin also binds gal-3 using the same sequence motif identified here by a deletion mutant analysis. The data presented here give credence to the suggestion that gal-3 is a key regulator in the Wnt/ $\beta$ catenin signaling pathway and highlight the functional similarities between gal-3 and $\beta$-catenin.

\section{PD 3-11}

\section{Maoto, a Kampo medicine, suppresses the motility and metastatic potential of highly metastatic osteosarcoma cells}

Sumiko Hyuga ${ }^{1}$, Masashi Hyuga ${ }^{2}$, Hayao Nakanishi ${ }^{3}$, Setsuko Sekita ${ }^{4}$, Tetsuro Oikawa ${ }^{1}$, Toshihiko Hanawa ${ }^{1}$

${ }^{1}$ Department of Clinical Research, Oriental Medicine Research Center, The Kitasato Institute, Japan, ${ }^{2}$ Division of Biological Chemistry and Biologicals, National Institute of Health Sciences, Japan, ${ }^{3}$ Division of Oncological Pathology, Aichi Cancer Center Research Institute, Japan, ${ }^{4}$ Faculty of Pharmaceutical Sciences at Kagawa Campus, Tokushima Bunri University, Japan

Kampo medicines have been widely used as an adjunctive therapy following surgery, radiation therapy, or chemotherapy for cancer in Japan. Recently, Kampo medicines have been reported to suppress cancer metastasis through activation of the immune system.
However, it remains to be clarified whether Kampo medicines are directly involved in the metastatic process. In the present study, we focused on the effects of Kampo medicines on cancer cell motility, which is closely associated with invasion into the surrounding stroma, intravasation, and extravasation in metastatic processes. In our motility assay, mouse sera (MS) obtained from mice given the Kampo medicine were used, since Kampo medicines are generally administered orally and the MS is assumed to contain both the original compounds of the administered medicine and their metabolites. The motility of FBJ-LL cells, a highly metastatic osteosarcoma cell line, was significantly reduced by MS obtained from mice given maoto, but no significant change was observed in the presence of MS obtained from mice given juzentaihoto or hochuekkito. Normal MS-induced cell motility was also reduced by addition of maoto, suggesting that the original compound (s) in maoto exhibits the reducing activity. Ephedra herb, an ingredient in maoto, inhibited cell motility, and its inhibitory effect was as large as that of maoto, indicating that this herb plays a critical role in the inhibition of cancer cell motility. Ephedrine is a principle component of Ephedra herb, but its effect on cancer cell motility has not been determined. We therefore examined the effect of ephedrine on cancer cell motility. Ephedrine did not inhibit the cancer cell motility and maoto(-), which includes Ephedra americana containing no ephedrine, inhibited the motility to the same degree as maoto. The results thus indicated that ephedrine has no effects on cancer cell motility. We also examined the effect of maoto on spontaneous metastasis in tumor-bearing mice. FBJ-LL cells were subcutaneously transplanted into the thighs of mice, who were then administered maoto, juzentaihoto, or water ad libitum. The mice were sacrificed at 4-5 weeks and metastatic nodules in their livers were counted. The number of nodules was significantly reduced by oral administration of maoto, but not by oral administration of juzentaihoto. These results suggest that maoto inhibits cancer metastasis through the suppression of cancer cell motility and suggests that this agent may be a candidate for a novel inhibitor of metastasis.

\section{PD 3-12}

Galectin-3 regulates mitochondrial stability and anti-apoptotic function in response to anti-cancer drug in prostate cancer

Tomoharu Fukumori ${ }^{1}$, Natsuo Oka ${ }^{1}$, Ryoichi Nakanishi ${ }^{1}$, Kunihisa Yamaguchi ${ }^{1}$, Ryuichi Taue ${ }^{1}$, 
Hiroyoshi Nakatsuji ${ }^{1}$, Tomoteru Kishimoto ${ }^{1}$, Hirofumi Izaki $^{1}$, Masayuki Takahashi ${ }^{1}$, Masa-aki Nishitani ${ }^{1}$, Avraham Raz ${ }^{2}$, Hiro-omi Kanayama ${ }^{1}$

${ }^{1}$ Department of Urology, The University of Tokushima Graduate School of Health Biosciences, Japan, ${ }^{2}$ Tumor Progression and Metastasis Program, Karmanos Cancer Institute, Wayne State University, Detroit, USA

Prostate cancer is one of the malignant tumors, which exhibit resistance to anticancer drugs, at least in part due to enhanced anti-apoptotic mechanisms. Therefore, the understanding of such mechanisms should improve the design of chemotherapy against prostate cancer. Galectin-3, a multifunctional oncogenic protein involved in the regulation of tumor proliferation, angiogenesis, and apoptosis has demonstrated antiapoptotic effects in certain cell types. Here, we show that expression of exogenous galectin-3 in human prostate cancer LNCaP cells, which do not express galectin-3 constitutively, inhibits anticancer drug-induced apoptosis by stabilizing the mitochondria. Thus, galectin-3 negative cells showed $66.31 \%$ apoptosis after treatment with $50 \mu \mathrm{M}$ cis diamminedichloroplatinum for $48 \mathrm{~h}$, whereas two clones of galectin-3 expressing cells show only $2.92 \%$ and $1.42 \%$ apoptotic cells. Similarly, galectin-3 negative cells showed $43.8 \%$ apoptosis after treatment with $300 \mu \mathrm{M}$ etoposide for $48 \mathrm{~h}$, whereas only $15.38 \%$ and $14.51 \%$ of galectin-3 expressing LNCaP cells were apoptotic. The expression of galectin-3 stimulated phosphorylation of Ser 112 of Bad and downregulated Bad expression after treatment with CDDP. Galectin-3 also inhibited mitochondrial depolarization and damage after translocation from nuclei to cytoplasm, resulting in inhibition of cytochrome c release and caspase- 3 activation. These findings indicate that galectin-3 inhibits anticancer druginduced apoptosis through regulation of Bad protein and suppression of the mitochondrial apoptosis pathway. Therefore, targeting galectin-3 could improve the efficacy of anticancer drug chemotherapy in prostate cancer.

\section{PD 3-13}

\section{Prevention of metastasis by galectin-9: mechanism analysis of anti-metastatic function}

Atsuya Nobumoto ${ }^{1}$, Keisuke Takeshita ${ }^{1}$, Keiko Nagahara $^{2}$, Toshiro Niki ${ }^{1}$, Nozomu Nishi ${ }^{1,3}$, Akira Yamauchi $^{1,4}$, Mitsuomi Hirashima ${ }^{1,2}$

${ }^{1}$ GalPharma Co. Ltd., ${ }^{2}$ Department of Immunology and Immunopathology, Kagawa University, Kagawa,
Japan, ${ }^{3}$ Department of Endocrinology, Kagawa University, Kagawa, Japan, ${ }^{4}$ Department of Cell Regulation, Kagawa University, Kagawa, Japan

Background and purpose Galectin-9 (Gal-9) exhibits a variety of biological functions, including aggregation, adhesion, and apoptosis of cancer cells, and maturation of dendritic cells. Furthermore, we recently reported the inverse correlation between Gal-9 expression and distant metastasis in breast cancer and malignant melanoma. The purpose of this study is to investigate the inhibitory effect of galectin-9 on metastasis of tumor cells, and to elucidate its mechanism. Materials and methods (a) Experimental lung metastasis model of mouse with B16F10 melanoma cells was used to examine the effect of intravenous Gal-9 administration or transfection. (b) We analyzed cell surface adhesion molecules and ligand binding involving in cancer metastasis in the presence or absence of Gal-9. We also examined the effects of Gal-9 on adhesion of B16F10 cells to extra-cellular matrices, such as collagen type I, collagen type IV, fibronectin, laminin, and vitronectin in vitro. Results and discussion (a) Thirty microgram of intravenously administrated Gal-9 significantly reduced the number of metastatic colonies in lung. We prepared two kinds of Gal-9 transfectants from B16F10 cells, those with or without signal peptides. Gal-9 transfectants with signal peptides more secrets Gal-9 and less metastasized than those without signal peptides. These data indicate that extra -cellular Gal-9 probably plays a critical role in the metastasis inhibition. (b) Expression analysis of Integlin family shows that Integlin 1, 4, and V were highly expressed on B16 F10 cells, and Gal-9 significantly inhibits the binding of their ligands in vitro, suggesting that Gal-9 inhibits the adhesion of cancer cells to vascular endothelium by blocking the binding to adhesion molecules. Furthermore, we found that Gal-9 suppresses the binding of B16F10cells to extracellular matrices suggesting that Gal-9 also inhibits the invasion of cancer cells. Conclusion These data suggest the possibility of clinical use of Gal-9 by intravenous administration or through the development of gene therapy.

\section{PD 4-1}

\section{Recombinant human fibroblast growth factor-2 stimulates expression of endogenous vascular endothelial growth factor to enhance the growth and metastasis of B 16-BL 6 mouse melanoma cells}

Satoshi Tsunoda ${ }^{1,2}$, Toshiyuki Nakamura ${ }^{2}$, Hiroaki Sakurai ${ }^{1}$, Ikuo Saiki ${ }^{1}$

${ }^{1}$ Division of Pathogenic Biochemistry, Department of 
Bioscience, Institute of Natural Medicine, University of Toyama, Toyama, Japan, ${ }^{2}$ Safety Research Department, Central Research Laboratories, Kaken Pharmaceutical Co., Ltd., Shizuoka, Japan

Fibroblast growth factor-2 (FGF-2) has been recognized an angiogenic growth factor and associated with melanoma growth. We investigated the effects of exogenous recombinant human FGF-2 (rhFGF-2) on the growth and metastasis of B16-BL6 mouse melanoma (B16-BL6) cells. In 1st experiment, to investigate the direct effects of rhFGF-2 on the B 16-BL6 cells in vitro, we evaluated the intracellular signaling pathway, the cellular proliferation and the mRNA expression of endogenous vascular endothelial growth factor (VEGF) after rhFGF-2 stimulation. Exogenous rhFGF-2 induced the tyrosine phosphorylation in FGF receptor (FGFR) and activated the Erk1/2 and Akt, which is almost identical to the signal transduction pathway after the FGF-2 stimulation of FGFR in normal cells. In contract, rhFGF-2 did not enhance the cellular proliferation and level of endogenous VEGFA mRNA. These results suggest that exogenous rhFGF-2 has little potential for the stimulation of the cellular proliferation and endogenous VEGFA mRNA synthesis, even though it activates directly intracellular signaling pathway via mouse FGFR. In 2nd experiment, to investigate the effects of rhFGF-2 on the growth and metastasis of B16-BL6 cells in vivo, we injected rhFGF-2 into the tumor tissue after the inoculation of C57BL6 mice in footpad or dorsal subcutaneous tissue (s.c). Exogenous rhFGF-2 accelerated tumor-induced angiogenesis as well as increased tumor growth, lymph node and lung metastasis. These effects of rhFGF-2 were almost completely inhibited by local injection of neutralizing antibody against VEGFA, which is comparable to the effect of prednisolone. After the injections of rhFGF-2 into s.c at a site remote from the primary tumor, rhFGF-2 was ineffective against tumor growth and lung metastasis. Since exogenous rhFGF-2 did not stimulate directly the cellular proliferation and endogenous VEGFA mRNA expression in vitro, these results suggest that exogenous rhFGF-2 stimulates the endogenous VEGFA synthesis derived from normal cells in host tissue and accelerates the tumor-induced angiogenesis, which play an important role in the enhancing effect of exogenous rhFGF-2 on the tumor growth and metastasis. In addition, these enhancing effects may be observed only when rhFGF-2 was directly injected into the tumor tissue. In the present study, we demonstrate that exogenous rhFGF-2 stimulates endogenous VEGFA synthesis in host cells following the modulation of tumor microenvironment, which triggers the accelera- tion of tumor-induced angiogenesis and enhances subsequently tumor growth and metastasis of B16-BL6 cells.

\section{PD 4-2}

FGF2 binding, signaling, and angiogenesis are modulated by heparanase in metastatic melanoma models

Dario Marchetti, Jane Reiland, Doty Kempf, Madhuchhand A. Roy, Yvonne Denkins

CBS Department, LSU-Baton Rouge, Baton Rouge, LA, USA

Heparanase (HPSE) and fibroblast growth factor-2 (FGF2) are critical regulators of melanoma angiogenesis and metastasis. Elevated HPSE expression contributes to melanoma progression; however, further augmentation of HPSE presence can inhibit tumorigenicity. HPSE enzymatically cleaves heparan sulfate glycosaminoglycan chains (HS) from proteoglycans. HS acts as both lowaffinity FGF2 receptors and co-receptors in the formation of high-affinity FGF2 receptors. We have investigated HPSE ability to modulate FGF2 activity through HS remodeling. Extensive HPSE degradation of human metastatic melanoma cells $(70 \mathrm{~W})$ inhibited FGF2 binding. Unexpectedly, treatment of $70 \mathrm{~W}$ cells with low HPSE concentrations enhanced FGF2 binding. In addition, HPSE-unexposed cells did not phosphorylate extracellular signal-related kinase (ERK) or focal adhesion kinase (FAK) in response to FGF2. Conversely, in cells treated with HPSE, FGF2 stimulated ERK and FAK phosphorylation. Second, presence of soluble HPSE-degraded HS enhanced FGF2 binding and ERK phosphorylation at low HS concentrations. Higher concentrations of soluble HS inhibited FGF2 binding but FGF2 signaling through ERK remained enhanced. Soluble HS were unable to support FGF2-stimulated FAK phosphorylation irrespective of HPSE treatment. Finally, cell exposure to HPSE or to HPSE-degraded HS modulated FGF2-induced angiogenesis in melanoma. In conclusion, these effects suggest relevant mechanisms for HPSE modulation melanoma growth factor responsiveness and tumorigenicity.

\section{PD 4-3}

Hyperproduction of hyaluronan in neu-induced mammary tumor accelerates angiogenesis through stromal cell recruitment: possible involvement of PG-M/versican 
Hiroshi Koyama ${ }^{1,2}$, Zenzo Isogai ${ }^{3}$, Masahiko Yoneda ${ }^{4}$, Minoru Fujimori ${ }^{2}$, Jun Amano ${ }^{2}$, Reiji Kannagi ${ }^{5,6}$, KojiKimata $^{7}$, Shun'ichiro Taniguchi ${ }^{1}$, Naoki Itano ${ }^{1,6}$

${ }^{1}$ Department of Molecular Oncology, Division of Molecular and Cellular Biology, Institute on Aging and Adaptation, Shinshu University Graduate School of Medicine, Japan, ${ }^{2}$ Department of Surgery, Shinshu University School of Medicine, Japan, ${ }^{3}$ National Center for Geriatrics and Gerontology, Japan, ${ }^{4}$ Aichi Prefectural College of Nursing and Health, Japan, ${ }^{5}$ Program of Molecular Pathology, Aichi Cancer Center, Research Institute, Japan, ${ }^{6}$ Core Research for Evolutional Science and Technology, Japan Science and Technology Corporation, Japan, ${ }^{7}$ Institute for Molecular Science of Medicine, Aichi Medical University, Japan

Overproduction and accumulation of hyaluronan in the most invasive breast cancers are well documented by extensive clinical evidence. In an effort to simulate the overproduction of hyaluronan observed in malignant human breast cancer, we generated transgenic mice that express murine hyaluronan synthase2 (Has2) in a Cremediated recombination-dependent manner. The Has2 conditional transgenic mice were then crossed with mice of the MMTV-Neu mammary tumor model. By expressing Cre recombinase under the control of MMTV promoter, the double transgenic mice carrying Has2 and Neu transgenes exhibited overproduction of hyaluronan and its accumulation in mammary tumors. When we examined the early stages of tumor development, the incidence of mammary tumor was significantly greater and growth faster in Has2 overexpressing mice than in the control mice. Histological observations revealed that the forced expression of Has 2 enhanced the recruitment of stromal cells into mammary tumor compared to the control mice. Notably, many microvessels were observed in the mammary tumors of Has2 overexpressing mice after immunohistochemical staining with an antibody specific for CD31. The size distribution of the microvessels was then analyzed by plotting the number of microvessels versus their vascular size. In the Has 2 overexpressing mice, the number of tumor microvessels with a size of $\sim 1,000 \mu \mathrm{m}^{2}$ was 2 -fold greater than in the control mice, suggesting that the acceleration of tumor incidence and growth are partly due to the accelerated neovascularization. By real-time quantitative RT-PCR, the expression of basic FGF and SDF-1 alpha/CXCL12, both of which are stromaderived factors capable of angiogenic stimulation, was 2 -fold increase in the Has 2 overexpressing tumor compared to the controls. To reveal the molecular basis of hyaluronan-mediated neovascularization, various hyal- uronan samples were examined for their ability to potentiate in vivo angiogenesis. In a Matrigel plug assay, bFGF-induced neovascularization was elevated in the presence of either hyaluronan oligosaccharide or a hyaluronan aggregate containing the large chondroitin sulfate proteoglycan, versican. Administration of hyaluronan-versican aggregates, but not native hyaluronan alone, promoted angiogenesis and also stromal cell invasion. Interestingly, the exogenous versican was predominantly incorporated and accumulated into hyaluronan extracellular matrix surrounding stromal cells. Taken together, these results suggest that hyaluronan-versican extracellular matrix accelerates tumor angiogenesis through stromal cell recruitment.

\section{PD 4-4}

\section{TGF-b signaling in tumor angiogenesis and metastasis}

Andrei Bakin, Alfiya Safina, Joseph Spernyak, Richard Mazurchuck

\section{Roswell Park Cancer Institute, Buffalo, USA}

The spread of cancer to distant sites (metastasis) is the main cause of mortality of cancer patients. Prevention of development and treatment of metastatic cancers remain important tasks for cancer medicine. Transforming growth factor beta 1 (TGF-b1) is a potent tumor suppressor, but, paradoxically, TGF-b1 promotes tumor spreading in advanced cancers. Given the dual function of TGF-b1, it is important to develop therapies that suppress oncogenic function of TGF-b and enhance tumor suppressor function. Here we investigated the specific components of the TGF-b pathway in tumor cells that are critical for oncogenic function of TGF-b1. The study used breast cancer MDA-MB-231 cells in orthotopic and subcutaneous xenograft models. We show that disruption of autocrine TGF-b1 signaling in tumor cells by dominant-negative TGF-b type I receptor ALK 5-K 232R (DN-ALK5) reduces metastasis and, importantly, the micro-vascular density in both xenograft models. In contrast, active ALK5 enhances tumor angiogenesis and reduces tumor cell apoptosis. Our study identified matrix metalloproteinase MMP-9/gelatinase-B as a critical TGF-b1 target in tumor angiogenesis. Suppression of MMP-9 by RNA interference (RNAi) in tumor cells delayed tumor growth and reduced tumor neovasculature and metastasis. The analysis of the signaling pathways revealed that two kinase cascades including MEK contribute to TGF-b 1mediated up-regulation of MMP-9. Adenoviral transduction of dominant-negative MEK 1 blocked and constitutively 
active MEK 1 enhanced expression of MMP-9. In contrast, inhibition of p38 MAPK by DN-p38 alpha or RNAi-p38 alpha or JNK by kinase inhibitors had no effect. Together these findings suggested that the ALK 5-MEK-MMP-9 pathway enhances recruitment of vascular cells (endothelial cells, pericytes, myofibroblasts) from stromal compartments. To test whether stromal cells can rescue the defect in tumor angiogenesis of DNALK5 xenografts, tumor cells were placed subcutaneously alone or in combination with non-tumorigenic stromal cells. The analysis showed that stromal cells restored tumor growth and tumor angiogenesis in the DN-ALK 5 xenografts to the control levels. MRI studies of tumor xenografts confirmed the increase in blood flow in tumors containing stromal cells. Collectively, these findings demonstrate that the TGF-b 1-ALK 5MEK-MMP-9 pathway in carcinoma cells promotes tumor growth and metastasis by enhancing tumor angiogenesis via recruitment of vasculature-forming cells from stromal compartments. Additionally, we will present the progress on novel approaches for suppression of pro-oncogenic TGF-b function in tumor angiogenesis and metastasis.

\section{PD 4-5}

\section{Anti-metastatic peptide "A 5 G 27" derived from laminin $\alpha \mathbf{5}$ G-domain inhibits tumor angiogenesis}

Suguru Hibino, Masahiko Shibuya

Respiratory Division of Internal Medicine, Tokyo Metropolitan Cancer and Infection Center Komagome Hospital, Tokyo, Japan

Laminins are biologically active for cell adhesion, migration, neurite out growth, angiogenesis, tumor growth and metastasis. They are triple-helical molecules that are composed of one $\alpha$,one $\beta$, and one $\gamma$ chain. They have 5 different $\alpha, 3$ different $\beta$, and 3 different $\gamma$ chains and form a family that has at least 15 isoforms. The laminin $\alpha 5$ chain is a component of laminin-10, -11 and15. In this study, we have screened 113 overlapping synthetic peptides from the laminin $\alpha 5$ G-domain for cell attachment activity with B16-F 10 cells. Eleven attachment-active peptides were identified. In vivo experimental B16-F10pulmonary metastasis and primary tumor growth assays found that 4 of the 11 peptides inhibited tumor metastasis and growth and increased apoptosis. These four peptides (A5G27, 73, $81,101)$ also blocked tumor cell migration, invasion, and angiogenesis. We sought to identify the B16-F10 cell surface receptors for each of the four active peptides using peptide affinity chromatography. Only one peptide recognized a cell surface protein. Peptide A 5 G 27 (RLVSYNGIIFFLK, residues 2892-2904) bound a diffuse $\sim 120-180 \mathrm{kDa}$ band that eluted with $2 \mathrm{M} \mathrm{NaCl}$. Glycosidase digestion of the $2 \mathrm{M}$ eluate yielded protein bands of approximately 90 and $60 \mathrm{kDa}$ which reacted in Western blot analysis with antibodies to CD44. Immunoprecipitation of the A5G 27-bound membrane proteins with various cell surface proteoglycan antibodies confirmed CD44 as the surface receptor for A5G27. Finally, attachment assays to A5G 27 in the presence of soluble glycosaminoglycans (GAGs) identified the GAGs of CD 44 as the binding sites for A 5 G 27. Our results suggest that $\mathrm{A} 5 \mathrm{G} 27$ binds to the $\mathrm{CD} 44$ receptor of B16-F10 cells, and thus inhibits tumor cell migration, invasion, and angiogenesis. We are analyzing how A5G27 inhibits tumor angiogenesis.

\section{PD 4-6}

\section{SSeCKS metastasis-suppressing activity correlates with angiogenesis inhibition}

Irwin H. Gelman, Bing Su, Qiao Zeng, Yahao Bu

\section{Roswell Park Cancer Institute}

SSeCKS, a Src-suppressed protein kinase C substrate with metastasis suppressor activity, is the rodent orthologue of human Gravin/AKAP12, a scaffolding protein for PKA and PKC. We demonstrate here that the tetracycline-regulated re-expression of SSeCKS in MatLyLu (MLL) prostate cancer cells suppressed formation of macroscopic lung metastases in both spontaneous and experimental models of in vivo metastasis, while having minimal inhibitory effects on the growth of primary-site subcutaneous tumors. SSeCKS decreased angiogenesis in vitro and in vivo by suppressing VEGF expression in MLL tumor cells as well as in stromal cells. The forced re-expression of VEGF165 and VEGF121 isoforms was sufficient to reverse aspects of SSeCKS metastasis-suppressor activity in both the experimental and spontaneous models. SSeCKS re-expression in MLL cells resulted in the downregulation of pro-angiogenic genes such as osteopontin, tenascin $\mathrm{C}, \mathrm{KGF}$, angiopoietin, HIF-1 a and PDGFRb, and the upregulation of anti-angiogenic genes such as vasostatin and collagen 18 a, a precursor of endostatin. These results suggest that SSeCKS suppresses formation of metastatic lesions by inhibiting VEGF expression and by inducing soluble anti-angiogenic factors. 


\section{PD 4-7}

\section{Amelioration of hepatocarcinogenesis and tumor growth by combination of a clinically used angiotensin-converting enzyme inhibitor and vitamin $K$ via angiogenesis suppression}

Hitoshi Yoshiji ${ }^{1}$, Shigeki Kuriyama ${ }^{2}$, Yasuhide Ikenaka $^{1}$, Ryuichi Noguchi ${ }^{1}$, Mitsuteru Kitade ${ }^{1}$, Kosuke Kaji ${ }^{1}$, Junichi Yoshii ${ }^{1}$, Koji Yanase $^{1}$, Kiyoshi Asada $^{1}$, Masaharu Yamazaki ${ }^{1}$, Hiroshi Fukui ${ }^{1}$

${ }^{1}$ Third Department of Internal Medicine, Nara Medical University, Nara, Japan, ${ }^{2}$ Third Department of Internal Medicine, Medical School of Kagawa University, Japan

Recent studies have revealed that angiogenesis plays a pivotal role in carcinogenesis and tumor growth. The aim of our current study was to examine the combination effect of the clinically used vitamin $\mathrm{K} 2$ (VK) and angiotensin-converting enzyme inhibitor (ACE-I) on hepatocarcinogenesis induced by diethyl-nitrosamine, and orthotopic hepatocellular carcinoma (HCC) growth, especially in conjunction with angiogenesis. When used individually, both VK and ACE-I at clinically comparable low doses exerted significant inhibitory effects on the tumor development in the liver. The combination treatment of VK and ACE-I showed a more potent suppressive effect against hepatocarcinogenesis. The neovascularization increased during hepatocarcinogenesis, and VK and ACE-I significantly attenuated angiogenesis in the tumor. The hepatic expression of the vascular endothelial growth factor (VEGF) was also attenuated. In orthotopic HCC transplantation, VK and ACE-I also showed marked suppressive effects against HCC development similar to those against hepatocarcinogenesis. In both experiments, the suppressive effects of VK and ACE-I against angiogenesis were similar in magnitude to their inhibitory effects against hepatocarcinogenesis and orthotopic HCC development. In the orthotopic model, VK and ACE-I treatment resulted in a marked increase of apoptosis in the tumor, whereas tumor cell proliferation itself was not altered. Our in vitro study demonstrated that VK and PE inhibited the endothelial cell (EC) tubular formation, and that VK also suppressed the EC proliferation in a dose-dependent manner. The combination of VK and PE exerted a chemopreventive effect against liver carcinogenesis and tumor growth via suppression of angiogenesis. Since both agents are widely used in the clinical practice, this combination may represent a potential new therapeutic strategy for $\mathrm{HCC}$ in the future.

\section{PD 4-8}

\section{Protein C inhibitor suppresses tumor cell growth and metastasis by inhibiting angiogenesis}

Tomoaki Yoshikawa ${ }^{1}$, Kunihiro Asanuma ${ }^{1}$, Tatsuya Hayashi $^{2}$, Hiroki Wakabayashi ${ }^{1}$, Tatsuya Kurata ${ }^{1}$, Koji Suzuki $^{2}$, Atsumasa Uchida ${ }^{1}$

${ }^{1}$ Dept. of Musculoskeletal Surg., Mie Univ. Postgraduate School of Medicine, Japan, ${ }^{2}$ Department of Molecular Pathobiology, Mie University Graduate School of Medicine, Japan

Protein $\mathrm{C}$ inhibitor (PCI), a member of serine protease inhibitor (serpin), was initially identified in human plasma as an inhibitor of activated protein $\mathrm{C}$, the effector enzyme of the anticoagulant protein $\mathrm{C}$ pathway. PCI can also inhibit other proteases of the blood coagulation and fibrinolysis system including thrombin, thrombin-thrombomodulin complex, factor $\mathrm{Xa}$, factor XIa, plasma kallikrein, tissue plasminogen activator and urinary plasminogen activator (uPA). Like other serpins, PCI has a reactive site amino acid, which is important for inhibition of target proteases. Recently, we have demonstrated that PCI expression is significantly decreased in renal cell carcinoma tissues as compared to non-tumoral renal tissues. We have also shown that PCI inhibits the in vitro invasiveness, and the in vivo growth and metastasis of the breast cancer cell line, MDA-231 cells. It is well known that angiogenesis is closely related to growth and metastasis of cancer cells. However, the effect of PCI on angiogenesis is unknown. In the present study, we evaluated the inhibitory effect of PCI on angiogenesis using in vitro tube formation assay, and in vivo rat corneal and Matrigel implant assays. Tube formation induced by fetal bovine serum in human umbilical vein endothelial cells in the presence of PCI was significantly decreased as compared to that induced in the absence of PCI. The number of neovessels toward vascular endothelial growth factor (VEGF) containing pellet implanted into the rat cornea was significantly decreased by PCI as compared to control. Furthermore, hemoglobin content in subcutaneously implanted Matrigel containing PCIexpressing MDA-231 cells was significantly decreased as compared with that containing Mock-expressing MDA-231 cells. Overall, these results suggest that PCI is a strong inhibitor of angiogenesis. uPA degrades plasminogen to plasmin, and this directly degrades extracellular matrix or indirectly via matrix metalloproteinases, which play important roles in angiogenesis and growth of malignant tumors. The current results 
suggest that PCI suppresses growth and metastasis of cancer cells by inhibiting angiogenesis. Although PCI inhibition of uPA may explain these anti-tumoral effects, it is not unclear whether they require the protease inhibitory activity of PCI. We are presently investigating the role of PCI reactive site in its antiangiogenic activity.

\section{PD 4-9}

\section{A putative metastasis suppressor gene, Cap43/ NDRG1 regulates angiogenesis and invasion in pancreatic cancer}

Yuichiro Maruyama ${ }^{1,2,4}$, Mayumi Ono ${ }^{3,4}$, Fumihito Hosoi $^{4}$, Yuji Basaki ${ }^{4}$, Shinji Oie ${ }^{4}$, Masayoshi Kage, Hisafumi Kinoshita $^{1}$, Michihiko Kuwano ${ }^{2,4}$

${ }^{1}$ Department of Surgery, Kurume University School of Medicine, Kurume, Japan, ${ }^{2}$ Research Center for Innovative Cancer Therapy, Kurume University School of Medicine, Kurume, Japan, ${ }^{3}$ Department of Medical Biochemistry, Graduate School of Medical Sciences, Kyushu University, Fukuoka, Japan, ${ }^{4}$ Station-II for Collaborative Research, Kurume University School of Medicine, Kurume, Japan, ${ }^{5}$ Department of Pathology, Kurume University School of Medicine, Kurume, Japan

Background and purpose Cap43 has been identified as a nichel and calcium induced gene and also known as Nmyc downstream-regulated gene 1 (NDRG1), Drg-1 and rit42. It is also reported that overexpression of Cap43 suppresses metastasis of some malignancies, but its precise role remains unclear. In this study, we investigated how Cap43 expression could affect any role in tumor growth and angiogenesis by pancreatic ductal adenocarcinoma. Moreover, we also asked if Cap43 could be associated with disease progression or the malignant properties of pancreatic ductal adenocarcinoma. Results (A) Stable Cap43c DNA transfectants of pancreatic cancer cells with Cap43 overexpression showed similar growth rates as their control counterparts in vitro. By contrast, Cap43 overexpression demonstrated a marked decrease in tumor growth rates in vivo. (B) Moreover, a marked reduction in tumorinduced angiogenesis was observed. Gelatinolytic activity by matrix metalloproteinase (MMP)-9 as well as invasive ability in matrigel invasion assay was markedly decreased in pancreatic cancer cell lines with high Cap43 expression. (C) Cellular expression of MMP-9 and two major angiogenic factors, VEGF and IL-8, was also significantly decreased in cell lines with Cap43 overexpression as compared with their parental counterparts. (D) Immunohistochemical analysis demonstrated a significant association between Cap 43 expression and tumor microvascular density $(P=0.0001)$ as well as depth of invasion $(P=0.0003)$, histopathological grading $(P=0.0244)$, and overall survival rates in pancreatic cancer patients $(P=0.0062)(n=65)$. Discussion Cap43 thus could play a key role in switchon or-off of angiogenesis in tumor stroma in pancreatic ductal adenocarcinoma. A putative metastasis suppressor function of Cap43/NDRG 1 might be due to such modulation of angiogenesis in tumor stroma. Cap43 could be a novel target for angiogenesis and malignant characteristics of pancreatic cancers.

\section{PD 4-10}

\section{Growth pattern related differences of angiogenesis in hepatic colorectal adeno carcinoma metastases; impact on survival}

J. Lance Burn ${ }^{1}$, Gert G. Van den Eynden², Mark T. Swinscoe $^{1}$, Iain C. Cameron ${ }^{1}$, Ali W. Majeed ${ }^{1}$, Nigel C. Bird $^{1}$, Peter B. Vermeulen ${ }^{2}$

${ }^{1}$ Liver Research Group, University of Sheffield, Royal Hallamshire Hospital, Sheffield, S 102 JF, ${ }^{2}$ Translational Cancer Research Group (AZ Sint-Augustinus and Antwerp University), Wilrijk, Belgium

Vessel co-option, vasculogenesis and angiogenesis may all contribute to the formation of the vasculature of a hepatic colorectal metastasis. Previous work has shown that hepatic colorectal metastases grow in one of three distinct patterns or a mixture of patterns [1]. The desmoplastic pattern involves a stromal reaction around the tumour. The pushing pattern results in the compression of hepatocytes around the tumour edge and a destruction of the local hepatic vasculature, potentially leading to a hypoxic response triggering angiogenesis. The replacement pattern maintains the reticulin structure of the surrounding liver within the tumour periphery, and is associated with cooption of the sinusoidal vessels [1]. Reticulin patterns were visualised to facilitate growth pattern analysis, using a Gordon Sweet's silver stain. Double immunostaining was performed. Endothelial cells were identified with an antibody directed against CD34. Cells not in G0 were identified with an antibody directed against Ki67. Endothelial cell proliferation (ECP) rates were assessed as previously described [1]. In a series of 194 cases there were 68 desmoplastic, 28 pushing, 55 replacement and 33 mixed patterns of growth. The mean ECP $( \pm \mathrm{sem})$ from two independent observers for each pattern was $1.73( \pm 0.24)$, $3.55( \pm 0.53), 1.61( \pm 0.34)$ and $1.65( \pm 0.45)$ respectively for desmoplastic, pushing, replacement and mixed growth patterns. The median survival by a Kaplan-Meier estimation 
was $44.25,21.55,44.94$ and 26.61 months for patients with the desmoplastic, pushing, replacement and mixed growth patterns respectively. Tumours with a pushing growth pattern have higher rates of ECP compared to the other patterns of growth ( $P=0.012$ Kruskall Wallis). This type of tumour seems to be associated with reduced survival.

\section{Reference}

1. Vermeulen et al (2001) J Pathol 195:336

\section{PD 4-11}

\section{VEGF-C secreted by pancreatic cancer cell line promotes lymphatic endothelial cell migration in an in vitro model of tumor lymphangiogenesis}

Nobuo Ochi, Yoichi Matsuo, Hirozumi Sawai, Hitoshi Funahashi, Yuji Okada, Tadao Manabe

Department of Gastroenterological Surgery, Nagoya City University Graduate School of Medical Sciences, Nagoya, Japan

Objective Pancreatic cancer has the poorest prognosis of all gastrointestinal carcinomas. Prognostic factors for pancreatic carcinoma include perineural invasion, liver metastasis, and lymph node metastasis. Recently, there has been an increase in studies on mechanisms of tumorassociated lymphangiogenesis. Moreover, immunohistochemical expression of vascular endothelial growth factor (VEGF)-C in various cancers has been shown to correlate with the rate of metastasis to lymph nodes. To investigate mechanisms underlying lymphatic node metastasis in pancreatic cancer, we examined roles of VEGF-C on an in vitro model of tumor lymphangiogenesis. Methods We measured VEGF-C secretion level by pancreatic cancer cell lines using real-time RT-PCR and enzyme-linked immunosorbent assay (ELISA). Nextly, we examined effects of recombinant human VEGF-C (rVEGF-C) on lymphatic endothelial cell (LEC) proliferation using the Premix WST-1 Cell Assay System. Then, we examined effects of rVEGF-C and different pancreatic cancer cell lines derived VEGF-C on LEC migration by double chamber methods. Furthermore, we examined capillary formation by LECs using an LEC/fibroblast co-culture system on collagen gels to reflect interactions between tumor cells and the tumor microenvironment. Results Levels of VEGF-C were significantly elevated in supernatant for MIA $\mathrm{PaCa}-2$ cells $(2,270 \pm 103 \mathrm{pg} / \mathrm{ml})$, but were not detectable in supernatant for BxPC-3 cells. So, we identified VEGF-C high-secretion (MIA PaCa-2) and low-secretion cell lines (BxPC-3). The trend of enhancement of LEC proliferation by rVEGF-C was not statistically significant. Numbers of migrating cells were increased by rVEGF-C treatment in a dosedependent manner. MIA PaCa-2 cell culture supernatant caused greater LEC migration than BxPC-3 supernatant (1.61-fold). VEGFC effects were significantly inhibited by rVEGF receptor 3 (R 3)/Fc Chimera. In LEC/fibroblast co-culture on collagen gel, LEC capillary formation was significantly enhanced by coculture with MIA PaCa-2 cells compared with BxPC3 cells (1.84-fold). Enhanced capillary formation with MIA PaCa-2 cells was inhibited by rVEGF R3/Fc Chimera. Conclusions We confirmed that VEGF-C secreted by pancreatic cancer promoted LEC migration ability. Our results quantitatively indicate that LECs that had migration ability enhanced by tumor-induced VEGF-C were inclined to promote capillary formation in the tumor microenvironment in vitro. VEGF-C secreted by pancreatic cancer cells plays an important role in LEC migration occurring as part of tumor lymphangiogenesis. Because the process of tumor cell-dependent lymphangiogenesis was inhibited in the current work by blockade of VEGF-C signaling, rVEGF R3/Fc Chimera may have a role in controlling lymphatic node metastasis.

\section{PD 4-12}

Tumour-induced activation of lymphatic endothelial cells via VEGF receptor-2 is critical for prostate cancer lymphatic metastasis

Elizabeth D. Williams ${ }^{1,2}$, Yiping Zeng ${ }^{1,2}$, Kenneth Opeskin $^{3,4}$, Jeremy Goad ${ }^{5}$

${ }^{1}$ Bernard O'Brien Institute of Microsurgery, Melbourne, Australia, ${ }^{2}$ Department of Surgery (St. Vincent's Hospital) University of Melbourne, Melbourne, Australia, ${ }^{3}$ Department of Pathology (St. Vincent's Hospital) University of Melbourne, Melbourne, Australia, ${ }^{4}$ Department of Anatomical Pathology, St Vincent's Hospital) Melbourne, Australia, ${ }^{5}$ Department of Urology, St Vincent's Hospital, Melbourne, Australia

Prostate cancer disseminates initially and primarily to regional lymph nodes. However, the nature of interactions between tumour cells and lymphatic endothelial cells is poorly understood. We have isolated prostatic lymphatic endothelial cells and developed a series of twoand three-dimensional in vitro co-culture systems and in vivo orthotopic prostate cancer models to investigate 
the interactions of prostate cancer cells with prostate lymphatic endothelial cells. In vivo, PC-3 and LNCaP, but not DU145, promoted lymphatic vessel formation in the tumour periphery, showed invasion of prostatic lymphatic vessels and formed lymph node metastases. In vitro, highly lymph node metastatic prostate cancer cell lines (PC-3 and LNCaP), and their conditioned medium, enhanced prostate LEC tube formation and migration, whereas poorly lymph node metastatic prostate cancer cells (DU145) or normal prostate epithelial cells (RWPE-1), or their conditioned medium, had no effect. Furthermore, recombinant vascular endothelial growth factor (VEGF)-A and VEGF-C, but not VEGFC156S, potently promoted prostate LEC tube formation, migration and proliferation in vitro. Blockade of VEGF receptor-2 significantly reduced tumour-induced activation of lymphatic endothelial cells. These results demonstrate that tumour-induced activation of host lymphatic endothelial cells via VEGF receptor-2 is critical for prostate cancer lymph node metastasis. Thus, antiangiogenic agents targeting VEGF receptor-2 are likely to have beneficial clinical effects not only by inhibiting tumour blood vasculature formation and tumour growth, but also through antagonizing the interactions of tumour cells with lymphatic vessels.

\section{PD 4-12}

\section{Leptin-mediated neovascularization, which coordinates with VEGF, plays a pivotal role in hepatocarcinogenesis in NASH rats}

Mitsuteru Kitade ${ }^{1}$, Hitoshi Yoshiji ${ }^{1}$, Shigeki Kuriyama $^{2}$, Yasuhide Ikenaka ${ }^{1}$, Ryuichi Noguchi ${ }^{1}$, Kosuke Kaji ${ }^{1}$, Junichi Yoshii ${ }^{1}$, Koji Yanase ${ }^{1}$, Kiyoshi Asada $^{1}$, Masaharu Yamazaki ${ }^{1}$, Hiroshi Fukui ${ }^{1}$

${ }^{1}$ Third Department of Internal Medicine, Nara Medical University, Nara, Japan, ${ }^{2}$ Third Department of Internal Medicine, University of Kagawa, Kagawa, Japan

Non-alcoholic steatohepatitis (NASH), a progressive metabolic liver disease, is one of the major consequences of the current obesity epidemic. Whereas simple steatosis seems to be a benign and non-progressive condition, NASH is recognized as a potentially progressive disease that can cause hepatocellular carcinoma (HCC). The pathogenesis of NASH is a multi-factorial process, but the exact mechanism of the disease progression is still not fully understood. The aim of the current study was to elucidate the role of angiogenesis in hepatocarcinogenesis in the NASH rats. Zucker (Z) rats, which naturally develop leptin receptor mutations, and their lean littermate (L) rats were fed a choline- deficient, amino acid-defined (CDAA) diet, which induces histological changes similar to those of the human NASH; namely, hepatic steatosis, steatohepatitis, liver fibrosis, and finally HCC. With CDAA, both of Z- and L-rats showed marked steatohepatitis and elevation of the oxidative stress markers, such as 8-hydroxydeoxyguanosine (8-OHdG). In sharp contrast, the development of glutathione-S-transferase placental form (GST-P)-positive pre-neoplastic lesions, which occurs with marked liver fibrosis development, could be only observed in the L-rats but not in the Z-rats throughout 16 week CDAA treatment. The hepatic neovascularization was also significantly increased only in the L-rats. The CD 31immunopositive neo-vessels were mainly localized either along the fibrotic septa or in the GST-P-positive lesions. The hepatic expression of the vascular endothelial growth factor (VEGF) also increased only in the L-rats almost in parallel with GST$\mathrm{P}$ development. HCC and adenoma could be found only in the L-rats, whereas neither HCC nor fibrosis developed in the Z-rats throughout 80 -week CDAA treatment. Our in vitro study revealed that leptin exerted a pro-angiogenic activity only in the presence of VEGF. In conclusion, these results suggested that the leptinmediated neovascularization, which depends on the endogenous VEGF, plays a pivotal role in the hepatocarcinogenesis in NASH.

\section{PD 5-1}

\section{Chemokine receptor CXCR3 promotes colon cancer metastasis to lymph nodes}

Makoto Mark Taketo, Kenji Kawada, Hisahiro

Hosogi, Masahiro Sonoshita

Kyoto University Graduate School of Medicine (Pharmacology), Japan

Chemokines and their receptors are essential for leukocyte trafficking, and also implicated in cancer metastasis to specific organs. We have recently demonstrated that CXCR3 plays a critical role in metastasis of mouse melanoma cells to lymph nodes [1]. Here we show that some human colon cancer cell lines express CXCR3 constitutively. We constructed cells that expressed CXCR3 cDNA (DLD-1-CXCR3), and compared with non-expressing controls by rectal transplantation in nude mice. In 6 weeks, 59\% of mice inoculated with DLD1CXCR3 showed macroscopic metastasis in para-aortic lymph nodes, whereas only $14 \%$ of those with the control ( $P$ less than 0.05). In 4 weeks, DLD-1-CXCR3 expanded more rapidly than the control, although at 2 weeks, both cell lines disseminated to lymph nodes at similar 
frequencies. In contrast, metastasis to the liver or lung was rare, and unaffected by CXCR 3 expression. In clinical colon cancer samples, we found expression of CXCR3 in $34 \%$ cases, most of which had lymph node metastasis. Importantly, patients with CXCR3-positive cancer showed significantly poorer prognosis. In addition to CXCL9, CXCL10 and CXCL 11, we found that CCR7 ligand CCL21 also activates CXCR 3. These results indicate that activation of CXCR3 with its ligands stimulates colon cancer metastasis preferentially to the draining lymph nodes.

\section{Reference}

1. Kawada K et al (2004) Pivotal role of CXCR3 in melanoma cell metastasis to lymph nodes. Cancer Res 64:4010-4017

\section{PD 5-2}

\section{Lipid profiles at the tumour: liver margins in colorectal liver metastasis by imaging MALDI-MS}

Nigel C. Bird ${ }^{1}$, Sally J. Atkinson ${ }^{2}$, Malcolm R. Clench ${ }^{2}$, David Mangnall $^{1}$, Ali W. Majeed ${ }^{1}$

${ }^{1}$ Liver Research Group, University of Sheffield, Sheffield, UK, ${ }^{2}$ Biomedical Research Centre, Sheffield Hallam University, Sheffield, UK

Introduction Approximately $50 \%$ of patients with colorectal cancer will develop liver metastases within 2 years of the initial diagnosis. These patients represent the majority of people who will die as a result of their cancer. Despite the fact that large volumes of liver parenchyma may be replaced by tumour, the characteristic regeneration that takes place following surgical resection does not occur. This suggests that alternative signalling takes place between tumour and surrounding liver, subverting the normal signalling process. The lipid components of biological membranes are known to be crucial mediators of cellular differentiation and proliferation and have a significant role in intra-cellular signalling via lipid rafts. Methods Following informed consent, tissue was obtained in the operating theatre from patients undergoing partial hepatectomy for colorectal cancer metastasis (Ethics Committee registration number $\mathrm{SS} / 00 / 342$ ). After dissection, samples were snap frozen and stored in liquid nitrogen until analysis. Thirty $\mu \mathrm{m}$ sections at the margin between tumour and normal liver were cut, using a cryostat, onto aluminium slides. These were then de-hydrated in successive ethanol washes, followed by a coating of $\alpha$-CHCA $(25 \mathrm{mg} / \mathrm{ml}$ in ethanol, containing $0.2 \%$ TFA). Imaging MALDI mass spectrometry was carried out using an
Applied Biosystem "Q Star Pulser-I" hybrid QqTof instrument. Full scan mass spectra were recorded using a $200 \mu \mathrm{m}$ raster pattern. Spectra were recorded for $2.5 \mathrm{~s}$ at a laser energy of $3.4 \mathrm{~mJ}$ and a repetition rate of $1 \mathrm{kHz}$. Images were generated using Applied Biosystems/MDS Sciex "o-MALDI 4.0" software and normalised to the $m / z$ 190 matrix ion. Results Mass spectra were obtained from the tumour, liver at the margin of the tumour and healthy liver distant to the tumour. Comparison of these spectra revealed clear differences in sphingomyelin and phosphatidylcholines between the tumour and normal healthy tissue. Principal component analysis was used to identify ions contributing to the difference between healthy tissue and tumour. These were sphingomyelin 16:0 (m/z 703.5) and phosphatidylcholines with $m / z$ 734.5, 758.5, 760.5, 782.5, 786.5, 788.5 and 810.5. MALDI-MS images of the distribution of these selected phospholipids confirmed the localisation of these phospholipids to the tumour when compared to the histology. Conclusion These results show that direct chemical topographic profiling of intact biological tissues is possible, without the need for extensive sample preparation. Differences in the distribution and content of lipids between tumour and normal tissue have been demonstrated.

\section{PD 5-3}

Colonization of the metastatic site by circulating cells in a human colorectal cancer model: a neglected step in the metastatic process

Francois-Clement Bidard $^{1,2}$, Nathalie Auger ${ }^{1,3}$, Christophe Rosty ${ }^{4}$, James P. Di Santo ${ }^{5}$, Marie-France Poupon $^{1}$

${ }^{1}$ Inserm U 612, Institut Curie, Paris, France, ${ }^{2}$ Dept of Medical Oncology, Institut Curie, Paris, France, ${ }^{3}$ Dept. of Cytogenetic, Institut Gustave Roussy, Villejuif, France, ${ }^{4}$ Dept. of Pathology, Institut Curie, Paris, France, ${ }^{5}$ Inserm U 668, Institut Pasteur, Paris, France

Purpose The establishment of a favourable microenvironment could be the key point of the metastatic process, possibly leading to a colonization of a metastatic niche by circulating cancer cells unable to metastasize by themselves. Materials and methods Metastatic HT29 and non-metastatic LoVo human colorectal xenografts were used. Colony forming assays, immunocytochemistry and FISH analyses were performed to detect LoVo cells into lung metastases. Results Nonmetastatic LoVo cells do co-localize with HT29 within metastatic sites and represent up to $80 \%$ of cells within lung metastases. This colonization occurs with a mixed 
LoVo/HT29 primary tumor, but also when primary tumor sites are distinct. LoVo cells extracted from lung metastases remain non-metastatic by themselves. This colonization was not observed with non-metastatic murine melanoma B16 cells. Conclusion Our findings point at metastasis colonization as a novelly described step occurring after the conditioning of a metastatic niche. In the absence of any metastatic switch, the secondary growth of circulating cancer cells occurs only within a favourable microenvironment. This process could be of critical importance for the growth of micrometastases into metastases and also show that the monoclonal origin of metastases could be complexified by the subsequent multiclonal colonization of the metastatic site by circulating cancer cells.

\section{PD 5-4}

\section{Inhibiting effect of metallothionein on cancer} metastasis in metallothionein I/II knock-out mice

Takayuki Kondoh ${ }^{1}$, Arayo Haga ${ }^{2}$, Masahiko Satoh ${ }^{3}$, Hisamitsu Nagase ${ }^{1}$

${ }^{1}$ Gifu Pharmaceutical University, Gifu, Japan, ${ }^{2}$ Gifu Prefectural Institute of Health and Environmental Sciences, Japan, ${ }^{3}$ Laboratory of Pharmaceutical Health Sciences School of Pharmacy Aichi Gakuin University, Japan

Metallothioneins (MTs) are low molecular-metal binding proteins, and induced by wide variety of agents or factors and present in almost all cells of organisms. Although physiological function of MTs, such as detoxification of heavy metals and storage of essential metals, are well known, there have been only our several reports on the relation between MTs and metastasis. We previously reported that MTs increased the expression of matrix metalloproteinases (MMPs) and invasiveness in tumor cell in vitro and in vivo. In this study, we have investigated the role of MTs on metastasis with experimental model using MT I/II knock-out mice. To assess effect of host-derived MTs on pulmonary metastasis, murine melanoma B16-BL6 cells were injected into the lateral tail veins of $\mathrm{C} 57 \mathrm{BL} / 6 \mathrm{~J}$ MT I/II knock-out mice and the wild type mice. After 14 days, the number of lung tumor colonies was counted. The number on MT I/ II knock-out mice was $176 \pm 81$, and that on wild type mice was $49 \pm 26$. The number on the MT I/II knockout mice was about 3.6 times to that on the wild type mice. This suggests that host-derived MTs are deeply associated with inhibiting of metastasis. To examine the role of tumor cell-derived MTs, B16-BL6 cells were pretreated with $0.5,1 \mu \mathrm{M}$ cadmium $(\mathrm{Cd})$ for $72 \mathrm{~h}$ to induce MTs in the tumor cells, and the Cd concentrations did not affect the cell proliferation. The Cd pretreated tumor cells were collected and injected into the lateral tail veins of MT I/II knock-out mice. After 14 days, the number of colonies in the lung was counted. The numbers of colonies was decreased in a $\mathrm{Cd}$ concentration-dependent manner. The number were $136 \pm 81$ for the control cells, $60 \pm 21$ for the $0.5 \mu \mathrm{M}$ Cd-exposed cell and $43 \pm 33$ for the $1 \mu \mathrm{M}$ Cd-exposed cells. The MT contents in the cells exposed to various $\mathrm{Cd}$ concentrations $(0.0 .5$ and $1 \mu \mathrm{M})$ for $72 \mathrm{~h}$ were measured by the radio-immunoassay, and were increased in a Cd-concentration-dependent manner. Although effect of $\mathrm{Cd}$ is not able to exclude completely in the experiment, this result suggests that tumor cellderived MTs are also related to inhibiting of metastasis.

\section{PD 5-5}

\section{Dissecting the extravasation process of metastatic breast cancer cells using an Electric Cell-Substrate Impedance Sensing (ECIS) assay}

Janet E. Price $^{1}$, Irene F. Newsham ${ }^{2}$

${ }^{1}$ Department of Cancer Biology, University of Texas M. D. Anderson Cancer Center, ${ }^{2}$ Department of NeuroOncology, University of Texas M.D. Anderson Cancer Center, USA

Metastasis is the principal cause of death from breast cancer. Extravasation of cancer cells from the circulation is a critical step in metastasis, and is difficult to model in vitro. We are using Electric Cell Substrate Impedance Sensing (ECIS) as a tool for rapid identification of molecular regulators of extravasation. ECIS technology is based on the measurement of changes in resistance across a $0.25 \mathrm{~mm}$ electrode to the flow of small AC currents. Without cells present on the electrode, current flows unrestrained, using culture medium as an electrolyte. With cells covering the electrode, current flow is impeded proportional to the cells adhesive properties. Perturbations of an endothelial cell monolayer on the electrode by cells passing through the monolayer are measured in real time. Extravasation of cells through an endothelial monolayer is detected by a drop in resistance values, corresponding to an increased flow of current as the endothelial cells retract. The assay was used to compare the extravasation ability of the human breast cancer cell line MDA-MB-231 and isogenic variants isolated from metastases in the brain and lung of nude mice. The brain metastasis-derived BR3 variant has greater ability to form experimental brain metastases that the other variants, and greater potential 
to extravasate through a monolayer of rat brain endothelial cells, shown by a sharp decline in ECIS resistance values. The BR3 cells release more vascular endothelial growth factor (VEGF) than MDA-MB-231 cells. VEGF has been reported to disrupt endothelial barrier function and thus facilitate extravasation of metastatic cells. Expression of VEGF was suppressed in BR3 cells using siRNA, resulting in significantly less VEGF protein in culture supernatants (control $=1345 \mathrm{pg} / \mathrm{ml}$, siVEGF $=$ $23 \mathrm{pg} / \mathrm{ml}$ ). The VEGF siRNA-treated BR3 cells showed reduced extravasation through endothelial monolayers in the ECIS assays. This result suggests that elevated VEGF expressed by the BR3 cells contributes to their enhanced extravasation ability in the ECIS assay. Conclusion The ECIS assay can provide novel information about interactions between metastatic cells and endothelial cells, and molecules involved in the process of extravasation. It is also adaptable to high throughput assays for screening agents that may prevent or reduce the extravasation of metastatic cells.

\section{PD 5-6}

\section{Immunohistochemical identification of mouse heparanase isoforms in metastatic foci in lungs}

Mayumi Sue ${ }^{1}$, Michihiko Waki ${ }^{1}$, Noriko Komatsu ${ }^{1}$, Nobuaki Higashi ${ }^{1}$, Chikashi Tokuda ${ }^{2}$, Motowo Nakajima $^{3}$, Tatsuro Irimura ${ }^{1}$

${ }^{1}$ Laboratory of Cancer Biology and Molecular Immunology, Graduate School of Pharmaceutical Sciences, The University of Tokyo, Tokyo, Japan, ${ }^{2}$ Nihon Schering K. K., ${ }^{3}$ Johnson \& Johnson K. K. New Buisiness \& Technology Transfer, Japan

Background and aim Heparanase, an endo- $\beta$-D-glucronidase that cleaves heparan sulfate in extracellular matrices, was previously reported to be involved in cellular invasion associated with cancer metastasis, angiogenesis and inflammation. However, distribution and expression levels associated with individual cells were not previously investigated. B16 melanoma cells were previously reported to express high levels of heparanase. In the present report, monoclonal antibodies (mAbs) specific for different forms of heparanase were prepared and used to identify these epitopes in lung metastatic foci formed after i.v injections of B16 melanoma cells. Methods and results mAbs specific for mouse heparanase were obtained by immunization of rats with recombinant protein (precursor form), and characterized by ELISA assays using three types of recombinant heparanase, representing precursor, mature (produced by cleavage of precursor form), and C-terminal deletion form. mAbs RIO-1 and 3 required the carboxyl terminal domain because these $\mathrm{mAbs}$ did not interact with the $\mathrm{C}$ terminal deletion form, whereas mAbs RIO-6 and 7 apparently bound all variant forms. B16F10 melanoma cells grown in vitro were more strongly reactive with mAb RIO-7 than mAb RIO-1 in the immunocytochemical experiments, suggesting that the C-terminal deletion form was predominant in these cells. Lung metastases nodules formed 2 weeks after injection of B16F 10 cells were immunohistochemically examined. The staining intensity was heterogeneous and some cells seemed to express higher levels of heparanase than others within the metastatic foci. mAb RIO-1 bound more intensely than mAb RIO-7, suggesting that the precursor or mature form is more prominent in these metastatic foci. Conclusions mAbs preferentially bound to different forms of mouse heparanase were established. By the use of the combinations of those mAbs, unique characteristics of heparanase isoforms in metastatic melanoma cells are revealed.

\section{PD 5-7}

\section{Establishment of murine model for invasion- independent metastasis and evaluation in human cancers}

Takashi Sugino, Toshimitsu Suzuki

Second Department of Pathology, Fukushima Medical University, Fukushima, Japan

Metastasis model It is generally believed that active invasion by cancer cells is essential to the metastatic process. In contrast, we found an alternative metastatic pathway, named invasion-independent pathway, that does not require invasiveness in mouse mammary tumor line, MCH66 [1]. The process involves intravasation of tumor nests surrounded by sinusoidal blood vessels, followed by intravascular tumor growth in the lung, without penetration of the vascular wall during the process. We present here the characteristics of the metastasis model and a possible molecular mechanism of this pathway. We established several subclonal lines different in metastatic potential, including a highly metastatic subclone (MCH66HM) and a non-metastatic clone (MCH66C8). Biologically, highly metastatic cells showed lower invasiveness to matrigel in vitro and higher angiogenic activity to form sinusoidal tumor vessels in vivo. Differential cDNA analysis identified several genes that were overexpressed by MCH66HM, including a serine protease inhibitor (SLPI) and a tumor suppressor gene (SEMA3B). Transfection of these genes inhibited in vitro invasiveness but promoted spontaneous lung metastasis of $\mathrm{MCH} 66 \mathrm{C} 8$ 
and MCH66. These results indicate that anti-invasive molecules such as SLPI and SEMA 3 B can promote metastasis in our animal model. Human cancers We examined whether evidence for the invasion-independent pathway of metastasis is present in human cancers. Archival specimens of 10 common types of human cancers were examined for the presence of sinusoidal vasculature enveloping tumor nests and subsequently generated endothelial-covered tumor emboli in efferent veins. A percentage of tumor emboli in all cancers was found to be enveloped by endothelial cells, but these structures were particularly prevalent in renal cell carcinomas, hepatocellular carcinomas and follicular thyroid carcinomas. A common feature of the vasculature in these tumors was the presence of dilated sinusoid-like structure surrounding tumor nests. A high mean vascular area within tumors, an indication of sinusoidal vascular development, was significantly related to the presence of endothelial-covered tumor emboli. These results suggest that an invasionindependent metastatic pathway is possible in a wide variety of human cancers. Conclusions Our model may propose a new relationship between invasion and metastasis. Further investigations in the experimental system and human cancer cases may present new therapeutic strategies for the amelioration of cancer metastasis.

\section{Reference}

1. Am J Pathol 160:1973-1980, 2002

\section{PD 5-8}

\section{Exploring cancer stem cell-like feature and potential treatment of metastatic SCCHN}

Zhuo (Georgia) Chen, Dong M. Shin, Ling Su, Xin Zhang, Younghyoun Yoon, Zhongxing Liang, Hongzheng Zhang, Hyunsuk Shim

Winship Cancer Institute, Emory University School of Medicine

To understand metastasis of squamous cell carcinoma of the head and neck (SCCHN), we previously selected highly metastatic SCCHN cell lines from a poorly metastatic parental cell line through an orthotopic xenograft mouse model [1]. High incidence of lymph node and lung metastases were achieved by the selected metastatic cells, whereas rare lymph node and no lung metastases were observed from the parental SCCHN cells. Further analysis of these metastatic cells by DNA microarray and other biological assays highlighted some features of cancer stem cell (CSC) for this highly metastatic subpopulation. Our data showed that these metastatic cells expressed low levels of epithelial cell differentiation markers Ecadherin and involucrin. However, we observed high levels of mesenchymal cell marker vimentin and epithelial stem cell marker integrin $\beta 1$, compared with their parental cells. Expression of E-cadherin can be recovered by treatment with demethylation agent $5^{\prime}$ aza-deoxycytidine. Expression of involucrin was also consequently elevated after the treatment, suggesting a partial restoration of epithelial differentiation. Furthermore, these metastatic cells showed resistance to anoikis compared to their parental cells, indicating that their self-renewal activity under a detached growth condition is essential for these cells to have survived during metastasis. Similar to recent reports on lung cancer cells lacking E-cadherin, the metastatic SCCHN cells lost dependence to epidermal growth factor receptor (EGFR)-mediated growth signal and showed resistance to EGFR-targeted therapy. Alternative cellular signaling pathways such as p38-mediated pathway were suggested to support growth of these cells. Another activated signaling pathway is CXCR4-mediated pathway. We found that CXCR4 mRNA levels in all tested metastatic subclones were significantly higher than those in the non-metastatic parental cells. Treatment with a CXCR4 antagonist suppressed the primary tumor growth and inhibited lung metastasis in the orthotopic SCCHN xenograft mouse model, suggesting that CXCR4 may be a key molecule in the metastasis of SCCHN. These studies provided potential strategies in treatment of metastasis of SCCHN. (Supported by a Distinguished Cancer Scientist Development Fund of Georgia Cancer Coalition and EmTech Excellence Award to H. Shim and NIH R21 DE 014767-01 to Z. G. Chen).

\section{Reference \\ 1. Zhang et al (2002) Cancer 95:1663-1672}

\section{PD 5-9}

\section{Detection of cancer stem cells in the liver cancer}

Haraguchi Naotsugu, Ieta Keisuke, Sakashita Hiroyuki, Tanaka Fumiaki, Mimori Koshi, Matumoto Toshifumi, Mori Masaki

Department of Surgery, Medical Institute of Bioregulation, Kyushu University, Japan

Background Recently, the presence of cancer stem cell has been reported in acute myelocytic leukemia, brain neoplasms, and mammary cancer. However, cancer 
stem cells in digestive cancers have not been identified. It is important to clarify specific markers of cancer stem cells in the digestive organs to make rapid progress in cancer treatment. Material and methods In the two hepatocellular carcinoma cell lines, HuH7and Hep 3 B, side population (SP) cells that are known as stem cell rich population were evaluated by flowcytometry stained with Hoechst33342. G0 stage cell population was also identified by flowcytometry stained with Pyronin Y. Several kinds of cell surface markers, such as CXCR4, IL-6R, CD29, CD49 f, c-Met and CD133, were used to analyze with flowcytometry. G0 population was sorted and stained with cell differentiation markers, such as albumin, cytokeratin 7, 18, and 19. The ATP assay for chemoresistance was performed to with 5-FU, Gemcitabine and Doxorubicine. The tumorigenicity was evaluated by the limiting dilution method in NOD/SCID mouse with subcutaneous injection. Results SP population was identified in both $\mathrm{HuH7}$ and hep3B, and the percentage was almost $0.7 \%$. G0 stage population was also identified and Pyronin Y unstained population overlapped to the beak of the SP population. G0 population was sorted by cell sorter and stained with cell differentiation markers; albumin and cytokeratin 18 for mature hepatocyte and cytokeratin 7 and 19 for cholangiocyte. G0 population expressed both markers of hepatocyte and cholangiocyte, whereas non-G0 population expressed only hepatocyte markers. Some of specific cell surface markers were identified with flowcytometry. G0 population shows high resistance to anti-cancer drugs such as 5-FU, Gemcitabine and Doxorubicine, compared to the non-G0 population. G0 population shows high tumorigenicity compared to non-G0 population. Conclusion The hepatic cancer cell lines certainly contained some cell populations characteristic to stem cell. In these populations, interestingly, G0 population that is known as stem/progenitor population exists and plays important role in the anticancer drug resistance and high tumorigenicity. We identified common cell surface markers specific to both G0 and SP cells and these would be useful to identify cancer stem cells in the liver cancer. We are now trying to identify cancer stem cells in clinical samples and also trying to identify cancer stem cell specific molecular targets to cure cancer completely.

\section{PD 5-10}

\section{Association between expression of chemokine receptor CXCR4 and lymph node metastasis in colorectal cancer}

Shinya Fukunaga, Kiyoshi Maeda, Eiji Noda, Tamahiro Nishihara, Touru Inoue, Nobuya Yamada,
Yoshito Yamashita, Masakazu Yashiro, Tetuji Sawada, Masaichi Oohira, Kousei Hirakawa

Department of Surgical Oncology, Osaka City University Graduate School of Medicine, Osaka, Japan

Lymph node metastasis is one of the deciding poor prognosis factors of colorectal cancer. Recent studies have suggested that interaction between the chemokine receptors in several cancer cells and their ligands may play a role to help development and metastasis. CXCR4 is one of CXC chemokine receptor, and there were some reports that CXCR4 expression was associated with nodal metastasis. In this study, we examined the correlation between expression of CXCR4 and lymph node metastasis in colorectal cancer. One hundred and sixtyone consecutive patients who underwent resection at our department were studied. Out of 161 patients, lymph node metastasis was observed in $69(43 \%)$ patients, and lymphatic involvement was present in 105 (65\%) patients. Using $4 \mu \mathrm{m}$ paraffin-embedded section, immunohistochemical study was performed. The degree of immunoreactivity for CXCR4 was considered to be positive if distinct staining of the cytoplasm was observed in at least $50 \%$ of tumor cells. CXCR 4 expression was found in 87 cases $(54.0 \%)$. Regarding the correlation between nodal metastasis and the expression of CXCR4, the incidence of nodal metastasis was significantly $(P<0.01)$ higher in patients with CXCR4 positive tumors than that in those with CXCR4 negative tumors. In addition, significant correlation was observed between lymphatic invasion and CXCR4 expression $(P<0.01)$. By multivariate analysis, CXCR4, lymphatic invasion and wall invasion were independence risk factors for lymph node metastasis. This study suggested that expression of chemokine receptor CXCR4 contributed to lymphatic involvement and lymph node metastasis in colorectal cancer.

\section{PD 5-11}

\section{Essential contribution of a chemokine, CCL3, and its receptor, CCR5 to lung metastasis}

$\mathrm{Yu} \mathrm{Wu}$, Ying-Yi Li, Naofumi Mukaida

Division of Molecular Bioregulation, Cancer Research Institute, Kanazawa University, Kanzawa, Japan

Accumulating evidence suggests that tumor-associated macrophages can promote tumor progression by producing various growth factors. However, it still remains to be investigated on the regulatory mechanisms of the trafficking of alveolar macrophages and 
their roles in lung metastasis. CCL3/macrophage inflammatory protein (MIP)-1 alpha, a chemokine with a potent chemotactic activity for macrophages, and its specific receptor, CCR5, have been investigated in lung metastasis induced by an intravenous injection of a mouse renal carcinoma cell line, Renca. Multiple macroscopic lung metastasis foci appeared in lungs of wildtype mice at 21 days after an intravenous injection of Renca cells. Concomitantly, CCL3 mRNA expression was enhanced in lungs and CCL3 protein was immunohistochemically detected in tumor cells. Immunohistochemical analysis demonstrated that CCR5-positive cells accumulated mainly in the periphery of metastasis foci. Interestingly, the same treatment caused remarkably lower numbers of metastasis foci and lungs weight in either CCL3- or CCR5-deficient mice, than wild-type mice $(P<0.01)$. Moreover, the numbers of $\mathrm{F} 4 / 80$ positive cells inside metastasis foci were reduced in CCL3- or CCR5-deificient mice, compared with wildtype mice, along with intratumoral granulocyte and $\mathrm{T}$ lymphocyte numbers. Furthermore, microvessel densities in metastasis foci were reduced in CCL3- or CCR5deficient mice. The intrapulmonary mRNA expression of MMP-2 and MMP-9 was increased in wild-type mice, as metastasis foci developed and the increments were attenuated in CCL3- or CCR5-deficient mice. These observations suggest that the CCL3-CCR5 axis can regulate the MMP expression by regulating intratumoral leukocyte trafficking and eventually neovascularization at metastasis foci.

\section{PD 5-12}

\section{Linoleic acid-induced growth suppression induces quiescent cancer cell nests in nude mice}

Takamitsu Sasaki, Hitoshi Ohmori, Kiyomu Fujii, Hiroki Kuniyasu

Department of Molecular Pathology, Nara Medical University, Kashihara, Japan

Linoleic acid (LA), one of essential fatty acids, possesses opposite effects on tumor cells. One of its effects is a pro-tumoral effect as a precursor of prostaglandin E2 via metabolism by cyclooxygenases-2, which plays important roles in cancer development. Another is an anti-tumoral effect as a precursor of peroxisome proliferator-activated receptor (PPAR) $\gamma$.LA metabolites by 15-lipoxygenase-1 have biological effects as PPAR $\gamma$ ligands, which have anti-carcinogenic and anti-metastatic properties. In this study, we examined effects of LA on tumor cancer cells and on tumorigenicity of LA-treated quiescent cells to elucidate factors affect tumor formation or dormancy. In 4 cancer cell lines, cell growth was significantly inhibited by LA treatment at concentration of 200 and $400 \mu \mathrm{g} / \mathrm{ml}$. Continuous treatment with LA $(100 \mu \mathrm{g} / \mathrm{ml})$ provided growth arrest from 4 to 6 weeks after the treatment. By repetitive inoculation of LA $(100 \mu \mathrm{g} / \mathrm{ml})$, regrowing activity of these cells was gradually reduced. In comparison with the cells treated with $100 \mu \mathrm{g} / \mathrm{ml} \mathrm{LA}$, cells treated with $50 \mu \mathrm{g} / \mathrm{ml}$ LA provided quiescence status with longer treatment periods. In contrast, cells treated with $200 \mu \mathrm{g} / \mathrm{ml} \mathrm{LA}$ showed marked apoptosis and were disappeared by 4 weeks- or 5 weeks-treatment in MKN28 and Colo320 cells, respectively. MKN28 and Colo320 cells treated with or without LA $(100 \mu \mathrm{g} / \mathrm{ml})$ for 5 weeks to induce quiescence were inoculated subcutaneously into the back of nude mice. LA-pretreated MKN28 and Colo320 cells showed more numerous tumors $(9 / 10$, and $10 / 10$, respectively) than non-treated cells $(2 / 10$, and $3 / 10$, respectively) $(P<0.01)$ in nude mice. In contrast, LApretreated MKN 28 and Colo320 cells showed slower tumor growth than non-treated cells $(P<0.01)$. LApretreated MKN28 and Colo320 cells with LA administration after inoculation did not form macroscopical tumors. Histological examination revealed small cancer cell aggregation, which showed no proliferative activity. In LA-treated MKN28 and Colo320 cells, protein production of Bcl-2 was increased, whereas Bak, EGFR, and VEGF levels were decreased. These findings suggest that LA might induce quiescence and subsequent dormancy in inoculated cancer cells. Our present data suggest the mechanism of tumor dormancy. Further examination to reveal biological and molecular knowledge of mechanisms of dormancy could provide great benefits for cancer patients.

\section{PD 6-1}

\section{The impact of stromal matrix metalloproteinase-7 on osteolytic bone metastases}

\section{Conor C. Lynch, Lynn M. Matrisian}

Department of Cancer Biology, Vanderbilt University, Nashville, TN, USA

Introduction Breast cancer frequently metastasizes to bone. In the bone environment the breast cancer cells typically induce extensive osteolysis by communicating with bone stromal cells such as osteoclasts. The matrix metalloproteinases (MMPs) are a family of enzymes that collectively can degrade the entire extracellular matrix but more recently have been shown to facilitate cell-cell communication by virtue of their ability to cleave multiple growth factors and cytokines. Using a 
model of prostate tumor induced osteolytic and osteoblastic change we observed that MMP-7 expression is markedly enhanced at the tumor bone interface in comparison to the tumor area alone. Interestingly, immunohistochemical staining for MMP-7 in human and rodent samples of breast to bone metastasis revealed that osteoclasts are the major source of MMP-7. In the current study we assessed whether the ablation of stromal MMP-7 will have an impact on the progression of mammary tumor cells in the bone. Methods An osteolytic luciferase tagged polyoma virus middle $\mathrm{T}$ (PyVT-Luc) mammary tumor cell line was injected into the tibia of 6 week old female immunocompromised wild type $(n=14)$ and MMP-7 $(n=9)$ deficient animals. Luciferase and microCT imaging modalities were used to follow tumor growth and osteolysis over a three week period. Mice $(n>$ or $=3)$ were harvested from each group at weekly intervals to histologically assess osteoclast recruitment using the cytochemical marker tartrate resistant acid phosphatase (TRAP) and the extent of bone destruction using histomorphometry. Results Analysis of the tumor growth rates using luciferase data demonstrated that the absence of stromal MMP-7 significantly inhibited tumor progression in comparison to the wild type controls. A significant decrease in the extent of osteolysis was also observed in the MMP-7 deficient animals in comparison to controls using microCT scanning. This decrease in bone destruction correlated with a decrease in TRAP positive osteoclasts. Immunohistochemistry identified that in the wild type mice injected with PyVT-Luc, the major source of MMP-7 was derived osteoclasts. Conclusions Our results demonstrate that osteoclast derived MMP-7 contributes to mammary tumor induced osteolysis. Based on our previous observations in prostate tumor induced osteolysis, we anticipate that a lack of MMP-7 solubilized RANKL may be responsible for the decrease in tumor induced osteolysis in the MMP-7 deficient animals. These studies add to the growing literature that supports the use of selective MMP inhibitors for the treatment of osteolytic bone metastases.

\section{PD 6-2}

\section{MMP13 promotes prostate tumor growth in the bone microenvironment by activating TGF- $\beta$}

Mitsuru Futakuchi, Shin-ya Sato, Tomoyuki Shirai

Department of Experimental Pathology and Tumor Biology, Nagoya City University Medical School, Nagoya, Japan
Bone is one of the most common sites of metastasis in human prostate cancer, and interactions between host cell (osteoblasts and osteoclasts) and metastatic cancer cells have been shown to play important roles of bone metastasis formation. We recently developed an in vivo model, which allows us to examine the molecular mechanisms underlying tumor growth in the bone microenvironment. Transplantation of rat prostate tumor (PLS-P) onto the cranial bone allowed us to observe tumor growth with osteoblastic and osteolytic changes at the tumor bone interface (TB-interface), and the histological features at TB-interface mimics that of metastatic bone lesions in human prostate cancer. Transforming growth factor (TGF)- $\beta$, stored within the bone matrix, is released locally in the microenvironment as a consequence of osteoclastic bone resorption. Therefore, we hypothesized that suppression of osteolysis would reduce TGF- $\beta$ levels, which would consequently inhibit growth of prostate cancer in the bone microenvironment. Using our animal model, we examined the effect of two bisphosphonates (Minodronate and Incadronate), which was shown to reduce the induction of osteoclast, on osteolytic and osteoblastic changes associated with the growth of rat prostate tumors. Minodronate suppressed both osteolytic and osteoblastic changes whereas Incadronate suppressed only osteolytic changes. However, neither bisphosphonate reduced cell proliferation of prostate tumors at the TB-interface in spite of strong suppression in osteolysis. ELISA data demonstrated that active TGF- $\beta$ level but not total (active + latent) TGF- $\beta$ level was correlated with tumor cell proliferation. The index of immunohistochemically positive for phosphorylated Smad 2, which reflects the degree of endogenous TGF- $\beta$ signal transduction, was correlated with tumor cell proliferation at the TB-interface. These data suggest that a protease, necessary for activation of TGF- $\beta$, may be involved in prostate tumor growth in the bone microenvironment. Microarray analysis at the TB-interface revealed that MMP 13 were up-regulated at the TBinterface while proteases that are known for activating TGF- $\beta$ were not. We found MMP13 was produced by osteoblast by immunohistochemical stainings. Further investigation revealed that administration of ONO4817, which predominantly inhibits MMP13 activity, significantly reduced tumor volume increased and osteolysis. In conclusion, these findings clearly indicate that MMP13 derived from osteoblasts, activated TGF- $\beta$ at the TB-interface, which result in promoting prostate tumor growth in the bone microenvironment. Therefore, MMP13 may be a therapeutic target for bone metastasis of prostate cancer. 


\section{PD 6-3}

2-Methoxyestradiol (2ME2) suppresses bone metastasis and osteolysis by activating TGF- $\beta$ signaling

Muzaffer Cicek ${ }^{1}$, Urszula T. Iwaniec ${ }^{2}$, Michael Goblirsch $^{3}$, Denis R. Clohishy ${ }^{3}$, Russel Turner ${ }^{2}$, Merry Jo Oursler ${ }^{1}$

${ }^{1}$ Endocrine Research Unit, Mayo Clinic College of Medicine, Rochester, MN, USA, ${ }^{2}$ College of Health and Human Sciences, Oregon State University, Corvallis, OR 97331, USA, ${ }^{3}$ Department of Orthopedic Surgery, University of Minneapolis, Minneapolis, MN 55455, USA

2-Methoxyestradiol (2ME2), a physiological metabolite of $17 \beta$-estradiol (estrogen), has recently emerged as a promising cancer therapy because of its potent growthinhibitory and pro-apoptotic effects on both endothelial and tumor cells. Using a mouse model of osteolytic tumor progression, we sought to determine 2ME2 effects on tumor growth in bone and osteolysis. We generated a 4T1 murine metastatic breast cancer cell line that stably expressed Far Red fluorescence protein (4T1/Red) to visualize tumor development. One thousand 4T1/Red cells were injected into bone marrow of the left femur and the mammary pad of 6- to 7-week-old Balb/c mice. Tumor progression and bone metastasis were monitored using a KODAK IS4000 imaging system. 2ME2 treatment began on the same day as surgery and was administered subcutaneously at 10, 25, and $50 \mathrm{mg} / \mathrm{kg}$ doses daily. Our results clearly show that 2ME2 inhibits tumor growth in both bone and soft tissue, tumor cell metastasis to bone, and osteolysis in vivo in a mouse model of metastatic breast cancer with maximum effects at $50 \mathrm{mg} / \mathrm{kg} /$ day. Bone mass was determined by micro-CT, which documented that tumor-induced osteolysis was significantly reduced in the bones from mice receiving $2 \mathrm{ME} 2$. Interestingly, in addition to osteolytic tumor growth in the injected limb, osteolytic tumors were also observed in the contra-lateral limb. To determine the origins of these metastatic tumors, we injected tumor cells into the bone marrow or the mammary pad of separate mice and found that metastasis to the non-injected limb arose from both bone and soft tissue injections. Since 2ME2 induces tumor cell apoptosis, we examined its effects on tumor cell signaling and we have found that 2 ME2 simultaneously increased MKK4 and JNK phosphorylation and induces Smad 2/3 translocation to the nucleus in tumor cells. These events are followed by increased TGF- $\beta$ Inducible Early Gene (TIEG) expression. Since studies have highlighted that TIEG induces tumor cell apoptosis, TIEG may be an important tumor suppressor gene leading us to hypothesize that $2 \mathrm{ME} 2$ stimulates apoptosis by activation of JNK and Smad2/3 nuclear translocation and increasing TIEG expression in breast cancer cells. These data suggest that overexpression of TIEG in breast cells would slow tumor development, and tumor dissemination, as well as repress tumor-induced osteolysis.

\section{PD 6-4}

\section{TGF- $\beta$ promotes prostate tumor growth in the bone microenvironment}

\section{Shin-ya Sato, Mitsuru Futakuchi, Tomoyuki Shirai}

Department of Experimental Pathology and Tumor Biology, Nagoya City University Medical School, Nagoya, Japan

Prostate cancer is the most commonly diagnosed and the second leading cause of death from cancer among men. The most common site for metastasis is bone and patients with bone metastasis are incurable because bone metastasis is resistant to conventional therapy. Prostate tumor growth in the bone microenvironment is associated with osteoblastic and osteolytic changes. Osteolysis is caused by osteoclast stimulation and bidirectional interactions between tumor cells and osteoclast lead to both osteolysis and tumor growth in the bone microenvironment. The most abundant repository for transforming growth factor (TGF)- $\beta$ is the bone matrix, and it is released locally as a consequence of bone resorption. We recently developed an in vivo model, which allows us to examine the molecular mechanisms underlying tumor growth in the bone microenvironment. Transplantation of rat prostate tumor (PLS-P) onto the cranial bone allows us to observe tumor growth with osteoblastic and osteolytic changes at the tumor bone interface (TB-interface). To test our hypothesis that TGF- $\beta$ plays an important role in promoting prostate tumor growth in the bone microenvironment, we compared the differences in the tumor growth, TGF- $\beta$ levels, and TGF- $\beta$ signal transduction between in the bone microenvironment and in the subcutaneous environment. The data showed that no significant difference in the size of the tumor on the cranial bone versus the subcutaneous lesion. Histological examination revealed that induction of osteoblasts/osteoclasts, and osteoblastic/osteolytic changes was present at the tumor bone interface (TB-interface) of the cranial tumor. Whereas, such changes were not observed at the tumor stromal interface (TS-interface) of 
the subcutaneous lesion. ELISA data showed that active TGF- $\beta 1$ levels at the TB-interface were significantly higher than that at the TS-interface. The index of phosphorylated Smad2, that reflects the degree of endogenous TGF- $\beta$ signal transduction, was also significantly higher at the TB-interface than that at the TSinterface. These results indicate that TGF- $\beta$ signaling transduction was elevated in the bone microenvironment. Cell proliferation activity of prostate tumors, which was evaluated by PCNA staining index, was higher at the TB-interface than that at the TS-interface. In conclusion, our data showed TGF- $\beta$ signaling transduction was well-correlated with the growth of prostate tumor in the bone microenvironment.

\section{PD 6-5}

\section{A classic set of osteoblast-derived inflammatory cytokines is produced in response to bone metastatic breast cancer}

Andrea M. Mastro, Karen M. Bussard, Elizabeth M. Chislock, Michelle Kinder, Carol V. Gay

\section{Penn State University, USA}

Breast cancer frequently metastasizes to bone and disrupts the balance between bone resorbing osteoclasts and bone depositing osteoblasts. During metastatic breast cancer cells invasion, osteoclasts are indirectly activated to degrade bone and osteoblasts cease to deposit new bone. Current therapies which utilize bisphosphonates to block osteoclast function are not curative. While osteolytic lesion progression is slowed, the lesions do not heal. Presently, the mechanism by which this disease occurs is unclear. We previously reported that osteoblasts no longer produce non-collagenous bone matrix proteins. The focus of this project was to determine how osteoblast-derived cytokine production was altered in response to metastatic breast cancer cells or their conditioned medium (CM). Alterations in osteoblast-derived cytokine production were examined using Raybio species-specific cytokine arrays and quantified with standard ELISAs. Preliminary in vitro experiments utilized hFOB 1.19 human fetal osteoblasts. Subsequent experiments were performed using MC3T3-E1 murine osteoblasts incubated with breast cancer cell CM. In addition, femurs from untreated or cancer bearing female athymic mice inoculated by intracardiac injection with MDA-MB-231 human metastatic breast cancer cells were assayed at various times for the presence of inflammatory cytokines. Murine femurs were harvested, minced, and incubated for $24 \mathrm{~h}$ in culture medium. Incubation of osteoblast cell lines with metastatic breast cancer $\mathrm{CM}$ led to enhanced expression of osteoblast inflammatory stress molecules KC, IL-6, MIP-2, and MCP-1. Cultures of metaphyses of long bones cleared of bone marrow from untreated mice expressed cytokines different from those in the diaphysis (predominantly KC, MIP-2, and MCP-1). Cultures of femurs from cancer bearing animals showed elevated levels of these inflammatory cytokines. These results suggest metastatic breast cancer cells induce osteoblasts to undergo a stress response and produce inflammatory cytokines. Additionally, cytokines were specifically produced by cells of the bone and not marrow stromal cells. From these results, we believe osteoblast-derived factors are as integral, if not more important, than breast cancer-derived factors in modifying the metastatic environment. Furthermore, inflammatory cytokines produced by osteoblasts may attract and activate osteoclasts, thereby supporting a favorable bone microenvironment for metastatic breast cancer cells. By identifying alterations in osteoblast-derived cytokines specific to the presence of metastatic breast cancer cells, it may be possible to block or disrupt these factors through the use of targeted drugs. Supported by Sigma $\mathrm{Xi}$, The Pennsylvania State University President Fund for Research, National Foundation for Cancer Research Center for Metastasis Research, and U. S. Army Medical Research and Materiel Command W81XWH06-1-0363.

\section{PD 6-6}

\section{Estrogen regulated metastasis-associated genes in breast cancer bone metastases}

Anait S. Levenson, Jun Wang, Yeon T. Chung, Jennifer Jarrett, Robert L. Satcher

Orthopaedic Surgery Department \& Comprehensive Cancer Center, Northwestern University, Chicago, USA

Clinical observations imply a possible role of hormones and estrogen receptor (ER)-signaling pathways in the development and progression of breast cancer bone metastases. Thus, although expression of $\mathrm{ER} \alpha$ is generally considered to be a good prognostic marker while the role of $\operatorname{ER} \beta$ is uncertain, data suggest of biologically diverse sets of ER-positive tumors associated with high frequency of developing bone metastases. Our goal is to identify "molecular signatures" of such ER-positive breast cancers, which can potentially be used as prognostic markers and therapeutic targets for prevention and treatment of bone metastases. Our objectives were to (1) establish in vitro hormone-responsive co-culture system 
consisted from $\mathrm{ER} \alpha$ - or $\beta$-positive metastatic breast cancer cells and $\mathrm{ER} \alpha$ - or $\beta$-positive osteoblasts; (2) examine transcriptional activation/modulation of ER $\alpha$ and $\mathrm{ER} \beta$ mediated gene expression by $17 \beta$-estradiol (E2) and selective ER modulators (SERMs) in co-cultured cancer/bone cells using cDNA microarrays; (3) analyze expression of selected E2-responsive molecular markers in specimens from ER-positive patients in different stages of breast cancer using tissue microarrays and correlate results with clinical outcome, i.e. bone metastases. Expression of ERs in cancer (MDA-231) and bone cells (U2OS) were validated by Western blots, and bi-compartmental co-culture conditions were optimized by examining known E2-responsive genes involved in "vicious cycle" using Northern blots. To identify additional E2-regulated metastasis-associated genes in cocultured cancer/bone cells we utilized custom-made "tumor metastasis", "ER-signaling" and "osteogenesis" microarrays. Sets of "E2-effects" genes were identified in cancer cells and osteoblasts. Among genes that were increased in MDA-ER $\alpha$ cells as a result of cancer/bone interaction and E2 exposure was osteopontin, bone-related protein, which induces migration in cancer cells and MacMarcks, which belongs to a family of PKC substrates and controls cell shape changes. On the other hand, uPA, MMPs and others that contribute to breast cancer cell metastatic potential were down-regulated by E2. In addition, E2 decreased $\mathrm{ER} \beta$-mediated transcription of MUC1, whose overexpression by epithelial cancers is associated with poor survival. Interestingly, E2 effects in co-cultured cancer cells were mediated through both $\mathrm{ER} \alpha$ and $\mathrm{ER} \beta$, whereas in bone cells gene alterations were almost exclusively ER $\beta$-mediated. E2 treatment resulted in decreases in mRNAs for CSFs, TGF $\beta$ R2, collagens, BMP3 and Twist. Further analysis of microarray data will disclose critical E2-responsive metastasis-associated genes relevant in progression of bone metastasis and suggest new strategies of hormonal therapy in advanced breast cancer bone metastatic disease. Supported by Susan G. Komen Breast Cancer Foundation award to ASL.

\section{PD 6-7}

\section{Novel chemoprevention with paclitaxel loaded hydroxyapatite-alginate particles for paralysis in metastatic spine cancer in rats}

Tetsuya Abe ${ }^{1}$, Masataka Sakane ${ }^{1}$, Toshiyuki Ikoma ${ }^{2}$, Tomohiko Yoshioka ${ }^{2}$, Junzo Tanaka ${ }^{3}$, Naoyuki Ochiai ${ }^{1}$

${ }^{1}$ Department of Orthopedic Surgery, and Graduate School of Comprehensive Human Sciences, University of Tsukuba, Ibaraki, Japan, ${ }^{2}$ Biomaterials Center,
National Institute for Materials Science, Tsukuba, Ibaraki, Japan, ${ }^{3}$ Department of Metallurgy and Ceramics Science, Tokyo Institute of Technology, Meguro, Tokyo, Japan

Introduction Bone tissues are one of the most frequent sites for breast cancer metastasis. New combined anticancer drug regimens including taxanes have improved survival in breast cancer patients; however, treatment of bone metastasis remains the most challenging task in cancer therapy because therapeutic agents hardly reach bone tissues. Many researchers have tried to develop a more effective formulation of paclitaxel. Materials and methods: We developed novel $7.4 \mathrm{wt} \%$ paclitaxel-loaded hydroxyapatite-alginate particles for this study. The CRL-1666 mammary adenocarcinoma cell line was selected and an intraosseous spinal metastatic tumor model was used to test the efficacy of local treatment. Thirty 8-week-old Fischer 344 female rats undergoing surgery and tumor implantation were randomized into 3 groups, control group $(n=8)$, systemic treatment group $(n=10)$ and local treatment group $(n=12)$. The B-B-B scale was used to evaluate hind-limb motor function. Paralysis-free survival (PFS), defined as the latency time of paralysis, was calculated using KaplanMeier product-limit methods. The log-rank test and Fischer's PLSD test following ANOVA analysis were used to define statistical significance. $(P<0.05)$. Results All 8 rats in the control group developed hind-limb paralysis in a mean of 9 days with a consistent decrease in B-B-B scale, and died in a mean of 14.75 days after surgery. Nine rats in the systemic treatment group and 11 rats in the local treatment group developed hind-limb paralysis in a mean of 10.4 days, 12.8 days respectively. The mean PFS in the local treatment group was significantly longer than that in the control group $(P<0.05)$. The mean B-B-B score in the local treatment group remained significantly higher than the control group and systemic treatment group over 2 weeks after surgery $(P=0.0028, \quad P=0.0025$ respectively). Discussion There is a direct relationship between the extent of osseous involvement and patient survival, particularly spinal cord compression. We assume that local administration using a novel drug delivery system would be an attractive option for treating bone metastasis. If these particles resulted in clinical efficacy, a novel local treatment would become feasible by minimally invasive surgery. However, the in vitro pharmacokinetics and mechanical strength of the implanted particles need further investigation. Conclusions We successfully developed novel paclitaxelloaded hydroxyapatite-alginate particles. In our study, paralysis-free survival was significantly prolonged by 
local administration of the particles with no sign of toxicity in an intraosseous spinal tumor model in rats. Our result allows the further preclinical evaluation of novel chemoprevention for patients harboring metastatic spine cancer.

\section{PD 6-8}

\section{A new in vitro model of breast cancer metastasis in bone}

Andrea M. Mastro, Laurie A. Shuman, Ravi Dhurjati, Xiaomei Lui, Carol V. Gay, Erwin A. Vogler

\section{Penn State University, USA}

Breast cancer frequently metastasizes to bone, where it activates osteoclasts to form osteolytic lesions. Although bisphosphonates inhibit osteoclasts and slow lesion progression, the lesions do not heal indicating that breast cancer also disrupts osteoblasts. We found that metastatic breast cancer cells can inhibit MC3T3E1 osteoblast differentiation, increase osteoblast apoptosis, cause the osteoblasts to change morphology, as well as produce inflammatory cytokines, such as IL-6 and MCP-1, which are known to recruit and activate osteoclasts. In vivo studies verified that the observations made using cell culture are physiologically relevant; however, they are time consuming and expensive. Further, animals are difficult to monitor and manipulate in order to investigate mechanisms. Therefore, we have developed a bioreactor to bridge the gap between conventional culture models and animal models. We plan to use the bioreactor to test the hypothesis that breast cancer metastases to bone affect osteoblast function. The bioreactor consists of two compartments. The upper serves as a medium reservoir, while the lower contains the cells. Preliminary data indicate that MC3T3E1 or hFOB1.19 osteoblasts can be cultured for more than 120 days. In contrast, conventional culture allows the cells to be cultured for approximately 30 days. In the bioreactor, osteoblasts produce alkaline phosphatase, and form a mineralized, 3-D matrix. We have cocultured GFP-MDA-MB-231 cells, a human metastatic breast cancer line, with osteoblasts in the bioreactor and observed changes in osteoblast morphology, a decrease in density, apoptosis, as well as an increase in inflammatory cytokines similar to the observations made in cell culture and in mice. Interestingly the cancer cells quickly migrated through the osteoblast multilayer. We are currently developing methods to introduce osteoclasts along with the osteoblasts in order to more accurately mimic the in vivo bone microenvironment. Thus, the bioreactor provides an in vitro approach that closely resembles in vivo conditions. It holds promise as a substitute for animals in research and drug testing. We are using it to investigate the mechanisms involved in breast cancer induced osteoblast inhibition of biosynthetic bone in order to develop therapeutic targets for treatment of lesions. Support: Huck Institute Innovation Group; The Penn State Cancer Institute; National Foundation for Cancer Research, US Army Breast Cancer Program Idea Award W81XWH-06-1-0432.

\section{PD 6-9}

\section{The application of an engineered murine bone bioreactor as a model of experimental bone metastasis}

Conor C. Lynch ${ }^{1,2}$, Jennifer Halpern ${ }^{1}$, Michelle D. Martin $^{2}$, Herbert S. Schwartz' ${ }^{1}$ Lynn M. Matrisian², Ginger E. Holt ${ }^{1}$

${ }^{1}$ Department of Orthopedics and Rehabilitation, Vanderbilt University, Nashville, TN, USA, ${ }^{2}$ Department of Cancer Biology, Vanderbilt University, Nashville, TN, USA

Introduction Using tissue engineering techniques, we have used bone morphogenetic protein-2 (BMP-2) and an osteoconductive hydroxyapatite scaffold ligated to the murine femoral artery to generate an in vivo bone bioreactor. The bioreactor facilitates the investigation of questions regarding bone formation or pathological bone conditions such as bone metastasis. In the current study we have applied the bone bioreactor as a model of mammary tumor extravasation and growth. Materials and methods The femoral artery of 6 week old FVB mice was ligated, excised and threaded through a hydroxyapatite scaffold that was pre-treated with BMP-2 $(20 \mu \mathrm{g} / \mathrm{cm} 3)(n=17)$, or with saline as a control $(n=15)$. The bioreactor was removed and histologically assessed at 6 and 12 week time points. For studies relating to the application of the bioreactor as a model of bone metastasis, FVB mice that were 6 weeks post implantation of BMP-2 $(n=7)$ or control $(n=5)$ treated coral were injected with syngeneic luciferase tagged polyoma virus middle $\mathrm{T}$ antigen (PyVT-Luc) mammary tumor cells via the femoral artery feeding the bioreactor. Results Immunohistochemical staining for Von Willebrand factor demonstrated that the extent of vascularization was similar in the control and BMP-2 treated groups. Staining for collagen type I, alkaline phosphatase and tartrate resistant acid phosphatase demonstrated that BMP-2 significantly enhanced bone formation in the bioreactor in comparison to the controls. Growth of the PyVT-Luc cells was determined by real time imaging of luciferase expression using the 
in vivo imaging system (IVIS ${ }^{\mathrm{TM}}$ ) from Xenogen. We observed that the presence of bone in the implanted bioreactors significantly enhanced the growth rate of the cells in comparison to the control group. Conclusions We have developed a unique in vivo model of bone engineering and demonstrated that it can be manipulated to address molecular questions that pertain to bone development and pathological bone conditions such as breast to bone metastasis.

\section{PD 6-10}

\section{Effect of soluble vascular endothelial growth factor receptor 1 (sVEGFR1) on growth of human prostate cancer cells in the bone of nude mice}

Hisanori Uehara, Keisuke Izumi

Department of Molecular and Environmental Pathology, The University of Tokushima Graduate School, Tokushima, Japan

Vascular endothelial growth factor (VEGF) plays an important role on progression and metastasis of prostate cancer. VEGF secreted from cancer cells, binds with highaffinity to the tyrosine kinase receptors Flt-1 (VEGFR-1) and Flk-1/KDR (VEGFR-2) expressed by endothelial cells, and exhibits angiogenic activity. We determined whether soluble VEGFR1 (sVEGFR1), which bind to VEGF and sequesters this ligand before its binding to cellular receptor, can inhibit growth of human PC-3 prostate cancer cells in the bone of nude mice. ThesVEGFR1cDNAwas obtained from human umbilical vein endothelial cells (HUVEC) by RT-PCR, and was inserted into a mammalian expression vector, pIRESneo3 (pIRESsVEGFR1). Human PC-3 prostate cancer cells were transfected with pIRESneo3 (PC3mock) or pIRESsVEGFR1 (PC3-sVEGFR1) and cultured in neomycin (G418)-containing medium, then stable transfectants were obtained. Expression of sVEGFR1 mRNA was found in PC3-sVEGFR1 cells, but not in PC3-mock cells by RTPCR. Secretion of sVEGFR1 was detected only in PC 3sVEGFR 1-cultured medium by ELISA. PC 3-sVEGFR1 cells or PC-3-mock cells were directly injected into the tibia of 8 week-old male nude mice $\left(5 \times 10^{5}\right.$ cells/mouse). Bone lesions were monitored by radiography. At week 3 , small bone lesions were observed in 2 of 5 PC-3-mock-injectedmice and 1 of 5 PC3-sVEGFR1-injected-mice. At week 5, bone lesions were observed in all PC-3-mock-injectedmice. In contrast, the number of PC3-sVEGFR1injected-mice with bone lesion was not increased, although the lesion, which was observed at week 3 , was advanced. Our results suggest that sVEGFR1 can inhibit growth of human PC-3 prostate cancer cells in theboneofnudemice.

\section{PD 7-1}

\section{Significance of podoplanin-positive lymphatic microvessel density in patients with esophageal carcinoma}

Yoshifumi Nakayama, Kentaro Matsumoto, Masaru Nagato, Yuzuru Inoue, Takefumi Katsuki, Noritaka Minagawa, Kazunori Shibao, Yosuke Tsurudome, Keiji Hirata, Tatsuhiko Sako, Naoki Nagata

Department of Surgery 1, University of Occupational and Environmental Health, Japan

Background Although the lymph node involvement are important prognostic factor for survival in patients with esophageal carcinoma, little is known about the lymphangiogenesis in the esophageal carcinoma. Podoplanin, a mutintype transmembrane glycoprotein, is specifically recognized the lymphatic endothelium and used as a lymphatic-specific marker. Therefore, we decided to use anti-human podoplanin antibody for quantify and evaluation of the lymphangiogenesis in the esophageal carcinoma. Materials and methods The lympharic endothelial cells were detected by immunohistochemistry using a mouse monoclonal antihuman podoplanin antibody. We investigated the relationship between lymphatic microvessel density (LMVD) and lymphatic vessel invasion (LVI), clinicopathological factors and prognosis in 29 patients with esophageal carcinoma. Results LMVD was significantly higher in esophageal cancer patients who had any of the following characteristics: T3-T4 $(P=0.0370)$, tumors more advanced than stage III (TNM) $(P=0.0351)$, lymphatic invasion $(P=0.0095)$ and LVI $(+)(P=0.0016)$. LVI significantly correlated with lymph node metastasis $(P=0.0003)$, TNM staging $(P=0.0182)$ and LMVD $(P=0.0388)$. The survival rate of patients with low LMVD tended to be higher than that of patients with high LMVD (5-years survival rate, $62.5 \%$ vs. $29.4 \%, P=0.0832$ ). Conclusions The evaluation of the lymphangiogenesis by podoplanin immunohistochemistry may be important to predict the lymph node metastasis or prognosis in the patients with esophageal carcinoma.

\section{PD 7-2}

\section{A novel cancer testis antigen that is frequently expressed in colorectal cancer}


Takeshi Yokoe ${ }^{1}$, Fumiaki Tanaka ${ }^{1}$, Takahiro Omachi ${ }^{2}$, Koshi Mimori ${ }^{1}$, Hiroshi Inoue ${ }^{1}$, Masato Kusunoki ${ }^{3}$, Masaki Mori ${ }^{1}$

${ }^{1}$ Department of Molecular and Surgical Oncology, Medical Institute of Bioregulation, Kyushu University, Beppu, Japan, ${ }^{2}$ Department of Surgery, Jikei University School of Medicine, Tokyo, Japan, ${ }^{3}$ Department of Gastrointestinal and Pediatric Surgery, Division of Reparative Medicine, Institute of Life Sciences, Mie University Graduate School of Medicine, Mie, Japan

Advanced and metastatic colorectal cancer is a common disease with an high mortality rate. Immunotherapy is novel therapeutic approaches to treat patients with colorectal cancer. For the development of peptidebased, cancer specific immunotherapy, the identification of new cancer-testis antigens is very important. But cancer-testis antigen expression is rarely observed in colorectal cancer. In search for genes associated with colorectal cancer by cDNA microarray, we found the candidate gene for cancer-testis antigen upregulated in colorectal cancer. The mRNA expression of the gene was evaluated in 76 clinical samples of surgically resected colorectal cancer by reverse transcription-PCR. The expression of the gene in normal tissues also evaluated by human RNA panels (clontech Laboratories). The production and localisation of the protein of this gene were investigated by immunohistochemistry. This gene was overexpressed in 66 of $77(86 \%)$ colorectal cancer patients in cancerous tissues compared with normal counterparts $(P<0.0001)$. The elevated expression of this gene was not correlated with clinicopathological significance. Expression in normal tissues was highly in testis and weakly in placenta, but no expression was observed in other tissues. Moreover, the expression of this gene was not overlapped with that of MAGE genes. Immunohistochemical staining with polyclonal antibody in colorectal cancer cells and normal mucosa indicated that this gene was only expressed in the cancer cells. These data suggest that this gene is a new cancer-testis antigen and may be useful for immunotherapy for patients with colorectal cancer. At the present time we try to identify the tumor-associated $\mathrm{T}$-cell epitopes for vaccine development.

\section{PD 7-3}

The roles of loss of heterozygosity at D8S298 in the molecular classification of patients with hepatocellular carcinoma: with special reference to survival after surgical operation

Lun-Xiu Qin, Jin-Zhong Pang, Ning Ren, Qing-Hai Ye,
Zhao-You Tang

Liver Cancer Institute and Zhongshan Hospital, Fudan University, Shanghai, China

Purpose Our previous studies have demonstrated that chromosome $8 \mathrm{p}$ deletion, particularly $8 \mathrm{p} 11-12$ and $8 \mathrm{p} 21$ 23 , correlates with metastasis of human hepatocellular carcinoma (HCC). The purpose of this study was to determine whether allelic losses on these regions including $8 \mathrm{p}$ could be used in predicting the prognosis of patients with HCC. Experimental design A total of 208 patients with TNM stage I or II of HCC who underwent curative liver resection from January 1999 to March 2000 were enrolled. Genomic DNA was extracted from microdissected HCC tissues. LOH was examined using 10 microsatellite markers located at the regions of chromosome $8 \mathrm{p}$ mentioned above, and its association with 5-year overall survival (OS) and disease-free survival (DFS) of patients was analyzed. Results The mean heterozygosity of these loci was $69.8 \%$. The LOH frequencies assessed from informative cases at the loci tested were also high, with a range from $32.6 \%$ (D8S1721) to $48.8 \%$ (D8S261), and the three most frequently altered loci were D8S261 (8p22). In univariate analyses, LOH on D8S298 (8p22) $(39.6 \%)$ was associated with a worse 5-year OS $(P=0.037)$ and DFS $(P<0.001)$ of the entire cohort of patients. And more, even in those patients with TNM stage I of HCC, LOH D8S298 were also found to be associated with decreases in both OS $(P=0.008)$ and DFS $(P=0.038)$. In a multivariate model, D8S298 LOH was one of the independent predictors for decreased DFS $(P=0.019)$ and decreased OS $(P=0.014)$. Conclusion $\mathrm{LOH}$ at D8S298 is independently associated with a worse survival in stage I and II patients with curative resection for HCC.

\section{PD 7-4}

Evaluation of sentinel node concept in gastric cancer based on lymph node micrometastasis determined by reverse transcription-polymerase chain reaction

Tsutomu Kozono, Shoji Natsugoe, Yoshikazu Uenosono, Takaaki Arigami, Shigehiro Yangaita, Katsuhiko Ehi, Hideo Arima, Yuko Mataki, Sumiya Ishigami, Takashi Aikou

Department of Surgical Oncology, Digestive Surgery, Kagoshima University, Kagoshima, Japan

Objective To determine the adequacy of sentinel node (SN) concept based on micrometastasis using immunohistochemistry (IHC) and reverse transcription-polymerase 
chain reaction (RT-PCR) in gastric cancer. Summary background data The SN concept has recently been introduced in gastrointestinal tract cancers. The precise detection of lymph node metastasis including micrometastasis is important for SN navigation surgery. Methods Sixty-one patients with gastric cancer who were preoperatively diagnosed with T1-T2 (cT1-T2) and N0 (cN0) was enrolled. They underwent standard radical gastrectomy with lymph node dissection. One day before surgery, $4 \mathrm{mCi}$ of 99mTechnetium-tin colloid was endoscopically injected into the submucosa around the tumor. During surgery, radioisotope uptake in the lymph node was measured using Navigator GPS. All dissected lymph nodes were examined by RT-PCR in addition to Hematoxylin-Eosin (HE) staining and IHC. Results Sentinel nodes were identified in all patients $(100 \%)$. The incidences of metastasis determined by HE and IHC were $8.2 \%(5 / 61)$ and $13.1 \%(8 /$ 61), respectively. Micrometastases undetectable by IHC were identified in 14 patients $(23.0 \%)$ by RT-PCR. Only one patient had micrometastasis detectable by RT-PCR in lymph nodes other than $\mathrm{SN}$, but this patient had a cT 2 tumor. In patients with $\mathrm{cT} 1$ and $\mathrm{cN} 0$ tumors, the false negative and accuracy rates were $0 \%$ and $100 \%$, respectively. Conclusions Although the incidence of micrometastasis detected by RT-PCR was quite high, SN navigation identified such metastasis in all patients except one. Thus, the SN concept was applicable to patients with $\mathrm{cT} 1$ and cN0 gastric cancer, even when micrometastasis was detectable by RT-PCR.

\section{PD 7-5}

\section{$\mathrm{K}$-sam expression is associated with tumor progression and prognosis in gastric cancer}

Takahiro Toyokawa, Masakazu Yashiro, Kazuya Muguruma, Toru Inoue,Yoshito Yamashita, Tetsuji Sawada, Masaichi Ohira, Kosei Hirakawa

Department of Surgical Oncology, Osaka City University Graduate School of Medicine, Osaka, Japan

$\mathrm{K}$-sam gene is isolated as an amplified gene from a gastric cancer cell line, KATO-III. K-sam gene encodes a keratinocyte growth factor receptor which is associated with the proliferation of gastric cancer. In this study, we evaluated the expression of K-sam product in gastric cancer, and examined the relationships between this expression and various clinicopathologic characteristics. A total of 295 primary gastric tumor (160 early cancers and 135 advanced cancers) obtained from gastrectomy were investigated by staining them with a monoclonal antibody against K-sam product. Correlation between the expression of $\mathrm{K}$-sam and various clinicopathologic factors and prognosis were studied. Sixty-eight of $295(23 \%)$ samples were positive with $\mathrm{K}$-sam. There was a significant correlation between the expression of $\mathrm{K}$-sam and tumor depth $(P=0.003)$, lymph node metastasis $(P=0.01)$, peritoneal dissemination $(P=0.009)$, type of infiltration $(P=0.001)$. In advanced gastric cancer, there was a significant correlation between the K-sam expression and macroscopic findings $(P=0.001)$ and type of infiltration $(P=0.001)$. The prognosis of patients with $\mathrm{K}$-sam positive tumors was significantly worse compared with K-sam negative tumors (log rank; $P<0.001)$. In addition, multivariate analysis revealed that the $\mathrm{K}$-sam expression was a independent prognostic factor in advanced gastric cancer performed curative resection. These findings suggested that the expression of K-sam expression is associated with tumor progression and prognosis in gastric carcinoma.

\section{PD 7-6}

\section{HMGB1-inducible MIA is associated with nodal metastasis in oral squamous cell carcinoma}

Tomonori Sasahira ${ }^{1}$, Tadaaki Kirita ${ }^{2}$, Naohide Oue ${ }^{3}$, Ujjal K. Bhawal $^{1}$, Kazuhiko Yamamoto ${ }^{2}$, Wataru Yasui $^{3}$, Hiroki Kuniyasu ${ }^{1}$

${ }^{1}$ Department of Molecular Pathology, Nara Medical University, Kashihara, Japan, ${ }^{2}$ Department of Oral Surgery, Nara Medical University, Kashihara, Japan, ${ }^{3}$ Department of Molecular Pathology, Hiroshima University Graduates School of Biochemical Sciences, Hiroshima, Japan

Melanoma inhibitory activity (MIA) is an $11-\mathrm{kDa}$ secretory protein isolated from supernatants of the HTZ-19 malignant melanoma cells, which gene locus is mapped to chromosome 19q13.32-13.33. Previous reports indicate that MIA is correlated with invasion and metastasis in malignant melanoma, breast cancer, chondrosarcoma, glioma, and pancreatic cancer. HMGB1 is a chromosomal protein and is a RAGE ligand, which is overexpressed in oral squamous cell carcinoma (OSCC). HMGB1 is revealed to interact with NFkBp 65 to accelerate MIA expression. HMGB1 and NFkBp65 concurrently bind to a 30-bp region in the promoter region of the MIA gene designated as the highly conserved region. We examined MIA expression in 32 oral squamous cell carcinomas (OSCCs) by immunohistochemistry. Expression of MIA was observed in $50 \%$ of the cases (16/32). MIA immunoreactivity was not found in stage I and II cases, however, in stage III and IV cases, expression of MIA were 
observed in 54\% (7/13) and 69\% (9/13), respectively. A significant association was found between MIA immunoreactivity and histological metastasis to lymph nodes. In $3(19 \%)$ of 16 cases without nodal metastasis expressed MIA, whereas MIA expression was found in 13 $(81 \%)$ out of 16 cases with nodal metastasis $(P=0.028)$. MIA expression was also associated with expression of HMGB $1(P=0.023)$ and lymph vessel density $(P=0.031)$. In in vitro study, a metastatic human OSCC cell line, HSC-3 expressed MIA, HMGB1, and NFkBp65 at higher levels than nonmetastatic HSC-4 cells. By immunoprecipitation, HMGB1-NFkBp65 binding was significantly higher in HSC-3 cells than HSC-4 cells. HMGB 1-NFkBp65 binding levels in HSC-3 and HSC-4 cells were well correlated with those of MIA expression levels. These results suggest that HMGB1 upregulates MIA expression transcriptionally, which accelerates progression and metastasis in OSCC. MIA might be a marker for malignant potential of OSCCs.

\section{PD 8-1}

\section{Deregulated expressions of HOX genes in human colorectal cancer}

Jun-ichi Hamada, Minoru Takada, Yoko Takahashi, Mitsuhiro Tada, Tetsuya Moriuchi

Division of Cancer-Related Genes, Institute for Genetic Medicine, Hokkaido University, Sapporo, Japan

HOX genes are known not only as master genes to control the morphogenesis but also as regulator genes to maintain tissue- or organ-specificity in adult body. We have reported that HOX gene expressions are dysregulated in a variety of solid tumors such as melanoma, breast, or lung and gastric cancers. In this study, we compared the HOX expression patterns between cancerous and non-cancerous of colorectal cancers. The expression levels of 39 HOX genes in 37 human colorectal cancer tissues and 20 non-cancerous mucosa tissues were quantified by the real-time RT-PCR method. In non-cancerous mucosa, the expression levels of HOXB6 and B9 in left large intestine (descending and sigmoid colon, and rectum) were significantly lower than those in right large intestine (cecum, ascending and traverse colon) whereas the expression levels of HOXB13 and D13 in the left were higher than those in the right $(P<0.01$, Mann-Whitney $U$-test $)$. Ten HOX genes were expressed differentially between cancer and non-cancerous tissues in left large intestine $(P<0.01)$. In right large intestine, the expression of only $2 \mathrm{HOX}$ genes was different $(P<0.01)$. The primary colorectal cancer tissues with hepatic metastasis showed higher expression of HOXC 12 than those without hepatic metastasis $(P<0.01)$. In comparison between the primary and hepatic metastatic sites, metastatic tissues showed lower expression of HOXD8 than the primary tissues $(P<0.01)$. These results suggest that the expression pattern of HOX genes in colorectum reflects its developmental origin and that the disordered expressions of HOX genes are involved in carcinogenesis and malignant progression.

\section{PD 8-2}

\section{Characterization of breast cancer metastasis suppressor 1 (BRMS1) in cell strains from MCF10A}

Douglas Hurst, Blake Moore, Alka Mehta, Kevin Nash, Danny Welch

University of Alabama at Birmingham, USA

Breast cancer metastasis suppressor 1 (BRMS1) suppresses metastasis without blocking tumorigenesis in multiple human and murine cancer cells of diverse origins. The molecular mechanisms of BRMS1 are not well understood and thus far, mechanistic studies of BRMS1 have been limited to systems in which BRMS1 was reexpressed in metastatic cell lines. In order to study the role of BRMS1 in the progression of breast cancer, the genetically related cell lines representing premalignant breast disease (MCF10A and MCF10AT) and metastatic carcinoma (MCF10CA a.1 and MCF10CA d.1 alpha) were analyzed. Endogenous BRMS1 protein was localized to the nucleus with no significant difference in levels of expression. However, mRNA levels were probed by real-time RT-PCR and found to be higher in the metastatic cell lines. This may be partially explained by alternative splice variants that were detected by RT-PCR and found to be differentially expressed in these cell lines and suggests that only select variants are involved in metastasis regulation. BRMS1 has been previously shown to interact with transcriptional regulatory protein complexes. To analyze protein interactions with endogenous BRMS1, interactors were immunopurified with an affinity column generated with a BRMS1 antibody and identified by mass spectroscopy. A component of the TFIID transcriptional complex, the TATA-binding protein, was identified as a novel interactor of BRMS1. This implicates BRMS1 in cell cycle regulation of transcription and supports our overall hypothesis that BRMS1 functions as a transcriptional regulator to suppress metastasis. Support: CA87728 (DRW), F32CA113037 (DRH), CA89019 (DRW), National Foundation for Cancer Research (DRW) 


\section{PD 8-3}

\section{Activation states of RhoA in human small cell lung cancer cells: contribution of $G$ protein coupled receptors}

Hirokazu Touge, Hiroki Chikumi, Tadashi Igishi, Jun Kurai, Haruhiko Makino, Yoshisato Tamura, Miyako Takata, Kazuhiko Yoneda, Masaki Nakamoto, Hisashi Suyama, Eiji Shimizu

Division of Medical Oncology and Molecular Respirology, Department of Multidisciplinary Internal Medicine, Faculty of Medicine, Tottori University, Tottori, Japan

Small cell lung cancer (SCLC) cells have high metastatic potentials. These properties might be a consequence of the high expression or activation states of RhoA. Former studies reported that RhoA overexpression contributes to human cancer development including SCLC, but the activation states of RhoA in these cancer cells are poorly defined. In this study, we examined both the expression levels and the activation states of RhoA in SCLC cells by quantitative real-time reverse transcriptase-polymerase chain reaction and in vivo Rho guanine nucleotide exchange assay, respectively. Moreover, we dissected the signaling pathway from the cell surface receptors to RhoA using a broad-spectrum $G$ protein coupled receptor (GPCR) antagonist. We found that four of six small cell lung carcinoma (SCLC) cell lines exhibited a moderate to high activation rate of RhoA, whereas only one cell line exhibited high RhoA expression. The addition of the GPCR antagonist, [D-Arg ${ }^{1}$, D-Trp ${ }^{5,7,9}$, Leu ${ }^{11}$ ] Substance $\mathrm{P}$ (SP), reduced RhoA activity by almost $60 \%$ in $\mathrm{H} 69$ SCLC cells. These results suggest that RhoA is activated in SCLC cells independent of its expression levels, and the high activation state of RhoA in SCLC cells mainly depends on a neuroendocrine peptide autocrine system which signals through GPCR to RhoA. This study may help in developing novel therapeutic strategies against SCLC.

\section{PD 8-4}

\section{The expression of apoptosis mediator proteins in 173 colorectal liver metastases and their case matched corresponding primaries}

Mark T. Swinscoe ${ }^{1}$, J. Lance Burn ${ }^{1}$, Jonathan P. Bury ${ }^{2}$, Iain C. Cameron ${ }^{1}$, Ali W. Majeed ${ }^{1}$, Nigel C. Bird ${ }^{1}$

${ }^{1}$ Liver Research Group, University of Sheffield,
Sheffield, England, UK, ${ }^{2}$ Department of Histopathology, University of Sheffield, Sheffield, UK

Introduction Half of all cases of primary colorectal cancer result in metastases within the liver. Dysregulation of apoptosis is a potential mechanism that could result in uncontrolled cell growth. Tumour cells expressing certain apoptosis inducing ligands are believed to evade immune attack. The expression of apoptosis mediating proteins in both colorectal primary tumours and their corresponding liver metastases was studied on a large scale to assess their influence on biological aggressiveness. Methods The differential expression of apoptosis mediating proteins was investigated in 173 pairs of primary colorectal carcinomas and the corresponding liver metastases from the same patient. The expression of FasL, Bcl-2 and Bax proteins was determined by immunohistochemistry using a tissue microarray containing samples of the primary carcinoma, liver metastasis, metastasis tumour interface with the liver and uninvolved liver in 173 cases. Tissue immunoreactivity was assessed following incubation with primary monoclonal antibodies FasL (clone G247-4, BD Pharmingen), Bcl-2 (clone 124, Dako) and Bax (clone 2D2, Abcam) and with the Dako CSA II amplification system (FasL, Bcl-2) or HRP-steptavidin (Bax) visualised with diaminobenzidine (DAB, Vector labs). The samples were scored for percentage of positively stained tumour cells and intensity of staining by two independent observers and scores correlated with parameters of clinical outcome. Results FasL protein was highly expressed in both primary carcinomas and liver metastases. However, significantly fewer metastases were highly FasL positive ( $72 \%$ vs. $52 \% ; P<0.001$, Chi square). High FasL expression in liver metastases ( $>75 \%$ positive tumour cells) was associated with a reduced median survival compared with FasL negative metastases (37.1 months vs. 28.5 months). Bcl-2 expression was demonstrated in both primary carcinomas and liver metastases with no significant difference in the number of positive cases $(61 \%$ vs. $52 \%$, NS). Similarly, Bcl-2 expression in metastases conferred no difference in median survival when compared with non $\mathrm{Bcl}-2$ expressing metastases (36.2 vs. 36.5). Most primaries and metastases failed to express Bax with no significant difference in Bax positive cases ( $27 \%$ vs. $23 \%$, NS). Metastases that expressed Bax were associated with a reduced median survival (27.4 months vs. 37.2 months). Conclusions Colorectal liver metastases express the Fas ligand less frequently than primary carcinomas. Furthermore, FasL expression in liver metastases appears to have a negative impact on patient survival. This supports the hypothesis that Fas mediated apoptosis enables escape from immune surveillance. Similarly, Bax expression in metastases appears to reduce 
survival however, expression of Bcl-2 in metastases does not influence patient survival.

\section{PD 8-5}

\section{High expression of h-prune in gastric cancer with gastric phenotype : expression of $h$-prune is associated with poor survival in gastric cancer}

\section{Naohide Oue ${ }^{1}$, Akira Kikuchi ${ }^{2}$, Wataru Yasui ${ }^{1}$ \\ ${ }^{1}$ Department of Molecular Pathology, Hiroshima University Graduate School of Biomedical Sciences, Hiroshima, Japan, ${ }^{2}$ Departments of Biochemistry, Hiroshima University Graduate School of Biomedical Sciences, Hiroshima, Japan}

$\mathrm{H}$-prune has been identified as a glycogen synthase kinase 3 (GSK-3)-binding protein and has been suggested to be involved in cell migration. In the present study, immunohistochemical analysis of h-prune was performed in various human tumors $(n=277)$. In addition, because h-prune physically interacts with $\mathrm{nm} 23-\mathrm{H} 1$, a metastasis suppressor gene, we performed immunostaining of nm23$\mathrm{H} 1$ in gastric cancer (GC). H-prune was weekly expressed in non-neoplastic gastric, colorectal, and pancreatic tissues. Among 143 GCs, 92 colorectal cancers (CRCs), and 42 pancreatic cancers (PCs), high expression of h-prune was detected in $43(30 \%), 27(29 \%)$, and $18(43 \%)$, respectively. Expression of h-prune was significantly associated with advanced $\mathrm{T}$ grade (depth of tumor invasion), $\mathrm{N}$ grade (degree of lymph node metastasis), and tumor stage in GC, CRC, and PC, respectively. Furthermore, h-prune expression was associated with poor survival in advanced GC cases $(P=0.0102)$. nm $23-\mathrm{H} 1$ was expressed in almost all $(87 \%) \mathrm{GC}$, and there was no clear correlation between expression of $\mathrm{nm} 23-\mathrm{H} 1$ and hprune. Expression of the gastric and intestinal phenotypes of GC was examined by immunostaining for MUC5AC, MUC6, MUC2, and CD10. Of 60 GCs showing the gastric phenotype, high expression of h-prune was observed in $26(43 \%)$, whereas in the remaining $83 \mathrm{GC}$ cases, high expression of h-prune was observed in $17(20 \%)(P=0.0053)$. These results suggest that highly expressed h-prune could be related to higher tumor aggressiveness. High expression of h-prune may be involved in GCs with a gastric phenotype.

\section{PD 8-6}

\section{Significance of cancer metastasis to the pulmonary ligament in the pathogenesis of cancerous pleural effusion}

Hisashi Oshiro ${ }^{1}$, Yoshiaki Inayama ${ }^{1}$, Shoji Yamanaka ${ }^{1}$, Isao Yoshihama ${ }^{2}$, Motoshige Kudo ${ }^{3}$, Yoshiro Ebihara ${ }^{3}$

${ }^{1}$ Division of Anatomic and Surgical Pathology, Yokohama City University Hospital, Kanagawa, Japan, ${ }^{2}$ Electron Microscopy Laboratory, Tokyo Medical University, Tokyo, Japan, ${ }^{3}$ Department of Pathology, Tokyo Medical University, Tokyo, Japan

Background It is well known that cancerous pleural effusion could occur despite no evidence of pleural dissemination or extrapleural direct invasion of cancer cells. One of the hypotheses is that cancer cells can leak from lymphatic stomata on the serosal surface which open lymph capillary directly to body cavity. We have previously demonstrated that lymphatic stomata could be observed on the surface of the pulmonary ligament at the microscopic level in human thoracic cavity, and cancer metastasis to the pulmonary ligament might play a role in the pathogenesis of pleural carcinomatosis. The aim of this study was to elucidate the significance of metastasis to the pulmonary ligament in the pathogenesis of cancerous pleural effusion. Methods Cases with solid cancers originated from any organ, including carcinomas and sarcomas except for lymphomas and mesotheliomas, were retrieved from autopsy records of our hospitals between 2000 and 2005, and 31 cases were available for investigation of both pleural effusion and whole body. Cancerous pleural effusion was cyotologically examined by glass slides of Papanicolaou stain, Giemsa stain, periodic acid-Schiff stain, and alcian blue stain. If available, cell block samples of buffy coat from the pleural effusion were used for pathological examination. Pleural dissemination was defined as at least 2 lesions of histopathologically demonstrable cancer cells on the pleural surface of the thoracic cavity except for the portion of pulmonary ligament. Pulmonary ligament and venous angle lymph node were also histopathologically examined in addition to routine autopsy investigations. Lymphatic stomata on the surface of the pulmonary ligament were observed by light microscope and scanning electron microscope. Findings A large number of lymphatic stomata were found in pulmonary ligament at the microscopic level in all cases. Nine cases had pleural dissemination, which also had cancerous pleural effusion and metastasis to the pulmonary ligament and venous angle lymph node. Thirteen out of the remaining 22 cases without pleural dissemination were positive for cancerous pleural effusion and metastasis to the pulmonary ligament and venous angle lymph node. Regardless of the presence or absence of pleural dissemination, all cases with cancerous pleural effusion demonstrated metastasis to the lymphatic stomata of the pulmonary ligament. Interpretation 
Our findings strongly suggest that cancer metastasis to the pulmonary ligament plays an integral role in the pathogenesis of cancerous pleural effusion in cases without pleural dissemination or extrapleural direct invasion of cancer cells.

\section{PD 8-7}

\section{Proliferative activity of the sentinel lymph node in gastric cancer}

Shigehiro Yanagita, Shoji Natsugoe, Yoshikazu Uenosono, Tsutomu Kozono, Katsuhiko Ehi, Takaaki Arigami, Hideo Arima, Hiroshi Higashi, Akihiro Nakajo, Sumiya Ishigami, Shuichi Hokita, Takashi Aikou

Department of Surgical Oncology and Digestive Surgery, Field of Oncology, Course of Advanced Therapeutics, Kagoshima University Graduate School of Medical and Dental Sciences, Kagoshima, Japan

Background According to the sixth edition of the TNM classification, the issues of sentinel node ( $\mathrm{SN}$ ) and micrometastasis and isolated tumor cells (ITC) were newly defined. The purpose of the present study was to examine the frequency of these metastases and to examine their proliferative activity, with a specific focus on SNs of gastric cancer. Methods A total of 133 patients with cT1-2 tumor (cT1: 104, cT2: 29) with sentinel node mapping were enrolled in this study. Lymph node metastases, including micrometastasis and ITC, were examined by routine histological examination and immunohistochemical staining with anticytokeratin (AE1/AE3). Primary tumor and lymph node metastases were detected with the Ki-67 antibody so that proliferative activity could be evaluated. Results The number of patients and sentinel lymph node metastases, including micrometastasis and ITC, were $21(16 \%)$ and 52, respectively. The frequencies of metastasis, micrometastasis and ITC were $48 \%, 25 \%$ and $27 \%$, respectively. Ki-67 expression correlated well with lymphatic invasion $(P=0.0001)$, venous invasion $(P<0.0001)$, and lymph node metastasis $(P<0.0001)$. The Ki-67 positive cells were found in $96 \%$ of metastases, $92 \%$ of micrometastases and $29 \%$ of ITCs. Conclusions As lymph node metastases, including micrometastasis and ITC in SNs have proliferative activity, it is important that these tiny cancer cells or clusters be diagnosed and addressed surgically for proper treatment of gastric cancer.

\section{PD 8-8}

Genetic and epigenetic profiling in early colorectal tumors and prediction of metastatic potential in stage I (pT1) colorectal cancers

Hiroaki Taniguchi ${ }^{1}$, Hiroyuki Yamamoto ${ }^{1}$, Yasushi Adachi $^{1}$, Kohzoh Imai ${ }^{2}$, Yasuhisa Shinomura ${ }^{1}$

${ }^{1}$ First Department of Internal Medicine, Sapporo Medical University School of Medicine, Sapporo, Japan, ${ }^{2}$ Sapporo Medical University, Sapporo, Japan

Although RAS-RAF and Wnt signaling pathways are known to play an important role in colorectal cancers (CRCs), little is known about their role in early colorectal tumors. We analyzed the frequencies of genetic and epigenetic alterations of those pathways in early colorectal tumors (including flat-type tumors) and the relationship between those alterations and invasion metastasis of pT1 CRCs. In 307 early colorectal tumors, we investigated genetic and epigenetic alterations by using direct DNA sequencing and real-time PCR to measure DNA methylation (MethyLight), respectively. Methylations of RASSF2, MGMT, WIF-1, EPHB2, CDKN2A and MLH1 were detected in $44.3 \%, 30.3 \%$, $81.4 \%, 7.5 \%, 43.6 \%$ and $13.4 \%$ of the 307 early colorectal tumors, respectively. Mutations of KRAS, BRAF, PIK3CA and $\beta$ catenin were detected in $25.4 \%$, $4.6 \%, 1.6 \%$ and $9.4 \%$ of those tumors, respectively. WIF-1 methylation was detected in a significantly higher percentage of protruded-type tumors than in flattype tumors. Mutation of at least one gene was detected in a significantly higher percentage of flat-type tumors than in protruded-type tumors. RASSF2 methylation was correlated significantly with KRAS or BRAF or PIK3CA mutation. Multiple logistic analysis showed that lymphatic invasion and RASSF2 methylation with KRAS or BRAF or PIK3CA mutation were independent risk factors for venous invasion in pT1 CRCs. In conclusion, since genetic alterations of RAS-RAF and Wnt signaling pathways have frequently occurred in flat-type tumors, they seem to have a distinct genetic profile different from that of protruded-type tumors. RASSF2 methylation with oncogenic activation is a promising biomarker for predicting metastatic potential of pT1 CRCs.

\section{PD 8-9}

\section{DNA microarray analysis of chemokine genes related cancer metastasis}

Hironobu Yanagie ${ }^{1}$, Tomoyuki Hisa ${ }^{1}$, Takuya Tamatani $^{2}$, Kayo Shimizu ${ }^{3}$, Nao Nitta ${ }^{2}$, Toshio Yamori $^{4}$, Isao Osada ${ }^{5}$, Teturo Nishihira ${ }^{6}$, Shushi 
Higashi $^{7}$, Hiroki Seguchi ${ }^{7}$, Yoshikazu Ota ${ }^{7}$, Masayasu Shima $^{7}$, Shiro Kanegasaki ${ }^{2}$, Katsuji Matsushima ${ }^{8}$, Masazumi Eriguchi ${ }^{1}$

${ }^{1}$ Dept. of Intellectual Property, Project of Inhibition of Cancer Metastasis. RCAST, The University of Tokyo, Tokyo, Japan, ${ }^{2}$ Effector Cell Institute, Ltd. Co., Tokyo, Japan, ${ }^{3}$ Kaken-Genecs, Ltd. Co., Chiba, Japan, ${ }^{4}$ Dept. of Molecular Pharmacology, Cancer Institute, Tokyo, Japan, ${ }^{5}$ Dept. of Surgery, Shin-Yamanote Hospital, Tokyo, Japan, ${ }^{6}$ Dept. of Surgery, Fukujuji Hospital, Tokyo, Japan, ${ }^{7}$ Dept. of Surgery, Miyakonojyo Medical Association Hospital, Miyazaki, Japan, ${ }^{8}$ Dept. of Molecular Prevent Medicine, Graduate School of Medicine, The University of Tokyo, Tokyo, Japan

In order to clarify the relationship between expression of chemokine receptors on cancer cells and metastasis to several organs, we performed the DNA microarray analysis using RNA of cancer cell lines and clinical samples with DNA chips specific to chemokines, chemokine receptors. The clinical samples were obtained with permission of Hospital Ethics Committee and patient's informed consent. Upregulated genes of chemokine in gastric cancer cell lines were SLC, RANTES, BRAK, MCP-1, MCP-3 alpha, GRO, GRO3, IL-8, NAP-2, SDF-1 alpha, SDF-1 beta, and downregulated genes of chemokine were MIP-1 alpha, TECK. The upregulated gene of chemokine receptor was CCR-6. Upregulated genes of chemokine in colon cancer cell lines were ILO, MCP-1, MCP-4, MIP-1 beta. The secretion of MIP1 was increased in the MKN-74 and KATO-III gastric cancer cell lines, but the secretion of MIP-1 alpha was suppressed. Upregulated genes of chemokine in colon cancer patients were SPP1, IL-27 RA, CXCL-3, CXCL-1. These results showed that cancer cells easily constructed the edematous and inflammatory circumstances in the cancer environments and to metastase another organs with the suppression of dendritic cells and escape the surveillance of tumor immunity.

\section{PD 8-10}

\section{Cancer-death related genomic aberration of esophageal squamous cell carcinoma revealed} by array-based comparative genomic hybridization

Shigeo Hirasaki ${ }^{1,2}$, Hiroshi Inoue ${ }^{1}$, Tsuyoshi Noguchi ${ }^{3}$, Takashi Hirano ${ }^{4}$, Kenichi Sugihara ${ }^{2}$, Masaki Mori ${ }^{1}$

${ }^{1}$ Department of Surgery, Medical Institute of Bioregulation, Kyushu University, Beppu, Oita, Japan,
${ }^{2}$ Department of Surgical Oncology, Graduate School of Medicine, Tokyo Medical and Dental University, Tokyo, Japan, ${ }^{3}$ Department of Oncological Science (Surgery II), Faculty of Medicine, Oita University, Yufu, Oita, Japan, ${ }^{4}$ Gene Function Research Center, National Institute of Advanced Industrial Science and Technology (AIST), Tsukuba, Ibaraki, Japan

Purpose The prognosis of patients with esophageal carcinoma is poor. To identify the genomic regions that relate cancer death, we analyzed the whole-genomic aberration profiles of esophageal squamous cell carcinomas (ESCCs) from 19 tumors using array-based comparative genomic hybridization (aCGH). Methods Nineteen post-operative ESCC samples were prepared for this study. All 19 patients were followed up at least four- and a half years. Each sample was laser microdissected to obtain pure cancer cell populations, and then genomic DNAs were extracted. The extracted DNAs were analyzed by aCGH that contains 4,030 spots of BAC clones. Results With an overview of all samples, the distinctive aberrations were disclosed at some regions. One of the most representative region was $1.1 \mathrm{Mb}$ gain at 11q13.3 which was reported previously. On the other hand, some novel aberrations such as loss of 11 p 15.4 , or 16 p 13.3 were disclosed. Nine patients survived, and 10 patients died of disease recurrence after the operation. When compared both groups, a significant differences was recognized at 82 of 4,030 spots. And almost a half of these spots were presented at some specific regions in 4q, 5p, 8q, 13q, 18p, and Xq. We analysed these 82 spots by Kaplan-Meier method and a significant difference was recognized in 33 spots by LogRank test. Conclusion Our aCGH data revealed the distinctive regions that relate postoperative death, and this may be the first study to clarify the cancer-related regions by using high-resolutional aCGH method. Genomic gain or loss may not always result high or low gene/protein expression of that region, respectively. When we can find the regions that strongly correlate with the postoperative prognosis, it may be very useful to determine the therapeutic strategy.

\section{PD 9-1}

\section{Immune evasion: a requirement for breast cancer metastasis?}

Bradley N. Bidwell ${ }^{1,2}$, Belinda S. Parker ${ }^{1}$, Robin L. Anderson ${ }^{1,3}$

${ }^{1}$ Peter MacCallum Cancer Centre, East Melbourne, Victoria, Australia, ${ }^{2}$ Department of Biochemistry and 
Molecular Biology, The University of Melbourne, Melbourne, Victoria, Australia, ${ }^{3}$ Department of Pathology, The University of Melbourne, Melbourne, Victoria, Australia

The 4T1.2 murine model of breast cancer is a syngeneic metastasis model in which primary mammary carcinomas spontaneously metastasise to the lymph nodes, lungs and bone. We have utilised a novel immunopurification technique to isolate tumour epithelial cells from the primary tumour and from matched spine metastases. To obtain a gene expression signature that represents changes involved in the establishment of a metastasis in the bone microenvironment, we used the Affymetrix platform to compare the gene expression profiles of the tumour cells isolated from primary tumours and bone metastases. An analysis of the gene ontology categories of the differentially expressed genes was conducted and the three most significant functional groups were immune response, defense response and response to biotic stimulus: three categories representing immune signalling and induction. We then analysed the $5^{\prime}$ UTRs of the genes from our analysis that were included in these three categories and discovered that a significant proportion share a similar regulatory sequence. The shared sequence is a target for an interferon family transcription factor which was also identified in this analysis as being downregulated in metastatic epithelial cells. These data provide strong evidence that modulation of the immune system is a critical element in the development of bone metastases. It is possible that the metastatic tumour cells need to adapt to evade attempts by the immune system to eliminate them from an the new bone environment. This would lend strength to the theory that the development of micrometastases into macrometastases is the rate limiting step of metastasis, as constant elimination of unsuccessful micrometastases by the immune system would prevent the development of large numbers of macrometastases.

\section{PD 9-2}

\section{Interaction of cancer cells with glial cells in microenvironment of brain metastasis}

Iguchi Haruo $^{1}$, Seike Toshihiro ${ }^{2}$, Fujita Kyota ${ }^{2}$, Kido Mizuho $^{3}$, Tanaka Teruo ${ }^{3}$, Noda $\mathrm{Mami}^{2}$

${ }^{1}$ Clinical Research Institute, Shikoku Cancer Center, Japan, ${ }^{2}$ Laboratory of Pathophysiology, Graduate School of Pharmacy, Kyushu University, Japan, ${ }^{3}$ Department of Oral Anatomy and Cell Biology, Graduate School of Dental Science, Kyushu University, Japan
Incidence of brain metastasis has been increasing in the recent years, however, its molecular mechanism is poorly understood to date. In cancer metastasis, it is important for cancer cells to interact with stromal cells in its microenvironment, which makes it possible for cancer cells to survive and proliferate at the metastatic site. We therefore examined interaction of cancer cells with glial cells in an experimental model of brain metastasis using human lung cancer cells (HARA-B). Brain metastases were histologically proven $4-5$ weeks after intracardiac inoculation of HARA-B cells in nude mice. Accumulation of activated microglia and astrocytes were found around metastatic lesions in the brain. The number of microglia rather decreased whereas that of astrocytes showed marked increase along with an increase in size of metastatic foci. We also performed co-culture of HARA$B$ cells with microglia and/or astrocytes, which were isolated from mouse brain, in vitro. Co-culture and/or conditioned medium of microglia suppressed the growth of HARAB cells, whereas those of astrocytes enhanced it. Several inflammatory cytokines (i.e. IL-1beta, TNF-alpha, IL-6) were identified in the conditioned medium of astrocytes by ELISA, and expression of receptors for these cytokines on HARA-B cells was confirmed by immunohistochemistry. These results suggest that glial cells, which migrate around metastatic foci, affect tumor progression through inflammatory cytokines in the microenvironment of brain metastasis. Astrocytes might be a molecular target to control brain metastasis.

\section{PD 9-3}

\section{P-glycoprotein inhibitor overcomes paclitaxel resistance of brain metastasis via targeting of the tumor-associated endothelial cells}

\author{
Do-Hyun Nam ${ }^{1}$, Myung Jin Son ${ }^{1}$, MI Hyun Kim ${ }^{1}$, \\ Gwan Sun Lee ${ }^{3}$, Maeng Sup Kim ${ }^{3}$, Sang Yongq Song ${ }^{2}$, \\ Kwan Park ${ }^{1}$
}

${ }^{1}$ Dept. of Neurosurgery, Samsung Medical Center, Sungkyunkwan Univ School of Medicine, Seoul, Korea, ${ }^{2}$ Dept. of Pathology, Samsung Medical Center, Sungkyunkwan Univ School of Medicine, Seoul, Korea, ${ }^{3}$ Hanmi Pharm. Co., Seoul, Korea

Studies have been undertaken to determine usefulness and mechanism of action of p-glycoprotein inhibitors (pGPIs) to overcome drug resistance in brain metastasis. We focused on the role of tumor associated endothelial cells (TAECs) in the chemotherapy using paclitaxel, good pGP substrate, with pGP and pGPI interaction models. With the rhodamine 123 efflux assay, we validated the effectiveness of novel pGPI 
(HM30181A) and XR 9576 (tariquidar) as well as evaluate the function of pGP in tumor cells (K1735, mouse melanoma cell) and brain endothelial cells (Bend3, mouse brain EC). Expression of pGP in cells were evaluated with immunohistochemistry (IHC) and RT-PCR. In vitro cytotoxicity assay of paclitaxel was also evaluated in K1735 cells and Bend 3 cells according to the addition of the pGPIs. After inoculation of the k1735 cells into $\mathrm{C} 3 \mathrm{H}$ mice subcutaneous tissue and brain, we colocalized the expression of CD31 and pGP as well as the response of paclitaxel metronomic chemotherapy (200 $\mu \mathrm{g}$ IP per $\mathrm{C} 3 \mathrm{H}$ mice, two times a week) according to the addition of pGPI (HM30181 A, $400 \mu \mathrm{g}$ PO, each time of paclitaxel IP). According to IHC and RT-PCR, K1735 does not express the pGP, but Bend3 expresses pGP. Rhodamine efflux assay showed that pGPIs inhibited the rhodamine 123 efflux only in Bend3 cells. IC50 of paclitaxel is more than $5000 \mathrm{nM}$ in bEnd.3 and is $19.59 \mathrm{nM}$ in K1735, however, HM30181A dramatically reduced IC 50 value of paclitaxel by $96 \%$ at $0.1 \mathrm{nM}$ relative to control in bEnd.3, but no effect in K1735. Subcutaneous ECs and TAECs do not express pGP, however, brain ECs as well as brain TAECs expresses pGP in vivo. There was significant reduction of tumor size by metronomic treatment of paclitaxel in subcutaneous melanoma mice model, however, no response in brain melanoma. Addition of HM30181A to paclitaxel showed marked reduction of tumor size in brain melanoma, however, no additional effect in subcutaneous melanoma bearing mice. Inhibition of pGP function at brain tumor associated EC restored the chemosensitivity to paclitaxel based vascular targeted treatment. Our data suggest that organ or tumor specific endothelial survival mechanism is crucial for the vascular targeted treatment especially in brain metastasis which have potent and multiple survival factors.

\section{PD 9-4}

\section{KISS1 secretion is required for multiple organ metastasis suppression}

Kevin Nash, Pushkar Phadke, Kedar Vaidya, Douglas Hurst, John Kappes, Danny Welch

\section{University of Alabama-Birmingham, USA}

Although the KISS1 metastasis suppressor has been shown to play an important role in the regulation of the metastatic phenotype in human cancers and in in vivo mouse models, little is known about its mechanism. Recent evidence suggests that KISS1 may be processed and secreted but it is unclear whether these events are necessary for its anti-metastatic effects. In the present study, we tested the hypothesis that KISS1 secretion is required for multiple organ metastasis suppression using the metastatic human melanoma cell line C8161.9. To detect secretion, KISS1 was engineered with an internal FLAG epitope in order to identify processed forms in the absence of a suitable antibody. Green fluorescent C8161.9 cells were stably transfected with pcDNA 3-FLAG KISS 1 (KFM) or pcDNA 3-FLAG KISS 1 lacking the putative secretion signal (KFMdeltaSS). Secreted KISS1 proteins were immunoprecipitated from conditioned medium and detected using FLAG M2 antibody. Despite equal KISS1 protein expression in whole cell lysate for both constructs, KISS1 secretion and processing was only detectable for KFM. The importance of KISS1 secretion for metastasis suppression was determined in vivo using three stable C8161.9 KFM or KFMdeltaSS clones. Cells $\left(2 \times 10^{\mathrm{e} 5}\right)$ were injected into the left ventricle of athymic mice and organs were removed after 5 weeks to assess the incidence and frequency of metastatic disease using fluorescent microscopy. All three secreting KFM clones suppressed the incidence and frequency of metastatic disease in the lung, kidney, eye and bone by 56-100\% when compared to vector control ( $P$ less than 0.001$)$. In stark contrast, all three KFMdeltaSS clones failed to reduce the significance or frequency of metastatic disease in these organs. These data indicate that KISS1 secretion is necessary for metastasis suppression in multiple organs opening the possibility for administration of exogenous mimetics for anti-metastatic therapy. Support: CA87728, T32 GM08361, DAMD17-02-10541, National Foundation for Cancer Research, CA87728, F32CA 113037, KomenPDF1122006, and P30-CA13148.

\section{PD 9-5}

\section{Gene expression profiles from hepatocytes at the tumour margin in colorectal liver metastases}

Nigel C. Bird ${ }^{1}$, Lance Burn ${ }^{1}$, David Mangnall ${ }^{1}$, Ali W. Majeed $^{2}$

${ }^{1}$ Liver Research Group, University of Sheffield, Sheffield, UK, ${ }^{2}$ Sheffield Hallam University, Sheffield, UK

Introduction Little data is available on the means by which colorectal liver metastases invade the liver, although early reports suggest that the process is different to that of the corresponding primary tumour [1]. Liver metastases are a heterogeneous group of tumours with differing growth patterns which are associated with different survival times. Using cDNA microarray chips, we have examined gene expression in "pushing" and desmoplastic tumour margins, two of the most common 
patterns found in liver metastases. Methods Fully informed consent was obtained from patients admitted for liver surgery for colorectal cancer metastasis (South Sheffield Ethics Committee SS/00/342). Tissue was obtained at operation from the tumour:liver interface and normal liver, distant to the tumour. After dissection, samples were snap frozen and stored in liquid nitrogen until analysis. Twelve $\mu \mathrm{m}$ sections were cut on a cryostat and laser capture microdissection (Arcturus, Herts, UK) was used to obtain individual hepatocytes from areas adjacent to the metastasis and from separate normal liver samples. Total RNA was extracted from these using Trizol reagent (Gibco Life Technologies, Scotland), RNA integrity and concentration were analysed using an Agilent 2100 Bioanalyser. Affymetrix (Santa Clara, USA) HG-U133 Plus 2.0 chips, representing 38500 genes, were used to examine gene expression. Data analysis was performed using two software packages, Array Assist and Pathway Architect (Iobion, La Jolla, USA). Results Two groups of samples from 6 patients were studied, 3 tumours with a pushing margin and 3 with a desmoplastic margin. Differential gene expression profiles for each group was obtained by comparing expression levels between normal hepatocytes and hepatocytes taken from the tumour margin. Analysis of differential gene expression was carried out using an unpaired $t$-test with cutoff levels of a 2-fold change in expression (up or down) compared to normal tissue, with a probability of less than 0.05 . In the pushing group there were 135 significantly different probe sets (79 downregulated), with 12 downregulated extra-cellular matrix genes and 8 downregulated cytoskeletal genes. In the desmoplastic group there were 46 significant genes ( 20 downregulated); 3 downregulated serine endoprotease inhibitors and 2 upregulated cytoskeletal genes. Conclusion Statistically significant differences in gene expression are present in hepatocytes adjacent to colorectal liver metastases when compared with normal hepatocytes. In tumours with a pushing margin there is significant down-regulation of genes associated with extra-cellular matrix turnover; desmoplastic tumours showed downregulation of serine endopeptidases.

\section{Reference}

1. Illemann M, Bird NC, Majeed AW et al (2006) Mol Cancer Res 4(5):293-302

\section{PD 9-6}

\section{Differential signaling responses as a potential}

mechanism for BRMS 1 mediated metastasis suppression

Kedar Vaidya ${ }^{1}$, Sitaram Harihar ${ }^{2}$, Pushkar Phadke ${ }^{1}$, Daryll DeWald ${ }^{2}$, David Hicks ${ }^{3}$, Graham Casey ${ }^{3}$, Danny Welch ${ }^{1}$

${ }^{1}$ University of Alabama-Birmingham, USA, ${ }^{2}$ Utah State University, ${ }^{3}$ Lerner Research Institute, USA

The BRMS1 metastasis suppressor inhibits metastasis of breast cancer and melanoma cell lines without blocking tumorigenesis. Previous studies indicate that BRMS1 associates with histone deacetylases and may control gene regulation. In addition, BRMS1 also affects signaling cascades, including selective reduction of phosphatidylinositol 4,5-bisphosphate levels and the activation/translocation of NF-kappa B. The aim of this study was to test the hypothesis that BRMS1 interferes with downstream transmission of external mitogenic stimuli that ultimately leads to metastasis suppression. For this study, MDA-MB-231/435 breast cancer cells and their BRMS 1 transfectants (231BRMS1/ 435BRMS1) were used. 231BRMS1/435BRMS1 or vector transfectants were exposed to epidermal growth factor (EGF, $0-50 \mathrm{ng} / \mathrm{ml}$ ) and downstream effectors were studied using Western blot analysis. EGF stimulated downstream AKT-Ser 473 phosphorylation in both 231- and 435-vector controls, however, phosphorylation was markedly $(>50 \%)$ reduced in 231BRMS1 and completely abolished in 435 BRMS1. EGF failed to modulate p42/p44 MAPK phosphorylation in 231 and 231BRMS1; whereas, 435BRMS1 showed marked reduction in MAPK phosphorylation compared to 435 cells. Upstream analysis revealed that 231BRMS1 had decreased EGF-receptor protein as well as mRNA, showing an inverse dose-response to BRMS1. EGFR expression in 435BRMS1 was undetectable. 231BRMS1/435BRMS1 cells failed to show Factin reorganization following EGF treatment (30 and $60 \mathrm{~min}$ ) further supporting the hypothesis that BRMS 1-expressing cells were less responsive to external signals. 231BRMS1 failed to show F-actin reorganization following PDGF stimulation even though PDGF receptor levels remained unchanged, indicating a deficit in signal transmission. Further, insulin $(100 \mathrm{ng} / \mathrm{ml})$ stimulated AKT phosphorylation but not of p42/p44 MAPK and phorbol ester $(100 \mathrm{nM})$ stimulated phosphorylation of p42/p44 MAPK but not AKT and both responses were abrogated in BRMS1-expressing cells. These data indicate that BRMS1 selectively modulates response to mitogenic signals by alteration of cell surface receptors as well as downstream pathways. Thus, 
mechanistically, BRMS1 may suppress metastasis, in part, by differentially interfering with cellular response to extracellular signals and provide an explanation for differential cell growth at orthotopic versus secondary sites. Support: NIH-CA 87728, CA89019, Komen-PDF1122006, National Foundation for Cancer Research.

\section{PD 9-7}

Follistatin, an activin-binding protein, suppresses the production of experimental multiple-organ metastasis by small cell lung cancer cells in NK-cell depleted SCID mice

Hirokazu Ogino ${ }^{1}$, Seiji Yano ${ }^{1}$, Soji Kakiuchi ${ }^{1}$, Hiroaki Muguruma $^{1}$, Kenji Ikuta ${ }^{1}$, Tamir Doljinsuren ${ }^{1}$, Kunihiro Tsuchida ${ }^{2,3}$, Hiromu Sugino ${ }^{3}$, Saburo Sone ${ }^{1}$

${ }^{1}$ Department of Internal Medicine and Molecular Therapeutics, University of Tokushima Gradate School, Tokushima, Japan, ${ }^{2}$ Institute for Comprehensive Medical Science, Fujita Health University, Toyoake-shi, Aichi, Japan, ${ }^{3}$ Institute for Enzyme Research, University of Tokushima, Tokushima, Japan

Multiple-organ metastasis is a critical problem of the management of lung cancer patients. Previously, we established multiple-organ metastasis model of human small cell lung cancer (SCLC) in NK cells-depleted SCID mice, and analyzed gene-expression profiles of metastatic lesions from four different organs (lung, liver, kidney, and bone) using a cDNA microarray. In the process, we identified candidate genes that may affect organ tropism of cancer metastasis. Of these genes, we focused on follistatin (FST), an activin-binding protein, which was up-regulated only in bone metastatic lesion. To determine the role of FST on multiple organ metastasis produced by SCLC cell lines, we transfected a human FST-gene into two SCLC cell lines, SBC-3 and SBC5, and established transfectants secreting a large amount of biologically active FST. FST-gene transfection did not affect the cell-proliferation, motility, invasion, or adhesion to endothelial cells in vitro. Intravenously inoculated SBC-3 or SBC-5 cells into the NK-cell depleted SCID mice produced metastatic colonies into the multiple organs, including the lung, liver, and bone. FST transfectants produced much less number of metastatic colonies in the lung, liver, and bone when compared with their parental cells or vector control clones. Immunohistochemical analyses of liver metastases revealed that the number of proliferating tumor cells and tumor-associated microvessel density were significantly less in the lesions produced by FST transfectants. These results suggest that FST inhibited the multiple-organ metastasis by suppressing angiogenesis through the interaction between tumor cells and organ microenvironments. Further experiments are ongoing to clarify the mechanisms of antimetastatic effect by FST.

\section{PD 9-8}

\section{Impact of sinusoidal architecture and $T$ cell immunity on metastatic tumor cell adhesion in vivo}

Peter Gassmann, Andre Hemping-Bovenkerk, Andreas Enns, Sebastian Kuepper, Joerg Haier

Department of General Surgery, University Hospital Muenster, Muenster, Germany

Introduction The colonization of metastatic target organs by cancer cells requires tumor cell arrest in the microcirculation by specific adhesive interactions. In previous work we demonstrated integrine mediated tumor cell adhesion of human colon carcinoma cells within the hepatic sinusoids of male $\mathrm{CD}$ rats by in vivo fluorescent microscopy. Nevertheless immunologic interference in a xenogenic model and quantification of the rate of mechanical, size restricted cell arrest in the hepatic sinusoids remained elusive. Materials and methods After fluorescence labelling, liver metastatic human HT 29LMM colon carcinoma cells were injected intra-arterially as a single cell suspension in immunocompetent $\mathrm{CD}$ rats ( $250 \mathrm{~g} ; 10^{6}$ cells in $1 \mathrm{ml}$ PBS) or in T cell deficient RNU rats $\left(100 \mathrm{~g}, 0.5 \times 10^{6}\right.$ cells in $0.5 \mathrm{ml}$ PBS$)$. The number of arrested tumor cells was semi-quantitatively analysed as described before and is expressed as cells per 30 microscopic fields (MF). Furthermore the rate of lumen occlusive cells was determined. For some experiments cells were treated with a function blocking anti-beta 4 integrine antibody. The diameter of the hepatic sinusoids was determined by in vivo microscopy. Results The mean sinusoidal lumen diameter in $\mathrm{CD}$ rats was $7.47 \pm 0.39 \mu \mathrm{m}$ and $5.14 \pm 1.11 \mu \mathrm{m}$ in smaller RNU rats $(P<0.05)$. At the end of the $30 \mathrm{~min}$ observation period $32.9 \pm 8.1$ cells $/ 30 \mathrm{MF}$ were adherent in $\mathrm{CD}$ rats $(n=7)$ and the rate of lumen occluding cells was $4 \pm 5 \%$; in RNU rats $(n=6) 26.2 \pm 9.4$ cells $/ 30 \mathrm{MF}$ (n. s.) were adherent and the rate of lumen occluding cells was $20 \pm 12 \%(P<0.05)$. After anti-integrin beta 4 treatment of the cells we found $16.2 \pm 5.5$ cells $/ 30$ MF adherent $(P<0.05, n=7)$ in $\mathrm{CD}$ rats and $12.3 \pm 1.5$ cells $/$ MF adherent cells in RNU rats $(n=6$; $P<0.05)$. Conclusions Early, integrin mediated metastatic tumor cell adhesion in the liver is not influenced by $\mathrm{T}$ cell immunity in a xenogenic animal model. Neverthe- 
less the rate of arrested, lumen occluding tumor cells in smaller RNU rats correlates with the smaller diameter of sinusoidal microvessels compared to $\mathrm{CD}$ rats. The microscopic architecture and morphometric parameters of the liver microvasculature may therefore influence studies of tumor cell adhesion in vivo.

\section{PD 9-9}

\section{Reactive oxygen species (ROS) in the inflammation as an endogenous factor in tumor development and progression}

\section{Futoshi Okada}

Department of Biomolecular Function, Graduate School of Medical Science, Yamagata University, Yamagata, Japan

Tumor progression is the final stage of carcinogenesis and the stage contains acquisition of life-threatening phenotypes such as tumor metastasis. Tumor metastasis is promoted by genetic alterations in each step, and epigenetic changes of crucial genes related to organspecific colonization have been elucidated to some extent. While little is known about the causative factors that convert benign tumors into malignant ones, it has been proposed that metastatic tumor cells originate in a pre-existing or dynamic heterogeneity of tumor cells with metastatic potential that reside in the primary tumor, which expand to clinically distinguishable metastasis in the environment of the host (selection). In the current study, we aimed to identify the endogenous factors that can promote tumor metastasis. Among endogenous factors potentially related to tumor development and progression, reactive oxygen radicals (ROS) are thought to have a major influence. Epidemiological studies have indicated that genotoxic ROS are implicated in one-fourth to one-third of all cancers. To verify the involvement of ROS in tumor development and progression, we utilized mice that have targeted disruption of a gene related to ROS production in inflammatory phagocytes. We herein demonstrate inflammatory-cell-derived ROS as an endogenous factor that can convert benign tumors into malignant ones. QR-32 tumor cells, a clone derived from a murine fibrosarcoma, are non-tumorigenic and non-metastatic when injected into normal C57BL/6 mice. However, the cells are converted into tumorigenic and metastatic tumors once they have grown in vivo in contact with foreign-body (gelatin sponge)-induced inflammatory cells, particularly neutrophils. Intraperitoneal administration of a monoclonal anti-granulocyte antibody, RB6-8C5 (RB6), depleted neutrophils from local inflamed sites in mice with co-implantation of QR-32 tumor cells and gelatin sponge. The arising tumors established from RB6-administered mice showed a significant decrease in metastatic incidence as compared with the tumor cell lines obtained from the mice with administration of control vehicles. We also confirmed the phenomena by using integrin-beta 2 knockout mice that had impaired neutrophil infiltration into inflamed sites. We then investigated whether neutrophil-derived ROS actually stimulate tumor progression, by using the mouse deficient in the gp91phox gene which fails to form the membrane complex of the NADPH oxidase to generate superoxide anion during the phagocyte respiratory burst. Compared to WT mice, gp91 phox $^{-/-}$mice exhibited a marked reduction in acquisition of metastatic phenotype. These results demonstrated that neutrophil-derived ROS is primarily important for benign tumor cells to acquire metastatic phenotype.

\section{PD 10-1}

\section{Identification of the metastatic gene signature of human melanoma: dissection of tumor- and host components}

\author{
Jozsef Timar $^{1}$, Laszlo Puskas ${ }^{2}$, Erzsebet Raso ${ }^{1}$ \\ ${ }^{1}$ National Institute of Oncology, Budapest, Hungary, \\ ${ }^{2}$ Center of Biology, Hungarian Academy of Sciences, \\ Szeged, Hungary
}

With the invent of DNA microarray technology several attempts have been made recently to identify a melanoma-specific gene signature. Comparison of the major studies published in this area indicated that only a dozen of melanoma genes can be collected, the expression of which is repeatedly found in the literature and the majority is confirmed at protein level and functional data are also available. These genes include those of the transcription factor NOTCH2, WNT5A, proliferationassociated genes TOPO2A and $\mathrm{CDC} 2$, membrane receptors FGFR and EphA3, adhesion molecules $\mathrm{N}$ cadherin, b3 integrin and syndecan-4, and the cell surface antigen CD59/protectin, and MIA. We have used three genetically unrelated human melanoma cell lines grown as sc. xenografts in SCID mice to reveal a metastatic melanoma gene signature. To identify relevant genes, we have exploited the manipulation of the host to promote or inhibit spontaneous lung metastasis (new born metastatic versus adult-nonmetastatic mice). With the murine stroma it is possible to separate the melanoma and stromal genes involved in metastatic potential. Expression profiles of primary human melanomas (3 cell lines) were obtained by the $41 \mathrm{k}$ Agilent Whole $\mathrm{Hu}-$ 
man Genome Oligo Microarray. The gene set significantly different in all the three cell lines (min. 2-fold difference) was further validated by qPCR using Taqman card of 96 genes (Applied Biosystem). Using this model, we have identified a 832 metastatic melanoma gene signature $(P-0.05)$, from which we were able to derive a 39 -gene set validated by qPCR. This melanoma metastasis gene signature contained 9 previously reported members including transmembrane heparan sulphate proteoglycan, b3 integrin, AKT and TOPO2A. The largest group of metastasis genes belongs to those involved in the regulation of apoptosis (8) and those involved in developmental regulation (NOTCH1, FOXD3, FZD9, HOXC 13). Expression profiles of the host (mouse) were obtained by the 22k Agilent Mouse Genome Oligo Microarray. Analysis of the mouse stroma permissive for spontaneous metastasis (newborn mice) identified a gene set of 62 significantly upregulated genes $(p-0.05)$ containing 36 known ones. The metastasis promoting stromal gene set was dominated by genes involved in function of peripheral nerves (7) and extracellular matrix remodelling (5). Our data demonstrated that the metastatic melanoma gene set is much larger than the metastasis permissive stromal gene set, suggesting tumor cells as primary players in this interaction. Furthermore, our melanoma metastasis gene signature revealed the AKT pathway, TOPO2A and b3 integrin as feasible molecular therapeutic targets of melanoma progression. Supported by NKFP1a-0024-05.

\section{PD 10-2}

\section{Effects of ceramides on A549 human lung cancer cell growth}

Tianhua Zhang ${ }^{1,2}$, Sumiko Hamanaka ${ }^{1}$, Yui Masuda ${ }^{3,4}$, Kenji Mori ${ }^{5}$, Nobuo Murata ${ }^{2}$, Tatsuo Yamakawa ${ }^{2}$, Shunichiro Kubota ${ }^{1}$

${ }^{1}$ The Department of Life Sciences, Graduate School of Arts and Sciences, The University of Tokyo, ${ }^{2}$ Department of Surgery, Teikyo University Mizonokuchi Hospital, Japan, ${ }^{3}$ Department of Applied Biological Chemistry Graduate School of Agricultural and Life Sciences, The University of Tokyo, ${ }^{4} \mathrm{~T}$. Hasegawa Co., Ltd., ${ }^{5}$ RIKEN, Yokohama Institute, Japan

Ceramides, the central molecule of the sphingomyelin pathway, serve as a second messenger for cellular functions including proliferation, differentiation, growth and apoptosis. In the present study we tested the effects of C2 ceramide (Sigma), and newly synthesized ceramide 7 and 8 of human skin (i.e., Stratum Corneum ceramide) on cell growth or apoptosis of human cultured lung cancer cells (A549). C2 ceramide (20 $\mu \mathrm{M})$ was added in culture media for $24 \mathrm{~h}$. Then, the cell number and viability were analyzed. We found that $\mathrm{C} 2$ ceramide induced cell death. We further investigated signal transduction of $\mathrm{C} 2$ ceramide-induced cell death. Analysis by Western blotting showed that the levels of Bax, Bad and p38 MAP kinase were elevated. To confirm the involvement of p38 MAP kinase in C2 ceramide-induced cell death, the effects of SB 203580 (p38 MAP kinase inhibitor) and U0126 (the MEK inhibitor) on cell death were tested. SB 203580, but not U0126, partially rescued the cell death induced by $\mathrm{C} 2$ ceramide. The result suggests that $\mathrm{p} 38$ MAP kinase was partially involved in $\mathrm{C} 2$ ceramide-induced A549 cell death. We are currently studying the effects of newly synthesized Stratum Corneum ceramide 7 and 8 on cell growth or apoptosis. We will compare the effects of $\mathrm{C} 2$ ceramide and Stratum Corneum ceramide on cell death, and discuss it.

\section{PD 10-3}

\section{Identification and characterisation of novel genetic regulators of breast cancer metastasis}

Bedrich L. Eckhardt ${ }^{1,2}$, Belinda S. Parker ${ }^{1}$, Christina M. Restall ${ }^{1}$, Ryan K. van Laar ${ }^{1}$, Robin L. Anderson ${ }^{1,2}$

${ }^{1}$ Peter MacCallum Cancer Centre, Melbourne, Australia, ${ }^{2}$ University of Melbourne, Melbourne, Australia

The majority of deaths due to breast cancer result from the formation of metastases that arise in distant organs such as lymph nodes, lung and bone. Metastatic spread to these organs leads to debilitating complications, such as respiratory problems, spinal cord compression, bone pain and fractures. Current therapies are palliative, aiming to relieve the symptoms caused by metastatic growth. To achieve curative therapies, a better understanding of the genetics that underlie the process of metastasis is required. To this end, a unique mouse model of spontaneous breast cancer metastasis to multiple sites was characterized both at the phenotypic and genotypic levels. Using a novel realtime quantitative PCR assay of metastatic burden several mouse mammary tumour lines were characterized for their ability to spontaneously metastasise to various tissues. Based on this assay, the tumour lines were categorized as nonmetastatic (67NR), weakly metastatic (4TO 7, 168FARN and 66cl4) and highly metastatic (4T1.2 and 4T1.13). In conjunction, functional assays that mimic the individual events of metastatic progression revealed that highly metastatic tumour lines were more adhesive, migratory and invasive compared to their weaker met- 
astatic counterparts. Each metastatic tumour was array profiled against the non-metastatic $67 \mathrm{NR}$ to elucidate genes associated with metastasis in this model. Gene ontology analysis of the array data revealed that genes involved in adhesion, motility and invasion were inherently altered between weakly and highly metastatic tumours. Further, the genetic differences between highly and weakly metastatic mouse tumours were able to segregate a cohort of human breast cancer patients into "early" and "late" metastasis categories. In highly metastatic tumours, a significant proportion of extracellular matrix (ECM) genes were overexpressed. An ECM gene POEM/nephronectin was identified and evaluated for a functional role in metastasis. Reduction of POEM expression by stable RNA interference in the highly metastatic 4T1.2 tumour line impaired metastasis to lung, bone and kidney. A second gene, bone morphogenic protein-4 (BMP-4), was identified with minimal expression in 4T1.2 tumours. Restoration of BMP-4 expression in 4T1.2 tumours inhibited metastasis and dramatically improved the survival of mice bearing these tumours. These studies implicate a causal role for POEM and BMP-4 in the progression of breast cancer. Further characterisation of these genes will aid in the development of more specific and effective therapies for metastatic breast cancer.

\section{PD 10-4}

\section{The expression of PSCA in human clear cell renal cell carcinoma: a quantitative reverse transcriptase- polymerase chain reaction analysis}

Mohamed M. Gad El-Moula ${ }^{1}$, Tomoharu Fukumori ${ }^{1}$, Essam M. Elsamman ${ }^{2}$, Ryoichi Nakanishi ${ }^{1}$, Ryuichi Taue $^{1}$, Hiroyoshi Nakatsuji ${ }^{1}$, Natsuo Oka ${ }^{1}$, Hirofumi Izaki $^{1}$, Masayuki Takahashi ${ }^{1}$, Kunihisa Yamaguchi ${ }^{1}$, Masa-aki Nishitani ${ }^{1}$, Hiro-omi Kanayama ${ }^{1}$

${ }^{1}$ Urology Department, The University of Tokushima Graduate School of Health Biosciences, ${ }^{2}$ Sohag Cancer Institute-Sohag-Egypt

Objectives To analyze the gene expression level of the prostate stem cell antigen (PSCA) in human clear cell renal cell carcinoma (CCRCC) and its relationship with conventional clinicopathological manifestations. Furthermore, to evaluate its prognostic value for patient outcome and to determine the effect of PSCA on the progression of CCRCC. Patients and methods We quantified PSCA mRNA level in human renal cell carcinoma (RCC) cell lines (ACHN, A704, Caki-1, Caki-2, and KPK-1) and in 154 surgically obtained tissue samples (81 from CCRCC, 73 from normal kidney) using realtime reverse transcriptase-polymerase chain reaction (RT-PCR) and the findings were analyzed in relation to clinicopathological factors. Immunohistochemical expression was examined using confocal laser scanning light -microscopy to validate RT-PCR data. Results PSCA is over-expressed in all RCC cell lines. PSCA mRNA levels increased significantly in CCRCC compared to normal kidney tissue samples $(P<0.0001)$. The PSCA mRNA level was significantly higher in grades $2-3$ than in grade 1 tumors $(P=0.028)$, and in advanced disease (T3-4) than in confined organ disease (T1-2) $(P=0.016)$. Significantly higher PSCA mRNA expression was observed in patients with M1 than in those with M0 $(P=0.029)$. Patients in whom the lesions showed high PSCA expression level had a significantly worse prognosis than those with low PSCA expression level $(P=0.044)$. Immunohistochemical analysis demonstrated that markedly increased PSCA expression in CCRCC compared to normal kidney and in advanced disease high grade compared to confined organ low grade tumors. Conclusions A significant correlation was detected in the gene expression level of PSCA with histological grade, clinicopathological stage and prognosis in CCRCC. Our data indicate that PSCA is associated with carcinogenesis and progression of CCRCC.

\section{PD 10-5}

\section{Mutants of breast cancer metastasis suppressor 1 (BRMS1) reveal its biochemical characteristics in human breast carcinoma cells}

Yi Xie, Alka Mehta, Douglas Hurst, Blake Moore, Ritu Saxena, Danny Welch

University of Alabama-Birmingham, USA

The BRMS1 metastasis suppressor blocks tumorigenicity of breast carcinoma, ovarian carcinoma and melanoma cells when re-expressed. BRMS1 protein localizes to the nucleus and is part of large multimeric complexes containing mSin $3 \mathrm{~A}$ and histone deacetylases. The BRMS1 protein contains glutamate-rich domain (aa 1-48), two coiled-coil/Leucine zipper (LZ) domains (aa 51-81, aa 147-180) and two nuclear localization sequences (NLS1and NLS2, aa 198-205 and 239-245). We previously showed that BRMS1 directly interacts with $\mathrm{Rb}$ binding protein 1 , RBBP1. These interactions and cellular localization presumably affect metastasis by manipulating transcription of downstream mediators. However, the biochemistry of BRMS1 protein interactions and mechanisms of nuclear localization are still 
ill-defined. For this study, we made constructs containing BRMS1 domains as bait and the C-terminus of RBBP1 (aa 1007-1257) as prey in a yeast two-hybrid interaction assay. Mutants of BRMS1 putative nuclear localization sequences were also tested for cellular localization by immunofluorescence microscopy. We found that the second LZ domain is necessary for direct interaction with RBBP1. Specifically, introduction of a mutation (L174D) disrupted RBBP1 specific binding. Replacement of NLS1 by NLS2 (NLS2, 2); replacement of NLS2 by NLS1 (NLS1,1) or switching the order NLS2 and NLS1 (NLS2,1) in green fluorescent protein (GFP) glutathione-S-transferase (GST)-BRMS 1 fusion proteins was done. Chimeras with GFP-GST $\left(M_{\mathrm{r}}\right.$ $78 \mathrm{kDa})$ were too large to freely diffuse into the nucleus, allowing evaluation of the role (s) of each NLS for cellular localization. GFPGST-BRMS1, NLS1,1 and NLS2,1 were predominantly nuclear; whereas, deletion of both NLS and NLS2,2 localized primarily to the cytoplasm. Therefore, NLS1 promotes trafficking of BRMS1 to the nucleus and NLS2 appears to be dispensable. Experiments are underway to determine whether nuclear localization and RBBP1 interaction are essential for the metastasis suppression and transcriptional regulation functions of BRMS1. Support: CA87728, National Foundation for Cancer Research, F32CA113037.

\section{PD 10-6}

\section{Molecular and genetic basis of metastatic progression of mouse colon carcinoma cells}

Yoshimi Ohashi, Nobuaki Higashi, Megumi MorimotoTomita, Tatsuro Irimura

Laboratory of Cancer Biology and Molecular Immunology, Graduate School of Pharmaceutical Sciences, The University of Tokyo

Background and aims We established an experimental mouse model to represent colon carcinoma progression from intermediate to advanced stages by the use of colon 38 cells. Cells were selected for liver metastasis in vivo four times and variant population at each step of the selection were obtained. Present report aims to characterize these variant cells corresponding to the advanced stages in comparison to the parental cells for their in vivo behavior and molecular characteristics. Methods and results The parental colon 38/SL1-SL2 and SL3-SL4 cells corresponding to the advanced stages were examined for the incidence of experimental lung metastasis formation following i.v. injection, incidence of experimental liver metastasis formation following intrasplenic injection, orthotropic growth in the ceca, ectopic growth in the skin, and growth in the liver following intrahepatic injections. SL4 cells were shown to be highly metastatic to the liver and to the lung, whereas colon 38 cells were metastatic only to the liver. These results indicate that SL4 cells, although previously selected for liver metastasis formation, have high metastatic potentials to multiple organs. The growth rates in the soft agar and the size of liver metastasis were significantly higher with SL 3 SL 4 cells than colon 38 , SL1-SL2 cells, and these characteristics correlated to silencing of $C d k n 2 a$ gene expression. The results of PCR amplification of this region and microsatellite markers indicated that genomic deletion but not hypermethylation was the cause of the silencing of $C d k n 2 a$ gene expression. Discussion Gain of capacity for anchorageindependent growth by a loss of $C d k n 2 a$ gene correlated to the increased metastatic potential to multiple organs. This is the first experimental model to show that the phenotype of colon carcinoma progress from organ selective to multiple metastasis.

\section{PD 10-7}

\section{Restored expression of the MYO18B gene suppresses the progression of human malignant pleural mesothelioma cells in SCID mice}

Kenji Ikuta ${ }^{1}$, Seiji Yano ${ }^{1}$, Nobutaka Edakuni ${ }^{4}$, Emiko Nakataki $^{1}$, Hiroaki Muguruma ${ }^{1}$, Hisanori Uehara ${ }^{2}$, Masachika Tani ${ }^{3}$, Jun Yokota ${ }^{3}$, Hisamichi Aizawa ${ }^{4}$, Saburo Sone ${ }^{1}$

${ }^{1}$ Department of Internal Medicine and Molecular Therapeutics, University of Tokushima Graduate School, Tokushima, Japan, ${ }^{2}$ Department of Molecular and Environmental Pathology, University of Tokushima Graduate School, Tokushima, Japan, ${ }^{3}$ Biology Division, National Cancer Center Research Institute, Tokyo, Japan, ${ }^{4}$ First Department of Internal Medicine Kurume University, Kurume, Japan

Background Malignant pleural mesothelioma (MPM) is closely related to exposure to asbestos, and a rapid increase in the number of MPM patients is therefore estimated to occur from 2010 to 2040 in Japan. Since MPM is refractory to conventional chemotherapy and radiotherapy, the prognosis of MPM patients is extremely poor. MYO18B, a novel member of the myosin family, is a tumor suppressor gene isolated from a homozygously deleted region at $22 \mathrm{q} 12.1$ in a lung cancer cell line. The inactivation of the MYO18B gene plays an important role in several malignant diseases. However, the role of MYO18B in the progression of MPM is 
still unknown. Therefore, we investigated whether MYO18B work as tumor suppressor in MPM cells. Methods Six different human MPM cell lines (EHMES1, EHMES-10, MSTO-211H, NCI-H28, NCI-H2052, and NCI-H 2452) were used. MYO18B protein expression was determined by Western blotting. One of the cell lines, EHMES-10, was transfected with the MYO18B gene. The anchorage-dependent or -independent growth was determined by MTT assay and colony formation assay. The motility was determined by wound healing assay. After ectopic (subcutaneous space) or orthotopic (thoracic cavity) cell injection into SCID mice, tumor size or tumor weight and the volume of pleural effusion were measured. We performed immunohistochemical analyses (CD31, VEGF, Ki-67, and TUNEL) of the thoracic tumors produced by the cells. Results Western blot revealed that none of the cell lines expressed a detectable level of MYO18B protein. We found that a restored expression of the MYO18B protein in $\mathrm{EH}-$ MES-10 cells resulted in the inhibition of their anchorage-independent growth and motility in vitro. In addition, it also inhibited their ectopic and orthotopic growth in SCID mice, in association with an increased degree of cell-apoptosis. Furthermore, it also suppressed the production of bloody pleural effusion after orthotopic injection. Conclusion These findings suggest that the restored expression of MYO18B may be a useful therapeutic strategy for the treatment of locally advanced MPM in humans.

\section{PD 10-8}

\section{Gene expression profiling predicts lymph node metastasis in a subpopulation of patients with squamous cervical carcinomas}

Jelmer J. Van Zanden ${ }^{1}$,G. Bea A. Wisman ${ }^{1}$, Alice J. Koning ${ }^{1}$, Haukeline H. Volders ${ }^{1}$, Ed Schuuring ${ }^{2}$, Ate G. J. van der Zee ${ }^{1}$

${ }^{1}$ Department of Gynecological Oncology, University Medical Center University of Groningen, The Netherlands, ${ }^{2}$ Department of Pathology, University Medical Center University of Groningen, The Netherlands

Cancer of the cervix is the second most common cancer in women worldwide. Although cytology based cervical screening markedly improved early detection of cervical neoplasia, a substantial part of early stage cervical cancer patients has nonetheless a poor prognosis due to lymph node metastasis. Gene expression profiling using Affymetrix U133 plus 2.0 arrays on 38 primary tumors of early stage squamous cervical carcinoma patients with complete follow-up (20 pelvic lymph node negative $(\mathrm{N}-)$ vs. 18 lymph node positive $(\mathrm{N}+))$ was performed to identify (i) molecular markers that predict lymph node metastasis, and (ii) to increase understanding of the cell biological processes involved cervical cancer metastasis. Using class comparison and compound covariate prediction analysis we found a minimal set of genes $(n=94$ out of 188 differentially expressed probes at $P<0.001)$ that could correctly classify metastasizing tumors for approximately $70 \%$ of the cervical carcinomas $(P<0.05$ upon permutation). Interestingly, the $30 \%$ misclassified tumors consisted of both $\mathrm{N}-$ and $\mathrm{N}+$ patients. In depth analysis revealed that these patients can hardly be separated into the two groups $(\mathrm{N}-$ vs. $\mathrm{N}+)$ based on their gene expression levels. This observation not only suggests that at least two different mechanisms might be responsible for the metastatic phenotype, but it also implies that classical class-comparison analysis in two relatively small groups, is not an optimal method to cope with a large variance in the data. Therefore, using quantitative real time RT-PCR on a set of 24 selected genes we validated the expression differences between the two groups. This classifier opens the opportunity to study the biological mechanisms responsible for metastasis of at least the majority of patients with early stage cervical cancer. This research is financially supported by The Dutch Cancer Society grant RUG 2004-3161.

\section{PD 10-9}

\section{Signal transduction pathways involved in (-)-epigalllocatechin-3-gallate-induced tumor cell growth suppression}

Yuhi Sakamoto $^{1}$, Lisa Hirose ${ }^{1,2}$, Shunichiro Kubota ${ }^{1}$

${ }^{1}$ Department of Life Sciences, Graduate School of Arts and Sciences, The University of Tokyo, ${ }^{2}$ Graduate School of Human Sciences, Waseda Univ.

(-)-Epigalllocatechin-3-gallate (EGCG), a principal catechin from green tea, is known to modulate multiple signal transduction pathways involved in cell cycle arrest and apoptosis. In the present study we investigated the effects of EGCG on human lung cancer cell (A549) growth in vitro. EGCG inhibited A549 cell growth in a dose-dependent manner. We next studied signal transduction pathway involved in EGCG-induced growth inhibition. EGCG was added in culture media, and after $3,6,12$, and $24 \mathrm{~h}$, cells were harvested. Expression levels of various proteins and phosphorylated proteins involved in MAPK pathway, NF- $\kappa$ B pathway, and cell cycle pathway were analyzed using Western blotting. The phosphorylation levels of ERK1/2, p38, and MEK3/6 
were decreased. The NF- $\kappa$ B level was also decreased. The expression levels of $\mathrm{p} 53$ and $\mathrm{p} 27$ were elevated after $6 \mathrm{~h}$. Over a $12 \mathrm{~h}$, the phosphorylation levels of $\mathrm{Rb}$ was decreased. The data in the present study suggest that EGCG blocks the MAPK pathway, and inhibits NF- $\kappa$ B activation, leading to $\mathrm{G} 1$ arrest by modulating $\mathrm{G} 1$ to $\mathrm{S}$ phase regulatory proteins such as $\mathrm{p} 27, \mathrm{p} 53$ and $\mathrm{pRb}$.

\section{PD 10-10}

\section{Dissecting steps in metastatic colonization regulated by the MKK4 metastasis suppressor protein}

J. Hickson ${ }^{1}$, J. O'Keefe ${ }^{2}$, J. Souris ${ }^{3}$, T. Lotan ${ }^{4}$, D. Vander Griend ${ }^{2}$, S. Yamada ${ }^{1}$, C. Rinker-Schaeffer ${ }^{2}$

${ }^{1}$ Section of Gynecologic Oncology, Department of Obstetrics and Gynecology, The University of Chicago, ${ }^{2}$ Section of Urology, Department of Surgery, The University of Chicago, ${ }^{3}$ Department of Radiology, The University of Chicago, ${ }^{4}$ Department of Pathology, The University of Chicago

Objectives MKK 4 is a MAP $2 \mathrm{~K}$ in the canonical stress-activated kinase cascade. We previously demonstrated a novel metastasis suppressor function for this protein in both prostate and ovarian cancers. Interestingly, our current studies demonstrate that in the SKOV3ip.1 ovarian cancer model MKK4 signals through p38 to suppress metastasis, while in the AT6.1 prostate cancer model MKK4 signals through JNK. In both cases, however, suppression occurred at the step of metastatic colonization. Further, ectopic expression of MKK4 does not increase rates of apoptosis in metastases. We therefore hypothesize that activation of MKK4 culminates in signals that impair the ability of disseminated cells to initiate and/or maintain cell proliferation. Methods Multiple SKOV3ip.1 or AT6.1 clones stably expressing hemagglutin tagged (HA)MKK4, HA-MKK4 (KR) (a kinase inactive form of MKK4), HA-MKK6 (a specific p38 activator), or HAMKK7 (a specific JNK activator) were derived using a pLNCX2 retroviral vector. For the SKOV3 ip. 1 model system, $1 \times 10^{6}$ cells/clone were injected intraperitoneally (IP) into female mice and mean number of metastases compared to vector control clones at the experimental endpoint. For the AT6.1 model, $2 \times 10^{5}$ cells/clone were injected subcutaneously into male mice and the mean number of lung metastases compared to vector control clones. To evaluate the effect of MKK4 kinase activity on animal survival, animals injected with HA-MKK4, HA-MKK4 (KR) or pLNCX2 clones were followed until moribund and mean days of survival compared using both model systems. Apoptotic cells within the lung of AT 6.1-HAMKK4 andpLNCX2 tumor-bearing mice 35 days postinjection were quantified by cleaved caspase $3 \mathrm{immu}-$ nostaining and TUNEL reaction. Results In both models, mice injected with HA-MKK4 (KR) expressing clones had significantly more metastases when compared to mice injected with HA-MKK4 expressing clones, while there was no significant difference compared to vector controls. Furthermore, HA-MKK4 (KR) mice and the control mice survived a significantly shorter time than the HA-MKK4 mice. Interestingly, in the SKOV3ip. 1 model HAMKK 7 expression did not suppress metastasis in vivo, while HA-MKK6 significantly reduced metastases. This is in contrast to the AT6.1 model where HA-MKK7 suppressed metastasis, while HA-MKK6 had no effect. Finally, quantification of apoptotic cells in MKK4- and vector-expressing AT 6.1 metastases revealed no difference between groups. Conclusions MKK4 kinase activity is essential for metastasis suppression and extended survival in both model systems. We observed that in the SKOV3ip.1 model, MKK6 expression suppressed metastatic colonization and MKK 7 had no effect, while the reverse was true in the AT6.1 model. Taken together, these data raise important questions regarding the signaling specificity of MKK4's metastasis suppressor function and how it is regulated in these model systems. Our data indicating no difference in rates of apoptosis between MKK4- and vector-expressing metastases suggest that MKK4 may impair the ability of disseminated cells to initiate and/or maintain cell proliferation. We are currently expanding our methodology to include molecular, biochemical, and imaging techniques to more rigorously test this hypothesis.

\section{PD 10-11}

\section{Network analysis of the signal transduction with metastasis-related factors in human tongue cancer cells}

Masayo Morifuji ${ }^{1}$, Norie Araki ${ }^{2}$, Kousuke Tashiro ${ }^{3}$, Atsushi Yasukochi ${ }^{1}$, Seiji Nakamura ${ }^{1}$

${ }^{1}$ Section of Oral and Maxillofacial Oncology, Division of Maxillofacial Diagnostic and Surgical Sciences, Faculty of Dental Science, Kyushu University, ${ }^{2}$ Graduate School of Medical Sciences, Kumamoto University, Kumamoto, Japan, ${ }^{3}$ Laboratory of Molecular Gene Technics, Department of Genetic Resources Technology

Background It is well known that metastasis is achieved through inter- and intra-cellular signal transduction 
consisted of not only one of individual signal pathways functioning independently, but of a system of signaling networks that are based upon the diverse interactions between proteins. We have integrated $\mathrm{mRNA}$ and protein expression results and have investigated the signaling mechanism of metastasis-related molecules using molecular network search software KeyMolnet. Purpose Through a comprehensive analysis of protein and gene expression, we aim to detect the candidate factors involved in metastasis or metastasis suppression and the corresponding signal transduction mechanisms in tongue cancer cells of different metastatic abilities. Materials and methods iTRAQ was used to identify the proteins and to perform a comparative analysis of their precise quantification in four different specimens simultaneously. Affymetrix DNA-chip was used to systematically analyze gene expression for 54,525 genes. Four types of tongue cancer cell lines (high metastatic ability: SQUU-B, medium metastatic ability: HSC-3, and low metastatic ability: SQUU-A and HSC-4) were analyzed using these systems. KeyMolnet software was then used to perform a comprehensive analysis of the protein and gene expression values. Results Via an LC-MS/MS analysis using iTRAQ, 218 proteins were identified. Comparative analysis of protein expression in cancer cells of high metastatic ability obtained from the same patient revealed 41 proteins with high expression. Nine (9) proteins including cytokeratin 8 , cytokeratin18, T-complex protein 1 subunit epsilon and microphage migration inhibitory factor etc. exhibited high expression for both proteins and mRNA. Sixty (60) proteins with low expression were detected in the same cancer cells. Seven (7) proteins including epidermal fatty-acid binding protein, protein S100-A2, cytokeratin 6B and heat shock protein 10, etc. exhibited low expression for both protein and mRNA. Thirty-six signal transduction pathways including hypoxia-inducible factor signal transduction, were found to be acting on the proteins with high expression, and 33 signal transduction pathways including glucocorticoid receptor-based regulation of expression, were found to be acting on the proteins with low expression in cancer cells of high metastatic ability. Conclusion It is suggested that the comprehensive analysis of protein and mRNA expression in cancer cells of different metastatic abilities may enable the systematic detection of network mechanisms of metastasis-related molecules.

\section{PD 10-12}

\section{Breast Cancer Metastasis Suppressor 1 (BRMS1) is stabilized by the Hsp90 chaperone complex}

Douglas Hurst $^{1}$, Alka Mehta ${ }^{1}$, Blake Moore ${ }^{1}$, Pushkar
Phadke $^{1}$, William Meehan ${ }^{1}$, Mary Ann AccavittiLoper $^{1}$, Lalita Shevde ${ }^{2}$, James Hopper ${ }^{1}$, Danny Welch ${ }^{1}$, Rajeev Samant ${ }^{2}$

${ }^{1}$ University of Alabama-Birmingham, USA, ${ }^{2}$ University of South Alabama, USA

Breast Cancer Metastasis Suppressor 1 (BRMS1), a recently discovered metastasis suppressor, suppresses metastasis without blocking tumorigenesis in multiple human and murine cancer cells. BRMS1 is part of multi-protein complexes ( $>1 \mathrm{MDa}$ total mass) that include $\mathrm{mSin} 3$ and HDAC-1/2 which function in transcriptional regulation. However, the complete set of proteins involved in these complexes are incompletely known. Yeast two-hybrid experiments identified eight genetic interactors of BRMS1; six of these are novel BRMS1 interacting proteins (BIPs) that are not part of the core mSin 3 complex. One of these BIPs, Hsp40, is part of the Hsp90 chaperone complex that includes Hsp70 and these chaperones were identified as BIPs using immuno-affinity chromatography combined with MALDI-TOF-MS. Co-immunoprecipitation experiments validated proteins as BIPs. To characterize the functional interaction of the chaperone BIPs with BRMS1, cells were treated with the Hsp90 inhibitor geldanamycin (GA). BRMS1 was degraded in a dosedependent manner in GA treated cells that was rescued by treatment with the proteasome inhibitor MG-132. Hsp90 client protein stabilization has been recently shown to be regulated by HDAC- 6 . Co-immunoprecipitation experiments identified HDAC-6 and two other class II HDACs, HDAC- 4 and -5 as BIPs. These data demonstrate that BRMS1 binds to multiple protein complexes in addition to the core $\mathrm{mSin} 3$ complex and is dynamically regulated by the Hsp90 chaperone complex which may be important in maintaining the functional role of BRMS1 in metastasis suppression. Support: CA87728 (DRW), F32CA113037 (DRH), CA89019 (DRW), National Foundation for Cancer Research (DRW), BCTR 0503488 (RSS) and BCTR0402137 (LRS).

\section{PD 10-13}

\section{A-to-I RNA editing of a homeobox gene in a subset of human cancer cells}

Meiko Takahashi ${ }^{1}$, Takanobu Yoshimoto ${ }^{1,2}$, Hajime Kubo $^{1}$

${ }^{1}$ Molecular \& Cancer Research Unit, HMRO, Graduate School of Medicine, Kyoto University, Japan, ${ }^{2}$ Department of Gastroenterology \& Hepatology, 
Graduate School of Medicine, Kyoto University, Japan

RNA editing is a post-transcriptional process that modifies genetic information at the transcriptional level. It seems to be widespread in human nervous system, but until now the evidence of RNA editing as a potential oncogenic principle has only come from limited tumor types. Recent several computational analyses for human ESTs and genome databases have identified over a thousand new editing substrates, although much of the cellular machinery involved in cancerous growth are still unknown. Here we show that a homeobox gene loses its tumor suppressing activity through RNA editing and not DNA mutations. From Analyses of a subset of human pancreatic and colon cancer cell lines, we identified four common editing sites in its coding region. A to $G$ base change was found in cDNA taken from the cancer cells but not in the corresponding genomic DNA, nor in normal human cDNA libraries of all cell types studied. This homeobox gene has been reported to be involved in cellular proliferation during embryogenesis. These findings may point to a pivotal role for A-to-I RNA editing in the pathogenesis of human cancer progression. Since the genome is intact, RNA mutations may be repaired if the mechanisms underlying RNA editing are identified, and our report would greatly contribute to provide new drugs and novel therapies for cancer patients.

\section{PD 11-1}

\section{Combination effect of transforming growth factor \& beta-receptor inhibitor and S-1 on the lymph node metastasis for gastric cancer}

Hidemi Kawajiri, Masakazu Yashiro, Tendo

Masashige, Nobuya Yamada, Tetsuji Sawada, Kosei

Hirakawa

Department of Surgical Oncology, Osaka City University Graduate School of Medicine, Osaka, Japan

Human scirrhous gastric cancer is characterized by cancer cell infiltration and proliferation accompanied by extensive stromal fibrosis. It has been reported that transforming growth factor \& beta-receptor (TGF \& beta-R) is expressed intensely in scirrhous gastric cancer cell, TGF \& beta is produced from both scirrhous gastric cancer cell and fibroblast, and both were associated to invasion of scirrhous gastric cancer. TGF \& beta-R inhibitor "A-77" obstructs phosphorylation of Smad 2 of an ATP binding site of TGF \& beta-R. In this study, we examined the effect of A-77 for scirrhous gastric cancer in vivo. Materials and method Scirrhous gastric cancer cells "OCUM-2MLN" was inoculated to orthotopically at greater curvature of the antrum in nude mice and developed orthotopic implantation. Seven days after, A77 was administered intraperitoneally 3 times per week and/or S-1 was administered orally 5 times per week. More 4 weeks after, they were sacrificed. We divided these into control group, A-77 treated group, S-1 treated group, or A-77 and S-1 combination group. We examined the effect of TGF \& beta-R inhibitor with or without an anticancer agent on tumor size, lymph nodes metastasis and histological findings. Result Tumor size was smaller in an A-77 treated group, a S-1 treated group, A-77 and S-1 group than control group. The lymph node metastasis was significantly restrained by A-77 and S-1. Fibrosis was reduced by A-77 and $\mathrm{S}-1$ combination histologically. Conclusion TGF \& beta- $\mathrm{R}$ inhibitor combined with anticancer agent is useful for the treatment of scirrhous gastric cancer.

\section{PD 11-2}

\section{$\mathrm{NF}-\kappa \mathrm{B}$ decoy inhalation as a treatment for osteosarcoma metastasis to lung}

Tetsuho Fujimoto, Shigeki Kakunaga, Yuki Kishida, Tetsuya Tomita, Akira Myoui, Takafumi Ueda, Hidaki Yoshikawa

The Department of Orthopaedics, Osaka University Graduate School of Medicine, Osaka, Japan

Nuclear factor $-\kappa \mathrm{B}(\mathrm{NF}-\kappa \mathrm{B})$ is a transcription factor that has crucial roles in inflammation, cell proliferation and apoptosis. A deregulation of NF- $\kappa \mathrm{B}$, e.g. constitutively activated NF- $\kappa \mathrm{B}$ signaling pathways is one of the important characteristics of cancer including osteosarcoma. We previously showed that NF- $\kappa \mathrm{B}$ is highly activated in murine osteosarcoma cell line, LM8 and an inhibitor of NF- $\kappa \mathrm{B}$ effectively prevented the development of metastatic foci in spontaneous lung metastasis model by inoculating LM8 subcutaneously. In this study, we tested whether inhalation of double-stranded oligodeoxynucleotides (ODNs) of NF- $\kappa \mathrm{B}$ binding site inhibit the development of lung metastasis by binding to and by inhibiting the activity of NF- $\kappa \mathrm{B}$ as a decoy in spontaneous lung metastasis model using LM8 osteosarcoma cells. Transfection of NF- $\kappa \mathrm{B}$ decoy ODNs inhibited the LM8 cell growth estimated by WST-1 assay in comparison with scramble decoy ODNs that lack $\mathrm{NF}-\kappa \mathrm{B}$ specific binding affinity. Before testing the effect of decoy ODNs inhalation on lung metastasis, we determined the distribution of inhaled liquid by administering black dye solution through respiratory tract from nose. The dye distribution was mainly 
confirmed in lung tissue adjacent to hila. Next, LM8 cells $\left(1 \times 10^{5}\right)$ were inoculated subcutaneously to $\mathrm{C} 3 \mathrm{H} /$ He mice and $1000 \mu \mathrm{g}$ of NF- $\kappa \mathrm{B}$ decoy ODNs dissolved in $25 \mu \mathrm{l}$ phosphate buffered saline was instilled into mice nostrils under anesthesia everyday. After four weeks, mice were sacrificed and subcutaneous tumors and lungs were collected for histological examination. We also evaluated the effect of NF- $\kappa \mathrm{B}$ decoy ODNs inhalation on the survival of mice bearing LM8 cells. Histological examination of the lung tissue revealed that lung metastasis was reduced in NF- $\kappa \mathrm{B}$ decoy treated mice compared to that in untreated control mice $(P=0.026)$. In addition, survival rate of the NF- $\kappa \mathrm{B}$ decoy treated group was significantly better than that in untreated group $(P=0.001)$. These results suggest that the inhalation of NF- $\kappa \mathrm{B}$ decoy ODNs could be a new therapeutic strategy for osteosarcoma metastases to lung. Because decoy ODNs are easy to be degraded in blood serum, its direct administration through respiratory tract could give a useful solution for its use for the treatment of lung metastasis.

\section{PD 11-3}

\section{The effect of parthenolide on osteosarcoma cell colonization in lung in tail-vein injection model}

\author{
Yuki Kishida ${ }^{1,2}$, Hideki Yoshikawa ${ }^{2}$, Akira Myoui ${ }^{2,3}$ \\ ${ }^{1}$ Department of Kampo Medicine, Osaka University \\ Graduate School of Medicine, Osaka, Japan, ${ }^{2}$ Depart- \\ ment of Orthopaedics, Osaka University Graduate \\ School of Medicine, Osaka, Japan, ${ }^{3}$ Department of \\ Medical Center for Translational Research, Osaka \\ University Hospital, Osaka, Japan
}

The nuclear factor $\kappa \mathrm{B}(\mathrm{NF} \kappa \mathrm{B})$ is a ubiquitous transcription factor known to regulate the transcription of many genes including genes associated with anti-apoptosis, cell cycle regulation, motility and neovascuralization. LM8 cell line is a highly metastatic subclone of Dunn murine steosarcoma. LM8 exhibited constitutively high $\mathrm{NF} \kappa \mathrm{B}$ activity compared to Dunn osteosarcoma with low metastatic potential. It has been reported that suppressing the activation of $\mathrm{NF} \kappa \mathrm{B}$ in highly metastatic tumor cells results in the reduction of metastasis; however, little has been known about the role of $\mathrm{NF} \kappa \mathrm{B}$ in metastasis. Parthenolide, a sesquiterpene lactone, was reported to inhibit the DNA binding of $\mathrm{NF} \kappa \mathrm{B}$. We previously found that parthenolide inhibited lung metastasis but not primary tumor growth in spontaneous lung metastasis model by inoculating LM8 cells subcutaneously. We also found that the genetic inhibition of $\mathrm{NF} \kappa \mathrm{B}$ activity by expressing mutant $\mathrm{I} \kappa \mathrm{B} \alpha$ suppressed lung metastasis in vivo but not subcutaneous tumor growth. Thus, to clarify where parthenolide works during the multistep metastatic process, we examined the effect of parthenolide on metastasis-associated phenotypes in vitro and the effect in a lung metastasis model by tail vein injection. We found that parthenolide strongly induced apoptosis and inhibited cell proliferation and the expression of vascular endothelial growth factor in vitro. Our in vivo experiments by intravenous inoculation showed that lung metastasis was suppressed when parthenolide was administrated starting concurrently with intravenous administration of tumor cells. In contrast, lung metastasis was not reduced when parthenolide was administrated after homing of the tumor cells. Then, we examined the effect of parthenolide on the homing of tumor cells to lung tissue. In an intravenous inoculation model using fluorescence-labeled LM8 cells, parthenolide treatment did not affect the tumor cell arrest in lung vasculature at $4 \mathrm{~h}$ after cell inoculation. However, fluorescence (derived from tumor cells) in lung tissue gradually decreased over time with parthenolide treatment with a significant difference at $48 \mathrm{~h}$, suggesting that parthenolide, at least in part, induced tumor cell death before the cells can form (multicellular) micrometastatic foci. In addition, inhibiting $\mathrm{NF} \kappa \mathrm{B}$ activity by overexpressing $\mathrm{I} \kappa \mathrm{BM}$ suppressed lung metastasis in tailvein injection model. This supports our notion that the metastasis-preventing effect of parthenolide is mediated at least in part by inhibition of $\mathrm{NF} \kappa \mathrm{B}$ activity of tumor cells. These findings suggested that $\mathrm{NF} \kappa \mathrm{B}$ is a potential molecular target for designing specific prophylactic interventions against distant metastasis and that parthenolide is a hopeful candidate for an anti-metastasis drug.

\section{PD 11-4}

\section{The disintegrin eristostatin causes changes in serine- threonine phosphorylation in fibronectin-adherent melanoma cells}

MaryAnn McLane, Stephanie DiRosato, Carrie Paquette-Straub

${ }^{1}$ Department of Medical Technology, University of Delaware, Newark, Delaware

The 49-amino acid disintegrin eristostatin, derived from the viper Eristicophis macmahoni, can, at $1 \mu \mathrm{M}$ concentration, significantly inhibit lung colonization by three human malignant melanoma cell lines in a hematogenous murine model. The mechanism used by this natural protein is not likely to be through inhibition of 
proliferation since it has no effect on cell number in tissue culture or soft agar assays. Inhibition of angiogenesis is also not a mechanism since the integrins most often involved in that process, $\alpha \mathrm{v} \beta 3$ and $\alpha 5 \beta 1$, are not recognized by eristostatin. This disintegrin does, however, prevent cell motility on fibronectin in an in vitro scratch assay. To examine the possibility that this may be through a serine/threonine protein phosphorylation pathway, we analyzed whole cell lysates of melanoma cells grown on fibronectin in serum-starved conditions, treated with buffer or eristostatin for varying time points. While eristostatin influenced both serine and threonine protein phosphorylation in all the cell lines used (vertical growth phase WM 164, highly metastatic C8161, M24 met, 1205LU, MV3), there was no single commonly affected protein found. In addition, several motility-related proteins did show change with eristostatin treatment. Interestingly, one protein $(\sim 17 \mathrm{kDa})$ showed a 2- to 24-fold decrease in serine phosphorylation over the time course in M24met, 1205LU and WM 164 , while another $(\sim 20 \mathrm{kDa})$ was decreased 4 - to 11fold over time in MV3, M24 met and 1205LU. It is possible that the phosphorylation effect induced by eristostatin is more on proteins phosphorylated through tyrosine than serine or threonine, and concurrent studies using anti-phosphotyrosine are being performed to evaluate those signaling pathways related to motility.

\section{PD 11-5}

\section{Oleamide derivatives potently inhibit the spontaneous metastasis of mouse melanoma BL6 cells}

\author{
Yukiko Kanao $^{1}$, Yusuke Ohba ${ }^{1}$, Yasuyuki Kita ${ }^{1}$, \\ Hiroshi Nojima ${ }^{2}$
}

${ }^{1}$ Department of Synthetic Organic Chemistry, Graduate School of Pharmaceutical Science, Osaka University, Osaka, Japan, ${ }^{2}$ Department of Molecular Genetics, Research Institute for Microbial Diseases, Osaka University, Osaka, Japan

We previously reported that overexpression of connexin 26 (Cx26) enhances the spontaneous metastasis of mouse BL6 melanoma cells [1], and that daily intraperitoneal injections of oleamide inhibits the spontaneous metastasis of BL 6 cells [2]. In the present study, we chemically synthesized oleamide derivatives to obtain effective inhibitors of spontaneous metastasis via specific inhibition of Cx26. We chemically synthesized dimeric, trimeric and tetrameric forms of oleamide derivatives and tested for their ability to inhibit the gap junction-mediated intercellular communications (GJIC) that are formed between HeLa cells by the ectopic expression of Cx26. Since inhibition of $\mathrm{Cx} 43$ might cause sudden death due to dysfunction of cardiac Cx43, we synthesized oleamide derivatives that might inhibit Cx26 but not Cx43. Two of these, MI-18 (Metastasis Inhibitor-18) and MI-22 inhibited the Cx26 mediated GJIC as well as oleamide but not $\mathrm{Cx} 43$ mediated GJIC at all. Moreover, their daily injections potently inhibit the spontaneous metastasis of BL6 cells down to about $15 \%$ of that in the untreated control mice. They were safe because even after $>7$ weeks of daily injections, the survival rate of the mice was more than $90 \%$. They also inhibited the rapid tumor growth of AZ521 human gastric cancer cells that were implanted in nude mice. Notably, no cytotoxic effect on AZ521 cells was observed when they were added to the tissue culture medium. The results suggests that MI-18 and MI-22 block the spontaneous metastasis by inhibiting the growth of small metastasized tumor cells. Thus, we propose that oleamide derivatives may serve as prototypes for novel and clinically important anti-cancer drugs.

\section{References}

1. Ito et al (2000) JCI

2. Ito et al (2004) Carcinogenesis

\section{PD 11-6}

\section{Effects of the green tea catechin, EGCG, upon breast cancer growth and osteoclast differentiation}

John T. Price ${ }^{1}$, Jessica Vieusseux ${ }^{1}$, Michelle Kouspou ${ }^{1,3}$, Kelly Waldeck ${ }^{1,2,3}$, Joseline Ojaimi ${ }^{1}$, Julian Quinn ${ }^{2}$

${ }^{1}$ Cancer Biology and Metastasis Laboratory, Department of Biochemistry and Molecular Biology, Monash University, Melbourne, Australia, ${ }^{2}$ StVincent's Institute, Melbourne, Australia, ${ }^{3}$ Department of Medicine, University of Melbourne, Melbourne, Australia

Metastatic dissemination of cancer, rather than the growth of the primary tumour, is ultimately responsible for treatment failure, morbidity and death amongst cancer patients. In breast cancer, bone metastasis is frequent and results in osteolysis of the bone leading to pain, hypercalcemia and pathological fractures. Currently few treatment options are available and no preventative measures are available. To identify potentially effective agents in the treatment of bone metastasis, we have investigated compounds that would not only be effective in abrogating breast cancer cell growth but are equally 
effective at inhibiting the generation of osteoclasts, the major host cell type responsible for bone resorption. Through these investigations we have identified the major green tea catechin, epigallocatechin gallate (EGCG), as a potentially effective agent in the treatment of breast cancer metastasis to the bone. We have determined that EGCG is effective at inhibiting adhesion-dependent andindependent growth in both estrogen-dependent (BT474, MCF-7) and estrogen-independent (MDA-MB-231, MDA-MB-435) breast cancer cells. Examination of the pro-apoptotic enzyme, caspase-3, demonstrated that treatment of MDA-MB-231 and MDA-MB-435 cells with EGCG resulted in increased caspase- 3 activation. In addition to effects upon cell growth, EGCG was also found to inhibit breast cancer cell chemotactic migration towards EGF and IGF-I. As well as effects upon breast cancer cells, EGCG was also found to potently inhibit the differentiation of macrophages to mature osteoclasts in a number of in vitro osteoclast generation assays. These results indicate that EGCG may be effective in the treatment of breast-bone metastasis through its ability to not only target the breast cancer cell but also to perturb the host cells of the bone microenvironment which support and aid in the generation and pathology of bone metastasis.

\section{PD 11-7}

\section{3-Methyladenine suppresses the migration and invasion of HT1080 fibrosarcoma cells independently of autophagy inhibition}

Shingo Ito, Nobuko Koshikawa, Shigenobu Mochizuki, Keizo Takenaga

Division of Chemotherapy, Chiba Cancer Center Research Institute, Chiba, Japan

Aim 3-Methyladenine (3-MA), a well-known inhibitor of autophagic sequestration, has been demonstrated to inhibit class I and class III phosphoinositide 3 (PI3)kinases. Other PI3K inhibitors, wortmannin and LY294002, have been reported to suppress cell migration of tumor cells and autophagy as well. In this study, we examined whether 3-MA suppresses migration and invasion of tumor cells and, if so, whether inhibition of autophagy is, at least in part, involved in the suppression of cell migration by 3-MA. Methods Highly metastatic human fibrosarcoma HT1080 cells were used in this study. Cell migration and invasion assays were performed using FluoroBlok Transwell inserts attached with uncoated and Matrigel-coated filters, respectively. Fetal bovine serum was used as a chemoattractant. F-actin was visualized by staining the cells with rhodamine-phalloidine.
Autophagic vacuoles were detected by staining the cells with monodansylcadaverine. Assay of PI3K activity was performed on the immunoprecipitates using phosphatidylinositol as a substrate. Beclin 1 small interfering RNA (siRNA) was transfected with Lipofectamine 2000. Results Treatment of HT1080 cells with 3-MA $(10 \mathrm{mM})$ resulted in the suppression of actin rich lamellipodia formation. Accordingly, the migration of the cells was markedly suppressed. 3MA showed no cytotoxicity during the migration assays. Both wortmannin $(100 \mathrm{nM})$ and LY $294002(25 \mu \mathrm{M})$ suppressed lamellipodia formation and migration of HT1080 cells, but the effects were weaker than those of 3-MA while wortmannin and 3-MA inhibited class I and III PI3Ks to a similar extent. HT1080 cells showed a significant number of autophagic vacuoles under normal culture conditions. Down-regulation of Beclin 1, a protein required for autophagic body formation, by transfection of Beclin 1-specific siRNA suppressed the autophagy but not the migration of HT1080 cells. Importantly, 3-MA strongly suppressed the invasiveness of HT1080 cells compared to wortmannin and LY 294002. Conclusion The results suggest that 3-MA suppressed the migration and invasion of HT1080 cells independently of autophagy inhibition. The data also suggest that there may exist other 3-MA target molecules that are involved in regulating cell motility than wortmannin/LY 294002-sensitive kinases.

\section{PD 11-8}

\section{TGF-b1 in regulation of glutathione and drug sensitivity of normal and metastatic cells}

\section{Megan C. Eaton, Qiao Zheng, Andrei Bakin}

Department of Cancer Genetics, Roswell Park Cancer Institute, Buffalo, NY, USA

Cancer treatment failure and patient relapse are associated with resistance to anticancer therapies and the development of distant metastasis. Traditionally these two areas have been researched independently however, recent studies have pointed to a connection between drug resistance in tumor cells and the metastatic potential. It has been observed that some tumor cells selected for resistance to drugs are more invasive/ metastatic relative to nonresistant parental cell lines. Other studies have reported that secondary (more metastatic) tumors are more resistant to chemotherapy than their primary counterparts. There is also evidence that metastases growing at different sites can exhibit different sensitivity to drugs. Understanding the mechanism of drug resistance of metastatic cells is important for developing effective therapeutics. 
Transforming growth factor beta 1 (TGF-b1) downregulates a class of genes encoding phase II detoxification proteins via the transcription repressor, activating transcription factor 3 (ATF3). A subset of these genes is responsible for glutathione-dependent metabolism of drugs and intracellular redox balance. Glutathione is vital to antioxidant defense, xenobiotic metabolism, immune modulation, and regulation of cell death and proliferation. Although glutathione is critical for detoxification of carcinogens, elevated levels of glutathione in cancer cells contribute to multi-drug resistance. Here we investigated regulation of phase II genes and levels of glutathione in metastatic breast cancer MDA-MB-231 cells that exhibit TGF-b1 responses but are not growth inhibited by TGFb1. These cells are highly resistant to DNA damaging drugs such as cisplatin. We found that in MDA-MB-231 TGF-b1 failed to increase ATF3 proteins levels and downregulate the phase II detoxification protein, gamma-glutamylcysteine synthetase catalytic subunit (GCLC), the rate-limiting enzyme in glutathione biosynthesis. To address the role of ATF 3, we have created an inducible system in which ATF3 is expressed under control of the tet operon in MDA-MB-231 and normal mammary gland cells (NMuMG). Over-expression of ATF3 reduced proliferation of both cell lines and reduced GCLC protein level. This system will be utilized for investigation of sensitivity of MDA-MB-231 cells to DNA damaging agents. We have developed small interfering RNA to suppress expression of ATF3 in NMuMG cells. This is expected to increase glutathione levels and mimic the drug resistant phenotype observed in MDAMB-231. We will determine if ATF3 affects drug resistance and metastatic potential of MDA-MB-231 cells. The progress of these studies will be presented at the meeting.

\section{PD 11-9}

\section{A new anticancer activity of Iressa (ZD1839) through induction of BRAK/ CXCL14 gene expression}

Shigeyuki Ozawa ${ }^{1,4}$, Yasumasa Kato ${ }^{2,4}$,Yojiro

Maehata $^{2,4}$, Eiro Kubota Kubota ${ }^{1,4}$, Yukari ImagawaIshiguro $^{3}$, Takahide Taguchi ${ }^{3}$, Mamoru Tsukuda ${ }^{3}$,

Ryu-Ichiro Hata ${ }^{2,4}$

${ }^{1}$ Department of Oral and Craniofacial Surgery, Kanagawa Dental College, Kanagawa, Japan, ${ }^{2}$ Department of Biochemistry and Molecular Biology, Kanagawa Dental College, Kanagawa, Japan, ${ }^{3}$ Department of Biology and Function in the Head and Neck, Yokohama City University School of Medicine, Kanagawa, Japan, ${ }^{4}$ Department of Oral Health Science
Research Center, Kanagawa Dental College, Kanagawa, Japan

Objective BRAK, also known as CXCL 14, is a non-ELR motif chemokine, It plays a role not only in an immune recognition processes but also as an antiangiogenic factor. It is reported that mRNA for BRAK is not detected in head and neck squamous cell carcinoma (HNSCC). We recently found that HSC-3 tongue carcinoma cells expressed significant amount of BRAK mRNA and the expression was specifically down regulated by the treatment with epidermal growth factor (EGF). Thus we investigated whether ZD1839, a specific inhibitor for EGF receptor tyrosine kinase, restored the BRAK mRNA expression, and whether this restoration was involved in anticancer activity of ZD1839. Materials and method BRAK expression vector was constructed from the mRNA of HSC- 3 cells by TA cloning strategy, and introduced into HSC-3 cells. High BRAK expressing clones were selected by G418 treatment. Three highBRAK expressing clones were pooled (HSC-3/BRAK) and used for in vivo experiments. Results It has been reported that mRNA for BRAK is barely detected in HNSCC, thus we measured BRAK mRNA levels by RTPCR in 19 kinds of HNSCC cell lines and found that 7 cell lines expressed significant amount of BRAK mRNA and this frequency was much higher than that of previous reports. In addition, EGF down-regulated mRNA levels of BRAK in all of these cell lines. Furthermore, ZD1839 treatment restored or even increased the levels of BRAK mRNA in all the 7 cell lines including HSC-3 cells. In order to find relationship between BRAK expression and tumor suppression, we injected BRAK-expressing HSC3/BRAK cells into back skin of nude mice and found that significantly higher percentage of xenografts were suppressed tumor formation. In addition, the size of tumors formed were smaller than those of Mock-Vector transfected control cells. Oral administration of ZD1839 into nude mice carrying tumors formed by HSC-3 cells increased mRNA levels for BRAK as well as reduction of the size of the tumors. Interestingly, neither anticancer activity, nor induction of BRAK mRNA expression was not found by oral administration of ZD1839 in tumors formed by $\mathrm{KB}$ cells that did not express mRNA for BRAK at all. Conclusion Our results indicated inverse relationship between BRAK mRNA expression and the size of tumors and suggest that ZD1839 express antitumor activity by increasing expression of BRAK/CXCL14.

\section{PD 11-10}

\section{Disulfide bond formation is required for the activation of heparanase}


Siro Simizu ${ }^{1}$, Makoto Muroi ${ }^{1}$,Satoshi Takagi ${ }^{1,2}$, Ngit Shin Lai ${ }^{1,2}$,Hiroyuki Osada ${ }^{1,2}$

${ }^{1}$ Antibiotics Laboratory, DRI, RIKEN, Saitama, Japan, ${ }^{2}$ Graduate School of Science and Engineering, Saitama University, Saitama, Japan

Heparan sulfate (HS) and heparan sulfate proteoglycans (HSPGs) that are located in the extracellular matrix (ECM) and on the external surface of cell membranes play a major role in cell-cell and cellextracellular interactions. Since HS chains tightly bind to a diverse repertoire of proteins under physiological conditions, the enzymatic cleavage of HS by heparanase is likely to be involved in several biological phenomena, including cancer metastasis and angiogenesis. Human heparanase cDNA contains 543 amino acids with six $N$ glycosylation sites and five Cys residues. Overexpression of heparanase has been observed in many human tumor types, such as those in the head and neck; such associations are thought to indicate the involvement of heparanase in tumor progression. Although we previously demonstrated that $N$-glycosylation was required for the secretion of heparanase protein in cultured cells, other post-translational modifications responsible for the regulation of heparanase function have yet to be elucidated. In order to develop heparanase inhibitors, it will be necessary to understand the mechanisms of activation of heparanase in human tumor cells.

In this study, we demonstrate that two disulfide bonds, Cys127-Cys179 and Cys437-Cys542, are formed in heparanase revealed by MALDI-TOF mass spectrometry and biotin maleimide-binding assay. Although disulfide bond formation between Cys437 and Cys542 is necessary for the secretion and activation of heparanase, Cys127-Cys 179 linkage exerts only a negligible effect on secretion. Reducing reagents, such as DTT and 2-mercaptoethanol did not inhibit the heparanase activity in vitro, suggesting that once heparanase is secreted and activated, disulfide bond formation itself is no longer required for enzymatic activity. Alignment of the human amino acid sequence with that of the rat, mouse, cow, dog, chicken, zebrafish, thale-cress, rice plant, and silkworm has shown that the Cys437 and Cys542 residues of human heparanase are well conserved among all forms; however, other Cys residues (namely, Cys127, Cys-179, and Cys211) are not conserved in plants and worms. Thus, it is suggested that the disulfide bond between residues Cys437 and Cys542 in heparanase may be a common and pivotal linkage determinative of the secretion and activation of this enzyme in various organisms.

\section{PD 11-11}

eMIP, a derivative of MIP- $1 \alpha$ enhances the efficacy of radiation and induces an abscopal effect in cancer

Keiichi Nakagawa ${ }^{1}$, Kenshiro Shiraishi ${ }^{1}$, Yoshiro Ishiwata $^{2,3}$, Kouji Matsushima ${ }^{3}$, Takuya Tamatani ${ }^{2}$, Shiro Kanegasaki ${ }^{2}$

${ }^{1}$ Department of Radiology, University of Tokyo, Tokyo, Japan, ${ }^{2}$ Effector Cell Institute, Inc., ${ }^{3}$ Department of Molecular Preventive Medicine, University of Tokyo, Tokyo, Japan

Background To enhance the efficacy of local tumor irradiation in radiotherapy of cancer patients, it is important to induce inflammation and to recruit tumor specific $\mathrm{T}$ cells and dendritic cells (DC) at cancer sites. Macrophage inflammatory protein $1 \alpha$ (MIP- $1 \alpha$, CCL3) is a chemoattractant for DCs, monocytes and lymphocytes, and have a crucial role in development of inflammation and immune response. Ionizing radiationinduced reduction of tumor growth outside the field of radiation, known as the abscopal effect is important for tumor control and is mediated through immune system. We studied the effect of MIP- $1 \alpha$ derivative, eMIP (formerly called BB10010) on irradiated subcutaneous tumor and the growth of tumor implanted in nonirradiated site in mice. Methods C57 BL/6 mice were subcutaneously implanted with Lewis lung carcinoma cells in the right flank. When the diameter of solid tumor reached approximately $1 \mathrm{~cm}$, local tumor irradiation with $6 \mathrm{~Gy}$ was applied. After $20 \mathrm{~h}$, the mice were received eMIP intravenously. Since then, eMIP was administrated once a week. The effect of irradiation and eMIP was evaluated by measuring the tumor volume. In the study of an abscopal effect, mice were subcutaneously implanted with 3LL in the right and left flank and given irradiation only in the right side to investigate the effect outside the irradiated field. Results Radiation with 6 Gy reduced the tumor volume by $50 \%$, and administration of eMIP enhanced the efficacy of radiation by further $30 \%$ (total reduction rate: $80 \%$ ). The optimal dose of eMIP was $2 \mu \mathrm{g} /$ mouse, and eMIP did not have anti-tumor activity by itself in this system. eMIP inhibited the growth of irradiated and non-irradiated 3LL tumor in the right and left flank of mice in a dose dependent manner. Interestingly, this abscopal effect induced by eMIP was not tumor-specific. The similar results were obtained in the study using $\mathrm{BALB} / \mathrm{c}$ mice implanted with Colon26 cells. The tumor treated with both radiation and eMIP showed marked infiltration 
of CD4 and a slight increase of CD8 positive cells compared with control and radiation-treated group. Infiltration of CD4 and CD8 positive cells was also increased in the non-irradiated tumor tissue as well as in the irradiated tumor tissue in radiation/eMIP treatment. Conclusion The results indicate that eMIP enhances the radiation-induced inflammatory response and/or tumor specific immune reaction in tumor, and prevention of the metastasis via the abscopal effect. This might be a new approach in the radiation therapy.

\section{PD 11-12}

\section{A recombinant human RNASET2 glycoprotein with anticarcinogenic and antiangiogenic characteristics: induction, purification and characterization}

\section{B. Schwartz, P. Smirnoff, L. Roiz, B. Angelkovitch, O. Shoseyov}

Hebrew University of Jerusalem, Rehovot Israel

Human RNASET2 is a T2-RNase glycoprotein encoded by the RNASET2 gene which is located on chromosome 6 (6q27). Deletion in 6q27 is associated with several human malignancies. A synthetic RNASET2 gene optimized for expression in the yeast Pichia pastoris was designed according to the cDNA sequence and cloned under the control of an expression vector. The recombinant protein was purified through an affinity Sepharose-ConA column. Actin-binding activity was demonstrated by membrane blotting followed by antiactin immunolabeling and by crosslinking in solution to G-actin using 1-[3-(dimethylamino)propyl]-3-ethyl-carboimide methiodide. The antiangiogenic activity of RNASET2 $(0.5-10 \mu \mathrm{g} / \mathrm{ml})$ was assessed by human umbilical vein endothelial (HUVE) cells assay in the presence of $1 \mu \mathrm{g} / \mathrm{ml}$ angiogenin, basic fibroblast growth factor (bFGF) or recombinant human vascular endothelial growth factor (VEGF). Cell-colony formation was examined in human colon HT29 cancer cells to assess the anticarcinogenic activity of RNaseT2 or the enzymatic inactivated (EI)-RNaseT2 $(1 \mu \mathrm{g} / \mathrm{ml}$ each). In an athymic mouse xenograft model, LS174T human cancer cells were injected subcutaneously. When tumors were palpable, the mice were treated for 3 weeks with RNASET2 $(1 \mathrm{mg} / \mathrm{kg}$ ), paclitaxel (10 or $15 \mathrm{mg} / \mathrm{kg}$ ) or a combination of the two drugs. The recombinant RNASET2 was found to be a $27-\mathrm{kDa}$ glycoprotein possessing RNase activity and actin-binding ability. In HUVE cells, RNASET2 inhibited angiogenin-, bFGF-, and VEGF-induced tube formation in a dose-dependent manner. RNASET2 significantly inhibited clonogenicity in HT29 cells. EI-RNASET2 showed actin-binding and anticlonogenic effects similar to those of RNASET2, indicating that RNase activity is not a prerequisite for its anticarcinogenic activity. In athymic mice, RNASET2 significantly inhibited the development of LS174T-derived xenografts. A synergistic effect was obtained between RNASET2 and paclitaxel. These results suggest that RNASET2 represents a new class of anticarcinogenic, antiangiogenic drugs and emphasize the advantage of concomitant use of RNASET2 in combined therapy.

\section{PD 12-1}

\section{Antivascular therapy for orthotopic human colon cancer through blockade of the vascular endothelial growth factor and epidermal growth factor receptors}

Takamitsu Sasaki ${ }^{1,2}$, Yasuhiko Kitadai ${ }^{2,3}$, Toru Nakamura $^{2}$, Toshio Kuwai ${ }^{2}$, Langley R. Robert ${ }^{2}$, Isaiah J. Fidler ${ }^{2}$

${ }^{1}$ First Department of Surgery, Fukuoka University School of Medicine, Fukuoka, Japan, ${ }^{2}$ Departments of Cancer Biology, The University of Texas M. D. Anderson Cancer Center, Houston, TX, USA, ${ }^{3}$ Department of Medicine and Molecular Science, Hiroshima University, Japan

Recent data generated from our laboratory suggest that tumors residing in the colon may convey information to their supporting vascular networks by activating the epidermal growth factor receptor (EGF$\mathrm{R})$ and vascular endothelial cell growth factor receptor (VEGF-R) on tumor-associated endothelial cells. To determine whether this paracrine signaling network is critical for tumor progression in the colon, we implanted SW620CE2 human colon cancer cells into the cecal wall. SW620CE2 cancer cells express significant levels of vascular endothelial cell growth factor and transforming growth factor-a (TGF-a), a primary ligand for EGF-R, but lack receptors for both of these proteins. We conducted a rigorous immunohistochemical examination of SW620CE2 tumors 5 weeks after tumor cell implantation and found that tumorassociated endothelial cells expressed both EGFR and VEGFR-2 and that, moreover, both of these tyrosine kinase receptors were activated. Similar to our in vitro observations, no EGF-R or VEGFR-2 was detected on tumor cells in the cecum. To determine whether pharmacologic suppression of EGF-R and VEGFR-2 on tumor-associated endothelial cells could produce therapy of tumors, we treated mice for 5 weeks with AEE788, a dual-inhibitor of EGF-R and VEGF-R, as 
a single agent or in combination with CPT 11 . AEE788 alone or in combination with CPT-11 quenched the phosphorylation of EGF-R and VEGFR-2 on tumor blood vessels. Indeed, combination therapy with AEE788 and CPT-11 signaled for apoptosis of tumor endothelial cells resulting in a significant reduction in tumor microvascular density and tumor cell proliferation and increased cell death in the tumor compartment. Collectively, our results suggest that targeting EGF-R and VEGFR-2 signaling cascades in the vasculature of colon tumors provides a new approach for treatment of colon cancer.

\section{PD 12-2}

TSU-68, a vascular endothelial growth factor receptor tyrosine kinase inhibitor, suppressed the production of experimental brain metastasis by $3 L L$ cells

Tamir Dolginsren ${ }^{1}$, Seiji Yano ${ }^{1}$, Hirokazu Ogino ${ }^{1}$, Hideki Tomimoto $^{1}$, Soji Kakiuchi ${ }^{1}$, Hisatsugu Goto ${ }^{1}$, Hisanori Uehara ${ }^{1}$, Saburo Sone ${ }^{1}$

${ }^{1}$ Department of Internal Medicine and Molecular Therapeutics, and Department of Molecular and Environmental Pathology, University of Tokushima School of Medicine, Tokushima, Japan

Background Brain metastasis is an important cause of cancer morbidity and mortality. We recently reported that vascular endothelial growth factor (VEGF) facilitates brain metastasis by inducing non-sprouting angiogenesis, suggesting that VEGF is an ideal therapeutic target of brain metastasis. In the present study, we examined the therapeutic efficacy of VEGF receptor tyrosine kinase inhibitor, TSU-68, against experimental brain metastasis. Methods A murine lung cancer cell line, 3LL, and a human dermal microvessel endothelial cell line, HMVEC, were used in the study. The effect of TSU68 on proliferation of $3 \mathrm{LL}$ and HMVEC in vitro was determined by MTT assay. For experimental brain metastasis model, $3 \mathrm{LL}$ cells were inoculated into the internal carotid artery of C57/BL-6 mice, and the mice were given oral treatment with TSU-68 $(800 \mathrm{mg} / \mathrm{kg})$ on days $7-20$. The mice were killed on day 21 . The brain was sliced in $2 \mathrm{~mm}$-thickness, and the brain metastasis was evaluated. Proliferating cells (BrdU) and vascularization (CD31) in the brain metastasis were evaluated by immunohistochemistry. Results TSU-68 inhibited VEGF-induced proliferation of HMVEC in a dosedependent manner, while it had no effect on the proliferation of $3 \mathrm{LL}$ cells in vitro. $3 \mathrm{LL}$ cells inoculated into the carotid artery produced metastatic colonies $(3-5 \mathrm{~mm}$ in diameter) into the ventricule and parenchyma of the brain by day 21 . Treatment with TSU-68 markedly inhibited the production of the brain metastasis, in terms of the incidence and diameter of the metastatic colonies. Histological analyses revealed that treatment with TSU-68 reduced the number of enlarged tumor-associated vessels and proliferating tumor cells. Conclusions The VEGF receptor tyrosine kinase inhibitor, TSU-68, inhibited the production of brain metastasis by suppressing the formation of enlarged tumor vessels. Therefore, TSU-68 may be a useful molecular targeted drug against lung cancer brain metastasis.

\section{PD 12-3}

\section{Antitumor activity of ZD6474, a potent inhibitor of vascular endothelial growth factor signaling and epidermal growth factor, in the metastatic orthotopic brain tumor model}

Doo-Sik Kong, Ji-Won Jeon, Hyun jung Jeon, Do-Hyun Nam

Department of Neurosurgery, Samsung Biomedical Research Institute Samsung Medical Center, Sungkyunkwan University School of Medicine, Seoul, Korea

Background Vascular endothelial growth factor (VEGF) and epidermal growth factor (EGF) have been strongly implicated in the metastatic spread of tumor. The purpose of this study was to examine the antitumor effect of ZD6474, an orally available inhibitor of VEGF receptor-2 (VEGFR-2) and EGF receptor (EGFR) tyrosine kinases, on the tumor growth in the available orthotopic brain metastasis models and to compare the results of two inoculation methods (direct intracranial (IC) inoculation and internal carotid artery (ICA) injection model). Methods The human breast carcinoma cells with high metastatic potentiality (MDA-MB435LVBR) were injected with the direct intracranial (IC) inoculation $\left(5 \times 10^{5}\right.$ cells $\left./ 100 \mu \mathrm{l}\right)$ and internal carotid artery (ICA) injection $\left(5 \times 10^{4}\right.$ cells $\left./ 100 \mu \mathrm{l}\right)$ in the Balb/ c-nu female mice. Daily oral treatment with ZD 6474 $(50 \mathrm{mg} / \mathrm{kg}$ ) was initiated on day 14 . Mice were sacrificed on day 28. The frequency of tumor occurrence and the mass size were measured and compared between two different inoculation methods (IC and ICA inoculation models). Results There was a significant reduction of tumor volume in the daily ZD6474 treatment group compared with the control group in both IC and ICA models. Treatment with ZD6474 led to marked inhibition of metastatic tumor growth (median size: $3.5 \mathrm{~mm}^{3}$, 1.6-13.9 $\left.\mathrm{mm}^{3}\right)$, as compared with the control group (median size: $62.4 \mathrm{~mm}^{3}, 11.5-206.9 \mathrm{~mm}^{3}$ ) in the IC inoculation model. Treatment with ZD6474 led to also 
marked reduction of tumor volume (median size: $7.5 \mathrm{~mm}^{3}, 5.4-22.4 \mathrm{~mm}^{3}$ ), as compared with the control group (median size: $61.4 \mathrm{~mm}^{3}, 25.7250 .8 \mathrm{~mm}^{3}$ ) in the ICA injection model. In the ICA injection model, all five control mice had large intracranial metastasis but only 3 out of 6 mice that were treated with ZD6474 had very small masses. Although there was also a significant reduction of tumor volume in ZD6474-treated mice, every mouse in the IC inoculation model had masses regardless to the treatment. Conclusions These results suggest that simultaneous inhibition of both EGFR and VEGFR2 signaling pathway has an effective therapeutic value in inhibiting the growth of metastatic brain tumor and that the direct IC inoculation as well as ICA injection method has a similar result in terms of reduction of tumor growth. However, the tumor uptake or incidence according to the treatment may be evaluated only in the ICA injection model. Therefore, for the purpose of the translational research for the brain metastasis, we can use either two orthotopic models simultaneously or ICA injection model only.

\section{PD 12-4}

\section{Simultaneous inhibition of EGFR, VEGFR, and PDGFR phosphorylation in combination with gemcitabine produces therapy of human pancreatic carcinoma and prolongs survival in an orthotopic nude mouse model}

Kenji Yokoi ${ }^{1}$, Takamitsu Sasaki ${ }^{2}$, Corazon D. Bucana ${ }^{2}$, Dominic Fan ${ }^{2}$, Yasuhiko Kitadai ${ }^{2}$, Toshio Kuwai ${ }^{2}$, Isaiah J. Fidler ${ }^{2}$

${ }^{1}$ Department General and Cardiothoracic Surgery, Kanazawa University, Kanazawa, Japan, ${ }^{2}$ Department of Cancer Biology, University of Texas MD Anderson Cancer Center, USA

Although gemcitabine has been accepted as the first-line chemotherapeutic reagent for advanced pancreatic cancer, its response rate and survival is not sufficient. Because epidermal growth factor receptor (EGFR), vascular endothelial growth factor receptor (VEGFR), and platelet-derived growth factor receptor (PDGFR) modulate tumor progression, we hypothesized that inhibition of phosphorylation of all three on tumor cells, tumor-associated endothelial cells, and stroma cells would improve the efficacy of gemcitabine and prolong survival in an orthotopic pancreatic tumor model in nude mice. L3.6pl, a human pancreatic cancer cell growing in the pancreas, and tumor-associated endothelial cells in this model highly expressed phosphorylated EGFR, VEGFR, and PDGFR. Oral administration of AEE788 (dual tyrosine kinase inhibitor against EGFR and VEGFR) decreased phosphorylation of EGFR and VEGFR. PDGFR phosphorylation was inhibited by STI571. Although i.p. injection of gemcitabine showed limited inhibitory effect on tumor growth, its combination with AEE788 and STI571 produced more than $80 \%$ inhibition of tumor growth and prolonged survival in parallel with increases in number of tumor cells and tumor-associated endothelial cell apoptosis, decreased microvascular density, and decreased proliferation rate. STI571 treatment also decreased pericyte coverage on tumor-associated endothelial cells. Thus, inhibiting phosphorylation of EGFR, VEGFR, and PDGFR in combination with gemcitabine enhanced the efficacy of gemcitabine, resulting in inhibition of experimental human pancreatic cancer growth and significant prolongation of survival.

\section{PD 12-5}

Therapeutic efficacy of anti-vascular endothelial growth factor antibody, bevacizumab, against orthotopically implanted human pleural mesothelioma cells in SCID mice

Qi Li ${ }^{1}$, Tamir Doljinsuren ${ }^{1}$,Seiji Yano ${ }^{1}$, Hirokazu Ogino $^{1}$, Tadaaki Yamada ${ }^{1}$, Hideki Tomimoto ${ }^{1}$, Takafumi Todoroki ${ }^{1}$, Shinsaku Otsuka ${ }^{1}$,SojiKakiuchi ${ }^{1}$, Hisatsugu Goto ${ }^{1}$, Hisanori Uehara ${ }^{2}$, Saburo Sone ${ }^{1}$

${ }^{1}$ Department of Internal Medicine and Molecular Therapeutics, University of Tokushima School of Medicine, Tokushima, Japan, ${ }^{2}$ Department of Molecular and Environmental Pathology, University of Tokushima School of Medicine, Tokushima, Japan

Background Malignant pleural mesothelioma (MPM) grows aggressively with dissemination in the thoracic cavity and frequently produces malignant pleural effusion. MPM is refractory to conventional chemotherapy and radiotherapy, and it also has a poor prognosis (median survival time from onset is approximately 1 year). Therefore, novel effective therapies are necessary for improving the prognosis of this disease. Since vascular endothelial growth factor (VEGF) is reported to be an ideal therapeutic target, we examined the therapeutic efficacy of anti-human VEGF neutralizing antibody, bevacizumab, against orthotopically inoculated human MPM cells. Methods Human MPM cell lines, EHMES-10 (VEGF high producing) and MSTO211H (VEGF-low producing) and a human endothelial cell line, HMVEC), were used in the study. The effect of bevacizumab on proliferation of MPM and HMVEC in vitro was determined by MTT assay. For orthotopic 
implantation model, MPM cells $\left(1 \times 10^{6}\right)$ were inoculated into the thoracic cavity of SCID mice. The mice were treated with control IgG or bevacizumab, and production of thoracic tumor and pleural effusion were evaluated. Proliferating cells (BrdU) and vascularization $(\mathrm{CD} 31)$ in the thoracic tumors were evaluated by immunohistochemistry. Results EHMES-10 cells secreted a high level of VEGF, while MSTO-211H cells secreted only a low level. Neither EHMES-10 nor MSTO-211H cells expressed VEGF receptors at protein levels. Bevacizumab inhibited VEGF-induced proliferation of HMVEC in a dose-dependent manner, while it had no effect on the proliferation of MPM cells in vitro. Orthotopically inoculated EHMES-10 produced thoracic tumors and a large volume of bloody pleural effusion by day 28 , while MSTO-211H cells produced thoracic tumors and a small volume of bloody effusions by day 21 . While treatment with bevacizumab (twice a week started on day 7) slightly inhibited the production of thoracic tumors of EHMES-10 and MSTO-211H cells, it dramatically prevented the production of pleural effusion by EHMES-10, but not MSTO-211H cells, consistent with expression of VEGF by MPM cells. Histological analyses revealed that treatment with bevacizumab reduced the number of enlarged tumor-associated vessels and proliferating tumor cells. Conclusions Anti-VEGF antibody, becacizumab, may be a promising agent for controlling pleural effusions of VEGFhigh producing MPM cells, and additional modality to regulate the growth of thoracic tumors may be necessary for controlling disease progression of MPM.

\section{PD 12-6}

\section{Suppression of peritoneal dissemination by the blockade of TNF- $\alpha$ as demonstrated using TNF- $\alpha$ knockout mice/bone marrow chimera mice model and human gastric cancer xenograft model}

\section{Hayao Nakanishi ${ }^{1}$, Yuzuru Ikehara ${ }^{2}$, Yoshinari} Mochizuki $^{3}$, Yasuhiro Kodera ${ }^{4}$, Masae Tatematsu ${ }^{1}$

${ }^{1}$ Division of Oncological Pathology, Aichi Cancer Center Research Institute, Nagoya, Japan, ${ }^{2}$ National Institute of Advanced Industrial Science and Technology, Japan, ${ }^{3}$ Aichi Cancer Center Central Hospital, Department of Gastroenterological Surgery, Japan, ${ }^{4}$ Department of Surgery II, Nagoya University School of Medicine, Japan

We previously reported that $\mathrm{TNF} \alpha$ promotes peritoneal dissemination of gastric cancer xenograft in nude mice. The aim of this study is to investigate whether peritoneal dissemination can be suppressed by the blockade of
TNF $\alpha$ using two mouse models such as TNF- $\alpha$ knockout $(\mathrm{KO})$ mice/bone marrow chimera mice model and human gastric cancer xenograft model. We found that mouse Lewis carcinoma cells (P-29) disseminated into the whole peritoneal cavity including abdominal wall peritoneum in wild type C57BL mice after intraperitoneal injection, whereas in the TNF- $\alpha \mathrm{KO}$ mice, tumor cells only metastasized to the omentum and mesentery in a milky spot-dependent manner without dissemination as well as growth stimulation, leading to the significant longer survival of KO mice than wild type mice. This restricted peritoneal metastasis was canceled in chimeric KO mice transplanted with wild type bone marrow, in which TNF- $\alpha$ expressing leukocyte recruited into the peritoneal cavity. Addition of intraperitoneal chemotherapy with paclitaxel inhibited tumor growth in the peritoneal cavity and significantly increased survival time of KO mice rather than wild type mice because of inhibition of both growth and peritoneal dissemination of tumor cells. Furthermore, peritoneal dissemination of human gastric cancers in nude mice was partially inhibited by the treatment with anti human TNF- $\alpha$ antibody (infliximab), resulting in a longer survival of the treated mice. These results indicate that TNF- $\alpha$ derived from host inflammatory leukocytes as well as tumor cells in the peritoneal cavity is responsible for peritoneal dissemination of tumor cells and suggest that anti TNF- $\alpha$ therapy in combination with chemotherapy would be a new potential therapeutic modality for protection of peritoneal dissemination and therefore prevention of peritoneal recurrence after curative surgery in gastric cancer patients.

\section{PD 12-7}

\section{Therapy of multidrug resistant prostate cancer by targeting the epidermal growth factor receptor and vascular endothelial growth factor receptor on tumor-associated endothelial cells}

Joseph E. Busby, Sun Jin Kim, M. Sertac Yazici, Dominic Fan, Isaiah J. Fidler

\section{Houston, TX}

Introduction and Objective Presently there are no available therapeutic options to overcome the androgen independence and chemotherapy-resistance that develop during the treatment of advanced prostate cancer. The progressive growth of all neoplasms is dependent on development and maintenance of vasculature. Since prostate cancer cells and tumor-associated endothelial cells express activated epidermal growth factor receptor (EGFR) and vascular endothelial growth factor receptor 
(VEGFR), we determined whether inhibition of these receptors can overcome multidrug resistant (MDR) human prostate cancer cells in the prostate of nude mice. Methods The PC-3MM2-MDR prostate cancer cell line was selected in vitro and found to have a 76fold increased resistance as compared to the parental line. The MDR cells were implanted into the prostates of nude mice and 14 days later the mice were randomized $(n=10)$ to: control (three times per week oral saline and once per week intraperitoneal (i.p.) injection of distilled water); paclitaxel (once per week i.p. injection of $8 \mathrm{mg} / \mathrm{kg}$ paclitaxel); AEE788 (three times per week oral $50 \mathrm{mg} / \mathrm{kg}$ AEE788); and AEE788 plus paclitaxel. After 10 weeks of treatment, the mice were necropsied. Tumor incidence, tumor weight, and incidence of lymph node metastasis were recorded. Immunohistochemical (IHC) analyses were performed on harvested specimens. Results Treatment with paclitaxel was not therapeutic. Treatment with AEE788 or AEE788 plus paclitaxel significantly reduced tumor incidence, tumor weight, and incidence of lymph node metastasis. The combination of AEE788 and paclitaxel completely eliminated lymph node metastasis. IHC data revealed that treatment with AEE788 and AEE788 plus paclitaxel inhibited phosphorylation of EGFR and VEGFR on tumor cells and tumor-associated endothelial cells, decreased tumor cell proliferation and induced apoptosis of tumor-associated endothelial cells leading to apoptosis of tumor cells. Conclusions Inhibition of EGFR and VEGFR pathways by AEE788 combined with paclitaxel produces apoptosis of tumorassociated endothelial cells leading to apoptosis of cancer cells thereby overcoming chemotherapy-resistance of metastatic MDR prostate cancer cells.

\section{PD 12-8}

\section{VEGFRs inhibitor E7080 inhibits lymph node metastasis of human breast carcinoma, by preventing murine lymphatic endothelial cells from lymphangiogenesis}

Junji Matsui $^{1}$, Akihiko Tsuruoka ${ }^{1}$, Koji Morimoto ${ }^{2}$, Toshimitsu Uenaka ${ }^{1}$, Yuji Yamamoto ${ }^{1}$, Tatsuo Watanabe $^{3}$, Masayuki Okada ${ }^{2}$, Shinya Abe ${ }^{1}$, Toshiaki Wakabayashi $^{1}$, Makoto Asada ${ }^{1}$

${ }^{1}$ Discovery Research Laboratories II, Eisai Co., Ltd., Ibaraki, Japan, ${ }^{2}$ KAN Research Institute, Kyoto, Japan, ${ }^{3}$ New Product Development, Clinical Research Center, Eisai Co., Ltd., Tokyo, Japan

The spread of tumor cells into regional lymph nodes through the lymphatic system correlates with poor prognosis in breast, lung and gastrointestinal tract tumors. Vascular endothelial growth factor (VEGF)-C/DVEGF receptor-3 (VEGFR3) is a key signaling in this process. VEGF-C and D bind to and activate VEGFR3, which is primarily expressed on lymphatic endothelium, and also bind to VEGFR2 (only after those ligands are fully processed). In this study, we constructed a lymph node metastasis model of breast carcinoma and evaluated the potency of E7080 as an anti-metastatic agent through the mechanism of inhibiting lymphangiogenesis. In cell free and cell based kinase assays with IC50 of subnanomolar concentrations, E7080, a multi-kinase inhibitor, potently inhibits VEGFR1-3, particularly VEGFR2 and-3. MDA-MB-231, which is a breast tumor cell line over-expressing both VEGF-A and-C, shows lymphangiogenesis and angiogenesis in tumor tissue and both lymph node and lung metastases. MDA-MB-435, which is a breast tumor cell line expressing only VEGF-A, shows angiogenesis in tumor tissue but not lymphangiogenesis leading to either lung or lymph node metastases. The administration of E7080 at $100 \mathrm{mg} / \mathrm{kg}$ clearly decreased tumor growth of ortho-topically transplanted MDAMB-231, and also inhibited lymph node and lung metastases. On the other hand, the administration of Bevacizumab (Avastin, a recombinant humanized monoclonal antibody directed against VEGF-A which is a ligand for VEGFR1 and VEGFR2) at $800 \mu \mathrm{g} /$ head did not decrease lymph node metastases, despite showing significant anti-tumor effect on the primary tumor and lung metastases. In an immunohistochemical analysis of tumor specimen, E7080 diminished both tumor angiogenesis and lymphangiogenesis, which were measured by CD31 staining and LYVE-1 staining, respectively. In comparison, Bevacizumab diminished only tumor angiogenesis but was ineffective for lymphangiogenesis. We also evaluated the effect of $\mathrm{E} 7080$ against lymph node metastases after surgical resection of primary tumor tissues, which may refer to adjuvant therapy after surgical resection in breast carcinoma patients. E7080 showed anti-tumor regression against lymph node metastases and disappearance of lymph node metastases in $2 / 4$ mice. These results clearly showed that the blockage of all three VEGFRs signaling is a promising therapeutic strategy because VEGFR3 mediated signal plays important roles in tumor lymph nodes metastasis, and E7080 is useful for such purpose.

\section{PD 12-9}

Immunohistochemical visualised inhibition of Src-phosphorylated substrates FAK and Paxillin by the Src/Abl kinase inhibitor AZD0530 demonstrates a dynamic inhibition 
of Src activity in human tumour xenograft tissues following oral dosing

Tim P. Green, Armelle Logie, Vivien Jacobs, Mike Fennell, Judith Hargreaves, Russell Westwood, Nicola Haupt, Robert Wilkinson, Barry Davies, Paul Elvin

AstraZeneca, Alderley Park, Macclesfield, Cheshire, UK

We have reported previously that AZD0530, an oral, dual-specific inhibitor of Src and Abl tyrosine kinases, potently reverses Src driven invasion phenotypes in cancer cells in vitro and can inhibit metastasis from both subcutaneous and orthotopic implantation sites in vivo (Green et al., oral communication, AACR 2005)

Compared with its anti-invasive activity, AZD0530 has a less consistent anti-proliferative profile when screened in in vitro and in vivo models of tumour cell growth. In a panel of human tumour xenografts, once daily AZD0530 treatment $(12.5-50 \mathrm{mg} / \mathrm{kg} /$ day p.o) failed to inhibit primary tumour growth in 5/9 xenograft models screened (HPAC (pancreas); Lovo, HT-29, and Colo205 (colon); and ZR-75.1 (breast)).

In the remainder of the xenograft panel $(n=4$ : Calu-6 (lung); BT-474C and MDA-231 (breast); and AsPC-1 (pancreas)), daily AZD0530 treatment resulted in a moderate, but dose independent growth delay $(\sim 30$ $50 \%)$.

Using an example of an AZD0530 non-responsive xenograft (Lovo) and a responsive xenograft (Calu-6), the phosphorylation of Src TK substrates Paxillin and Focal Adhesion Kinase (FAK) was significantly reduced in both acute and chronic dosing regimes in both the responsive and nonresponsive xenografts. The observed PK/PD relationship provides encouragement for the use of such biomarkers to demonstrate inhibition of Src target mechanism in man and establish PK/PD in AZD0530 clinical studies.

The data also reinforce previous observations that suggest that AZD 0530 selective inhibition of Src and $\mathrm{Abl}$ kinases in tumours is only infrequently associated with a dose responsive inhibition of growth in xenograft models. Together, these data suggest that AZD0530 monotherapy in the clinic may provide clinical benefit through AZD0530 inhibition of tumour invasion mechanisms and that biomarkers such as FAK and Paxillin could provide early evidence of the drug inhibiting an invasion phenotype in man.

The data also suggest that appropriate combination strategies should be explored with AZD0530 to com- plement the anti-invasive activity and maximise the potential clinical benefit of the drug.

AZD0530 is currently in early phase clinical development.

\section{PD 12-10}

\section{RhoGDI $\beta$ lacking the $\mathbf{N}$-terminal regulatory domain suppresses metastasis by promoting anoikis in $\mathrm{v}$-src transformed cells}

Takahide Ota ${ }^{1}$, Masayo Maeda ${ }^{2}$, Manabu Murakami ${ }^{1}$, Tsutomu Takegami ${ }^{1}$, Akifumi Kanda ${ }^{3}$, Masaaki Tatsuka $^{3}$

${ }^{1}$ Department of Molecular Oncology and Virology, Medical Research Institute, Kanazawa Medical University, Ishikawa, Japan, ${ }^{2}$ Department of Chemistry, Kanazawa Medical University, Ishikawa, Japan, ${ }^{3}$ Department of Regulatory Radiobiology, Research Institute for Radiation Biology and Medicine, Hiroshima University, Hiroshima, Japan

Rho guanine nucleotide dissociation inhibitors (RhoGDIs) regulate the activity of Rho family GTPases. RhoGDI $\beta$ (LyGDI/GDID4/RhoGDI2) has two caspase cleavage sites after Asp19 and Asp55. The resulting cleavage products, $\Delta \mathrm{N}(1-19)$ RhoGDI $\beta$ and $\Delta \mathrm{N}(1-55)$ RhoGDI $\beta$, are expressed in cells under conditions that activate caspases. $\Delta \mathrm{N}(1-19)$ RhoGDI $\beta$, which can inhibit GDP dissociation, is implicated in the process of apoptosis, whereas the physiological roles for $\Delta \mathrm{N}$ (1-55) RhoGDI $\beta$, which lacks the ability to inhibit GDP dissociation, are largely unknown. To explore the roles of $\Delta \mathrm{N}$ (1-55) RhoGDI $\beta$, we examined the phenotypes of $v$-src transformed metastatic fibroblasts transfected with plasmids for expressing $\Delta \mathrm{N}$ (1-55) RhoGDI $\beta$. Although the expression of $\Delta \mathrm{N}$ (1-55) RhoGDI $\beta$ had no effect on the rate of growth in vitro, it suppressed experimental metastasis and decreased the rate of growth in vivo. In addition, $\Delta \mathrm{N}$ (1-55) RhoGDI $\beta$-expressing cells had enhanced adhesion to fibronectin, laminin, and collagens but reduced retention in the lung after intravenous injection. Also, the expression of $\Delta \mathrm{N}$ (1-55) RhoGDI $\beta$ promoted anoikis without affecting the levels of activated Rac 1 or Cdc 42. Thus, $\Delta \mathrm{N}$ (1-55) RhoGDI $\beta$ appears to suppress metastasis by promoting anoikisin $v$-src transformed fibroblasts. These results suggest that proteolytically produced $\Delta \mathrm{N}(1-55)$ RhoGDI $\beta$ participates in anoikis signaling by mechanisms other than inhibiting Rho family GTPases. 